\title{
NEUROBIOLOGICALLY-BASED CONTROL SYSTEM FOR AN ADAPTIVELY WALKING HEXAPOD
}

\author{
by \\ WILLIAM ANTHONY LEWINGER
}

Submitted in partial fulfillment of the requirements

For the degree of Doctor of Philosophy

Research Advisor: Dr. Roger D. Quinn

Department of Electrical Engineering and Computer Science

Systems and Control Engineering

CASE WESTERN RESERVE UNIVERSITY

May 2011 


\section{CASE WESTERN RESERVE UNIVERSITY \\ SCHOOL OF GRADUATE STUDIES}

We hereby approve the thesis/dissertation of

William Lewinger

candidate for the

Doctor of Philosophy

degree *.

\begin{tabular}{l} 
(signed) $\quad$ Roger Quinn (EMAE/EECS) \\
(Chair of the Committee) \\
Michael Branicky (EECS) \\
\hline
\end{tabular}

Roy Ritzmann (BIOL)

Wyatt Newman (EECS)

(Date) $\quad 04$ January 2011

* We also certify that written approval has been obtained for any proprietary permission contained therein. 
Copyright C 2011 by William Anthony Lewinger All rights reserved 
This dissertation is dedicated to Roger, who has been a constant supporter, advocate, mentor, advisor, and friend throughout the whole graduate studies process.

Thanks Roger!
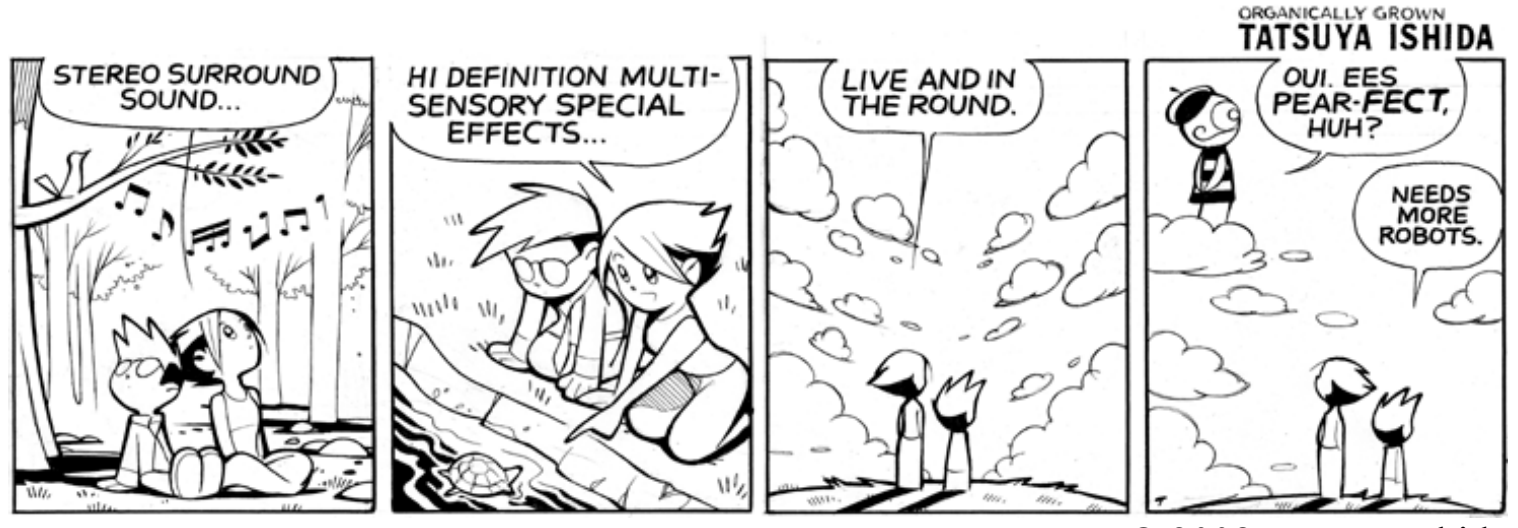

(C) 2008 Tatsuya Ishida 


\section{TABLE OF CONTENTS}

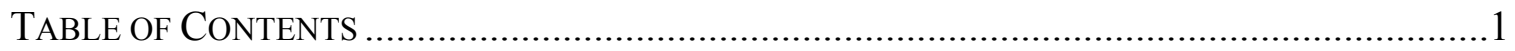

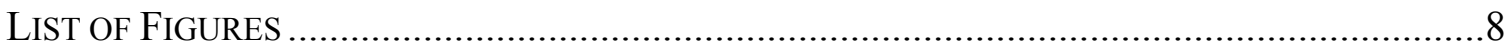

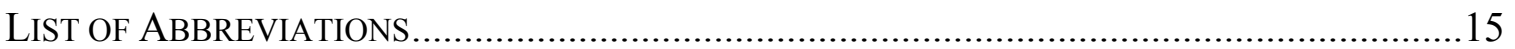

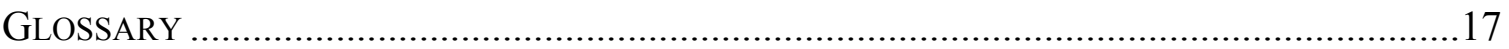

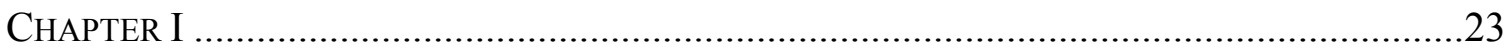

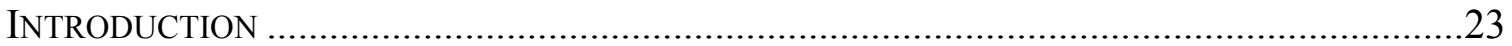

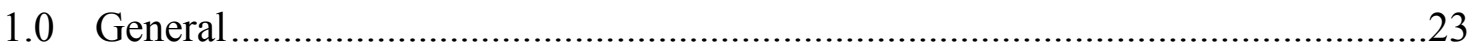

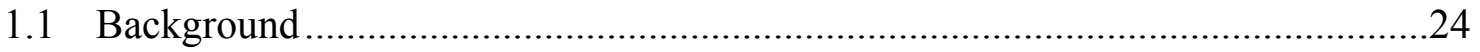

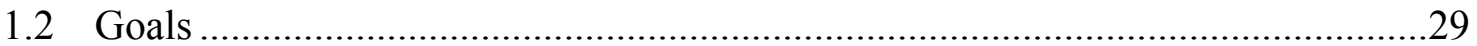

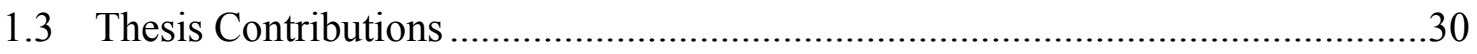

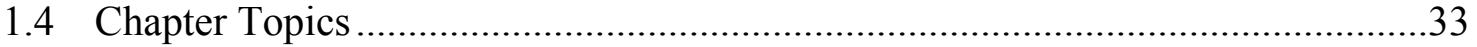

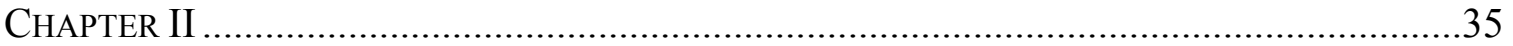

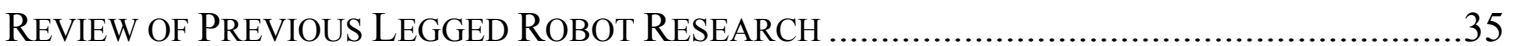

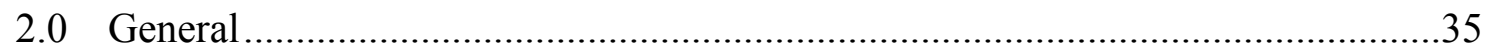

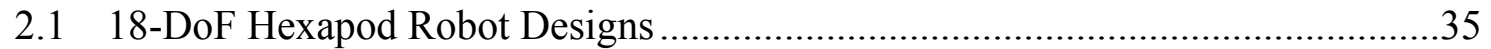

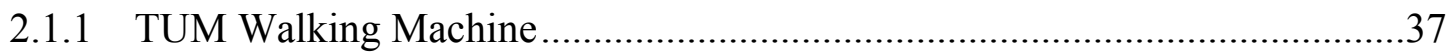

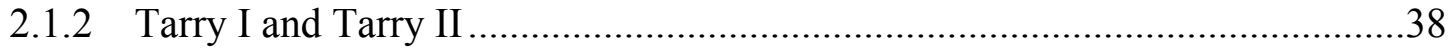

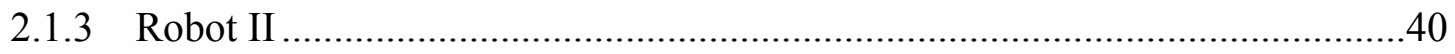

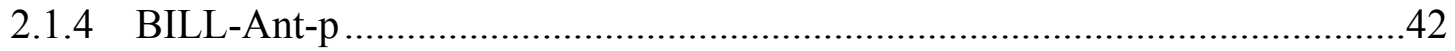

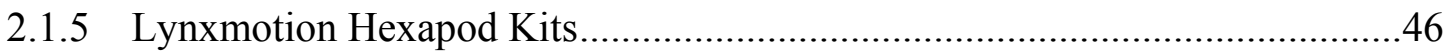


2.1.6 AMOS-WD06.

2.1.7 Micromagic Systems Hexapod Robot Series..................................................51

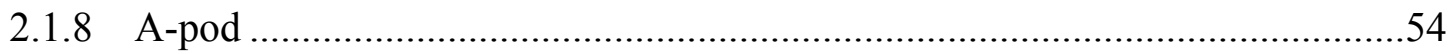

2.1.9 Additional 18-DoF Hexapod Robots ……………...........................................57

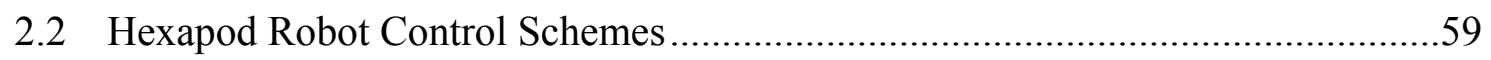

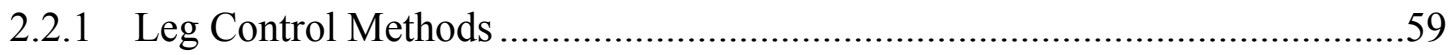

2.2.1.1 Pure Mechanical Systems ………………............................................59

2.2.1.2 Software-Implemented Fixed Step Path .................................................61

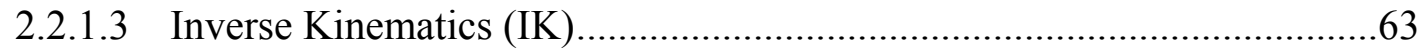

2.2.1.4 Central Pattern Generators (CPGs) and Artificial Neural Networks

(ANNs)

2.2.2 Leg Coordination Methods ...........................................................................68

2.2.2.1 Coupled Mechanical Systems …………………......................................70

2.2.2.2 Fixed Programmed Gaits ......................................................................72

2.2.2.3 Dynamically Selectable Gaits .................................................................73

2.2.2.4 Oscillators / Central Pattern Generators (CPGs) ........................................74

2.2.2.5 Cruse's Rules for Leg Coordination .....................................................77

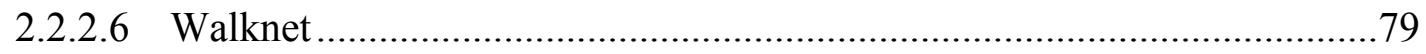

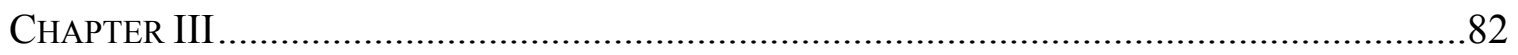

BIOLOGICALLY-INSPIRED LEGGED LOCOMOTION - ANT - AUTONOMOUS (BILL-ANT-A)

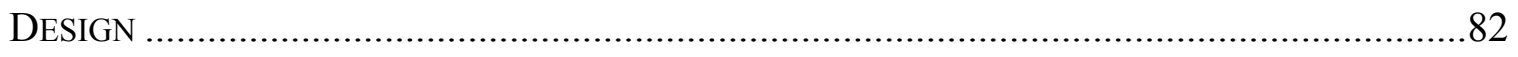

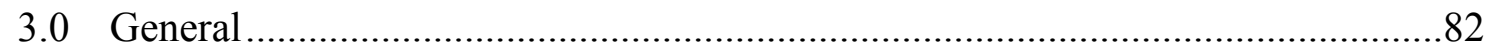




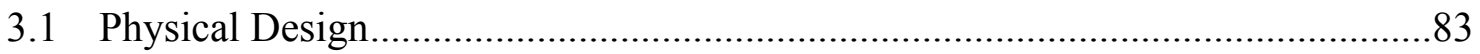

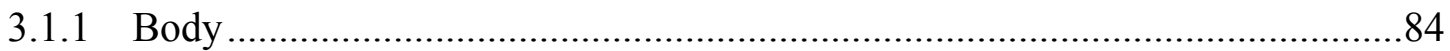

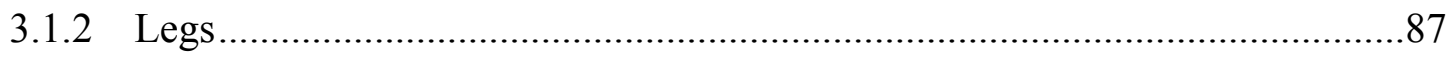

3.1.3 Neck and Light Sensors ...........................................................................93

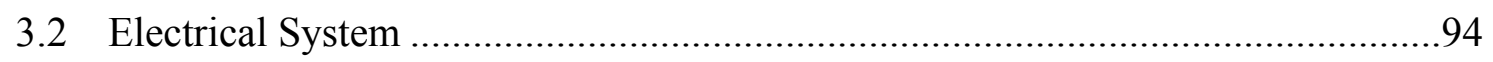

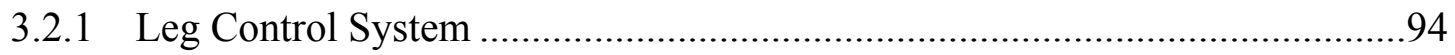

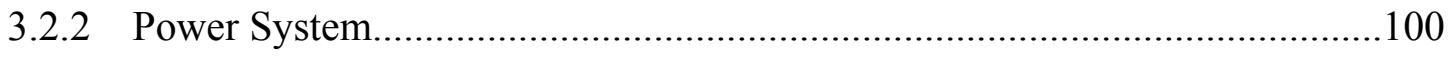

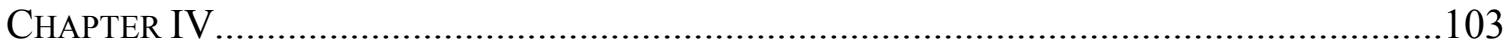

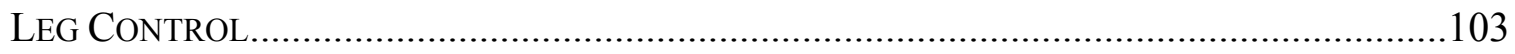

SENSORY-COUPLED ACTION SWITCHING ModULES (SCASM) ........................................103

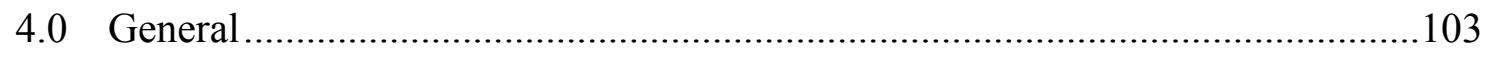

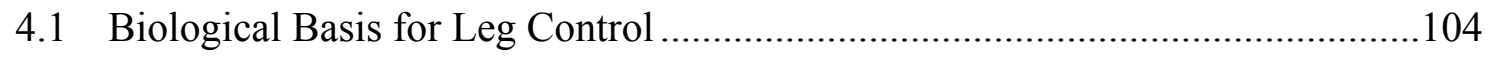

4.2 Introduction to Sensory-Coupled Action Switching Modules (SCASM) ............106

4.3 Implementation of SCASM for Intra-Leg Joint Control.......................................112

4.3.1 Single-Leg Test Platform.......................................................................112

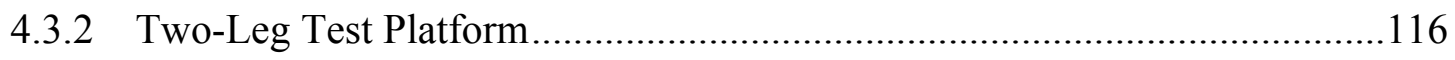

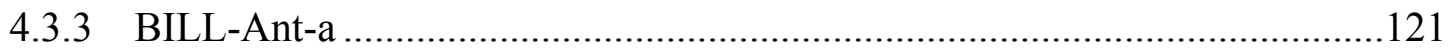

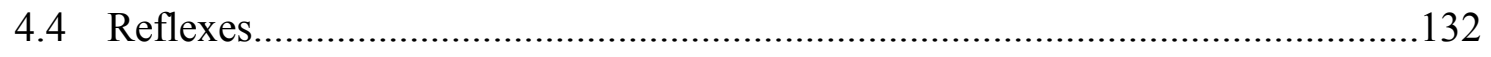

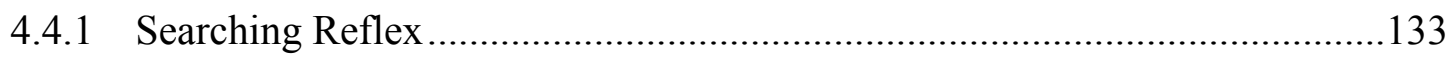

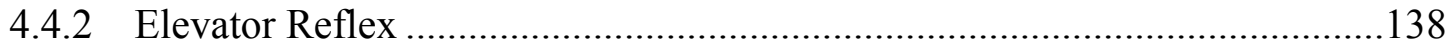

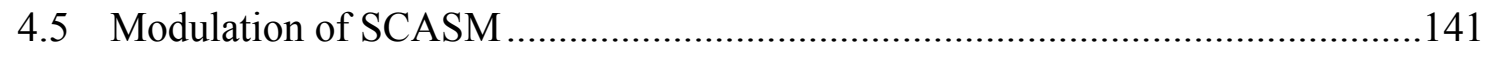


CHAPTER V.

LEG COORDINATION

BIOLOGICALLY-INSPIRED LEGGED LOCOMOTION - LOW COMPUTATION POWER EMERGENT

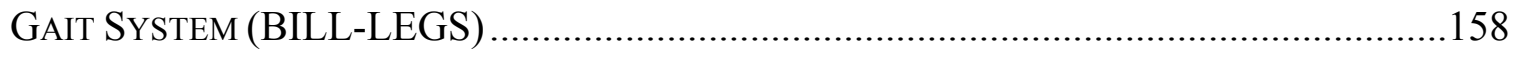

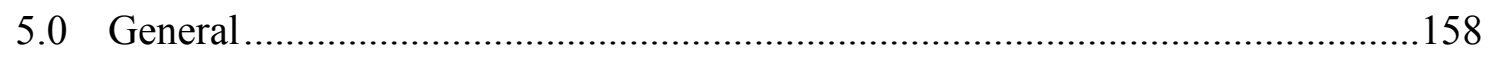

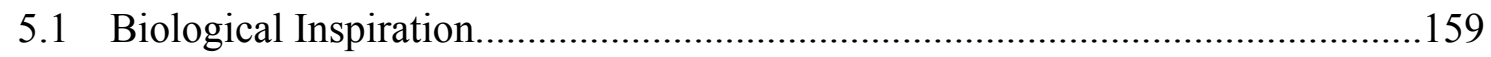

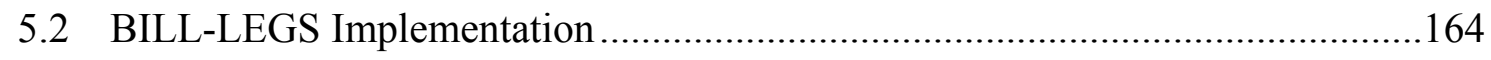

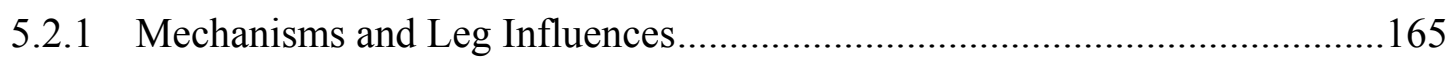

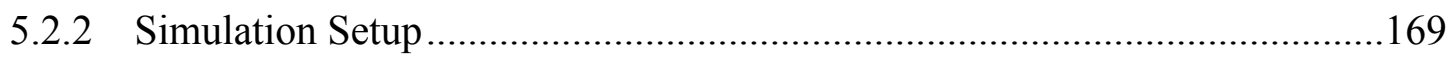

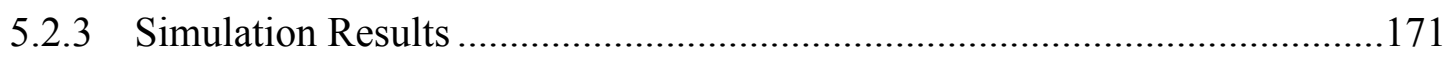

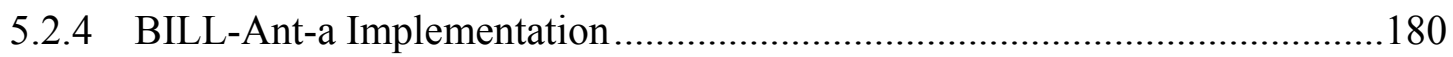

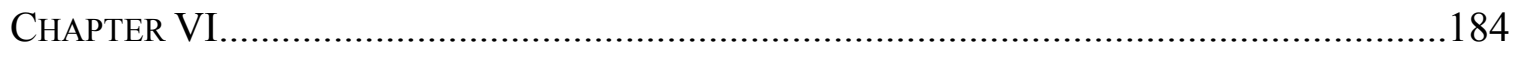

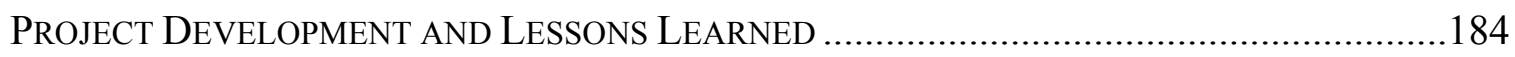

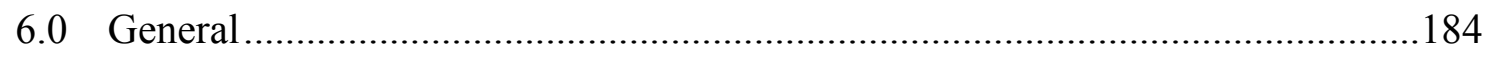

6.1 Two-Leg Test Platform: BILL-Ant-p2 …………….....................................184

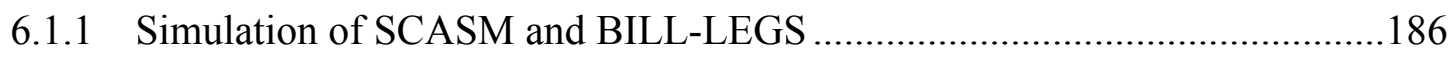

6.1.2 BILL-Ant-p2 with SCASM, BILL-LEGS, Reflexes, and Goal Seeking.......187

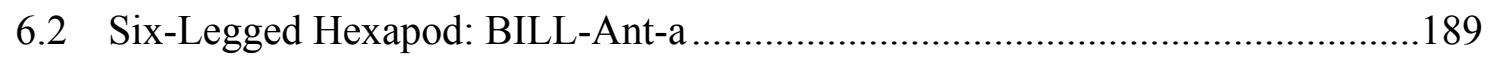

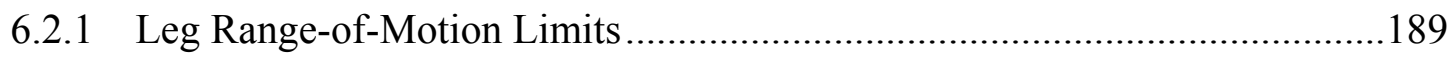

6.2.2 Replacement of the Right-Rear FSR Sensor.................................................190

6.2.3 Alteration of Middle Leg Gait Controllers Creates CPG-Like Behavior ......191

6.2.4 Update of CTr Joint "Muscle Model” to Promote Posture Control................192 
6.2.5 Goal Searching Behavior for When a Goal Is Not In View 193

6.2.6 Update to BILL-Ant-a Goal-Seeking-Based Speed Control

CHAPTER VII 195

SyStem PERFoRMANCE. 195

7.0 General 195

7.1 BILL-Ant-a System, Subsystem, and Component Masses 195

7.2 BILL-Ant-a Motor Current Consumption .196

7.3 BILL-Ant-p2 on Flat Terrain 197

7.3.1 BILL-Ant-p2 Basic Walking 197

7.3.2 BILL-Ant-p2 Reflex Performance on Cluttered Terrain .201

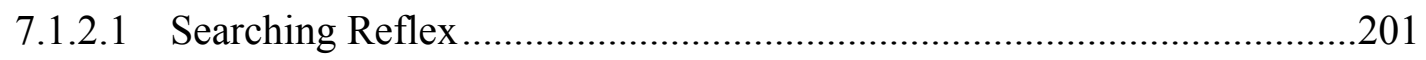

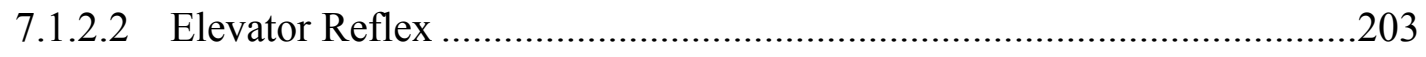

7.3.3 BILL-Ant-p2 Modulated Walking with Goal Seeking …………..................204

7.4 BILL-Ant-a on Flat and Limited Height Variation Terrain................................204

7.4.1 BILL-Ant-a Basic Walking.....................................................................205

7.4.2 BILL-Ant-a Reflex Performance on Flat Terrain with Obstacles .................206

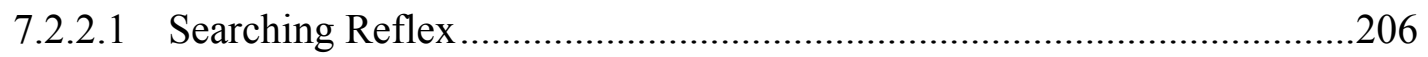

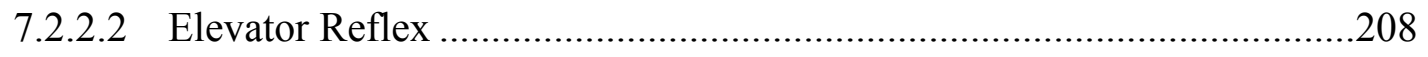

7.4.3 BILL-Ant-a Modulated Walking and Goal Seeking.....................................211

7.5 BILL-Ant-a in the Random Elevation Field Test Arena ....................................212

7.5.1 Irregular Terrain Navigation (No Goal Seeking)........................................213

7.5.2 Irregular Terrain Navigation with Goal Seeking ..........................................215 


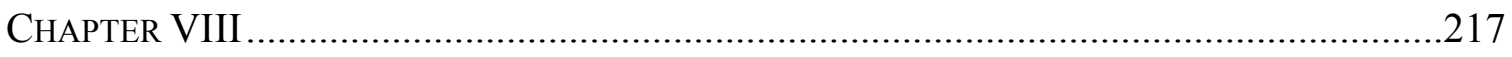

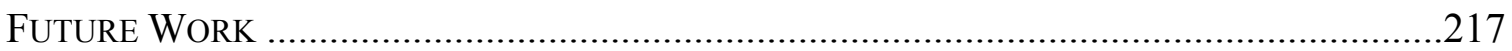

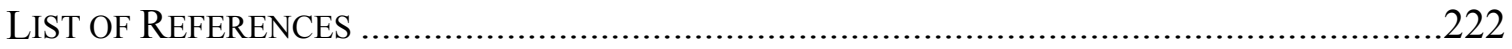




\section{LIST OF TABLES}

Table 2.1. Micromagic Systems hexapod robot specifications......................................53

Table 2.2. 3-DoF per leg hexapod robot general information chart. ............................57

Table 2.3. Selected 3-DoF per leg hexapod robot specifications comparison chart.........58

Table 4.1. SCASM state transition threshold values. ................................................111

Table 4.2. "Muscle activation" values for the Two-Leg Test Platform.........................120

Table 4.3. "Muscle activation" values for BILL-Ant-a............................................129

Table 4.4. Simulator SCASM value equations for foot path headings of $\leq 0 \mathrm{deg}$ (stepping to the right)

Table 4.5. Simulator SCASM value equations for foot path headings of $>0 \mathrm{deg}$

(stepping to the left)

Table 4.6. BILL-Ant-a SCASM value equations for straight walking and right turn

foot path headings

Table 4.7. BILL-Ant-a SCASM value equations for left turn foot path headings 155

Table 7.1. BILL-Ant-a component and subsystem masses........................................196

Table 7.2. BILL-Ant-a motor current consumption for various tasks. 197 


\section{LIST OF FIGURES}

Figure 1.1. The Biologically-Inspired Legged Locomotion Ant - autonomous (BILLAnt-a) robot.

Figure 2.1. TUM Walking Machine .37

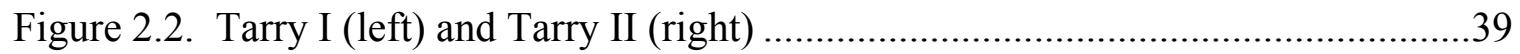

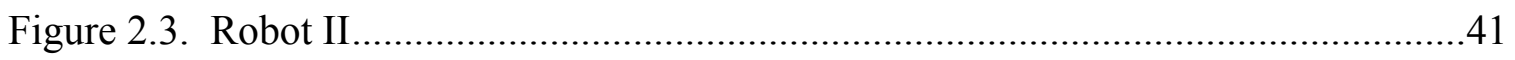

Figure 2.4. The Biologically-Inspired Legged Locomotion - Ant - prototype (BILL-Ant-p) robot

Figure 2.5. BILL-Ant-p user interface with Drive Control window shown. .45

Figure 2.6. Collection of Lynxmotion, Inc. hexapod robots:

Figure 2.7. AMOS-WD06 is a hexapod robot designed by Poramate Manoonpong .......50

Figure 2.8. Collection of Micromagic Systems hexapod robots: .52

Figure 2.9. A-pod hexapod designed by Kåre Halvorsen (a.k.a. Zenta). .54

Figure 2.10. MechaRoach II (left), developed by Terence Wei, uses a four-bar mechanism (right) to mechanically determine the foot path

Figure 2.11. Lynxmotion, Inc. Visual Sequencer software. 62

Figure 2.12. Six-pose stepping pattern option for the BlackWido hexapod.

Figure 2.13. Parabolic swing and straight-line line stance foot trajectories for BILL-Ant-p stepping motion. .65

Figure 2.14. Leg movement central pattern generator for AMOS-WD06.....................67

Figure 2.15. Gait patterns progressing from slow to fast walking. .69 
Figure 2.16. Wind-up toy hexapod (MxyKikker) with mechanically coupled legs (via gears) that walks in an alternating tripod ........................................... 70

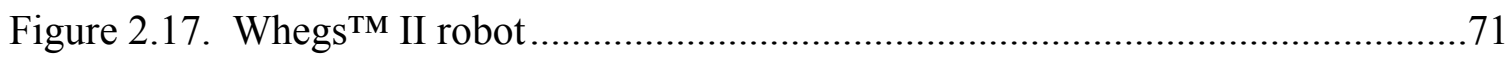

Figure 2.18. Leg control and coordination network for Robot I................................76

Figure 2.19. Cruse's basic rule set for stick insect walking....................................78

Figure 2.20 Foot path and signal forms for Mechanisms 1, 2, and 3 with respect to leg position as implemented on BILL-Ant-p ........................................... 79

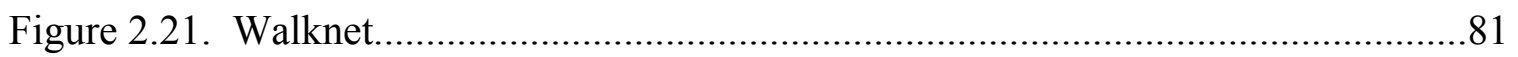

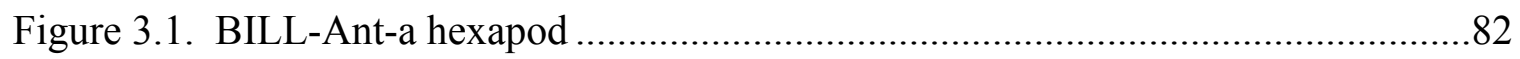

Figure 3.2. Acromyrmex versicolor (left, Leafcutter ant found in Arizona, USA) and BILL-Ant-p (right) body parts......................................................... 83

Figure 3.3. Robot body section without legs, electronics, or batteries......................... 84

Figure 3.4. Top-view body layout comparison of Pheidole fervida (left, found in Japan) and the BILL-Ant-p robot (right). ................................................... 86

Figure 3.5. Left-front leg of BILL-Ant-a attached to the body with labels....................87

Figure 3.6. Machined coxae with thorax-coxa joint axles installed. .............................89

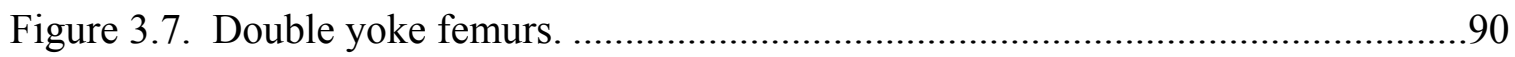

Figure 3.8. BILL-Ant-a foot construction with force-sensitive resistors (left) and the Resistance-Force graph for the FSR elements (right)...............................91

Figure 3.9. The state change diagram shows values from the ADC that allows the leg to determine the condition of contact with the substrate. .92

Figure 3.10. Foot-mounted obstacle detection switch .92 
Figure 3.11. BILL-Ant-p head and BILL-Ant-a neck

Figure 3.12. Microcontroller Network Schematic and Physical Layouts .95

Figure 3.13. Acroname BrainStem GP 1.0 (left) and GP 2.0 (right) microcontrollers.....96

Figure 3.14. BILL-Ant-a electronic and control system connectivity diagram. .99

Figure 3.15. BrainStem microcontroller network for BILL-Ant-a. .100

Figure 4.1. Original Sensory-Coupled Action Switching Modules (SCASM) system connectivity diagram. 108

Figure 4.2. Foot path and joint states for a typical step cycle. .110

Figure 4.3. Single-Leg Test Platform designed by Brandon Rutter and Brian Taylor...113

Figure 4.4. Two-Leg Test Platform.

Figure 4.5. Simple "muscle models" used for the Two-Leg Test Platform.

Figure 4.6. Flow diagrams of the three programs running on each BrainStem leg controller .121

Figure 4.7. ThC joint state diagram. 124

Figure 4.8. CTr joint state diagram. .125

Figure 4.9. ThC joint state diagram. .126

Figure 4.10. Simple "muscle models" used for BILL-Ant-a. 128

Figure 4.11. BILL-Ant-a graphical user interface (GUI). 132

Figure 4.12. Original SCASM diagram (top) and the updated SCASM diagram with the addition of two new biologically-plausible neural pathways (bottom). .134

Figure 4.13. Stepping motions for normal walking and the searching and elevator reflex behaviors. 135 
Figure 4.14. Close-up view of the searching reflex joint state conditions.....................136

Figure 4.15 Close-up view of the elevator reflex joint state conditions. .......................139

Figure 4.16. Cockroach foot paths during turning..................................................143

Figure 4.17. Simulated right leg foot path along the sagittal plane............................144

Figure 4.18. Simulated right leg foot path at $-45 \mathrm{deg}$ to the sagittal plane.....................146

Figure 4.19. Simulated right leg foot path at $+45 \mathrm{deg}$ to the sagittal plane....................147

Figure 4.20. Early neck and stereo phototransistor assembly..................................151

Figure 4.21. Cockroach foot paths during free turning...........................................153

Figure 4.22. Updated phototransistor mounting bracket. ......................................157

Figure 5.1. Mechanism connectivity network for Cruse's method of inter-leg coordination

Figure 5.2. Immunity of leg coordination mechanism weights to variation in the generation of stable gaits for the Cruse method in Robot II.

Figure 5.3. Immunity of leg coordination mechanism weights to variation in the generation of stable gaits for the Cruse method in BILL-Ant-p.....

Figure 5.4. The effects of varying individual starting leg positions (top) and leg movement speeds of individual legs and leg pairs (bottom) on stable gait generation for the Cruse method in BILL-Ant-p.

Figure 5.5. BILL-LEGS mechanisms and connectivity diagram. .165

Figure 5.6. Nominal, "Step Late”, and "Step Early" foot path diagrams. .168 
Figure 5.7. Stance speed acceleration from $0-50 \%$ of swing speed with identical joint actuator speeds, and starting ThC angles (relative to a Cartesian plane) listed in the table below .173

Figure 5.8. Six tests with stance speed acceleration from $0-50 \%$ of swing speed, and fixed starting ThC angles (relative to a Cartesian plane) listed in the tables below .175

Figure 5.9. Variations in iPEP positions induce turning 177

Figure 5.10. Composite images of the body and legs with fixed 50 degree foot paths for right legs, and decreasing foot path lengths for left legs. 178

Figure 5.11. Swing/stance plots of the turning trials with foot path lengths as follows .181

Figure 5.12. Robustness of BILL-LEGS to variations in a) mechanism setpoints, b) starting leg positions, and c) leg movement speeds

Figure 6.1. Early version of the Two-Leg Test Platform. .185

Figure 6.2. Series of images showing the fourth version of the Two-Leg Test Platform following a light source located toward its left. 188

Figure 6.3. Right-rear foot with replacement ground contact switch.

Figure 7.1. BILL-Ant-p2 right leg typical stepping motion foot path.

Figure 7.2. BILL-Ant-p2 leg joint angles and ground contact states 200

Figure 7.3. BILL-Ant-p2 right-leg foot path during a searching reflex .202

Figure 7.4. BILL-Ant-a left-front and right-front foot paths while walking on a flat surface. .206 
Figure 7.5. BILL-Ant-a gap crossing scenario to test the searching reflex.

Figure 7.6. BILL-Ant-a performing the elevator reflex.........................................209

Figure 7.7. BILL-Ant-a right-front foot paths during an elevator reflex.....................210

Figure 7.8. BILL-Ant-a performing goal searching behavior...................................211

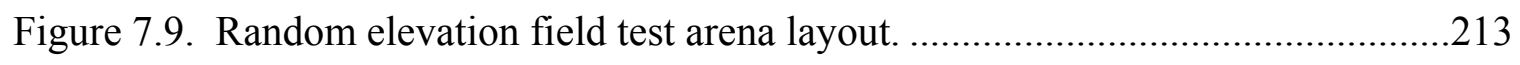

Figure 7.10. BILL-Ant-a in the random elevation field test arena. ............................214 


\section{ACKNOWLEDGEMENTS}

Julie Kleinhenz, my wife and friend, for being a source of constant support, even when I made no sense or was being crazy; and for kicking me when I needed it, but didn't realize.

Arnold and Jean Lewinger for nurturing my inner evil genius when as I was growing up (which I'm still in the process of doing).

Roger Quinn for being so supportive throughout my graduate career. He became my coadvisor in title, but has been the primary source of aid and understanding.

Brandon Rutter for cheerfully being a frequent sounding board for ideas and theories and a great person to work with. His support allowed this work to become much more cohesive and well though out.

Brian Taylor for also being a go-to person when theorizing new ideas and needing someone to talk to. Additionally, his review of this dissertation in its early draft stages was incredibly helpful in getting it all done.

Ansgar Büschges and Marcus Blümel for introducing me to their research, which is the basis for the SCASM controller. Conversations and interaction with them gave me a better understanding of stick insect neurophysiology.

Barbara Webb, for tolerating my work on this project while also working at my postdoctorate position for the University of Edinburgh.

The Biorobotics Lab students not mentioned above for all their help and support:

$\begin{array}{lll}\text { Rich Bachmann } & \text { Alex Boxerbaum } & \text { Kati Daltorio } \\ \text { Phil Dunker } & \text { Andy Horchler } & \text { Alex Hunt } \\ \text { Nicole Kern } & \text { Terence Wei } & \text { Tim Witushynsky }\end{array}$

The IGERT-NSF fellowship and NASA Glenn Research Center grant for providing the funding that made pursuit of this degree this possible. 


\section{LiST OF ABBREVIATIONS}

ADC

AEP

$\mathrm{AEPa}$

ANN

BILL-Ant-a

BILL-Ant-p

BILL-Ant-p2

BILL-LEGS

CTr, CT Joint

DoF

FK

FSR

FTi, FT Joint

GA

$\mathrm{I} / \mathrm{O}$

iAEP

$\mathrm{I}^{2} \mathrm{C}, \mathrm{I} 2 \mathrm{C}, \mathrm{IIC}$

\section{Analog-to-Digital Converter}

Anterior Extreme Position; the point during a stepping motion where the leg transitions from swing phase to stance phase

Adjusted Anterior Extreme Position

Artificial Neural Network

Biologically-Inspired Legged Locomotion - Ant - autonomous

The current hexapod robot presented in this dissertation with 19 active DoF and 12 passive DoF

BILL-Ant-prototype

The original 22 active DoF and 10 passive DoF hexapod robot with head and mandibles

BILL-Ant-prototype 2

Two-Leg Test Platform (7 active DoF and 4 passive DoF) with two neck-mounted light sensors; used to implement and test early SCASM control

Biologically-Inspired Legged Locomotion - Low computation power Emergent Gait System

Coxa-Trochanter Joint

Degree(s) of Freedom

Forward Kinematics

Force Sensitive Resistor

Femur-Tibia Joint

Genetic Algorithm

Input/Output

Intrinsic Anterior Extreme Position; the nominal AEP position, unmodified by any influences

Inter-IC Communication Interface (two-wire network protocol) 


$\begin{array}{ll}\text { IK } & \text { Inverse Kinematics } \\ \text { IR } & \text { Infrared } \\ \text { iPEP } & \begin{array}{l}\text { Intrinsic Posterior Extreme Position; the nominal PEP position, } \\ \text { unmodified by any influences }\end{array} \\ \text { Li-ion } & \text { Lithium Ion } \\ \text { MIPS } & \text { Millions of Instructions per Second } \\ \text { NiMH } & \text { Nickel Metal Hydride } \\ \text { PEP } & \begin{array}{l}\text { Posterior Extreme Position; the point during a stepping motion where } \\ \text { the leg transitions from stance phase to swing phase }\end{array} \\ \text { PEPa } & \text { Adjusted Posterior Extreme Position } \\ \text { PWM } & \text { Pulse Width Modulation } \\ \text { R/C } & \text { Radio Control, Radio Controlled } \\ \text { RT-Linux } & \text { Real-Time Linux Operating System } \\ \text { SCASM } & \text { Sensory-Coupled Action Switching Modules } \\ \text { ThC Toint } & \text { Thorax-Coxa Joint (also referred to as the Thoraco-Coxal Joint) } \\ & \end{array}$




\section{GLOSSARY}

BrainStem

See BrainStem Microcontroller.

BrainStem Microcontroller (BrainStem)

Programmable microcontroller with four R/C servo ports, five ADC input ports, five digital I/O ports, and RS-232 and $\mathrm{I}^{2} \mathrm{C}$ interfaces, used to control each of the legs and process "head" sensory information.

Coxa-Trochanter Joint (CTr Joint, CT Joint, Lift Joint)

The second (middle) joint and degree-of-freedom for each leg. Connecting the Lift Bracket and the femur, it consists of a horizontally-mounted R/C servo motor and controls leg lift.

CTr Joint, CT Joint

See Coxa-Trochanter Joint.

Femur

Leg segment between the body and knee. It consists of two parallel carbon fiber plates separated by an aluminum strut, and is shaped like a double yoke.

Femur-Tibia Joint (FTi Joint, FT Joint, Knee Joint)

The third (most distal) joint and degree-of-freedom for each leg. Connecting the femur and the tibia, it consists of a horizontally-mounted $\mathrm{R} / \mathrm{C}$ servo motor and controls knee bending.

FK

See Forward Kinematics.

Foot

Two parallel aluminum plates housing a force-resistive sensor for measuring leg loads, and is attached to the distal end of the tibia. 
Force Sensitive Resistor (FSR)

A device used to sense applied force. As force is applied, the sensor deforms, thereby changing the resistance as sensed at the output pins.

Forward Kinematics

A mathematical methodology used for determining the location of an assembly's end effector in space. The method uses the assembly geometry (shape and size of links, location of joints, link paths during actuation, etc.) and position of each joint to sequentially calculate segment locations from the proximal base to the end effector.

FSR

See Force Sensitive Resistor.

FTi Joint

See Femur-Tibia Joint.

IK

See Inverse Kinematics.

Inverse Kinematics

A mathematical methodology used for determining the joint angles within an assembly necessary to place the end effector at a desired location in space. The method uses the assembly geometry (shape and size of links, location of joints, link paths during actuation, etc.) and desired end effector position to determine the required joint angles.

Knee Joint

See Femur-Tibia Joint.

Leg Control Microcontroller

See BrainStem Microcontroller. 


\section{Lift Bracket}

An aluminum housing that holds the $\mathrm{CTr}$ Joint $\mathrm{R} / \mathrm{C}$ servo motor and provides a connection point for the ThC Joint.

\section{Lift Joint}

See Coxa-Trochanter Joint.

Microcontroller

See BrainStem Microcontroller.

Obstacle Contact Switch

A contact switch mounted at the end of each tibia that is used to detect collisions with raised obstacles.

Pulse Width Modulation (PWM)

A method of adjusting the average dc voltage by varying the amount of time that voltage is maximum, and zero otherwise, within a fixed period of time. For $\mathrm{R} / \mathrm{C}$ servos, a special type of PWM is used with a period of $2-50 \mathrm{msec}$. During the period, voltage is set to maximum (e.g. $6.0 \mathrm{vdc}$ ) for between $0.9 \mathrm{msec}$ and $2.1 \mathrm{msec}$ to achieve the full range of motion of the servo. The width of the pulse directly corresponds to the commanded output position of the servo, where a $1.5 \mathrm{msec}$ pulse represents the neutral or middle position.

R/C Servo

See R/C Servo Motor.

R/C Servo Motor (R/C Servo, Servo Motor, Servo)

Standard-size MPI MX-450HP radio control de servo motor, used in all joints on the robot.

Servo

See R/C Servo Motor.

Servo Motor

See R/C Servo Motor. 
Swing Joint

See Body-Coxa Joint.

ThC Joint, TC Joint

See Thorax-Coxa Joint.

Thorax

This is the main portion of the robot. It houses the batteries, leg control microcontrollers, and "head" sensory system and microcontroller. It also provides connection points for the ThC Joints.

Thorax-Coxa Joint (ThC Joint, TC Joint, Swing Joint)

The first (most proximal) joint and degree-of-freedom for each leg. Connecting the body and Lift Bracket, it consists of an inverted-mounted $\mathrm{R} / \mathrm{C}$ servo motor and controls leg swing.

Tibia

Distal leg segment between the knee and foot; made of aluminum. 


\section{Neurobiologically-based Control System for an Adaptively Walking Hexapod}

\section{Abstract}

by

\section{WILLIAM ANTHONY LEWINGER}

Biological systems such as insects have often been used as a source of inspiration when developing legged robots. Insects are capable of nimbly navigating uneven terrain. This ability, combined with their observed behavioral complexity has made them a beacon for engineers, who have used behavioral data and hypothesized control systems to develop remarkably agile robots. Beyond pure inspiration, it is now becoming possible to directly implement models of relatively recent discoveries in insect nervous systems in hexapod robot controllers. Specifically, walking control based on a model of a network discovered in the stick insect's thoracic ganglia, and not just observed insect behavior, has now been implemented in a complete hexapod that is able to walk, perform a goalseeking behavior, and obstacle surmounting behaviors such as single limb searching and elevator reflexes. Descending modulation of leg controllers is also incorporated via a "head module" that modifies leg controller parameters to accomplish turning in a role similar to the insect's higher centers. While many of these features have been previously demonstrated in simulation and with robotic subsystems, such as single- and two-legged 
test platforms, this is the first time that these neurobiological methods of control have been implemented in a complete, autonomous walking hexapod.

Many of these abilities have also been incorporated in previous hexapods by using more traditional engineering methods and methods based on external observations of insects. However, the methods described and used in this research, which are based on the actual neurobiological circuits found in the insect, are far simpler and therefore have much lower computational requirements. The reduced computation requirements lend themselves to small robots with limited on-board space available for the high-end processors needed for previous control methods.

This dissertation discusses the implementation of the biologically-grounded insect leg control method, descending modulation of that method, and the generation of stable, speed-dependent gaits. It then describes and quantifies the performance of the robot while navigating irregular terrain and performing phototaxis. Implementation is performed on the Biologically-Inspired Legged Locomotion - Ant - autonomous (BILLAnt-a) hexapod robot. 


\section{CHAPTER I}

\section{INTRODUCTION}

\subsection{General}

The goal of this work was to create an adaptively walking hexapod robot that was power and control autonomous and could locate and walk toward goals. As a basis for the work, it was decided to improve upon the previous research of the Biologically-Inspired Legged Locomotion - Ant - prototype (BILL-Ant-p) project (Lewinger 2005; Lewinger, Branicky, and Quinn 2005), which began in 2003 and concluded in 2005. BILL-Ant-p was a relatively small, legged hexapod that could manipulate objects with its mandibles and employ active compliance to minimize external forces. It was strong, in that it could walk with a payload of roughly 1.5 times its body weight, and could also walk on slightly uneven terrain.

Two major drawbacks of BILL-Ant-p was its extremely slow walking speed and its need for an off-board computer, which handled sensory processing and control of the 22 actuators. It was hypothesized that the computationally-intensive control methods and data communication bottleneck caused by the serial cable link between the robot and its off-board host computer were responsible for its slow movements.

The research described in this thesis covers the methods and results associated with a new control methodology that removed the two major drawbacks of BILLAnt-p. The new intra-leg joint control (used to create stepping motions) and inter- 
leg gait generation methods were developed to be computationally simple such that they could be performed by small, low-end microcontrollers that would fit on the small hexapod and provide control autonomy. Additionally, it was intended to provide the robot with a much more robust and adaptive style of walking that would allow navigation of highly irregular terrain, while still maintaining the computational simplicity of the control method.

\subsection{Background}

Completed in 2005, it was the goal of the Biologically-Inspired Legged Locomotion - Ant - prototype (BILL-Ant-p) project to develop a robot that was power and control autonomous; capable of: navigating uneven terrain, manipulating objects within the environment, and employment of compliance with the environment and other robots; very strong for its size; and relatively inexpensive compared to other similar robots. Some, but not all of the desired goals were met.

At the end of the project, BILL-Ant-p was power autonomous, but required an off-board computer for control. The connection between the host computer (System Controller) and the robot was responsible for the transmission of a large amount of data: sensory signal values being sent to the host, and servo motor command signals for 22 actuators being sent to the robot. This level of data flow caused a communications bottleneck that ultimately diminished the performance of BILL-Ant-p by causing slow, sluggish actions. 
At the time, there was no obvious solution to improving performance. While it was noted that an on-board computer would solve the communications issue, there was no processing device that was both powerful enough to handle the mathematically-intensive control tasks and that could also fit in the relatively small robot. This is one of the limitations of small-scale robots; they frequently have either simple behaviors due to small, low computation-capable on-board controllers, or they require off-board computing for more advanced control actions.

While BILL-Ant-p borrowed from nature in its appearance to a leaf-cutter ant (Hölldobler and Wilson 1990; Yahya 2000) and its method of leg coordination, which was similar to stick insects (Cruse et al. 1990), the robot used an engineering method to generate stepping motions. This method involved inverse kinematic equations to calculate the joint angles necessary to place the foot at a desired point in the work space of the leg. As expected, this method was computationally intensive and required trigonometric functions and floating-point math. It was this method that dictated the computation requirements for an onboard controller.

In 1997, Dietmar Hess and Ansgar Büschges (Hess and Büschges 1997) published a paper that detailed findings of the stick insect sensorimotor pathways involved in the joint motions required to generate stepping actions. This work showed that, in the stick insect, individual leg joints are controlled by localized oscillator circuits in the thoracic ganglia that use the influence of sensory information from 
local and neighboring joints in the same leg to generate stepping actions. This decentralized, reactive behavior implies that an emergent and sensory-influenced, rather than a purely pattern generator-defined, series of motions creates the movements necessary to form legged stepping in this insect.

Later, in 2004, Örjan Ekeberg, Marcus Blümel, and Ansgar Büschges (Ekeberg et al. 2004) developed a simulation that implemented the findings of the stick insect investigation as a set of rules that determined when each joint in the leg should change from flexion to extension, and from extension to flexion. As found in the insect, the simulation used a series of two-state systems for each of three leg joints (a 3-DoF model of a leg) that switched between flexion and extension based on the sensory inputs within the leg. Information such as joint load, change in load, and joint angle caused each of the three joints to change its direction of motion. By mechanically coupling the joints through the physical structure of the leg itself, these simple sensory connections caused a stepping motion to emerge.

This publication was a major find for the control of legged robots. Because the method presented a computationally simple means of generating stepping motions, it was assumed that it could be implemented on small, low computationcapable microcontrollers that would fit on a small robot such as BILL-Ant-p. The addition of new sensory paths was also theorized to provide an elevator reflex (stepping over a raised obstacle that was encountered) and a searching reflex (stepping over a discovered gap) (Lewinger, Rutter, et al., 2006). What remained to complete the locomotion system for a hexapod was to implement a means to 
coordinate the stepping actions of each limb in order to form gaits, as the neurobiological pathways responsible for this have not yet been discovered.

Cruse's method (Cruse 1990; Dean 1991a) of leg coordination has been used in several legged robots, including Robot I (Espenschied et al. 1993), Robot II (Espenschied et al. 1996), Tarry I and Tarry II (Frik et al. 1999; Cruse et al. 2003), the TUM Walking Machine (Pfeiffer et al. 1994; Weidermann et al. 1994), and BILL-Ant-p. Both Robot II and BILL-Ant-p used a reduced set of rules and chose only three of the seven mechanisms, which was the minimum number found to enable walking on non-sparse terrain while still generating a speeddependent continuum of gaits (Espenschied et al. 1996).

The way in which Cruse's coordination method was implemented for Robot II and BILL-Ant-p showed that the method had limited robustness to leg geometry, mechanism weight values, and starting pose (Lewinger and Quinn 2008). Additionally, a strategy such as a genetic algorithm (GA) was needed to choose the twelve mechanism values for BILL-Ant-p (eight unique values were required for Robot II). The values selected by the GA were quite specific and required decimal notation. As such, this method was not implementable on the low computation-capable microcontrollers that could support the new method of leg control.

By using Cruse's rules as a basis, a simplified means of multi-leg coordination was developed (Lewinger and Quinn 2008). This new method uses two mechanisms and two system-wide parameters (one for each mechanism) that 
enable a continuum of speed-dependent gaits to form, can be represented as integer values, and requires a small amount of addition and multiplication operations. Additionally, the simplified method appeared, in simulation, to be highly robust to starting pose and joint speeds. It was also shown in simulation that this method never allowed two adjacent legs to be in swing (unloaded) at the same time, thereby ensuring stability of the robot.

By combining the new means of generating steeping actions and coordinating leg stepping cycles, the BILL-Ant-p electronics were redesigned to use individual small, low computation-capable microcontroller to control each leg (six microcontrollers in all) coupled in a distributed network. The communication between adjacent legs provided the necessary coordination to form stable gaits while not requiring an over-arching controller.

A seventh, identical microcontroller was added to capture and process stereo sensory information in the form of infrared light (IR) that was used to implement phototropic behavior. This additional microcontroller acted as a "head" and modulated the actions of the six legs based on sensed light-based goals.

The amalgam of new microcontrollers, leg control and coordination methods, and modulation-implemented phototropic behavior became BILL-Ant-a Biologically-Inspired Legged Locomotion - Ant - autonomous (Fig. 1.1). While many of these features have been previously demonstrated with robotic subsystems, such as single- and two-leg test platforms, and as a hexapod in simulation only, this work represents the first time that the network responsible 
for leg control discovered in an insect's nervous system, rather than just derived from observed behaviors, has been implemented in a complete, autonomous walking hexapod. With the updates made to the prototype robot, BILL-Ant-a is both power and control autonomous, capable of navigating uneven and partially sparse terrain, and seeking out light-based goals (Lewinger and Quinn 2010a; Lewinger and Quinn 2010b).

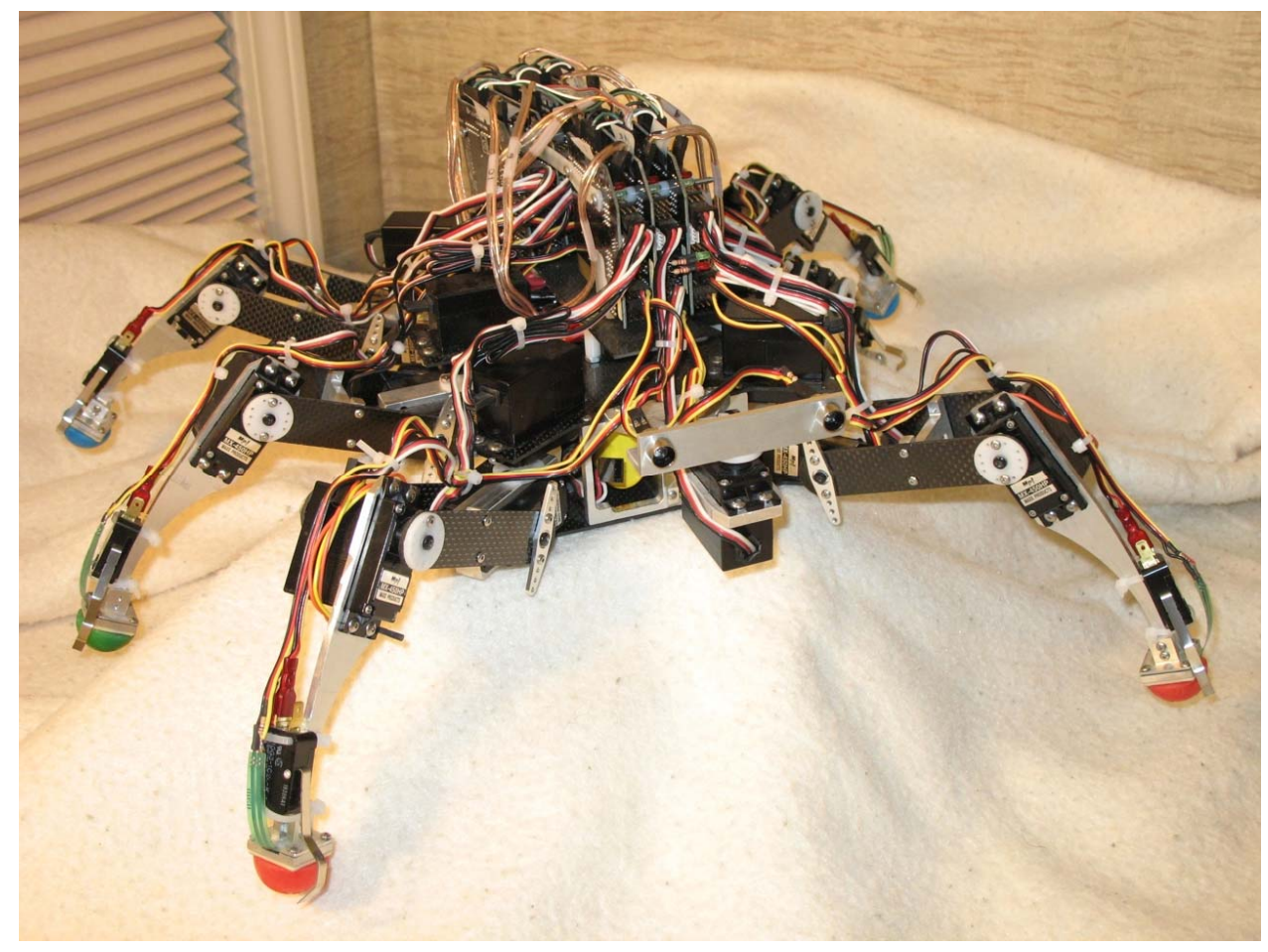

Figure 1.1. The Biologically-Inspired Legged Locomotion Ant - autonomous (BILL-Ant-a) robot.

\subsection{Goals}

The goals of this project were to update the BILL-Ant-p robot in order to create BILL-Ant-a. This new version was designed and created to:

- Be both power and control autonomous 
- Use on-board controllers to generate stepping motions for the legs

- Use on-board controllers to coordinate leg stance/swing cycles to generate speed-dependent gaits

- Provide robust stepping actions necessary to navigate irregular terrain

- Implement an elevator reflex to surmount climbable obstacles

- Implement a searching reflex to navigate gaps

- Autonomously identify goals and alter the robot's heading to intercept them

- Achieve the above goals with small, low computation-capable microcontrollers

\subsection{Thesis Contributions}

This thesis contributes the following:

- Formalizes a means of generating stepping motions for legged robots based on neurobiologically-identified circuits within insects (the SCASM method) that can be implemented on low computation-capable microcontrollers

- Adds the functionality of reflexes to the leg control method, which allows the legs to navigate past obstacles

- Creates a means of generating speed-dependent emergent gaits that is robust to starting pose and joint speeds and can be implemented on low computation-capable microcontrollers (BILL-LEGS) 
- Creates a modular, hierarchical control structure that allows for high-level modulation of the walking system. This infrastructure allows sensory, navigation, and learning systems to alter walking behaviors without the need for systems to handle the low-level management of walking.

- Implements SCASM and BILL-LEGS in both simulation and physical 2and 6-legged walking platforms through a network of distributed, low computation-capable microcontrollers

- Represents the first implementation of the SCASM leg control method in a physical hexapod.

- Verifies in simulation and physical implementation that modulation of SCASM parameters (stage change threshold setpoints and "muscle activation" values) can alter foot path heading and foot speed

- Verifies in simulation and physical implementation that stereoscopic sensing of a goal is sufficient to provide a useful input used for altering the walking direction of a 2- and 6-legged robot by modulation of SCASM values

- Verifies in simulation and physical implementation that the BILL-LEGS method of leg coordination is sufficient for the generation of emergent, speed-dependent hexapod gaits while also showing that the method provides static stability to a hexapod robot

- Quantifies performance data for the BILL-Ant-a robot as an autonomous, goal-seeking 18-DoF hexapod capable of navigating irregular terrain

- Produces the following BILL-Ant series related publications: 
○ Degree-related publications: 2

(Lewinger 2011; Lewinger 2005)

○ Journal publications: 1 (Invited Publication)

(Lewinger and Quinn 2011)

○ Refereed conference papers: 6 (1 Award)

(Lewinger and Quinn 2010b; Lewinger and Quinn 2010a; Lewinger and Quinn 2008; Rutter, Lewinger, Blümel, et al. 2007; Lewinger, Rutter, et al. 2006; Lewinger, Branicky, and Quinn 2005b)

○ Refereed conference videos: 1

(Lewinger and Quinn 2009)

○ Conference posters: 7 (2 Awards)

(Lewinger, Rutter, and Quinn 2008; Rutter et al. 2008b; Rutter et al. 2008a; Quinn et al. 2007; Rutter, Lewinger, Taylor, et al. 2007b; Rutter, Lewinger, Taylor, et al. 2007a; Quinn et al. 2006; )

○ Popular press: 8

(Material World 2009; Lockwood 2009; LERN 2007; Abbott 2007; Buggin' with Ruud 2006; Graduate Student News 2006; "Like an Animal" 2005; "Creature Features" 2005)

- Produces the following additional publications:

○ Journal publications: 1

(Lewinger et al. 2009)

○ Refereed conference papers: 3

(Dunker et al. 2009; Lewinger, Watson, and Quinn 2006a; Lewinger, Harley, et al. 2005a)

○ Refereed conference videos: 2 (1 Award)

(Lewinger, Watson, and Quinn 2006b; Lewinger, Harley, et al. 2005b) 
○ Refereed conference abstracts: 2

(Lewinger, Dunker, and Quinn 2008; Lewinger, Dunker and Quinn 2007)

○ Conference posters: 5 (1 Award)

(Dunker et al. 2008b; Dunker et al. 2008a; Dunker et al. 2007;

Lewinger, Harley, et al. 2006; Harley et al. 2005)

○ Academic reports: 3

(Lewinger 2006b; Lewinger 2006a; Harley et al. 2004)

○ Popular press: 6

(Kickin' It with Kenny 2007; Nature Tech 2006; Kickin' It with Kenny 2006; Buggin' with Ruud 2006; John Lydon's Megabug 2004)

\subsection{Chapter Topics}

Chapter 1, this chapter, is the introduction and discusses the research background, goals, contributions, and a list of topics for subsequent chapters.

Chapter 2 discusses desirable robot characteristics; reviews and compares similar robots; and describes previous methods for generating stepping motions and coordinating the actions of multi-legged robots to form stable gaits.

Chapter 3 covers the design and implementation of the mechanical, electrical, and electronic aspects of the BILL-Ant-a robot.

Chapter 4 describes the theory and implementation of the SCASM method for generating stepping motions in multi-joint legs. 
Chapter 5 describes the theory and implementation of the BILL-LEGS method for generating emergent, speed-dependent hexapod gaits.

Chapter 6 outlines the testing scenarios and quantifies the performance of BILLAnt-a.

Chapter 7 describes recommended future work. 


\section{CHAPTER II}

\section{REVIEW OF PREVIOUS LEGGED RoBot RESEARCH}

\section{$2.0 \quad$ General}

Legged hexapod robots have the ability to move over uneven and discontinuous terrain with more agility than wheeled or tracked vehicles of a similar size (Raibert et al. 2008; Espenschied et al. 1996). Also, within the hexapod robot community, only robots with at least three degrees-of-freedom per leg are capable of placing feet over a large range of positions, and executing holonomic turning and strafing movements - where the robot can move in one direction while facing another - such as crabbing (1- and 2-DoF legs can only move in one or two dimensions with a severely limited range of foot placement options). While robots with fewer than 3-DoF are physically able to turn, they usually do so with either a form of skid steering (legs on one side of the body moving with different speeds and step lengths than those of the opposing side) or employ one or more steering joints that rotate either the leg base or the body segment attached to the legs, which adds degrees of freedom to the system; none of these methods are employed by animals and insects for turning (Dürr and Ebeling 2005).

\subsection{8-DoF Hexapod Robot Designs}

There are numerous hexapod robots with 3-DoF legs that have been created for research, hobby, and entertainment. The robots presented below were chosen for their physical design, method of actuation, and research intent. There are many 
more robots than are described below; however, the mentioned hexapods were selected because of their similarity to BILL-Ant-a.

While capable of successful navigation of uneven terrain, hexapod robots such as Whegs $^{\mathrm{TM}}$ II (Allen et al. 2003), Lunar Whegs ${ }^{\mathrm{TM}}$ (Dunker et al. 2007, Dunker et al. 2008, Dunker et al. 2009, Lewinger, Dunker, and Quinn 2008, and Lewinger, Dunker, and Quinn 2007), and RHex (Saranli et al. 2001) with 1-DoF legs are fundamentally different in design from BILL-Ant-a. Although they are more similar in design to BILL-Ant-a than 1-DoF per leg vehicles, robots such as Robot I (Espenschied and Quinn 1993) and Genghis (Brooks 1989) with 2-DoF legs are also not reviewed. Additionally, robots with more than 3-DoF per leg were not used for comparison since they are more complex, require extra amounts of motor power and control, and the additional joints are not necessary for basic navigation of uneven and discontinuous terrain. As such, only 3-DoF per leg hexapod robots are reviewed.

The hexapods discussed below have one or more of the desired features of BILLAnt-a, but not all. These features include: ability to navigate highly-irregular terrain; adaptive gait generation; low computational complexity control system; the ability to locate and walk toward goals; simply actuated joints; on-board power and control; small size. 


\subsubsection{TUM Walking Machine}

Dr. Friedrich Pfeiffer at Technische Universität München began work on the TUM Walking Machine (Fig. 2.1) in 1991 (Pfeiffer et al. 1994; Weidermann et al. 1994). This robot is based on the stick insect and uses a form of Cruse control for leg coordination (Pfeiffer et al. 1994). The TUM Walking Machine uses distributed leg control so that each leg may be self-regulating with influences from adjacent legs.

Unlike other robots discussed below that use the Cruse rules for leg coordination, the TUM Walking machine only uses Mechanism 1 from the Cruse model: "A leg is hindered from starting its return stroke while its posterior leg is performing a return stroke" (Barnes 1998) and is applied to ipsilateral and contralateral

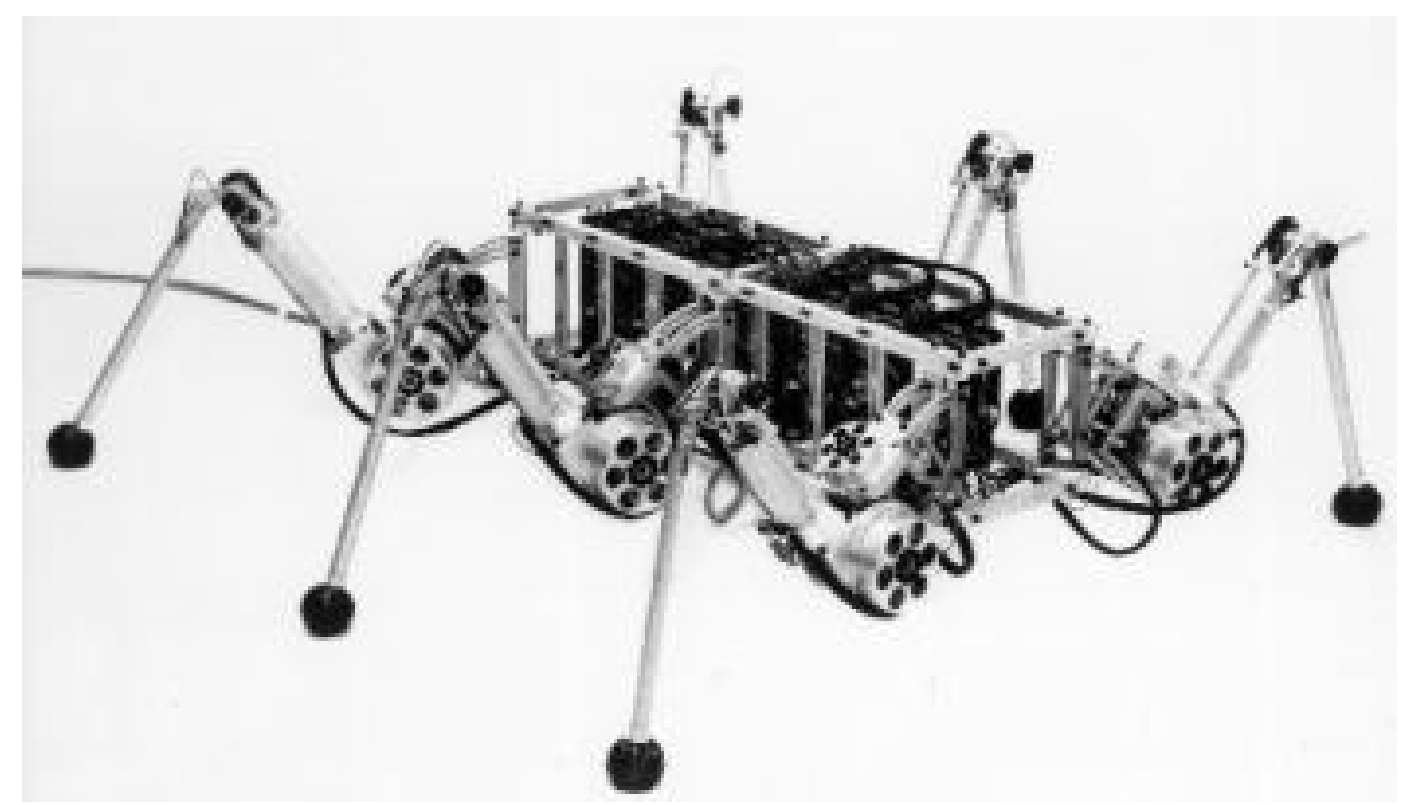

Figure 2.1. TUM Walking Machine

\section{Built by Dr. Friedrich Pfeiffer at Technische Universität München (Berns 2005).}


adjacent legs. The original Cruse model only applies the influences of Mechanism 1 to ipsilateral rostral legs (Fig. 2.19). Additionally, a leg is prevented from entering its swing phase while any adjacent neighboring legs were also in swing. This is an enforcement of static stability, which differs from Cruse's 'suggestion'-style influence system and results in the method only producing an alternating tripod gait, rather than a continuum of gaits.

The TUM Walking machine is $31.5 \mathrm{in}(80 \mathrm{~cm})$ long, $39.4 \mathrm{in}(100 \mathrm{~cm})$ wide, and 15.7in $(40 \mathrm{~cm})$ tall (Berns 2005$)$.

\subsubsection{Tarry I and Tarry II}

Based on the stick insect (Carausius morosus), development of Tarry I (Fig. 2.2, left) began in 1992 by the Department of Engineering Mechanics at the University of Duisberg, and was headed by Dr. Martin Frik (Frik et al. 1999). The goal was to develop an autonomous six-legged walking vehicle to navigate smooth and uneven terrains while under operator control, and to autonomously explore and determine what path to take when moving to a pre-defined goal (Buschmann 2000a). Similar to the BILL-Ant-a robot, Tarry I uses hobby R/C servo motors for the leg joints and has 18 degrees-of-freedom.

In 1998, development of Tarry II (Fig. 2.2, right) began. Tarry II is similar to Tarry I, but more loosely based on the stick insect. It uses more powerful servo motors and some other slight design changes that offer better mechanical advantages for the legs. While Tarry I uses stick insect proportions for relative 
leg placement and segment lengths, Tarry II uses dimensions that reduce mechanical strain (Buschmann 2000b).

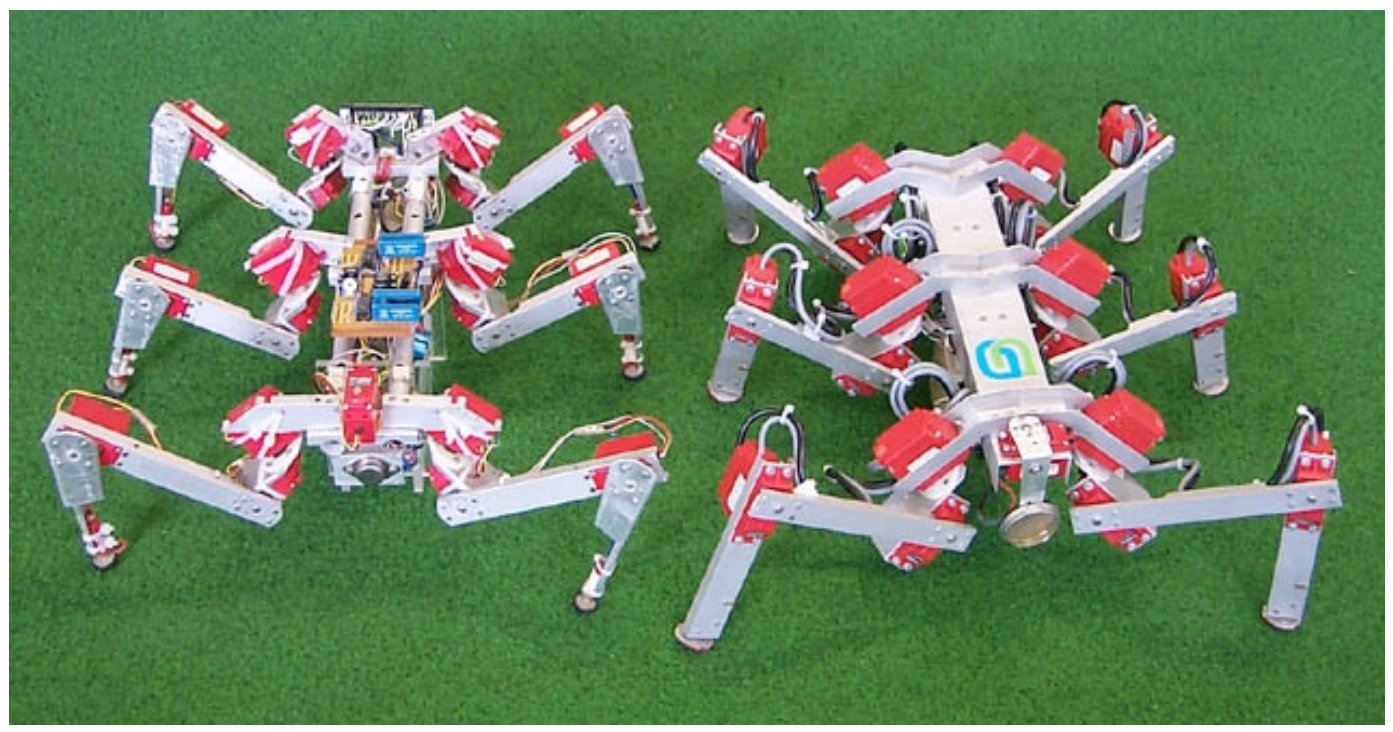

Figure 2.2. Tarry I (left) and Tarry II (right)

\section{Built by the Department of Engineering Mechanics at the University of Duisberg (Buschmann 2000a).}

Tarry I and Tarry II also have foot contact switches, instead of the force-sensing resistors (FSRs) used in the BILL-Ant-a and BILL-Ant-p robots. Power consumed by the leg actuators is measured to detect collisions with obstacles. Also, a front-mounted ultrasonic sensor is used to detect large obstacles. The robots also employ strain gauges attached to the legs for measuring strains during movement, and an inclinometer used to maintain a horizontal body posture (Buschmann 2000b).

Located on an off-board computer, the Tarry series uses an implementation of the Cruse controller called Walknet, an artificial neural network (ANN) (Cruse et al. 1993; Cruse et al. 2003). Walknet is trained to control the joint motors in order to 
perform walking tasks such as straight walking at different speeds, walking in curves, and walking in directions different from the robot heading (strafing).

Tarry $\mathrm{I}$ is approximately $19.7 \mathrm{in}(50 \mathrm{~cm})$ long, $15.7 \mathrm{in}(40 \mathrm{~cm})$ wide, 7.1 in tall $(18 \mathrm{~cm})$, weighs $4.601 \mathrm{bs}(2092 \mathrm{~g})$, and can support a payload of $0.881 \mathrm{bs}(400 \mathrm{~g})$. Tarry II is approximately $19.7 \mathrm{in}(50 \mathrm{~cm})$ long, $19.7 \mathrm{in}(50 \mathrm{~cm})$ wide, 7.9 in tall $(20 \mathrm{~cm})$, weighs $6.39 \mathrm{lbs}(2905 \mathrm{~g})$, and can support a payload of $6.38 \mathrm{lbs}(2900 \mathrm{~g})$ (Buschmann 2000a).

\subsubsection{Robot II}

At the Case Western Reserve University Biorobotics Lab in 1993, development began on Robot II (Fig. 2.3), a stick insect-inspired, 18 active degrees-of-freedom (six passive DoF) hexapod robot (Espenschied and Quinn 1994; Espenschied et al. 1994; Espenschied et al. 1996). Robot II uses insect-inspired reflexes for each leg that allow the legs to navigate uneven and discontinuous terrain independently of one another.

The off-board gait controller for Robot II was adapted from the network published by Cruse and Dean, which was based on observed stick insect leg coordination (Cruse et al. 1993). A continuous range of statically-stable gaits is generated as speed increases over flat terrain. With a similar behavior to BILL-Ant-a, when encountering discontinuous terrain and obstacles, Robot II employs two insectlike behaviors to navigate successfully: a searching reflex and an elevator reflex, respectively (Espenschied et al. 1996). 


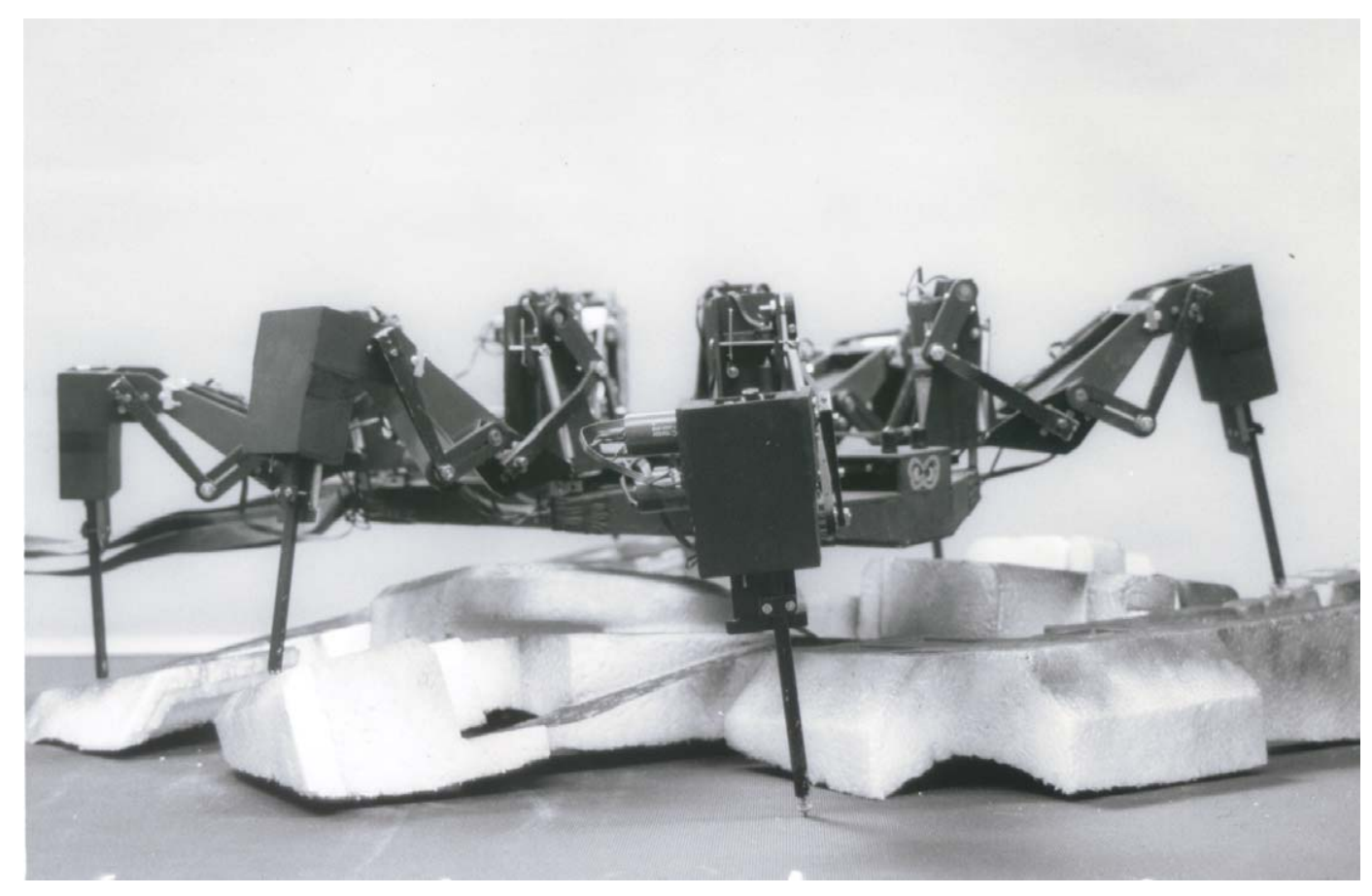

Figure 2.3. Robot II

\section{Built by the Biorobotics Lab in the Department of Mechanical and Aerospace Engineering at Case Western Reserve University (Espenschied et al. 1996).}

On discontinuous surfaces, such as a slatted walkway, the searching reflex is initiated when the passive force sensor in the tibia fails to detect the ground at the expected level. An iterative cycle of searching downward and forward for a foothold begins, where a pattern of ever-increasing radii circles is used for a fixed number of cycles.

If an obstacle is detected while walking, the elevator reflex is initiated and reverses the leg direction and proceeds forward again at a higher elevation. This process is repeated up to a height of $3.15 \mathrm{in}(8 \mathrm{~cm})$. 
Like the Tarry series, Robot II uses external power and an external leg control system. Unlike BILL-Ant-a and the robots previously mentioned, however, Robot II uses 18 6-Watt Maxon motors (Maxon Motor AG, Sachseln, Switzerland) and position feedback potentiometers for the actuated joints, instead of hobby servo motors, and employs an external motor control system to set position, velocity, and stiffness of each actuator, features of the control board found within hobby servo motors.

Robot II is 19.7 in $(50 \mathrm{~cm})$ long, 19.7 in $(50 \mathrm{~cm})$ wide, and 9.8 in $(25 \mathrm{~cm})$ tall (Espenschied et al. 1996).

\subsubsection{BILL-Ant-p}

Designed and constructed as part of a Master's degree in engineering at Case Western Reserve University, the Biologically-Inspired Legged Locomotion - Ant - prototype (BILL-Ant-p) robot was built to navigate irregular terrain, employ active compliance for posture, and be capable of manipulating objects with actively-compliant mandibles (Fig. 2.4) (Lewinger 2005; Lewinger, Branicky, and Quinn 2005). This robot most-closely resembles BILL-Ant-a as its chassis was used as the basis for the newer robot.

BILL-Ant-p has six, 3-DoF legs. Each leg has a thorax-coxa joint (ThC joint) responsible for moving the leg forward and rearward in the horizontal plane, a coxa-trochanter joint (CTr joint) that is used to levate and depress the leg, and a femur-tibia joint (FTi joint) capable of extending and flexing the tibia and foot 
assembly. The CTr and FTi joints are perpendicular to one another and act on each leg in the same plane (leg plane).

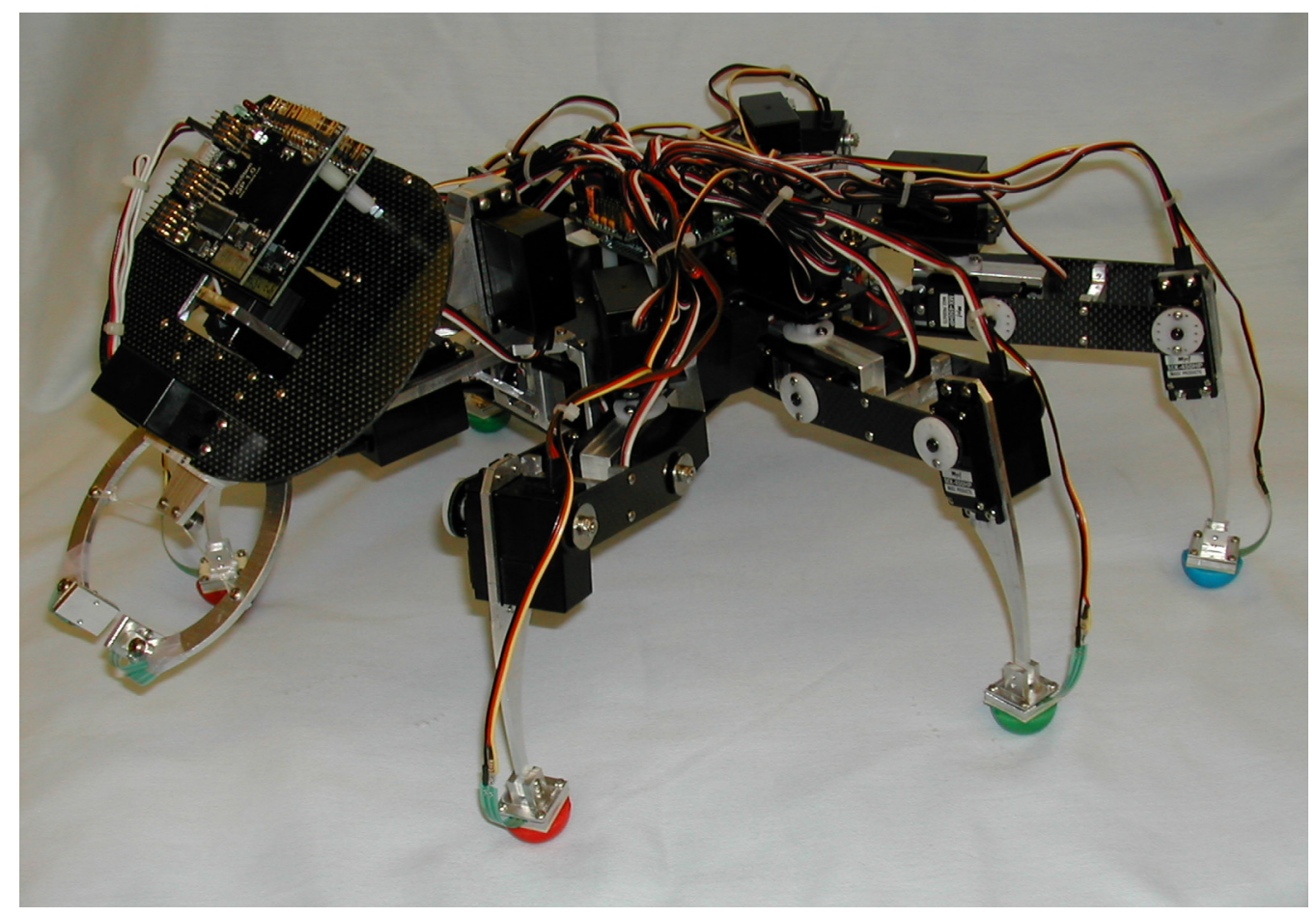

Figure 2.4. The Biologically-Inspired Legged Locomotion - Ant - prototype (BILL-Ant-p) robot.

The robot has six 3-Dof legs, a 3-DoF neck, 1-DoF set of mandibles, and 10 degrees of passive freedom (one per leg, two per mandible) (Lewinger 2005; Lewinger, Branicky, and Quinn 2005).

This robot uses four on-board 7.4vdc Li-Ion batteries each routed through a 6vdc voltage regulator. Three of the battery/regulator pairs are connected in parallel to provide power to the joint servo motors, while the fourth pair powers microcontrollers: one New Micros, Inc. (Dallas, TX, USA) IsoPod ${ }^{\mathrm{TM}}$ V2 SR microcontroller servo control board and two Acroname BrainStem GP 1.0 microcontrollers (Acroname, Inc., Boulder, CO, USA). The IsoPod ${ }^{\mathrm{TM}}$ servo controller transmits joint commands to the 18 servo motors in the legs and reads 
the six force sensitive resistors (FSRs) located in the feet. The two BrainStem microcontrollers are located on the head and are used to control the 3-DoF neck, actuate the mandibles, and read the four FSRs located in the mandible tips. The BrainStem units are connected to one another by an $\mathrm{I}^{2} \mathrm{C}$ bus. The IsoPod ${ }^{\mathrm{TM}}$ and BrainStem controllers are coupled to an off-board computer (System Controller) via two RS-232 serial cables: one for the IsoPod $^{\mathrm{TM}}$ and one for the pair of BrainStems.

The System Controller (laptop computer, 2.8MHz P4, 1GB RAM, 60GB HD) runs the command and control software that communicates with the on-board microcontrollers to produce movement and activity in the robot, and provides the operator with visual feedback on the status of the robot.

A Software Interface was created using Microsoft Visual Basic 6.0 (Microsoft Inc., Redmond, WA, USA). The graphical interface allows the operator to command robot actions and view robot status. Basic commands on the interface allow the operator to: manipulate each leg joint; set foot position in body-centric $\mathrm{x}-, \mathrm{y}-$, and $\mathrm{z}-$ coordinates; initiate a standing routine; adopt a standing posture; adjust body height from the ground; adjust body roll and pitch; drive the robot using speed, heading, and rotation values; and manipulate the neck and mandibles. Body, leg, and drive interfaces are shown in Fig. 2.5. 


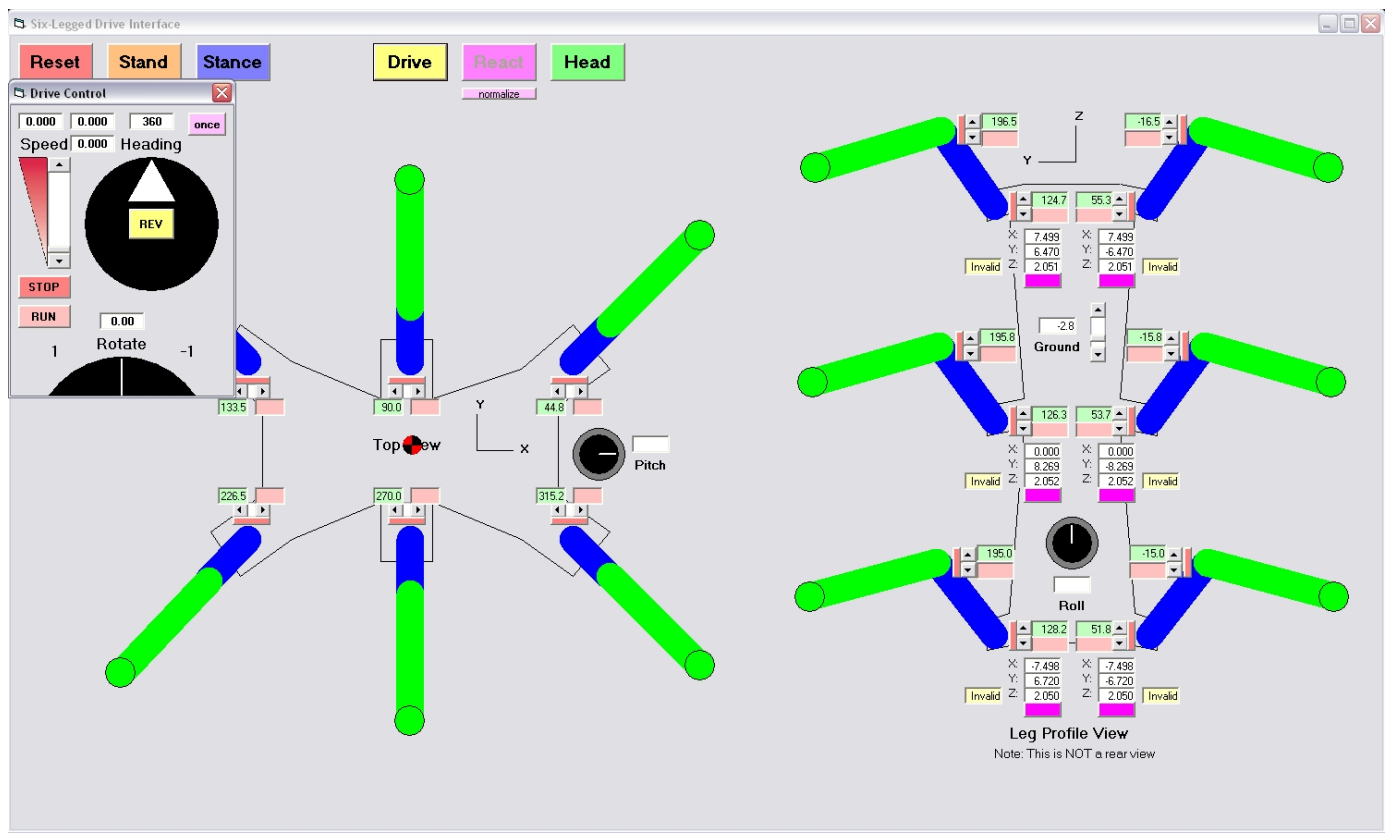

Figure 2.5. BILL-Ant-p user interface with Drive Control window shown.

Leg joint control is accomplished by using trigonometry-based inverse kinematics (IK) calculations in real-time that command the foot to follow a parabolic trajectory when in the swing phase, and a straight-line path during the stance phase. During each calculation cycle, joint motor positions are assigned such that the foot is positioned at points along the foot path. The foot path trajectories are modified autonomously based on foot sensor feedback (used to determine ground contact) and manually in response to user-defined heading and body rotation rate (yaw) settings.

Stance and swing phases of each of the six legs are coordinated to form speeddependent gait patterns based on Cruse's rules for leg coordination (Cruse 1990). Three mechanisms are used to couple ipsilateral and contralateral adjacent leg pairs. Mechanism 1 inhibits the start of the neighbor's swing phase when the current leg is already in its swing phase; Mechanism 2 excites the start of the 
neighbor's swing phase when the current leg begins its stance phase; and Mechanism 3 is a ramp signal that also excites the start of the neighbor's swing phase as the current leg propels along its step path. While Cruse's method employs seven mechanisms $(1-4,5 \mathrm{a}, 5 \mathrm{~b}$, and 6$)$, the additional mechanisms were not deemed necessary for walking on uneven, populated terrain and are not used (Espenschied and Quinn 1994; Espenschied et al. 1993; Espenschied et al. 1994; Espenschied et al. 1996) .

A main limitation of BILL-Ant-p is necessity of the off-board System Controller. Due to the communications bottleneck created by the RS-232 serial connections, and that the System Controller is performing trigonometric IK calculations live, the robot has a very slow maximum walking speed of $0.004 \mathrm{~m} / \mathrm{s}$. The movement performance and the lack of control autonomy are the main reasons for the development of BILL-Ant-a with on-board control.

\subsubsection{Lynxmotion Hexapod Kits}

Lynxmotion, Inc. (Pekin, IL, USA) has a variety of available robot kits (Fig. 2.6) and parts with different levels of actuation, articulation, control, and complexity. Kits such as the BH3 Walking Robot (Lynxmotion BH3 2008) and the Phoenix Hexapod Walking Robot (Lynxmotion Phoenix 2008) can be ordered as a complete set of parts with body pieces, servo motors, and electronics; or with different options, such as just the body pieces, or with alternate, more powerful servo motors. 

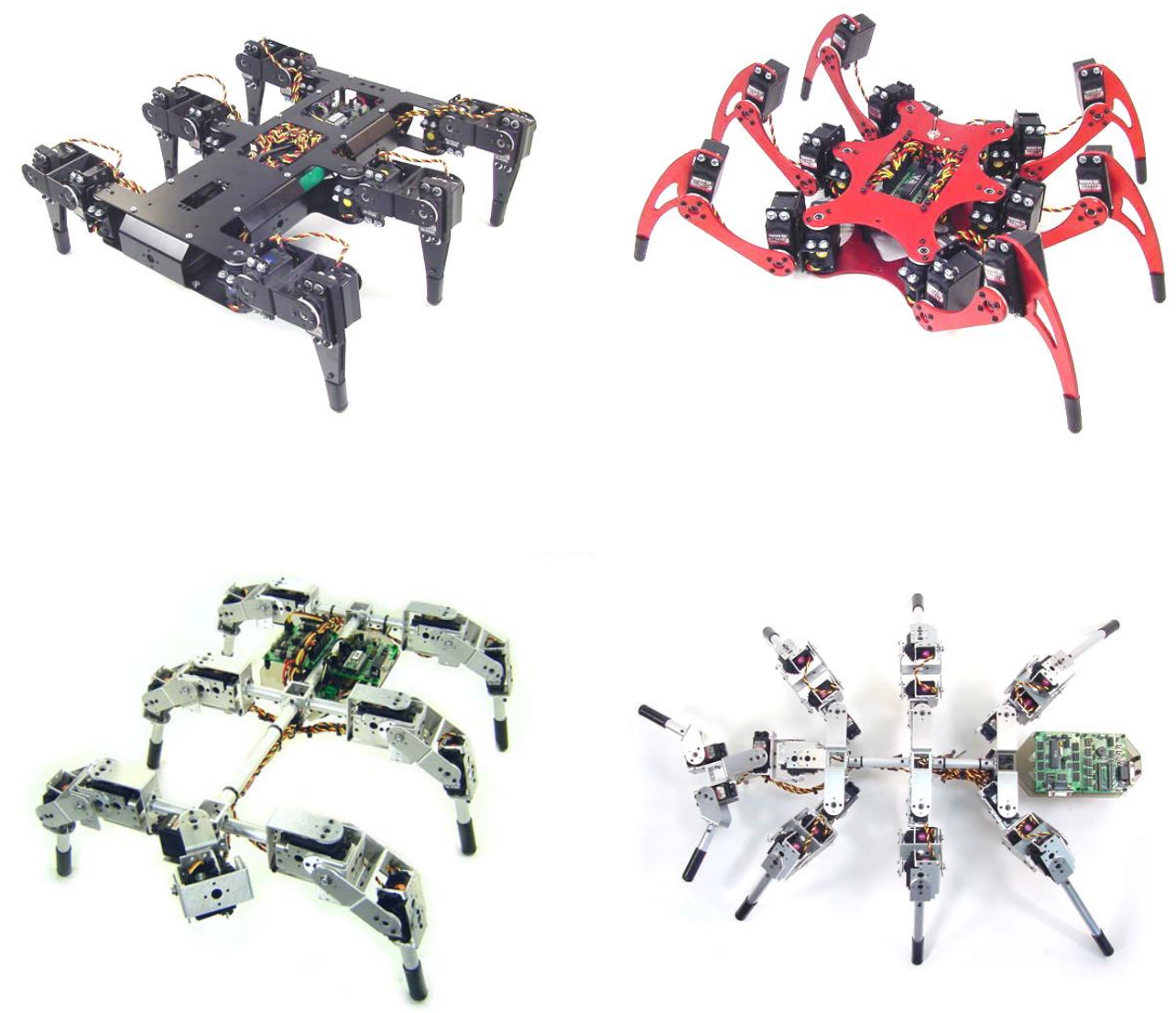

Figure 2.6. Collection of Lynxmotion, Inc. hexapod robots:

\section{BH3 (top left), Phoenix (top right), Walking Stick (bottom left), and "Robo-} Ant" (bottom right). The bottom two robots were constructed using the Servo Erector Set (Lynxmotion BH3 2008; Lynxmotion Phoenix 2008; Lynxmotion Servo Erector Set 2008).

Like BILL-Ant-p and robots mentioned previously, the Lynxmotion kits actuate leg segments by direct connection to hobby servo motors that are embedded in the legs. This allows for simplified leg mechanics and can also ease the complexity of joint control, as each leg is directly driven by its associated servo and not affected by angular offsets caused by mechanical coupling with other segments.

If none of the vast selection of legged robot kit options is suitable to a designer's needs or desires, Lynxmotion, Inc. also offers a product line called the Servo 
Erector Set (SES) (Lynxmotion Servo Erector Set 2008). This series of parts allows designers to choose pre-made, modular brackets, link segments, and other coupling pieces to use as construction elements in order to build custom robots and electro-mechanical assemblies. Electing to use the SES instead of a kit also allows builders to choose their own servo motors and control electronics. For robot creators without access to machining and fabrication facilities, the Servo Erector Set offers a simple option to built robots.

In July of 2005, I was contacted by Jim Frye (owner of Lynxmotion, Inc.). He indicated that he came across my master's degree thesis on BILL-Ant-p, was inspired to create his own design, and made the 3DOF Hexapod "Robo-Ant" (Fig. 2.6, bottom right). It was a wonderful compliment to my research.

The BH3 kit comes with the SSC-32 servo control board, which can control up to 32 hobby servo motors, and the Bot Board II carrier (BB2) housing a BasicATOM 28 microcontroller. This combination of electronics (identical to that found in the A-pod robot discussed in Section 2.1.5) allows the robot to use pre-programmed control and gaits for walking.

The Phoenix kit, as with several of the Lynxmotion kits, has several options, such as a black anodized or brushed aluminum chassis design, the choice of a full kit or mechanical parts only, and an optional wireless PlayStation2-style joystick controller. Command of the robot can be accomplished via the joystick device or connection with a host computer and a freely-available software interface called Visual Sequencer. As with most small-scale robot kits, the builder is able to use 
many different, off-the-shelf, electronic control hardware options, or design control electronics from scratch.

The BH3 Walking Robot is $14.5 \mathrm{in}(37 \mathrm{~cm})$ long, $14.0 \mathrm{in}(36 \mathrm{~cm})$ wide, and $3.5 \mathrm{in}$ (9cm) tall (Lynxmotion BH3 2008). The Phoenix Hexapod Walking Robot is $14.5 \mathrm{in}(37 \mathrm{~cm})$ long, $17.0 \mathrm{in}(43 \mathrm{~cm})$ wide, and $5.25 \mathrm{in}(13 \mathrm{~cm})$ tall (Lynxmotion Phoenix 2008). The Servo Erector Set (SES) can create robots of varying dimensions.

\subsubsection{AMOS-WD06}

Inspired by cockroaches, spiders, and scorpions, the Advance Mobility Sensor Driven-Walking Device 06 (AMOS-WD06) hexapod robot (Fig. 2.7) (Manoonpong et al. 2008) is mechanically similar to BILL-Ant-a. It has six, 3DoF legs that are constructed from aluminum frame-coupled hobby servo motors, where the servo motors directly drive the leg joints. AMOS-WD06 has additional points of movement due to an actuated thorax joint located between the middle and front legs that allows the thorax to bend up and down (like a body flexion joint seen in animals), and two actuated joints in the tail that can position the mounted wireless camera.

While power is supplied by an off-board source, sensor and control processing is accomplished with an on-board personal digital assistant (PDA). Artificial neural networks (ANNs) are programmed into the PDA to filter and process the sensory information and then control the joint motors via ANN oscillator circuits. 
Sensory signals are inputted and joint commands are outputted via an on-board Multi-Servo IO Board (Mboard) that has 36 analog-to-digital converter (ADC) ports and can control up to 32 servo motors. The PDA and Mboard are connected via an RS-232 serial cable communicating at $57.6 \mathrm{kbits} / \mathrm{s}$.

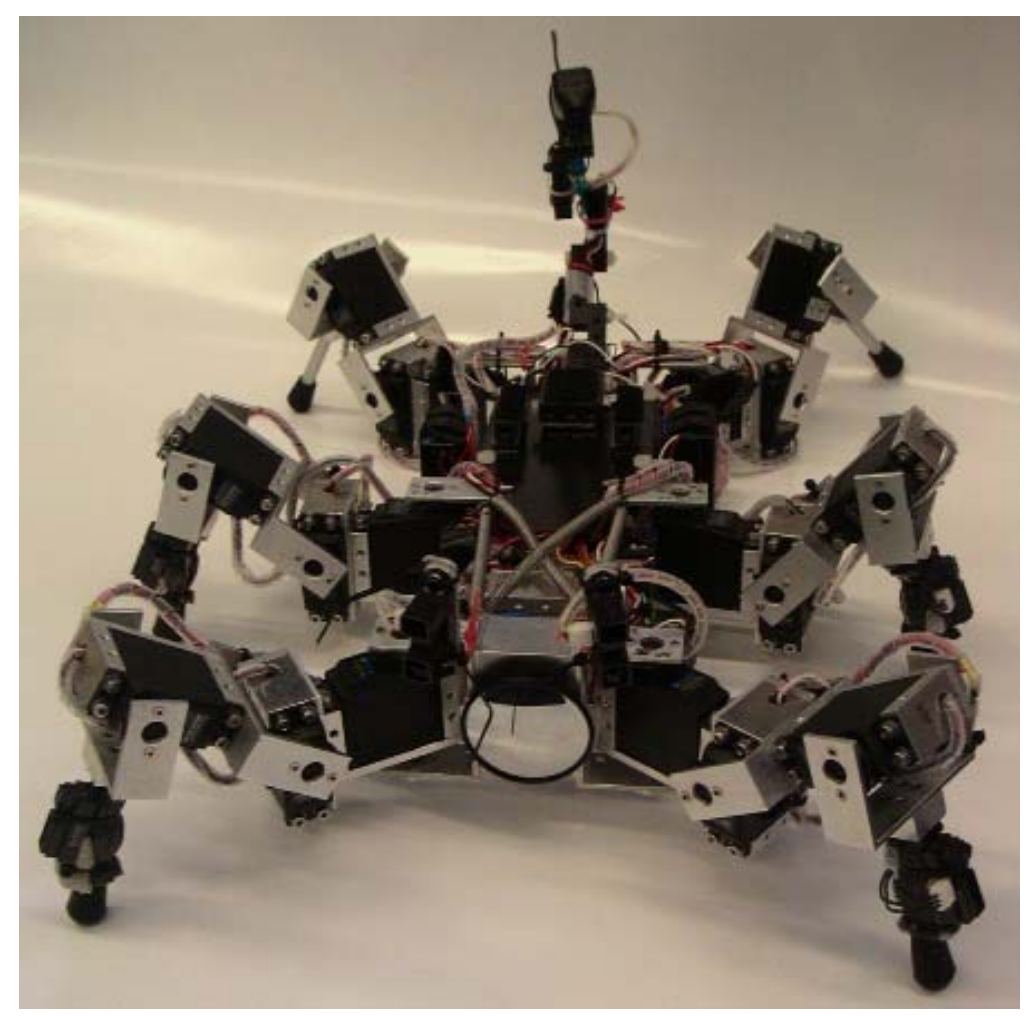

Figure 2.7. AMOS-WD06 is a hexapod robot designed by Poramate Manoonpong in 2006.

It has 3-DoF legs with an actuated point of bending in the thorax to aid in climbing. Two additional degrees of freedom are found in the tail, which supports a wireless camera (Manoonpong et al. 2008)

AMOS-WD06 has a host of sensors (infrared range sensors and an auditory sensor) that inform the robot of its environment and a number of behaviors that react to the sensory information. Extensions of the ANN filter and process sensory information and then initiate or modulate one the 11 walking patterns. 
Based on tropic and phobic behaviors found in animals, AMOS-WD06 is able to detect predator sounds and initiate a retreat, hear low frequency prey sounds and move toward them, and sense obstacles in order to avoid them. The sensory behaviors are integrated to form a composite decision on what action to take.

While standing at its maximum height, AMOS-WD06 is $15.7 \mathrm{in}(40 \mathrm{~cm})$ long, 4.70in $(12 \mathrm{~cm})$ wide, and $11.8 \mathrm{in}$ tall $(30 \mathrm{~cm})$. It weighs $9.24 \mathrm{lbs}(4200 \mathrm{~g})$ and can support a payload of $2.201 \mathrm{bs}(1000 \mathrm{~g})$.

\subsubsection{Micromagic Systems Hexapod Robot Series}

Matt Denton of Micromagic Systems (Winchester, Hanst, UK) has been involved in prop and set design, animatronics, and robotics for nearly 15 years. His hexapod series of robots are small-scale, 3-DoF per leg machines that move with a grace that appears lifelike (Fig. 2.8) (Denton 2008).

The seven hexapod robots listed in Table 2.1 each have 3-DoF legs that are actuated by hobby servo motors located at the joints. Each leg segment is attached to the output of the servo either by means of the servo horn, or by press fit. Earlier hexapod versions, V1 through V3, use micro-sized hobby servo motors (approximate servo dimensions - L: 1.14in, W: 0.51in, H: 1.18in; L: 29mm, W: 13mm, H: 30mm), while versions V4 and V4b use a mix of micro- and standard-sized motors. The larger BF Hexapod V5 uses high torque, standardsized Hitec HSR-5995TG servo motors (Hitec RCD, Inc., Poway, CA, USA) 
(servo dimensions - L: 1.57in, W: 0.78in, H: 1.45in; L: 40mm, W: 20mm, H: $37 \mathrm{~mm}$ ), and the i.C. Hexapod uses a different model of standard-sized servo.
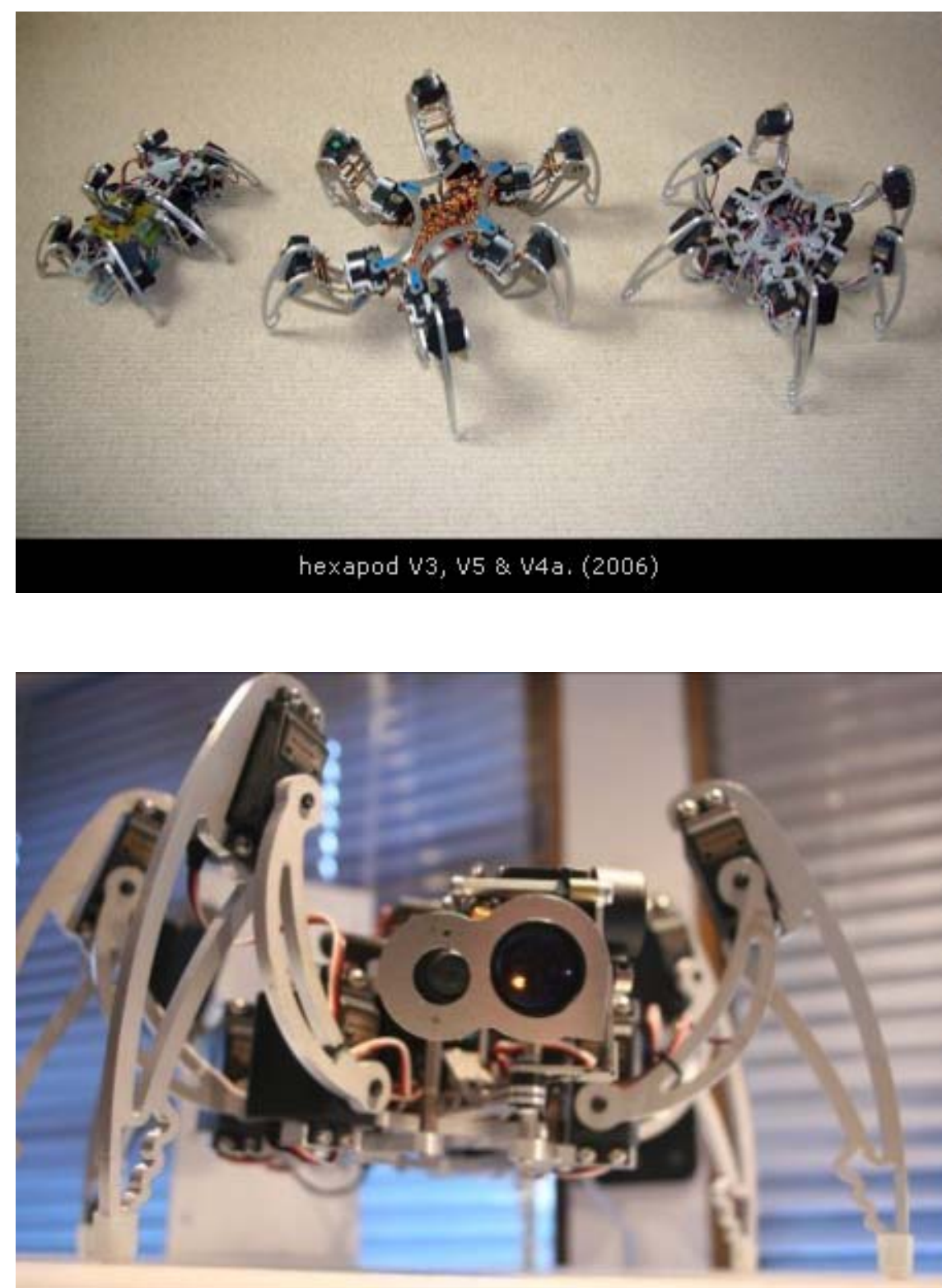

"i.c. hexapod" face tracking interactive hexapod (2007)

Figure 2.8. Collection of Micromagic Systems hexapod robots:

V3 (top left), V5 (top middle), V4 (top right), and i.C. (bottom) built by Matt Denton (Denton 2008). 
The Micromagic Systems hexapod robot line has the following characteristics:

\begin{tabular}{|c|c|c|c|c|c|}
\hline Robot Name & length & width & height & mass & payload \\
\hline $\begin{array}{c}\text { Hexapod V1 } \\
\text { PyE Hexapod) }\end{array}$ & $\begin{array}{c}12.6 \mathrm{in} \\
32 \mathrm{~cm}\end{array}$ & $\begin{array}{c}11.4 \mathrm{in} \\
29 \mathrm{~cm}\end{array}$ & $\begin{array}{c}7.9 \mathrm{in} \\
20 \mathrm{~cm}\end{array}$ & $1.3 \mathrm{~kg}$ & $0.4 \mathrm{~kg}$ \\
\hline Hexapod V2 & $\begin{array}{c}11.8 \mathrm{in} \\
30 \mathrm{~cm}\end{array}$ & $\begin{array}{c}11.0 \mathrm{in} \\
28 \mathrm{~cm}\end{array}$ & $\begin{array}{c}7.9 \mathrm{in} \\
20 \mathrm{~cm}\end{array}$ & $1.1 \mathrm{~kg}$ & $0.4 \mathrm{~kg}$ \\
\hline Hexapod V3 & $\begin{array}{c}11.8 \mathrm{in} \\
30 \mathrm{~cm}\end{array}$ & $\begin{array}{c}11.0 \mathrm{in} \\
28 \mathrm{~cm}\end{array}$ & $\begin{array}{c}7.9 \mathrm{in} \\
20 \mathrm{~cm}\end{array}$ & $1.1 \mathrm{~kg}$ & $0.5 \mathrm{~kg}$ \\
\hline Hexapod V4 & $\begin{array}{c}15.7 \mathrm{in} \\
40 \mathrm{~cm}\end{array}$ & $\begin{array}{c}11.8 \mathrm{in} \\
30 \mathrm{~cm}\end{array}$ & $\begin{array}{c}7.9 \mathrm{in} \\
20 \mathrm{~cm}\end{array}$ & $3.0 \mathrm{~kg}$ & $0.75 \mathrm{~kg}$ \\
\hline Hexapod V4b & $\begin{array}{c}15.7 \mathrm{in} \\
40 \mathrm{~cm}\end{array}$ & $\begin{array}{c}11.8 \mathrm{in} \\
30 \mathrm{~cm}\end{array}$ & $\begin{array}{c}7.9 \mathrm{in} \\
20 \mathrm{~cm}\end{array}$ & $1.8 \mathrm{~kg}$ & -- \\
\hline $\begin{array}{c}\text { Hexapod V5 } \\
\text { (BF Hexapod) }\end{array}$ & $\begin{array}{c}19.7 \mathrm{in} * \\
50 \mathrm{~cm}\end{array}$ & $\begin{array}{c}13.8 \mathrm{in} * \\
35 \mathrm{~cm}\end{array}$ & $\begin{array}{c}10 \mathrm{in} * \\
25 \mathrm{~cm}\end{array}$ & $3.3 \mathrm{~kg}$ & -- \\
\hline i.C. Hexapod & $\begin{array}{c}15.7 \mathrm{in} \dagger \\
40 \mathrm{~cm}\end{array}$ & $\begin{array}{c}11.8 \mathrm{in} \dagger \\
30 \mathrm{~cm}\end{array}$ & $\begin{array}{c}7.9 \mathrm{in} \dagger \\
20 \mathrm{~cm}\end{array}$ & $3.0 \mathrm{~kg} \dagger$ & $0.75 \mathrm{~kg} \dagger$ \\
\hline
\end{tabular}

Table 2.1. Micromagic Systems hexapod robot specifications (Berns 2005; Denton 2008).

\section{* estimated values from images †approximate values since i.C. Hexapod is based on the Hexapod V4 chassis}

All of the hexapod robots are power autonomous with on-board batteries. Each of the first two models use an off-board computer for calculating joint movements and an on-board servo motor controller (similar to BILL-Ant-p), while Hexapod V3 had the option to use an on-board p.Brain controller (a PIC-based microcontroller developed by Matt Denton) for joint movement calculations. Later models used the p.Brain controller for on-board joint control autonomy, but maintained a wireless connection to a host computer for high-level commands, and image processing in the case of the i.C. Hexapod. 


\subsubsection{A-pod}

Kåre Halvorsen (a.k.a. Zenta) is a robot hobbyist from Norway who created the A-pod hexapod in 2009. It is based on the same control scheme of the Phoenix hexapod, designed by Jeroen Janssen (a.k.a. Xan) and currently sold as a kit by Lynxmotion, which is discussed in Section 2.1.6. The A-pod is a hexapod with six 3-DoF legs, a 3-DoF neck, a 1-DoF pair of mandibles (using one motor for each mandible, but only one control signal), and a 2-DoF abdomen for a total of 24 DoF and 25 actuators (Fig. 2.9) (Ackerman 2010; Zenta 2009a; Zenta 2009b).

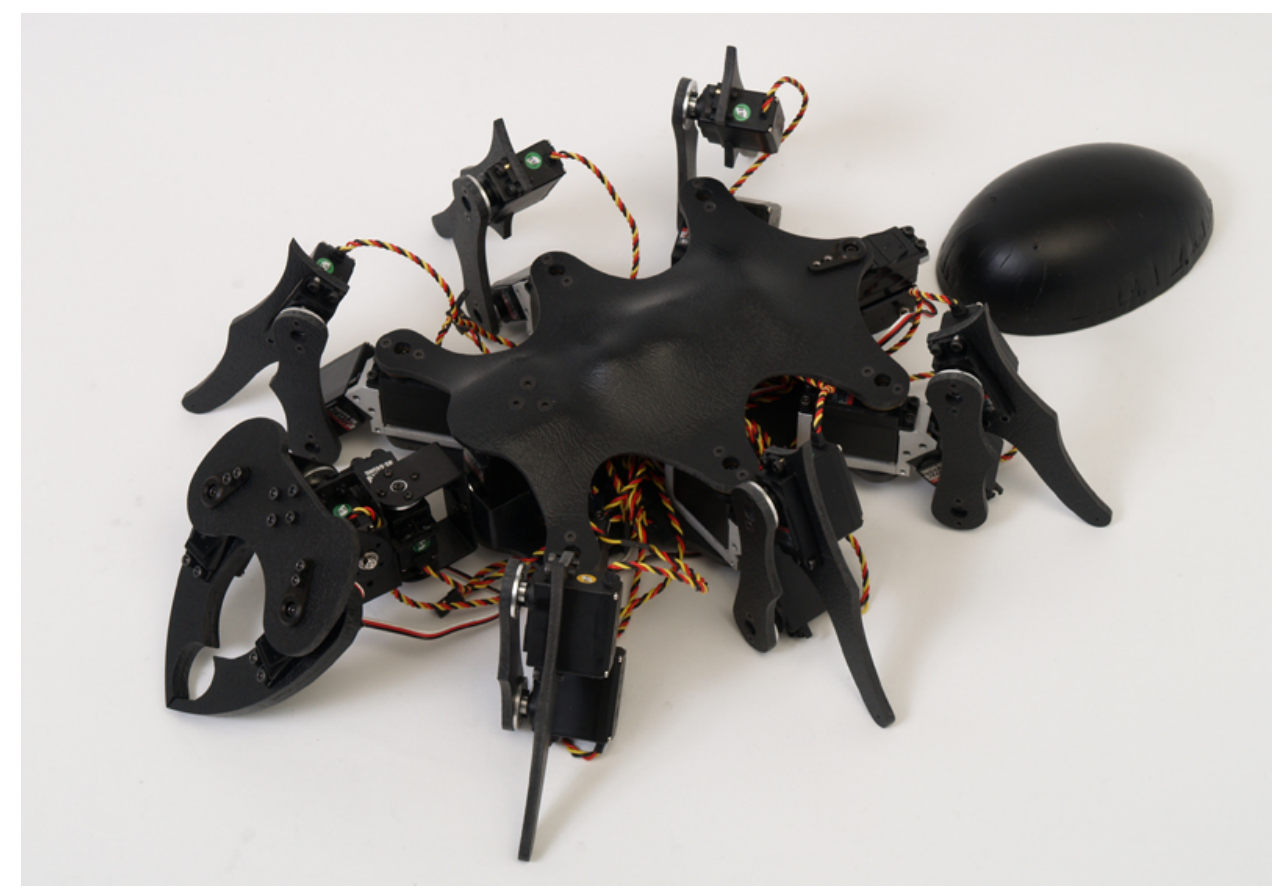

Figure 2.9. A-pod hexapod designed by Kåre Halvorsen (a.k.a. Zenta)

(Ackerman 2010; Zenta 2009a; Zenta 2009b).

Most of the A-pod is constructed from 0.20in (5.0mm)-thick ABS textured plastic. Custom-machined aluminum parts are used to couple the coxa and femur motors, responsible for protraction/retraction and levation/depression, 
respectively (Note: Zenta's naming convention for the leg joints differs from BILL-Ant-a). Servo Erector Set aluminum brackets from Lynxmotion (Lynxmotion, Inc., Pekin, IL, USA) are used to couple the degrees-of-freedom within the neck and within the abdomen. Hitec HS-645MG high torque analog hobby servo motors are used throughout the robot for actuation, and are mounted such that they directly drive their connected elements. Two digital Towardpro MG996R servos are used to actuate the mandible pieces.

On-board control electronics are located in the abdomen and consist of a Lynxmotion Bot Board II carrier (BB2) holding a Basic Micro BasicATOM Pro28 (BAP28) (Basic Micro, Murrieta, CA, USA), and a Lynxmotion SSC-32 Servo Controller. The BAP28, a small and powerful 32-bit microcontroller, responds to user inputs from a custom-built wireless remote that employs an Xbee $2.4 \mathrm{GHz}$ transceiver. User commands are interpreted and converted to motor positions by the BAP28 performing linear transformations and interpolations of inverse kinematics (IK) functions. Trigonometric calculations are not performed, but rather pre-solved cosine and sine results are pulled from look-up tables stored in memory. The motor positions are then sent to the SSC-32 controller to be converted into hobby servo PWM signals. The 25 hobby servos located throughout the robot are connected to the SSC-32.

Power is provided by a single two-cell Li-Po battery $(7.4 \mathrm{vdc}, 5100 \mathrm{mAh})$ located in the base of the thorax, which is then converted down to 6vdc by a Turnigy Redundant Dual 8A UBEC (ultimate battery elimination circuit) (Hobby King / 
HexTronix Ltd., Hong Kong). The UBEC is used to supply both controller and motor power.

Combinations of button and joystick actions allow the robot to fully alter its body posture (roll, pitch, and yaw) about several selectable anchor points (body center, neck, head, or abdomen joint). Walking is non-holonomic and is directed by two, two-axis joysticks on the remote transmitter. The joysticks control two-axis translation (combinations of forward/reverse and lateral left/lateral right) and oneaxis rotation (left/right). The signals from the two joysticks are combined into a composite behavior that allows for both translation and rotation. A series of userselectable gaits, such as ripple (wave) and alternating tripod, determine the walking style.

Much like the Micromagic Systems robots, the A-pod has smooth, fluid movements, mostly due to its linear interpolation inverse kinematics method. Command of the robot is done by a user operating a wireless controller, as there are no sensors or autonomous operation designed into the unit (with the exception of force sensing in the mandibles to limit mandible torque), the high level of agility and uncannily 'natural' articulation is commendable.

In its nominal stance, photographs of the A-pod estimated it to be $19.5 \mathrm{in}(50 \mathrm{~cm})$ long, 13.5in (34cm) wide, and 7.5in (19cm) tall (Zenta 2009a). 


\subsubsection{Additional 18-DoF Hexapod Robots}

Many other motor-actuated 18-DoF hexapod robots have been built over the years, some of which are listed in Table 2.2 along with the previously described robots. Table 2.3 compares general specifications of the robots mentioned above with the BILL-Ant-a robot.

\begin{tabular}{|c|c|c|c|}
\hline Robot & Head Professor & Institute & $\begin{array}{c}\text { Selected } \\
\text { References }\end{array}$ \\
\hline $\begin{array}{l}\text { AMOS- } \\
\text { WD06 }\end{array}$ & Dr. Hubert Roth & Universität Siegen & $\begin{array}{l}\text { Manoonpong et al. } \\
2007\end{array}$ \\
\hline A-pod & $\begin{array}{l}\text { Kåre Halvorsen } \\
\text { (a.k.a. Zenta) }\end{array}$ & -- & $\begin{array}{l}\text { Ackerman 2010; } \\
\text { Zenta 2009a; } \\
\text { Zenta 2009b }\end{array}$ \\
\hline Attila II & $\begin{array}{l}\text { Dr. Rodney } \\
\text { Brooks }\end{array}$ & MIT & $\begin{array}{l}\text { Angle 1991; } \\
\text { Ferrell } 1993\end{array}$ \\
\hline BILL-Ant-p & Dr. Roger Quinn & $\begin{array}{l}\text { Case Western } \\
\text { Reserve University, } \\
\text { Biorobotics Lab }\end{array}$ & Lewinger 2005 \\
\hline $\begin{array}{l}\text { LAURON, } \\
\text { LAURON II, } \\
\text { LAURON III }\end{array}$ & Dr. Karsten Berns & $\begin{array}{l}\text { Forschungszentrum } \\
\text { Informatik }\end{array}$ & $\begin{array}{l}\text { Gaßmann et al. } \\
1991\end{array}$ \\
\hline $\begin{array}{l}\text { Phoenix } \\
\text { 3DOF } \\
\text { Hexapod, } \\
\text { BH3 }\end{array}$ & -- & Lynxmotion, Inc. & $\begin{array}{l}\text { Lynxmotion } \\
\text { Phoenix 2008; } \\
\text { Lynxmotion BH3 } \\
2008\end{array}$ \\
\hline Robot II & Dr. Roger Quinn & $\begin{array}{l}\text { Case Western } \\
\text { Reserve University, } \\
\text { Biorobotics Lab }\end{array}$ & $\begin{array}{l}\text { Espenschied and } \\
\text { Quinn 1994; } \\
\text { Espenschied et al. } \\
1995 \\
\end{array}$ \\
\hline $\begin{array}{l}\text { Tarry I, } \\
\text { Tarry II }\end{array}$ & Dr. Martin Frik & $\begin{array}{l}\text { University of } \\
\text { Duisberg }\end{array}$ & Buschmann 2000a \\
\hline $\begin{array}{l}\text { TUM } \\
\text { Walking } \\
\text { Machine }\end{array}$ & $\begin{array}{l}\text { Dr. Friedrich } \\
\text { Pfeiffer }\end{array}$ & $\begin{array}{l}\text { Technische } \\
\text { Universität } \\
\text { München }\end{array}$ & Pfeiffer et al. 1994 \\
\hline xPod V4 & Matt Denton & $\begin{array}{l}\text { Micromagic } \\
\text { Systems }\end{array}$ & $\begin{array}{l}\text { Micromagic } \\
\text { Systems } 2008 \\
\end{array}$ \\
\hline
\end{tabular}

Table 2.2. 3-DoF per leg hexapod robot general information chart. 


\begin{tabular}{|c|c|c|c|c|c|c|c|c|c|c|}
\hline $\begin{array}{l}\overrightarrow{0} \\
\ddot{\theta}\end{array}$ & 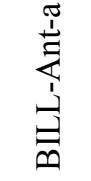 & 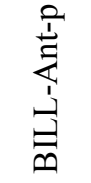 & 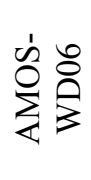 & $\underset{E}{E}$ & $\frac{⿱}{>}$ & $\begin{array}{l}7 \\
0 \\
\frac{0}{1} \\
\frac{1}{4}\end{array}$ & 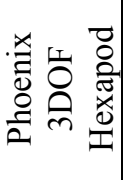 & 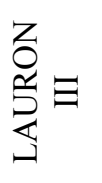 & 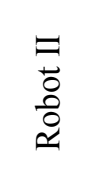 & 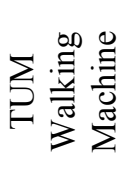 \\
\hline Length & $\begin{array}{r}0.33 \\
\mathrm{~m}\end{array}$ & $\begin{array}{r}0.33 \\
\mathrm{~m}\end{array}$ & $\begin{array}{r}0.40 \\
\mathrm{~m}\end{array}$ & $\begin{array}{r}0.50 \\
\mathrm{~m}\end{array}$ & $\begin{array}{l}0.4 \\
0 \mathrm{~m}\end{array}$ & $\begin{array}{r}0.50 \\
\mathrm{~m}^{*}\end{array}$ & $\begin{array}{r}0.43 \\
\mathrm{~m}\end{array}$ & $\begin{array}{r}0.50 \\
\mathrm{~m}\end{array}$ & $\begin{array}{r}0.50 \\
\mathrm{~m}\end{array}$ & $\begin{array}{r}0.80 \\
\mathrm{~m}\end{array}$ \\
\hline Width & $\begin{array}{r}0.33 \\
\mathrm{~m}\end{array}$ & $\begin{array}{r}0.33 \\
\mathrm{~m}\end{array}$ & $\begin{array}{r}0.12 \\
\mathrm{~m}\end{array}$ & $\begin{array}{r}0.20 \\
\mathrm{~m}\end{array}$ & $\begin{array}{r}0.20 \\
\mathrm{~m}\end{array}$ & $\begin{array}{r}0.34 \\
\mathrm{~m}^{*}\end{array}$ & $\begin{array}{r}0.37 \\
\mathrm{~m}\end{array}$ & $\begin{array}{r}0.30 \\
\mathrm{~m}\end{array}$ & $\begin{array}{r}0.25 \\
\mathrm{~m}\end{array}$ & $\begin{array}{r}0.40 \\
\mathrm{~m}\end{array}$ \\
\hline Height & $\begin{array}{r}0.15 \\
\mathrm{~m}\end{array}$ & $\begin{array}{r}0.15 \\
\mathrm{~m}\end{array}$ & $\begin{array}{r}0.30 \\
\mathrm{~m}\end{array}$ & $\begin{array}{r}0.40 \\
\mathrm{~m}\end{array}$ & $\begin{array}{r}0.30 \\
\mathrm{~m}\end{array}$ & $\begin{array}{r}0.19 \\
\mathrm{~m}^{*}\end{array}$ & $\begin{array}{r}0.13 \\
\mathrm{~m}\end{array}$ & $\begin{array}{r}0.80 \\
\mathrm{~m}\end{array}$ & $\begin{array}{r}0.50 \\
\mathrm{~m}\end{array}$ & $\begin{array}{r}1.0 \\
\mathrm{~m}\end{array}$ \\
\hline $\begin{array}{c}\text { Max. } \\
\text { Speed }\end{array}$ & $\begin{array}{r}0.03 \\
\mathrm{~m} / \mathrm{s}\end{array}$ & $\begin{array}{r}0.004 \\
\mathrm{~m} / \mathrm{s}\end{array}$ & $\begin{array}{r}0.10 \\
\mathrm{~m} / \mathrm{s}\end{array}$ & $\begin{array}{r}0.20 \\
\mathrm{~m} / \mathrm{s}\end{array}$ & $\begin{array}{r}0.30 \\
\mathrm{~m} / \mathrm{s}\end{array}$ & $\begin{array}{c}0.30 \\
\mathrm{~m} / \mathrm{s}^{*}\end{array}$ & $\begin{array}{r}0.25 \\
\mathrm{~m} / \mathrm{s}\end{array}$ & $\begin{array}{r}0.40 \\
\mathrm{~m} / \mathrm{s}\end{array}$ & $\begin{array}{r}0.14 \\
\mathrm{~m} / \mathrm{s}\end{array}$ & $\begin{array}{r}0.30 \\
\mathrm{~m} / \mathrm{s}\end{array}$ \\
\hline Weight & $\begin{array}{r}2.85 \\
\mathrm{~kg}\end{array}$ & $\begin{array}{l}2.3 \\
\mathrm{~kg}\end{array}$ & $\begin{array}{r}4.2 \\
\mathrm{~kg}\end{array}$ & $\begin{array}{r}2.9 \\
\mathrm{~kg}\end{array}$ & $\begin{array}{r}3.0 \\
\mathrm{~kg}\end{array}$ & $\begin{array}{r}2.0 \\
\mathrm{~kg} *\end{array}$ & $\begin{array}{l}1.6 \\
\mathrm{~kg}\end{array}$ & $\begin{array}{l}18 \\
\mathrm{~kg}\end{array}$ & -- & $\begin{array}{l}23 \\
\mathrm{~kg}\end{array}$ \\
\hline Payload & $\begin{array}{r}8.6 \\
\mathrm{~kg}\end{array}$ & $\begin{array}{r}8.6 \\
\mathrm{~kg}\end{array}$ & $\begin{array}{r}1.0 \\
\mathrm{~kg}\end{array}$ & $\begin{array}{r}2.9 \\
\mathrm{~kg}\end{array}$ & $\begin{array}{r}0.75 \\
\mathrm{~kg}\end{array}$ & -- & -- & $\begin{array}{r}10.0 \\
\mathrm{~kg}\end{array}$ & -- & $\begin{array}{c}5.0 \\
\mathrm{~kg}\end{array}$ \\
\hline Legs & 6 & 6 & 6 & 6 & 6 & 6 & 6 & 6 & 6 & 6 \\
\hline $\begin{array}{c}\text { Active } \\
\text { Degrees }\end{array}$ & 19 & 22 & 21 & 18 & 18 & 25 & 18 & 18 & 18 & 18 \\
\hline $\begin{array}{l}\text { Passive } \\
\text { Degrees }\end{array}$ & 12 & 10 & 6 & 0 & 6 & 1 & 0 & 0 & 6 & 0 \\
\hline Actuators & $\begin{array}{r}\text { DC } \\
\text { Servos }\end{array}$ & $\begin{array}{r}\text { DC } \\
\text { Servos }\end{array}$ & $\begin{array}{r}\text { DC } \\
\text { Servos }\end{array}$ & $\begin{array}{r}\text { DC } \\
\text { Servos }\end{array}$ & $\begin{array}{r}\text { DC } \\
\text { Servos }\end{array}$ & $\begin{array}{r}\text { DC } \\
\text { Servos }\end{array}$ & $\begin{array}{r}\text { DC } \\
\text { Servos }\end{array}$ & $\begin{array}{r}\text { DC } \\
\text { Servos }\end{array}$ & $\begin{array}{r}\text { DC } \\
\text { Motors }\end{array}$ & $\begin{array}{r}\text { DC } \\
\text { Motors }\end{array}$ \\
\hline $\begin{array}{l}\text { Power } \\
\text { Supply }\end{array}$ & $6 \mathrm{~V}$ & $6 \mathrm{~V}$ & $\begin{array}{l}6 \mathrm{~V}, \\
9 \mathrm{~V}\end{array}$ & $12 \mathrm{~V}$ & $4.8 \mathrm{~V}$ & $6 \mathrm{~V}$ & $6 \mathrm{~V}$ & $12 \mathrm{~V}$ & $12 \mathrm{~V}$ & -- \\
\hline Control & $\begin{array}{l}\text { on- } \\
\text { board }\end{array}$ & $\begin{array}{r}\text { off- } \\
\text { board }\end{array}$ & $\begin{array}{r}\text { on- } \\
\text { board }\end{array}$ & $\begin{array}{r}\text { off- } \\
\text { board }\end{array}$ & $\begin{array}{r}\text { on- } \\
\text { board }\end{array}$ & $\begin{array}{r}\text { on- } \\
\text { /off- } \\
\text { board }\end{array}$ & $\begin{array}{r}\text { on- } \\
\text { board }\end{array}$ & $\begin{array}{r}\text { on- } \\
\text { board }\end{array}$ & $\begin{array}{r}\text { off- } \\
\text { board }\end{array}$ & $\begin{array}{r}\text { on- } \\
\text { board }\end{array}$ \\
\hline Power & $\begin{array}{r}\text { on- } \\
\text { board }\end{array}$ & $\begin{array}{r}\text { on- } \\
\text { board }\end{array}$ & $\begin{array}{r}\text { on- } \\
\text { /off- } \\
\text { board }\end{array}$ & $\begin{array}{r}\text { off- } \\
\text { board }\end{array}$ & $\begin{array}{r}\text { on- } \\
\text { board }\end{array}$ & $\begin{array}{r}\text { on- } \\
\text { board }\end{array}$ & $\begin{array}{r}\text { on- } \\
\text { board }\end{array}$ & $\begin{array}{r}\text { on- } \\
\text { board }\end{array}$ & $\begin{array}{r}\text { off- } \\
\text { board }\end{array}$ & $\begin{array}{r}\text { off- } \\
\text { board }\end{array}$ \\
\hline
\end{tabular}

Table 2.3. Selected 3-DoF per leg hexapod robot specifications comparison chart (Berns 2005, Zenta, 2009a, Lynxmotion Phoenix 2008).

* Estimated values 


\subsection{Hexapod Robot Control Schemes}

There are a variety of control methodologies for actuating joints and coordinating movements in legged robots. These range from pure engineering approaches that use mechanical constraints and mathematical methods of control, to animal and insect-inspired methods that mimic (to varying degrees) the motions, behaviors, and control schemes found in nature.

\subsubsection{Leg Control Methods}

This section describes several methods for coordinating the actions of the joints within a single leg (intra-leg) to generate stepping motions. These actions allow the legs to support the body and their payload; propel the body in a desired direction; and dictate the posture assumed by the robot.

The following sections briefly explain different methods that progress from engineering-based toward nature-inspired means of controlling leg stepping movements.

\subsubsection{Pure Mechanical Systems}

One way of controlling leg stepping actions is through mechanical coupling. This forms a pre-described series of relative joint movements that, in turn, enforce a set foot path (Boggess et al. 2004; Wei et al. 2004). The relative joint movements are enforced by a mechanical linkage, such as connected segments (Fig. 2.10), gears, or chain and sprocket systems. 
A benefit to this method is its simplicity in that it requires no computational effort to generate stepping actions. This is desirable for systems that either do not have, or for which it is undesirable to include a computer or microcontroller in order control leg movements. Because of its level of simplicity, many small-scale walking toys also use this means of stepping.

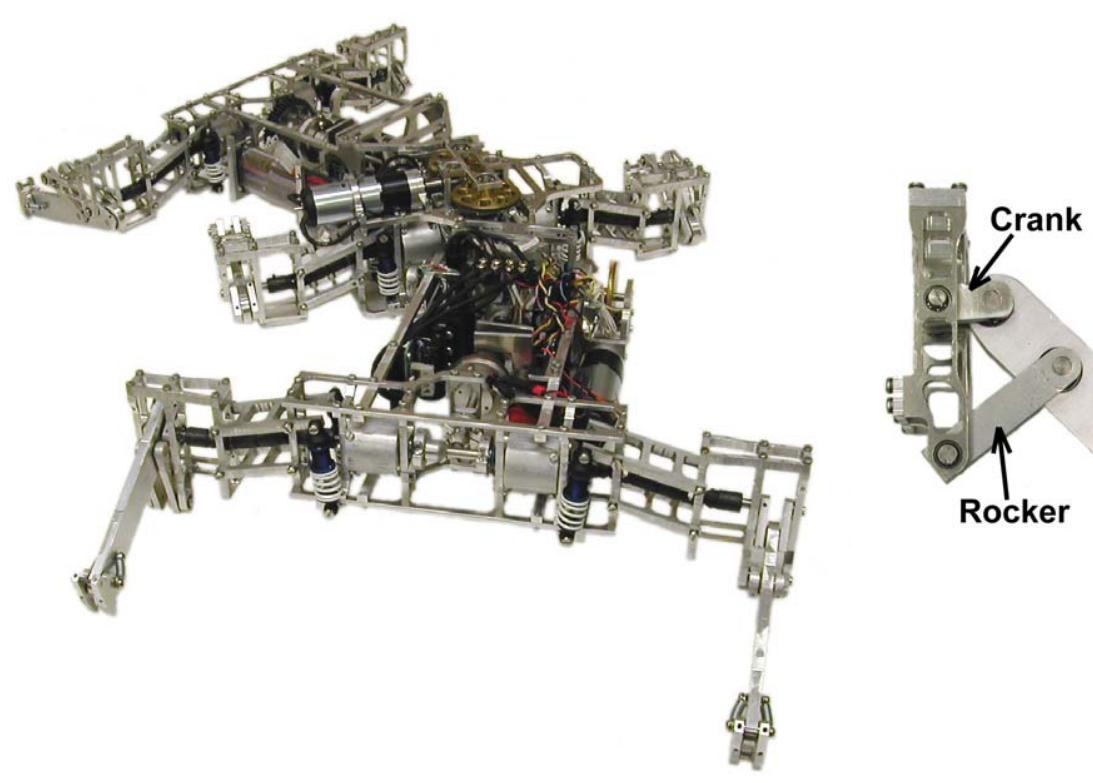

Figure 2.10. MechaRoach II (left), developed by Terence Wei, uses a fourbar mechanism (right) to mechanically determine the foot path (Wei et al. 2004)

Some robots, such as Whegs ${ }^{\mathrm{TM}}$ II (Allen et al. 2003), use remote control, or have been retrofitted with microcontrollers (Lewinger, Harley et al. 2005a; Lewinger, Watson, and Quinn 2006) to provide tele-operation or a level of autonomy by controlling the heading based on operator or sensory input. This additional layer of control does not change the shape of the mechanically-dictated foot path. It does, however, provide the execution of high-level actions, such as navigation, without the need of specifying low-level joint and foot path control. 


\subsubsection{Software-Implemented Fixed Step Path}

For robots using multiple, actuated joints per leg, electronic control is required to generate stepping actions. This does not, however, need to be a complicated nor robust method of joint control.

For some small-scale and simplified control scheme robots, such as the Lynxmotion hexapods (Lynxmotion BH3 2008; Lynxmotion Phoenix 2008), a microcontroller is used to move the leg joints through a pre-programmed series of motions. While these motions could be modulated by sensory information, the basic level of software uses pre-defined routines.

This method relies on a series of joint position sets being arranged in a predefined sequence. This yields a pre-defined set of leg poses, which represent a single stepping action. The software, such as in Fig. 2.11 for example, reads each set of joint position values stored in memory during each computation time increment and then commands each of the joints to that set of stored values. At each time increment, the next set of joint positions is read and then commanded. After the last set of values is used to form a pose, the list of sets is repeated from the first pose in order to form continuous stepping motions. These motions require very little computational complexity, but can require large amounts of electronic memory, depending on the number of distinct poses in a single stepping motion. 


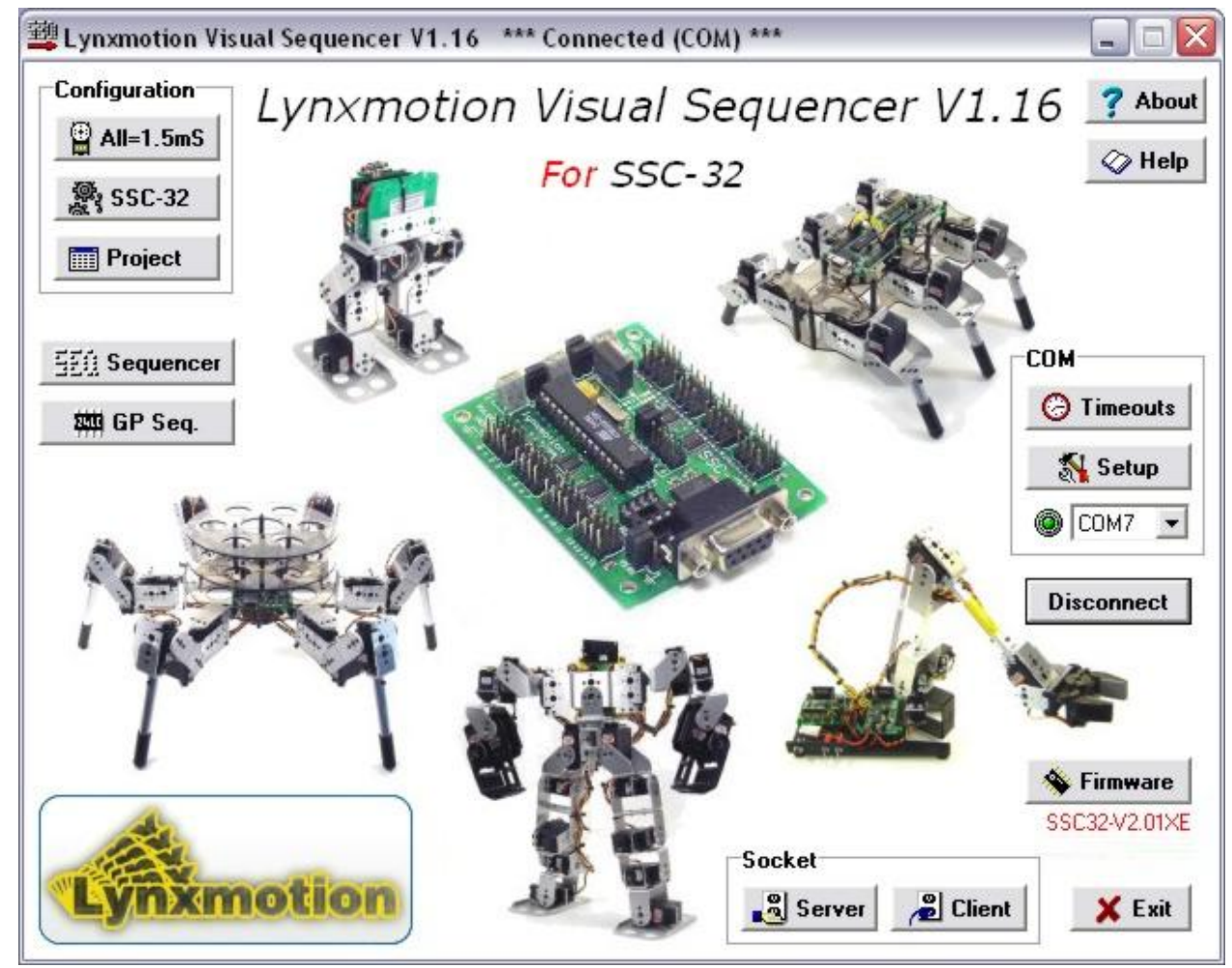

Figure 2.11. Lynxmotion, Inc. Visual Sequencer software.

This software package allows designers to define and sequence through a series of motions for their robot. These may be for generating stepping movements or other actions such as postures or specialized poses. (C) Lynxmotion, Inc.

To save memory space, some or all of the legs can use the same set of poses. Then, in order to create various gaits (discussed further in Section 2.2.2.2 and Section 2.2.2.3), relative leg phases can be determined by offsetting which pose in the series of sets is the initial pose for each leg. For example, to generate an alternating tripod gait, the front and rear legs on one side and the middle leg on the opposite site could begin with pose \#1 and proceed through the list of poses, say six in total (Fig. 2.12). The remaining three legs would then begin at pose \#4 of the same set, which would place those legs $180^{\circ}$ out-of-phase with the first legs. By repeating the list from the beginning after the last pose, each set of legs 
uses the complete set of six poses $\# 1-\# 6$ for the first tripod and $\# 4-6$ then $\# 1-$ \#3 for the second tripod).

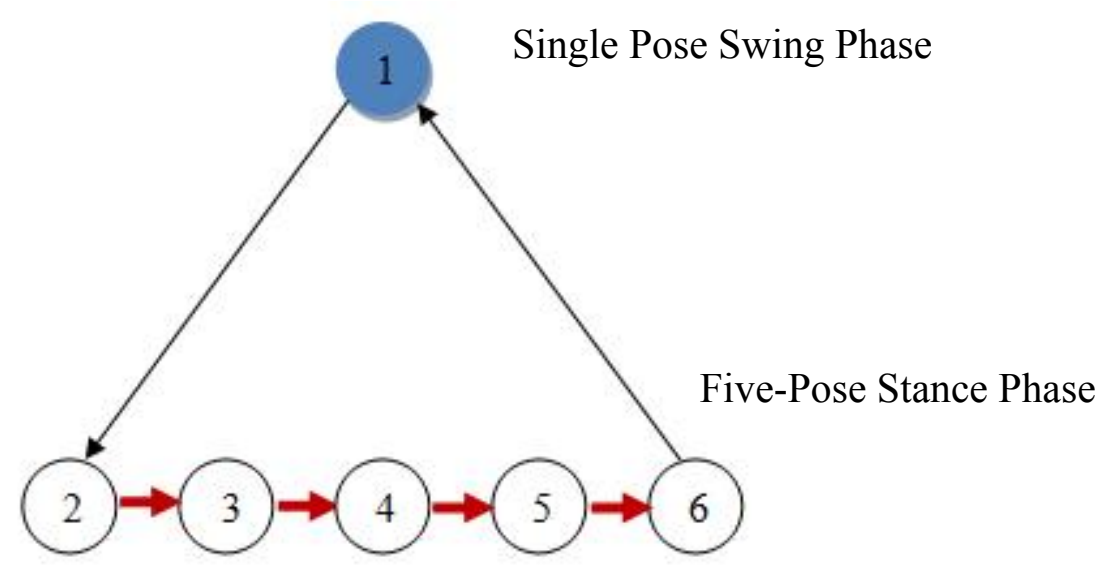

Figure 2.12. Six-pose stepping pattern option for the BlackWido hexapod.

This stepping pattern utilizes six distinct poses; one of which is used for the swing phase, and the remaining five poses constitute the stance phase. Cycling from one pose, through the complete set and back to the original pose constitutes as single step. (Xan 2009)

Unlike the pure mechanical implementation, this method allows the programmer to adjust the stepping styles by changing software parameters (instead of changing the physical system), which is an attractive prospect for quickly and easily dealing with varying environments, changes in desired stepping behaviors, or experimenting with various stepping motions without the need to manufacture or purchase new parts.

\subsubsection{Inverse Kinematics (IK)}

This numerical engineering method calculates the joint angles necessary to have the foot located at a desired point in the workspace of the limb. This is somewhat similar to the previous method, but the commands are a series of foot locations or 
a foot path equation instead of a series of joint positions. The joint positions required to achieve the desired foot location are then calculated dynamically by a series of equations. This method is useful when the designer needs to change the foot path due to heading changes or sensory inputs because it doesn't require multiple sets of joint poses for each possible, and often unforeseeable, foot path. Developers using a computer or powerful microcontrollers to determine joint movements can employ this method of deciding what joint angles to generate for a desired foot position or path of movement.

While this is a computationally-intensive method of generating stepping motions due to its use of trigonometric functions, some mathematical results can be precalculated and stored in look-up tables within memory, (e.g. the values of $\sin (x)$ for angles between $0 \mathrm{deg}$ and $180 \mathrm{deg}$ in $0.5 \mathrm{deg}$ increments). This greatly improves the speed of determining the IK calculations by reducing trigonometric equations to arithmetic ones.

BILL-Ant-p (Lewinger 2005; Lewinger, Branicky, and Quinn 2005), with an offboard computer, and Lauron III (Gaßmann et al. 2001), with on-board processing, each use trigonometric inverse kinematics to dynamically calculate the joint angles required to follow a parabolic foot path when legs are in swing phase, and straight-line paths when legs are in stance phase (Fig. 2.13, left). The foot path for a complete step is first divided into small segments, based on how far along the path a foot should move during a particular computation cycle. Each point along the segmented path represents a desired foot position in 3-D space. Then, 
inverse kinematics equations are used to calculate the joint angles required to reach that foot position (Fig. 2.13, right).

$$
\begin{aligned}
& \text { Distance from CTr Joint to foot: } \\
& r=\sqrt{\left(x-x_{0}\right)^{2}+\left(y-y_{0}\right)^{2}+\left(z-z_{0}\right)^{2}} \\
& \cos ^{-1}\left(\left(x-x_{0}\right) / r\right) \\
& \cos ^{-1}\left(\left(\left(x-x_{0}\right)^{2}+\left(y-y_{0}\right)^{2}\right)^{\frac{1}{2}} / r\right)
\end{aligned}
$$

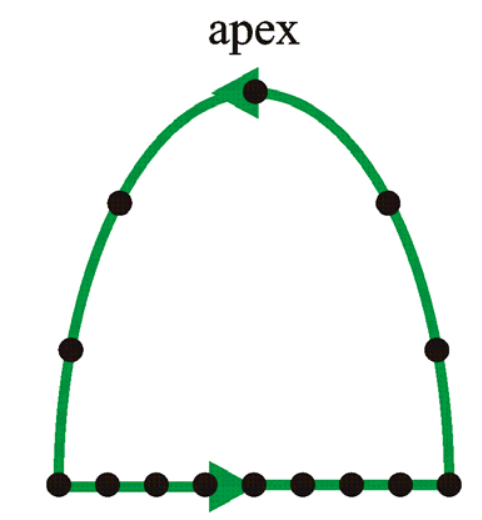

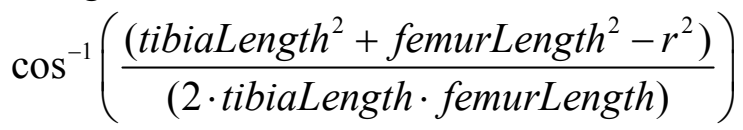

Figure 2.13. Parabolic swing and straight-line line stance foot trajectories for BILL-Ant-p stepping motion.

(left) This stepping motion has a parabolic path for the swing phase that is calculated based on the step length (distance between the AEP and PEP) and the desired step height (apex). The path is segmented into discrete points based on how far the foot should have travelled in a single calculation cycle. Inverse kinematics equations (right) are then used to determine the joint angles necessary to place the foot at each point along the path. (Lewinger 2005)

When it is desired for the robot to walk in a different direction, or at a different elevation because of differences in ground height for each of the legs, new foot paths can be determined. These new, on-the-fly foot paths are then segmented, as before, and IK is used to calculate the necessary joint angles. All of this occurs without the need to pre-program and store all of the possible leg poses in the work space, which could be memory capacity prohibitive. 


\subsubsection{Central Pattern Generators (CPGs) and Artificial Neural Networks (ANNs)}

A more computationally-efficient manner of generating leg motions to create stepping actions is to employ a central pattern generator (CPG). Manoonpong (2008) uses a CPG to create oscillatory outputs that are used as base timing signals (Fig. 2.14, upper left). The periodic output of the oscillator is then used to drive additional artificial neural network (ANN) elements that are responsible for controlling the duty cycle (speed of extension and flexion for a joint) and relative phase differences (timing of when each joint switches between extension and flexion as compared to other joints in the leg) for the joints. Setting the phase differences only determines when each joint is moving in one of its directions relative to other joints in the leg. However, through the mechanical coupling of the leg structure (i.e. the coxa, femur, and tibia that connects each of the joint actuators) a stepping pattern is created. The duty cycle, amplitude, and phase relationships of each joint-driving neuron output (e.g. neurons $\mathrm{H}_{13}$ and $\mathrm{H}_{14}$ in Fig. $2.14, b)$ are critical to coordinating the joint motions.

When the duty cycles of the joints are fixed, controlling the firing rate of the oscillator network (Fig. 2.14, upper left) determines the speed of leg movements; where increasing the firing rate increases the oscillation speed of the leg joints, which then increases the speed of the stepping motion. The converse is also true for decreasing the firing rate. By maintaining the relative phase differences of the joint-driving neuron outputs, the joints remain coordinated with one another to generate consistent stepping motions, independent of joint movement speeds. 
Making alterations in the relative phase differences (Fig. 2.14, b and c) will result in the generation of different stepping motions.
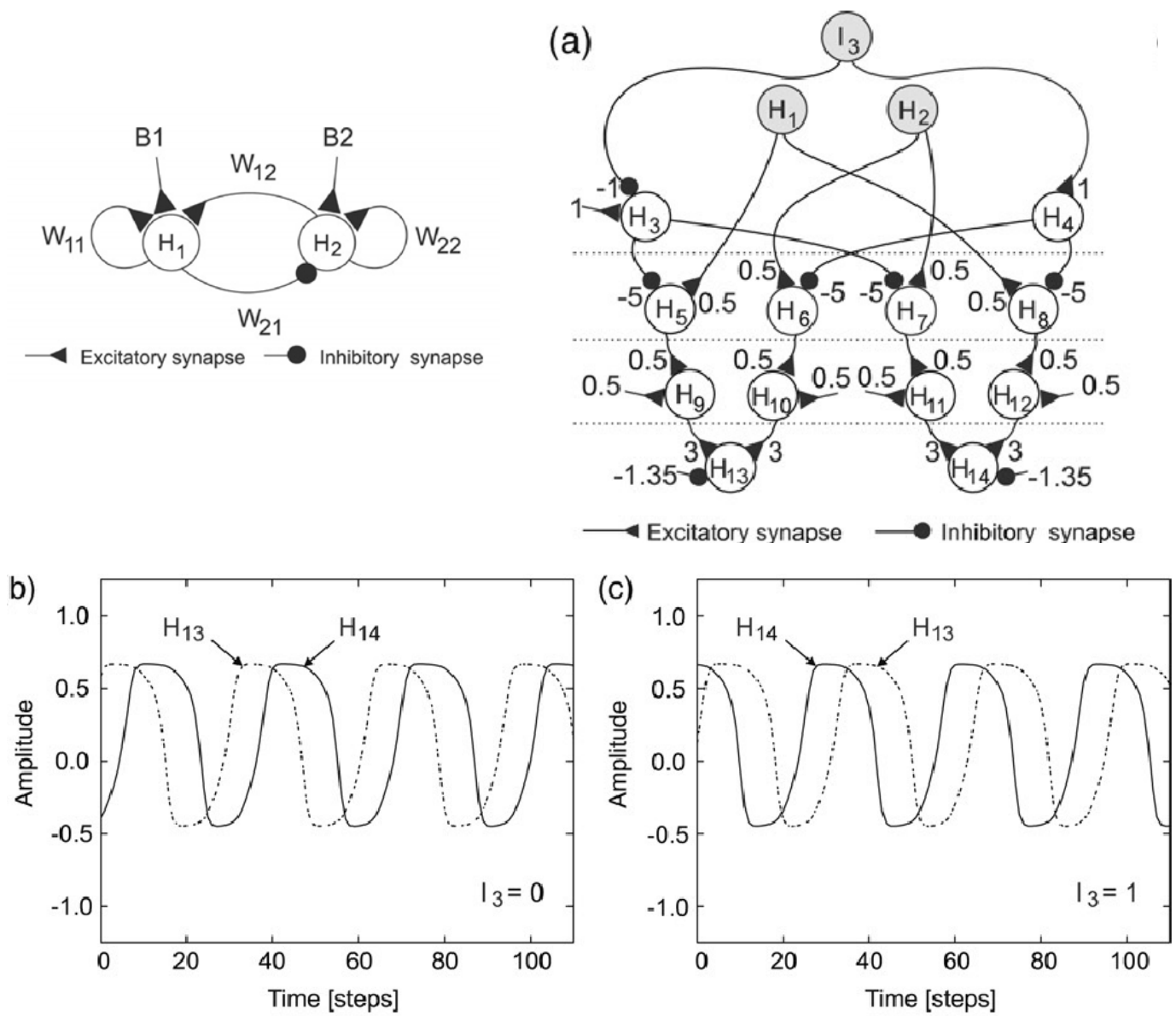

Figure 2.14. Leg movement central pattern generator for AMOS-WD06.

(upper left) The structure of the neural oscillator network that generates the base timing for the whole system, where $B_{1}=B_{2}=0.01$. (1) The phase switching network (PSN) responsible for setting the phase difference between the CTr and FTi joints. (b) When input $I_{3}$ (from (a)) is set to $0, H_{13}$ (FTi joint) leads $\mathrm{H}_{14}$ (CTr joint) and the robot walks toward the right. (c) When input $I_{3}=1$, the phase difference between CTr and FTi joint oscillations is reversed and the robot walks toward the left. The output of $\mathrm{H}_{14}$ is also used to drive an additional network (not shown) that is responsible for the movement of the ThC joints. (Manoonpong et al. 2008)

Another use for ANNs is for learning and following foot path trajectories, as is the case for Walknet (Schmitz et al. 2001; Schmitz 1998). This method employs 
a mesh of 33 neurons for swing phase movements (Swing Net) and a series of comparator and summation blocks to command stance phase movements (Stance Net). The special Target Net block is used for follow-the-leader behaviors, where feet are placed at the same spot as the feet of the next anterior leg. When operating, the joint velocities (which are the system outputs, not joint positions) are determined by simple arithmetic functions, rather than complex trigonometric IK equations.

\subsubsection{Leg Coordination Methods}

As with the coordination of individual joints within a single leg, there are a variety of methods for coordinating multiple legs (inter-leg control) to generate gaits. Orchestrated gaits allow the legs to move harmoniously through identifiable patterns that propel the robot in a stable, deliberate manner (Fig. 2.15).

Altering the gait changes the number of legs in contact with the ground at any given time, in which order they step, and the timing with which each leg begins its swing and stance phases relative to the others. As a result, changes in the gait affect how much support the legs provide to the body and any payload, and how much propulsive force the legs can exert in order to move the robot.

As more legs are in contact with the ground, the body will be better supported, more stable, and able to move with more force. Yet, having many legs on the 
a.

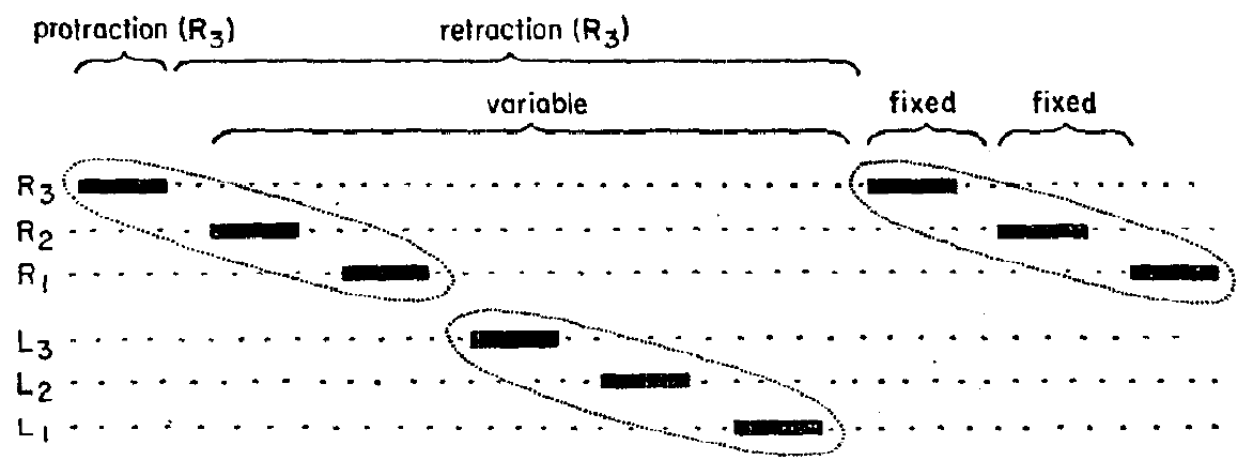

b.

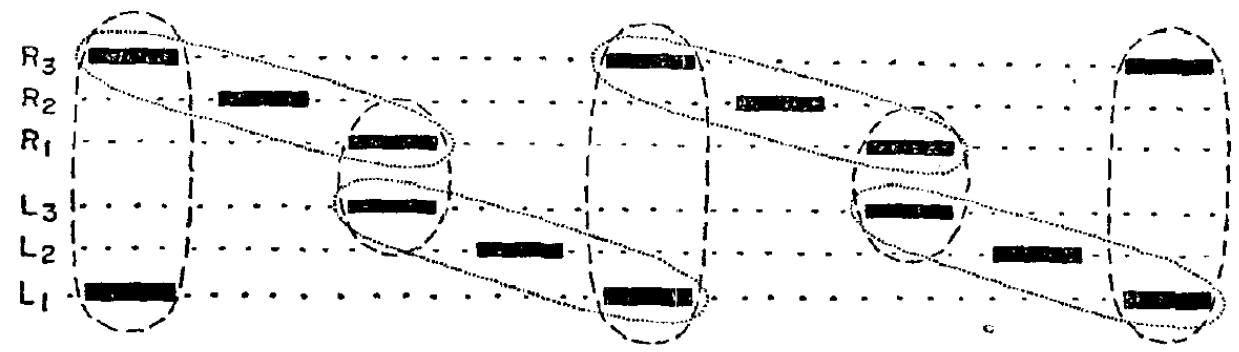

c.

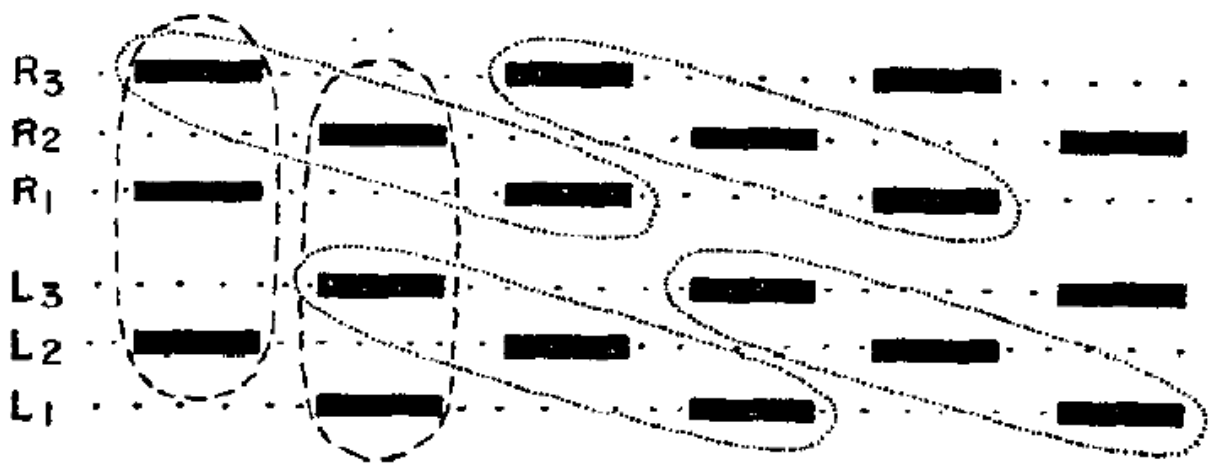

Figure 2.15. Gait patterns progressing from slow to fast walking.

(a) Slow wave (ripple) gait where only one leg is in its swing phase at a time and five legs are in their stance phase. (b) Medium speed tetrapod gait where one or two legs are in their swing phases simultaneously, leaving $4-5$

legs supporting and propelling the body. (c) Fast alternating tripod gait where a set of three legs cycle between swing and stance phases and are antiphase with the remaining three legs. (Wilson 1966)

ground simultaneously involves longer stance phases, implying that the robot will walk more slowly (Dean 1991b; Wilson 1966). Insects have been observed to use 
different gaits depending on the carried payload and walking speed (Dean 1991c). While walking slowly or with heavier loads, ripple or wave gaits are employed where $5-6$ legs are in contact with the ground at any time. When running, a rapid alternating tripod gait is used where three legs are in their swing phase simultaneously (Dean 1991b; Wilson 1966).

\subsubsection{Coupled Mechanical Systems}

The simplest, yet least robust method of gait generation, coupled mechanical systems use a fixed gait that is designed into the leg linkages. Typically, gears, a sprocket and chain, or tie rods couple the legs together with a single drive source.

This method is frequently found in small toys and simplified vehicles (Fig. 2.16).
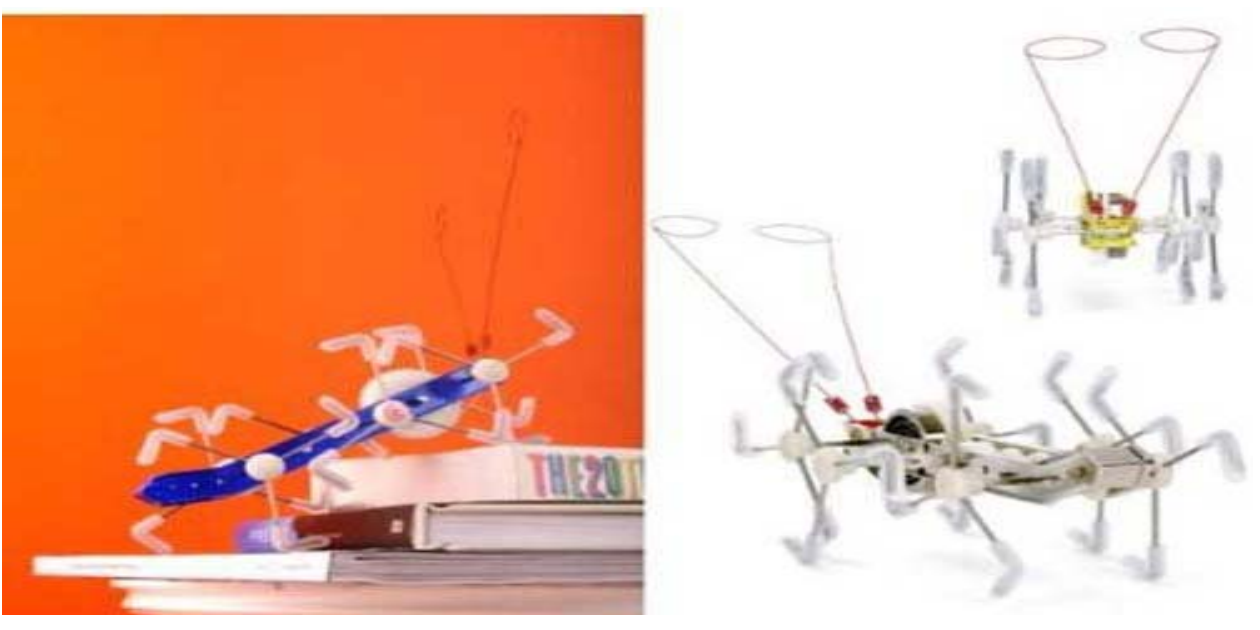

Figure 2.16. Wind-up toy hexapod (MxyKikker) with mechanically coupled legs (via gears) that walks in an alternating tripod. OKikkerland

Like the purely mechanical-based intra-leg joint coordination methods described in Section 2.2.1.1, coupled mechanical systems require no computation to generate the gait and frequently use an alternating tripod gait for hexapod 
vehicles, where the front and rear legs are in phase with the contralateral middle leg.

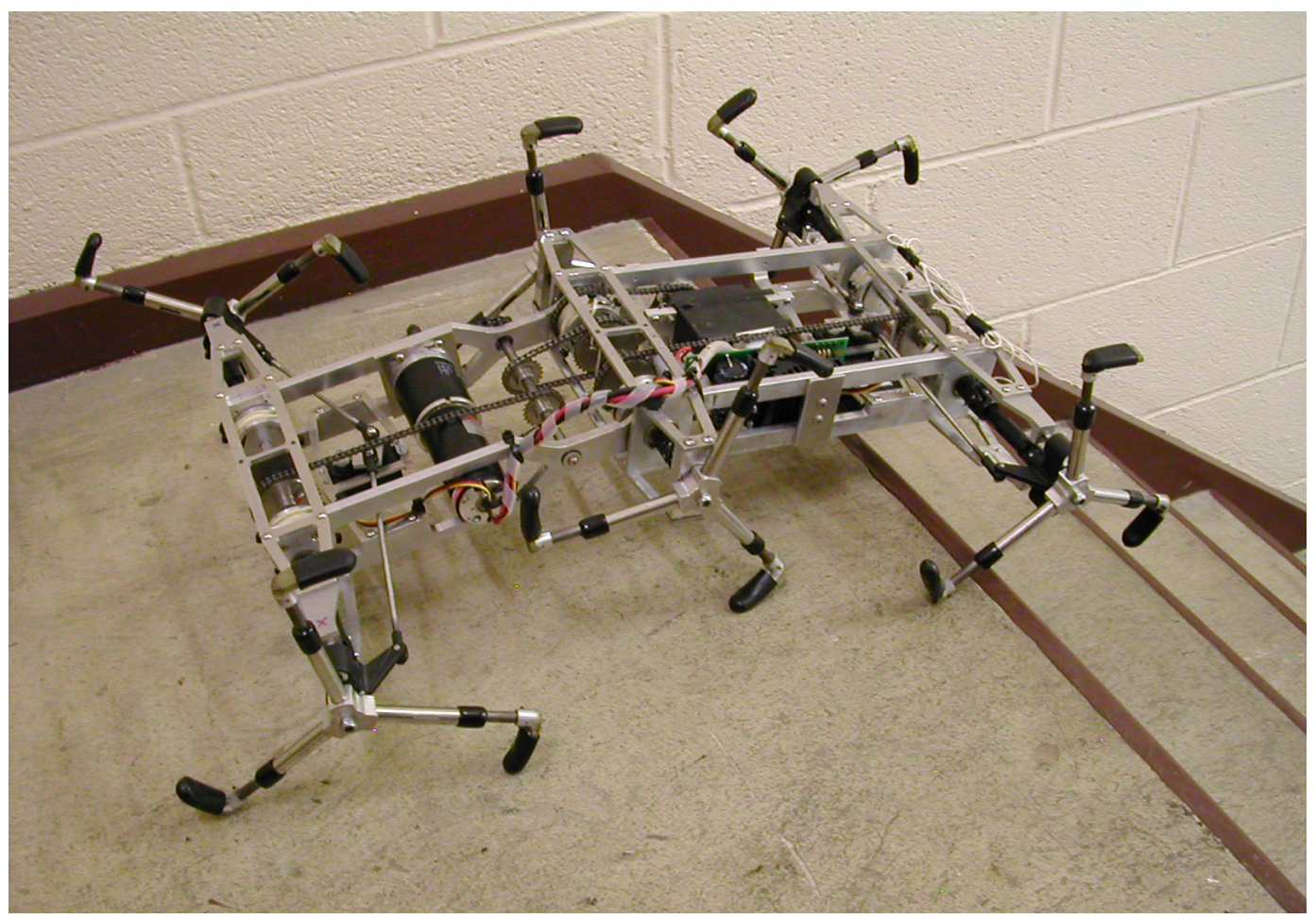

Figure 2.17. Whegs ${ }^{\mathrm{TM}}$ II robot

It employs a chain-and-sprocket drive system to actuate and maintain the gait of the wheel-legs. Torsional compliance devices located between the drive shafts and the wheel-leg hubs provide passive gait adaptation by allowing contralateral legs to come into phase with one another (Allen $e$ t al. 2003).

Mobile robots such as Whegs ${ }^{\mathrm{TM}}$ (Allen et al. 2003) vehicles are a variation of this type of system. Whegs ${ }^{\mathrm{TM}}$ vehicles maintain gait and relative foot position through a chain-and-sprocket drive system. Large-sized Whegs ${ }^{\mathrm{TM}}$ vehicles also have the ability to passively adjust their gait including allowing contralateral legs to come into phase with one another (Fig. 2.17). This is accomplished through spring-loaded torsional compliance devices located between the drive sprocket 
and output shaft for each wheel-leg. These devices let contralateral legs come completely into phase, thereby allowing a greater torque transfer to the substrate in order to lift the body. This gives each leg the ability to adjust its relative phase depending on the terrain, which is especially useful for climbing. The small Mini Whegs ${ }^{\mathrm{TM}}$ vehicles, with the exception of the first model, which also had torsional compliance units, maintain a fixed gait through a chain-and-sprocket system (Morrey et al. 2003).

\subsubsection{Fixed Programmed Gaits}

Robots with limited computational ability microcontrollers often have fixed, software-defined gaits. Like the freely available software that comes with the Lynxmotion hexapod robots (Lynxmotion Phoenix 2008; Lynxmotion BH3 2008), predefined software gaits are analogous to an electronic version of mechanically-coupled systems. While not robust to changes in the terrain, fixed gaits are simple to implement in software and require a relatively small amount of processing ability.

There are several means of implementing this leg coordination method. Typically, a single step for a prototypical leg is created as a series of poses, usually as a look-up table of indexed joint positions, or by a formula (See Section 2.2.1.2). Creating continuous stepping motions for a single leg is accomplished by sequentially executing all of the poses in the look-up table or commanding the joint positions according to the formula. By repeating the actions for a single step, multiple, identical stepping motions are performed. 
To create stable gaits, different legs begin the stepping process at different indices within the step cycle. In hexapod robots, a frequently used gait is the alternating tripod. This is also referred to simply as the tripod gait. This is where the front and middle legs on one side of the robot are in phase with middle leg of the opposite side. The remaining three legs are then in phase with one another, but anti-phase with the first tripod. In this gait all neighboring legs are 180 degrees out of phase with one another.

\subsubsection{Dynamically Selectable Gaits}

The next evolutionary step in gait generation is to have a series of fixed, programmed gaits that can be switched as desired. For example, a wave gait may be used at slow speeds where the improved support of five or six legs is desirable. As the speed increases, a quadruped gait (one or two legs are in swing simultaneously), or tripod gait (three legs in swing simultaneously) can be used.

This requires a similar amount of memory for the gait generation controller as the fixed software gait, and only slightly more computation ability. As with the fixed gait method, stepping motions are generated by having a single leg follow a cycle of poses or a formula to create a single step. Different gaits are formed by having each leg begin the step at a different part of the cycle. By dynamically changing where the start of the cycle is for each leg, different gaits are seen.

Discrete, selectable gait patterns are another benefit to the software-implemented fixed step path method of joint coordination (Section 2.2.1.2). When the number 
of poses for a single step is easily divisible by the number of legs, additional gaits can be formed by altering the starting pose uniquely for each of the legs. As an example, when having the same collection of six joint angle sets for a single stepping cycle, as described in the example from Section 2.2.1.2, legs could have the following starting poses: RR leg - pose \#1; LR leg - pose \#2; RM leg - pose \#3; LM leg - pose \#4; RF leg - pose \#5; and LF leg - pose \#6 (Fig. 2.12). Using these offsets in the starting poses would create a metachronal ripple (wave) gait while using the same set of poses from the alternating tripod gait. This method of gait selection is used with the Lynxmotion hexapods (Lynxmotion BH3 2008; Lynxmotion Phoenix 2008.

In this method each discrete gait is selected based on some command parameter, such as user discretion, or desired speed. By using speed as the gait deciding factor, threshold values can be set such that at low speeds a wave gait is employed, and at higher speeds quadruped and tripod gaits are used in turn. As a new gait is desired, one or more legs are chosen as reference legs and maintain their stepping cycles. The remaining legs then change to a pose at a different index within the step cycle based on the relative phasing with the reference legs as required by the new gait.

\subsubsection{Oscillators / Central Pattern Generators (CPGs)}

As with the leg joint control method described in Section 2.2.1.4, central pattern generators (CPGs) can also be used to create coordinated stepping actions of multiple legs for gait generation. Whereas in intra-leg joint control, the CPG was 
used to oscillate between extension and flexion of individual joints, for leg coordination a CPG is used to create the timing for stance and swing phases of different legs. The phase relationships when each leg transitions between the swing and stance phases dictate the performed gait and are set by the connection weights between the artificial neurons. This leg coordination method is typically integrated within the network responsible for generating the stepping motions (Beer et al. 1997; Barnes 1998; Bailey et al. 2000; Porcino 1990; Rietman and Hillis 2006) as seen in Fig. 2.18.

Figure 2.18 depicts the artificial neural network responsible for controlling walking for the 2-DoF per leg hexapod Robot I (Beer et al. 1997; Espenschied and Quinn 1994). For this network, each leg is controlled by a collection of six artificial neurons: $\mathrm{P}$ - a pacemaker neuron responsible for choosing the direction of the leg (forward or backward; FAS and BAS - neurons that sense when the leg has reached the forward-most or backward-most joint angle, respectively and are inputs that instruct $\mathrm{P}$ when to change leg direction; FT - the foot motor neuron responsible for levation and depression of the leg during swing and stance phases, respectively; FS and BS - motor neurons that control the speed of the leg when in swing and stance phase, respectively; and finally, $\mathrm{C}$ - the command neuron excites the BAS neurons to induce the stance phase for the legs. Periodically, the pacemaker neurons will fire and switch the leg state from stance phase to swing phase. 


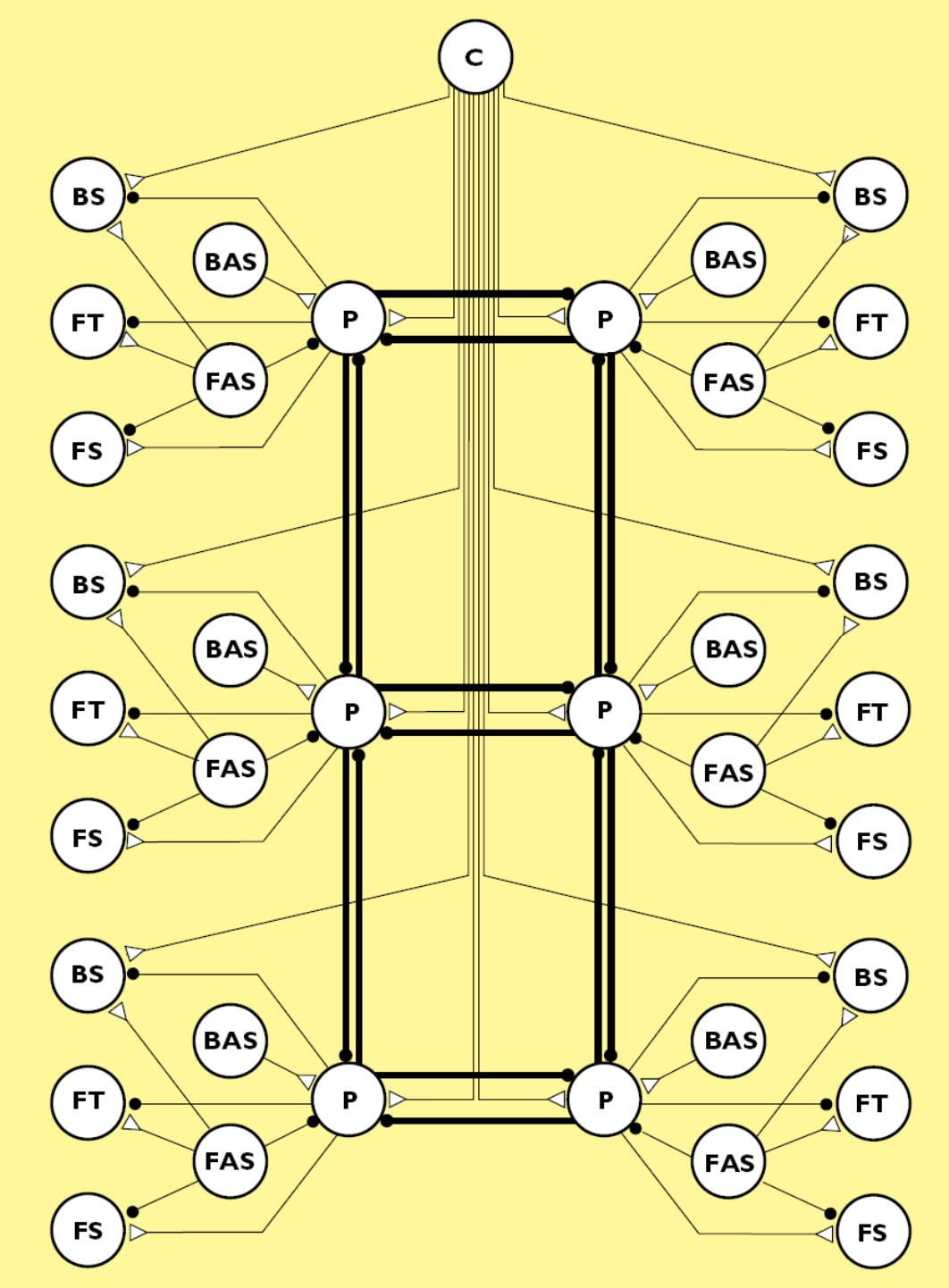

Figure 2.18. Leg control and coordination network for Robot I.

Schematic diagram of a distributed neural network for the control of locomotion. Excitatory connections are denoted by open triangles and inhibitory connections are denoted by filled circles. Abbreviations: C, command neuron; $P$, pacemaker neuron; FT, foot motor neuron; FS and BS, forward swing and backward swing motor neurons; FAS and BAS, forward and backward angle sensors (Beer et al. 1997)

The pacemaker neurons are also mutually inhibited for orthogonal neighbors. This would normally result in a persistent alternating tripod gait. However, differences in the nominal step lengths of the legs, where the middle legs have a 
slightly longer step than the front legs and the rear legs have a slightly longer step than the middle legs, allow the system to perform a metachronal wave (ripple) gait at slow walking speeds.

\subsubsection{Cruse's Rules for Leg Coordination}

In the mid 1970's Dr. Holk Cruse began research with stick insects to investigate nervous system feedback mechanisms that control leg movement (Cruse 1976; Cruse and Storrer 1977). By the mid 1980's Dr. Cruse and others (Cruse 1985; Cruse and Müller 1986) were observing leg movements and proposing possible mechanisms that cause contralateral and ipsilateral adjacent legs to influence one another. Figure 2.19 illustrates those hypothesized mechanisms and shows the influence connectivity between orthogonally-neighboring legs. These interactions between legs adjust the position of the posterior extreme position (PEP) - the point along the step path where the stance phase transitions into the swing phase based on the weighted summation of connected influences, causing the stance phase to be elongated or shortened.

With Cruse's method, legs generate mechanism values that are weighted and applied as influences on neighboring legs. The legs receiving the combined influences of their neighbors can then have stance/swing (PEP) and swing/stance (AEP) transition points altered (adjusting step length and foot placement) to form a coordinated series of gaits that vary with body speed. This rule set has been used in numerous simulations and robots, such as Robot I (Espenschied et al. 1993), Robot II (Espenschied et al. 1996), Tarry II (Cruse et al. 2003; Frik et al. 
1999), the TUM Walking Machine (Pfeiffer et al. 1994; Weidermann et al. 1994), and BILL-Ant-p (Lewinger 2005; Lewinger, Branicky, and Quinn 2005)

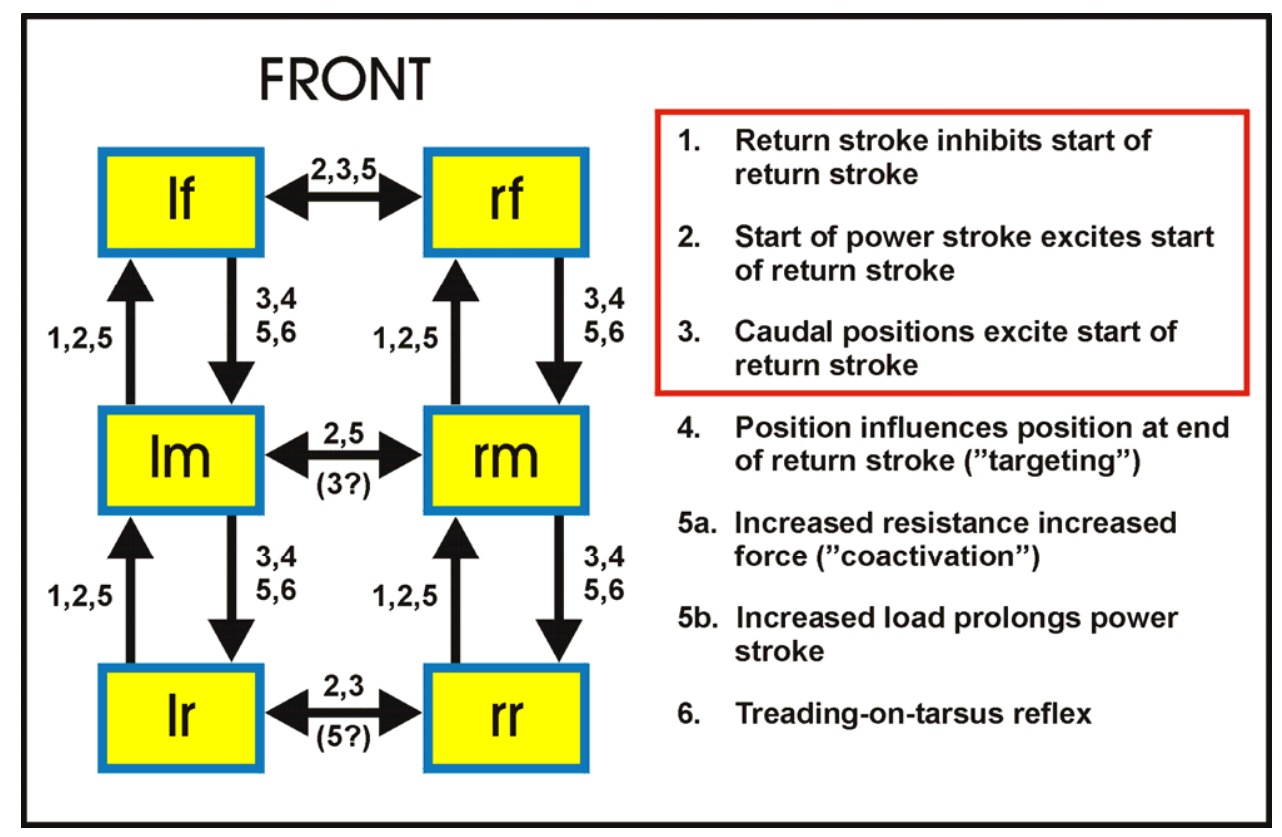

Figure 2.19. Cruse's basic rule set for stick insect walking (Schmitz 1998).

The red box indicates a reduced set of essential mechanisms required for insect gait generation and were used in robots such as Robot I, Robot II, and BILL-Ant-p.

The Cruse method for leg coordination has numerous mechanisms, each with a weighted value of influence on neighboring legs for each connected leg pair. Robot I, Robot II, and BILL-Ant-p each used a subset of these: Mechanisms 1, 2, and 3 (Fig. 2.19, red box). It was determined through previous research (Espenschied et al., 1993) that Mechanisms 4, 5, and 6 were not necessary to create a full range of speed-dependent gaits.

BILL-Ant-p used a parabolic formula and IK to dictate the foot path, and Mechanisms 1, 2, and 3 from Cruse's method for leg coordination (Fig. 2.20) 
(Lewinger 2005; Lewinger, Branicky, and Quinn 2005). The implementation of Cruse's method adjusted the point at which the transition from stance to swing phase occurred for each leg, thereby shortening or lengthening the step. Influences from orthogonally neighboring legs moved the posterior extreme position (PEP) forward or rearward. Once the foot reached the calculated PEP, the transition from stance to swing occurred and a new parabolic foot path for the swing phase was generated.

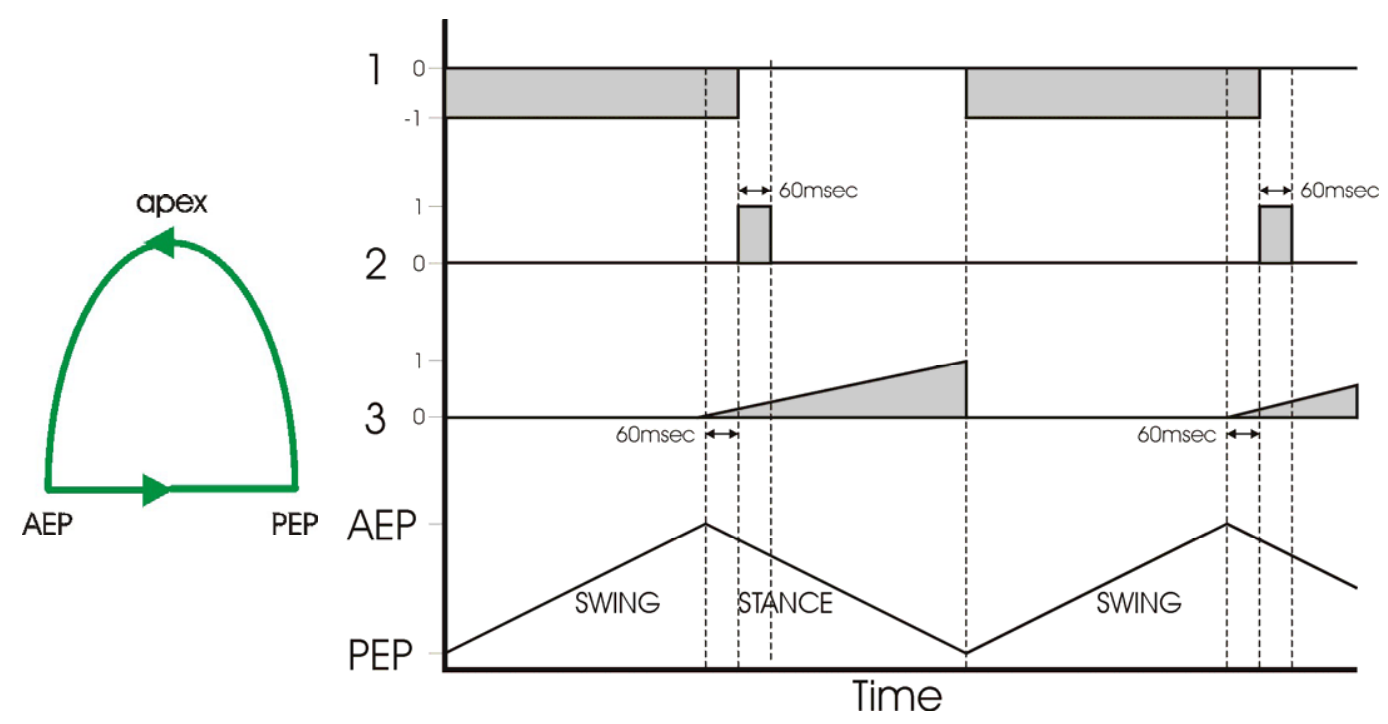

Figure 2.20 Foot path and signal forms for Mechanisms 1, 2, and 3 with respect to leg position as implemented on BILL-Ant-p. (Lewinger 2005; Lewinger, Branicky, and Quinn 2005)

\subsubsection{Walknet}

Walknet is an extension of Cruse's rules for leg coordination in that it ties together an intra-leg joint control artificial neural network (ANN) and an inter-leg coordination ANN. It consists of several connected modules that dictate leg joint velocities (Stance Net and Target Net/Swing Net) and gait behavior (Selector Net) 
based on Cruse's leg coordination mechanisms (Fig. 2.21) (Schmitz et al. 2001; Cruse et al. 2007).

As their names imply, Stance Net and Swing Net control the motion of leg joints when in stance and swing phase, respectively. The outputs of these networks directly control each of the three joint velocities in the legs. They are separate networks and only one has control of a leg's joints at any given time as the leg can only either be in stance or swing. Target Net is used to set swing/stance phase transition locations (the point of achieving ground contact) such that a posterior foot is placed at the same location as that of its anterior neighbor. This behavior is referred to as follow-the-leader, and is useful when navigating sparse terrain.

Based on inputs from neighboring legs and following Cruse's rules for leg coordination, Selector Net decides when the leg should be in stance phase or swing phase, effectively altering the PEP. When the phase changes (e.g., transitioning from stance to swing), the existing net (e.g. Stance Net) is disconnected from the output and the other net (e.g. Swing Net) is engaged. The system's output is a block of summation elements that sends the calculated joint velocity signals from the connected network to the three joints of the leg: $\dot{\alpha}, \dot{\beta}$, and $\dot{\gamma}$ (equivalent to the ThC, CTr, and FTi joints, respectively, of BILL-Ant-a). 


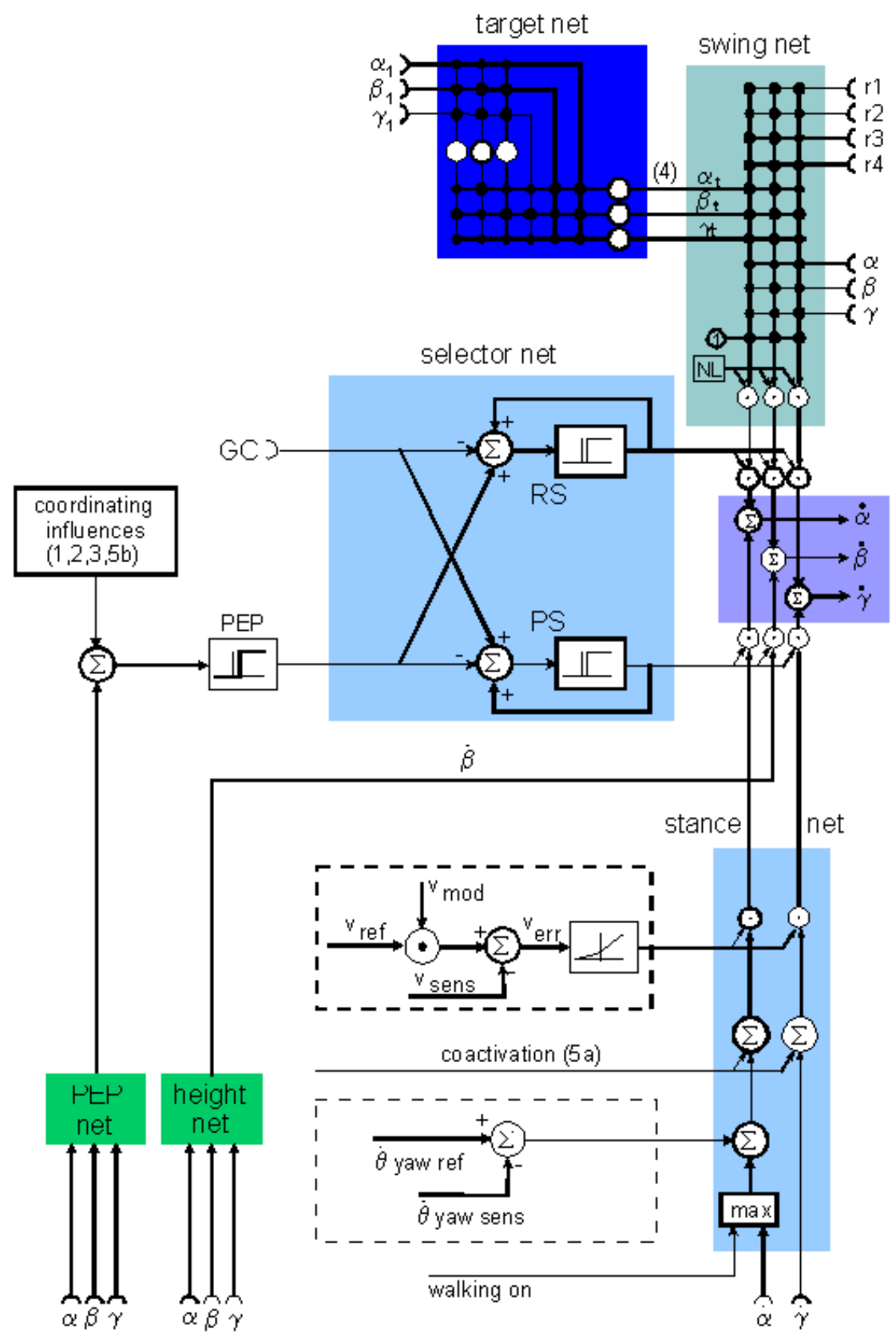

Figure 2.21. Walknet

Walknet is an example of using an artificial neural network (ANN) to coordinate both intra-leg and inter-leg movements. As shown here, Stance Net controls the leg movements when in stance and Swing Net controls the leg movements when in swing; these are the leg joint control portions. For leg coordination, Selector Net uses influence values from Cruse's method to determine when to switch between Stance Net and Swing Net. The outputs of the system are $\dot{\boldsymbol{\alpha}}, \dot{\boldsymbol{\beta}}$, and $\dot{\gamma}$, which are the three joint velocities for a particular leg (Schmitz et al. 2001, Schmitz 1998). 


\section{CHAPTER III}

BiologiCALLY-INSPIRED LEGGED LOCOMOTION - ANT - AUTONOMOUS (BILL-ANT-A)

\section{DESIGN}

\subsection{General}

The BILL-Ant-a robot (Fig. 3.1) uses the BILL-Ant-p robot chassis (Lewinger 2005; Lewinger, Branicky, and Quinn 2005) with a new set of on-board microcontrollers to autonomously sense the environment and actuate the leg joint servo motors in order to navigate uneven terrain while performing phototaxis.

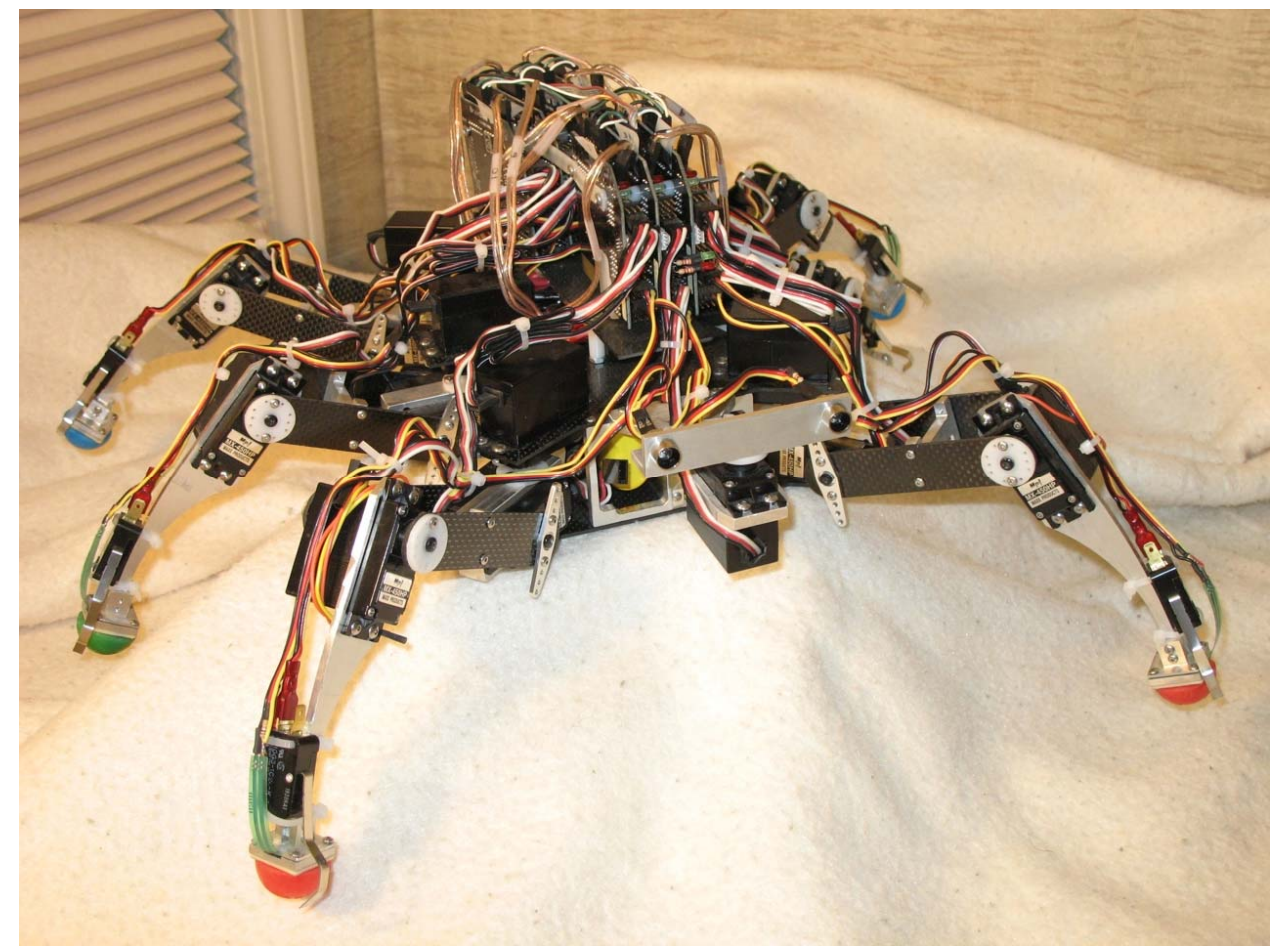

Figure 3.1. BILL-Ant-a hexapod

With on-board leg control microcontrollers (robot top) and neck-mounted stereoscopic photo transistors (robot front). 


\subsection{Physical Design}

As BILL-Ant-a uses the same mechanical chassis as its predecessor, BILL-Ant-p, (without the inclusion of the complete head and neck assembly), most images and descriptions within this chapter will reflect the earlier design.

The BILL-Ant-p base is divided into three major sections: body, legs, and head/neck (Fig. 3.2). Each section is constructed from a 6061 aluminum frame (thickness varying with section) and $0.0625 \mathrm{in}(1.59 \mathrm{~mm})$ thick carbon fiber sheets (McMaster-Carr Supply Co., Cleveland, OH, USA). These materials were chosen for their balance of strength and light weight.
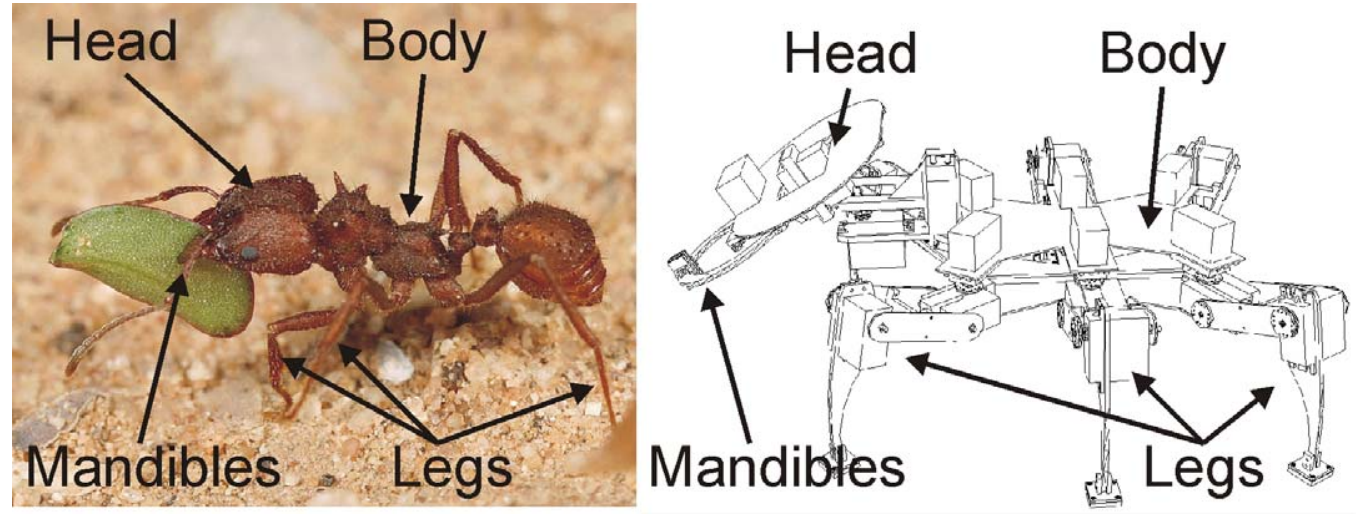

Figure 3.2. Acromyrmex versicolor (left, Leafcutter ant found in Arizona, USA, CDale Ward) and BILL-Ant-p (right) body parts.

Designs were initially created using Autodesk's AutoCAD r14 (Autodesk, Inc., San Rafael, CA, USA). All virtual prototyping for form, placement, range-ofmotion, and interconnectivity was also done with AutoCAD. Final design and machining was done with PTC's Pro/Engineer 2001 (Parametric Technology Corp., Needham, MA, USA). Using the CNC exportation feature, these parts 
were then converted into manufacturing files for fabrication by a Hurco VM1 Machining Center (Hurco Companies, Inc., Indianapolis, IN, USA).

\subsubsection{Body}

The body is the main section of the robot and is used as the anchor point for the legs and neck, and it houses the four on-board batteries (internally) and leg control electronics (mounted to the top) (Fig. 3.3). It is 6.62in (16.8cm) wide, 8.60in $(21.8 \mathrm{~cm})$ long, and 1.93 in $(4.90 \mathrm{~cm})$ tall at the extreme points and is constructed from a $0.1875 \mathrm{in}(4.76 \mathrm{~mm})$ thick 6061 aluminum skeletal frame and has two $0.0625 \mathrm{in}(1.59 \mathrm{~mm})$ thick carbon fiber plates on the top and bottom.

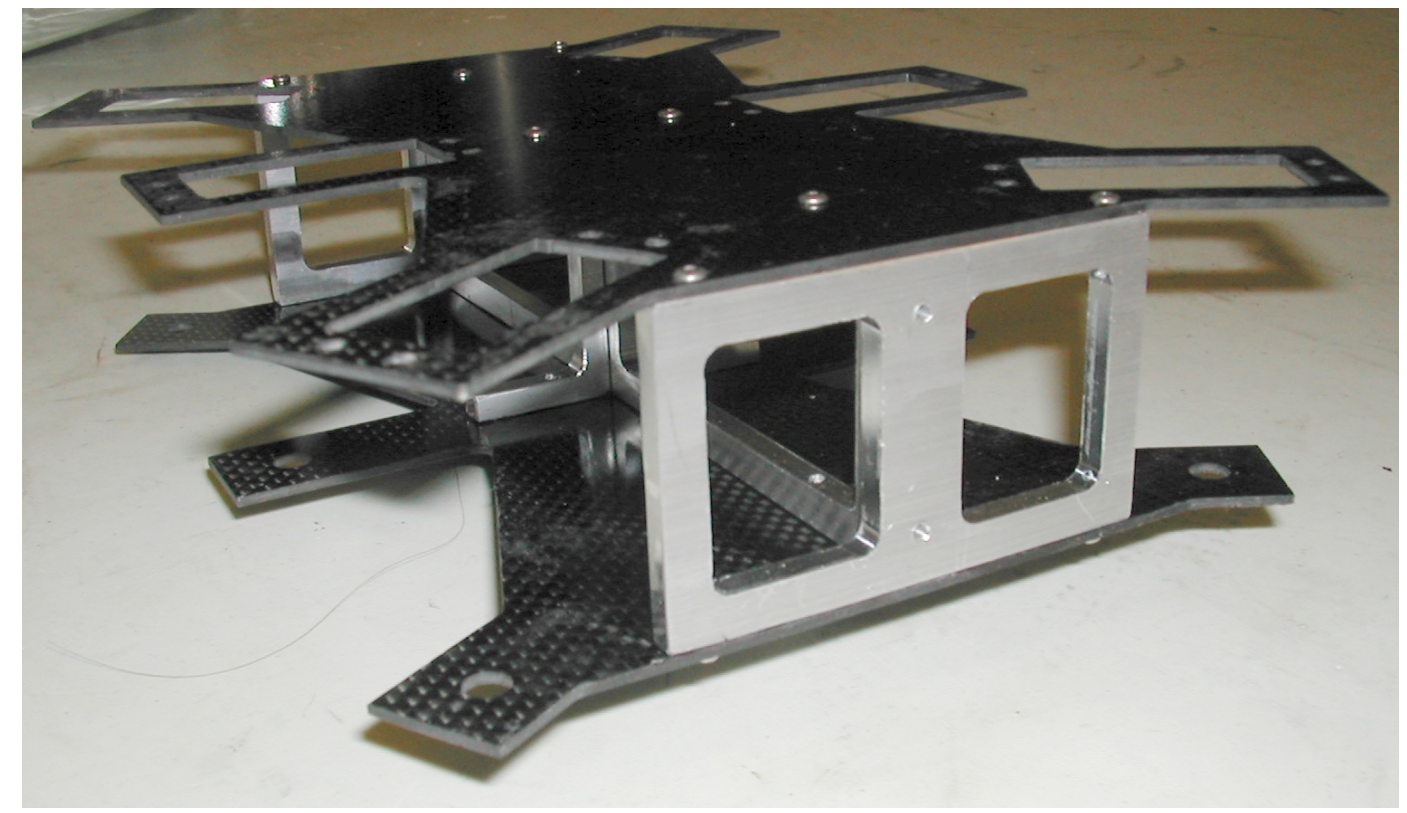

Figure 3.3. Robot body section without legs, electronics, or batteries.

The internal skeleton consists of the spine, front face plate, rear face plate, and two supporting square C-shaped ribs. Each piece is 0.1875 in $(4.76 \mathrm{~mm})$ thick, 1.80in $(4.57 \mathrm{~cm})$ tall 6061 aluminum and has been hollowed to reduce weight. 
The front and rear face plate holes (Fig. 3.3) are used to insert the four Li-ion batteries. The skeleton parts are connected with $\# 2-56$ button socket hex head cap screws that are made of 18-8 stainless steel.

Top and bottom plates are $0.0625 \mathrm{in}(1.59 \mathrm{~mm})$ thick carbon fiber sheets that are secured to the skeleton, giving the body additional rigidity and proving mounting surfaces for other components. The square (Fig. 3.3 top plate) and round (Fig. 3.3 bottom plate) holes are used to mount the thorax-coxa servo motors in an inverted position and support the joint axles, respectively.

Layout of the body and orientation of the ThC joints was based as closely as possible to the body segments of various ants (Fig. 3.4). While the ant has a much more body-compact configuration, the mechanical design was limited by the constraints of function (having space to house the batteries and servo controller) and the connecting elements (providing sufficient space to attach the legs and head/neck assembly). While not as proportionately compact as the insect, the design is as compact as it can be, while still accommodating all of the necessary components. Leg placement and orientation was designed to accommodate 90 degrees of rotation for each $\mathrm{ThC}$ joint (maximum range of motion for the joint motors) while providing minimal interference with other legs throughout the ranges of motion. Front and rear thorax-coxa servos are splayed 60 degrees from the medial plane. The middle ThC joint motors are perpendicular to the medial plane. This pattern is similar to the ant for the middle and rear legs; however, it is not biologically accurate for the front legs. While the 
front thorax-coxa servo orientations were chosen to produce axially-symmetric body plates, the front legs are attached to their respective servos to roughly conform to the ant's anatomy with a starting position of 15 degrees from the medial plane. All legs have \pm 45 degrees of motion; however the front legs have $+0 /-90$ degrees of forward/rearward motion from starting positions of 15 degrees off the medial plane.

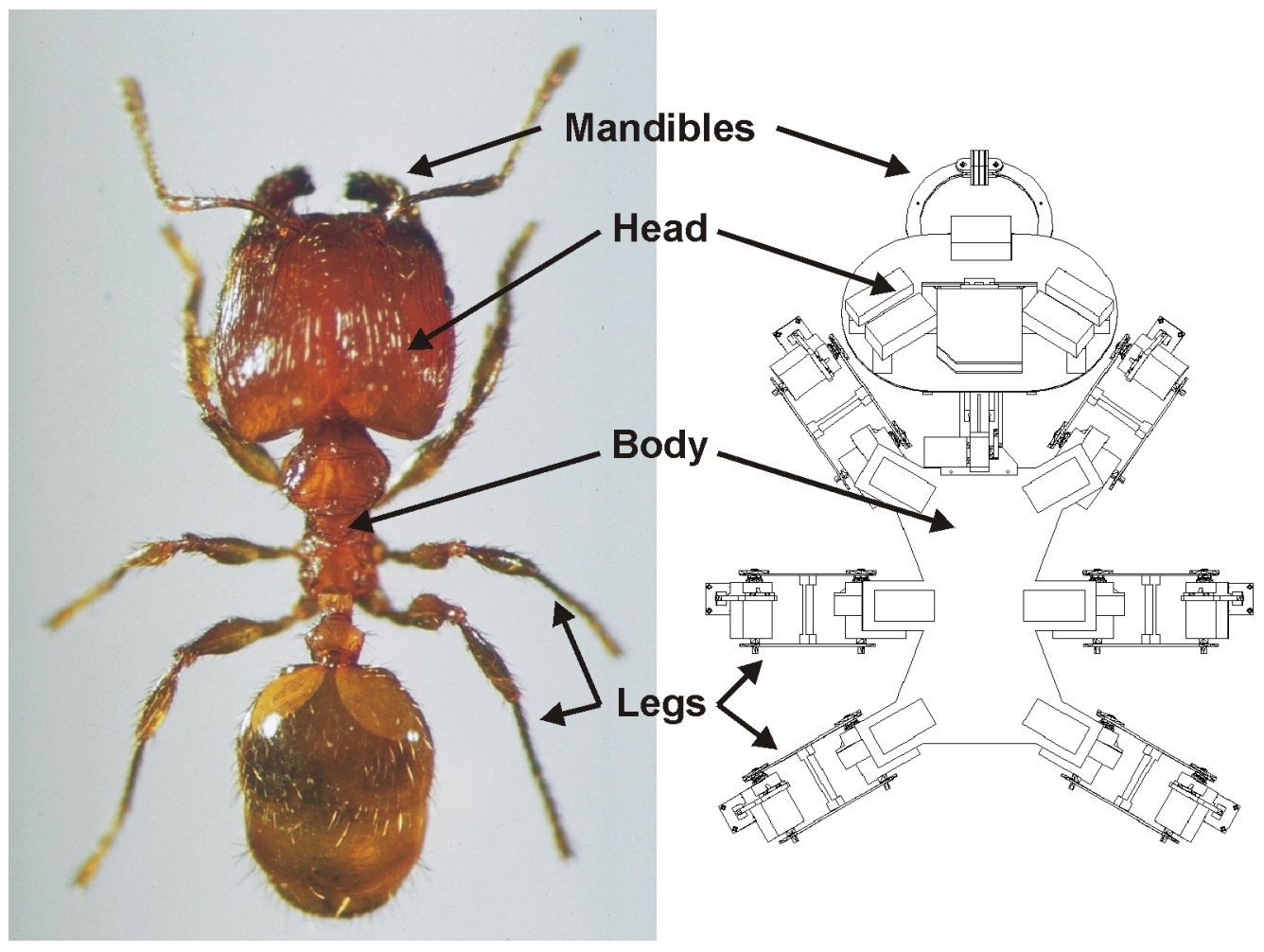

Figure 3.4. Top-view body layout comparison of Pheidole fervida (left, found in Japan, (OJapanese Ant Database Group) and the BILL-Ant-p robot (right).

Body height $(1.93 \mathrm{in} / 4.90 \mathrm{~cm})$ was designed to be as small as possible while still accommodating the Li-ion batteries internally. Due to the limited amount of space on the sides when the legs are present, the batteries are inserted and 
removed through the front and rear face plates. Internal holders (not shown in Fig. 3.3) are used to support the batteries and the voltage conditioning units.

\subsubsection{Legs}

There are six 3-DoF legs on the BILL-Ant-a robot. Three degrees-of-freedom were chosen as that is the minimum number which allows strafing; a desired trait for the robot to enable more agile movements. Additional DoF would have been redundant for basic walking and would have required a greater amount of complexity, power, and processing to control.

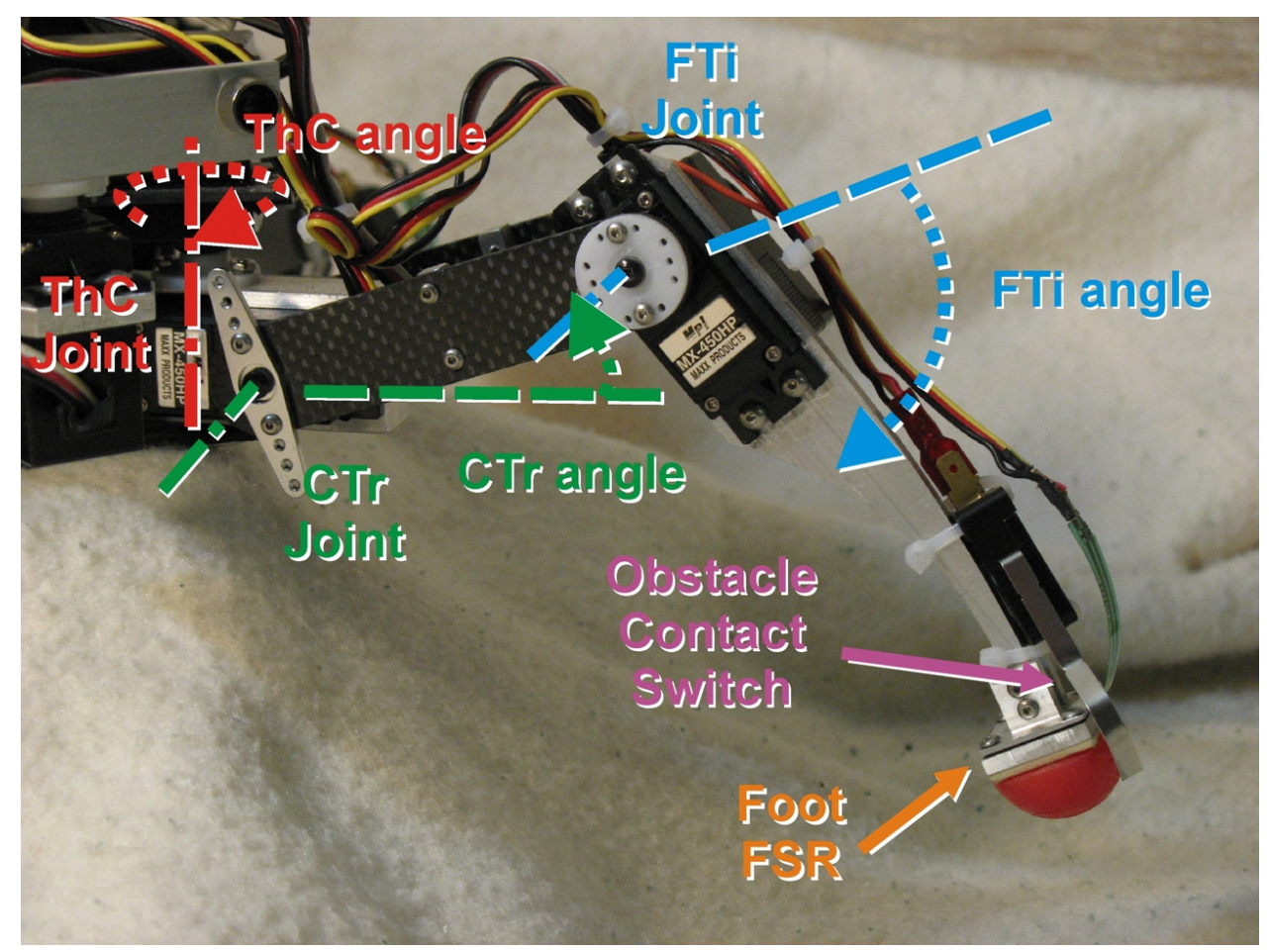

Figure 3.5. Left-front leg of BILL-Ant-a attached to the body with labels.

Each leg consists of three joints and four segments (Fig. 3.5). The first joint is the thorax-coxa (ThC) joint, which swings the leg forward and rearward in the body's dorsal plane. Next is the coxa-trochanter (CTr) joint, which raises and lowers the 
leg in the leg-based medial plane. Finally, the femur-tibia (FTi) joint raises and lowers the tibia and attached foot in the leg-based medial plane.

Hobby R/C servos were chosen as joint motors since the motor, transmission, and position controller are conveniently contained within the servo package. This simplifies the design, construction, and control of the joints while also reducing the cost (when compared to implementing a dc motor, speed controller, and joint angle feedback mechanism).

Several leg prototypes were developed to test the performance of various servo motors available at the time BILL-Ant-p was designed. In the end the MPI MX450HP hobby servo (Maxx Products, Inc., Lake Zurich, IL, USA) was selected. These servos were chosen for reliability, high torque, and affordability. The MPI servos are standard sized (1.60in x 0.80in x $1.49 \mathrm{in}, 4.06 \mathrm{~cm} \times 2.03 \mathrm{~cm} \times 3.78 \mathrm{~cm})$, have a moderate mass of $2.25 \mathrm{oz}(64 \mathrm{~g})$, have $116 \mathrm{oz}$-in $(0.82 \mathrm{Nm})$ of torque, can rotate through a $60^{\circ}$ arc in $0.18 \mathrm{sec}$, and the small internal dc motor consumes $1125 \mathrm{~mW}$ of power at stall torque. While there are more powerful, more intelligent, similarly priced servo motors currently available, the use of many of these models would have required a re-design of the thorax and legs. As such the original mechanical design of BILL-Ant-p was left unchanged for the new robot.

While there are technically four leg segments: coxa, femur, tibia, and foot; the tibia and foot segments are fused to form a contiguous piece, resulting in three moveable links within the leg. 
The coxa is a bracket that houses the CTr servo, and provides anchor points for the ThC servo on one side and the ThC joint axle on the other (Fig. 3.6). The recesses on the interior of the coxae allow the $\mathrm{CTr}$ servo to clear the $\mathrm{ThC}$ joint axle and ThC servo mounting screws.

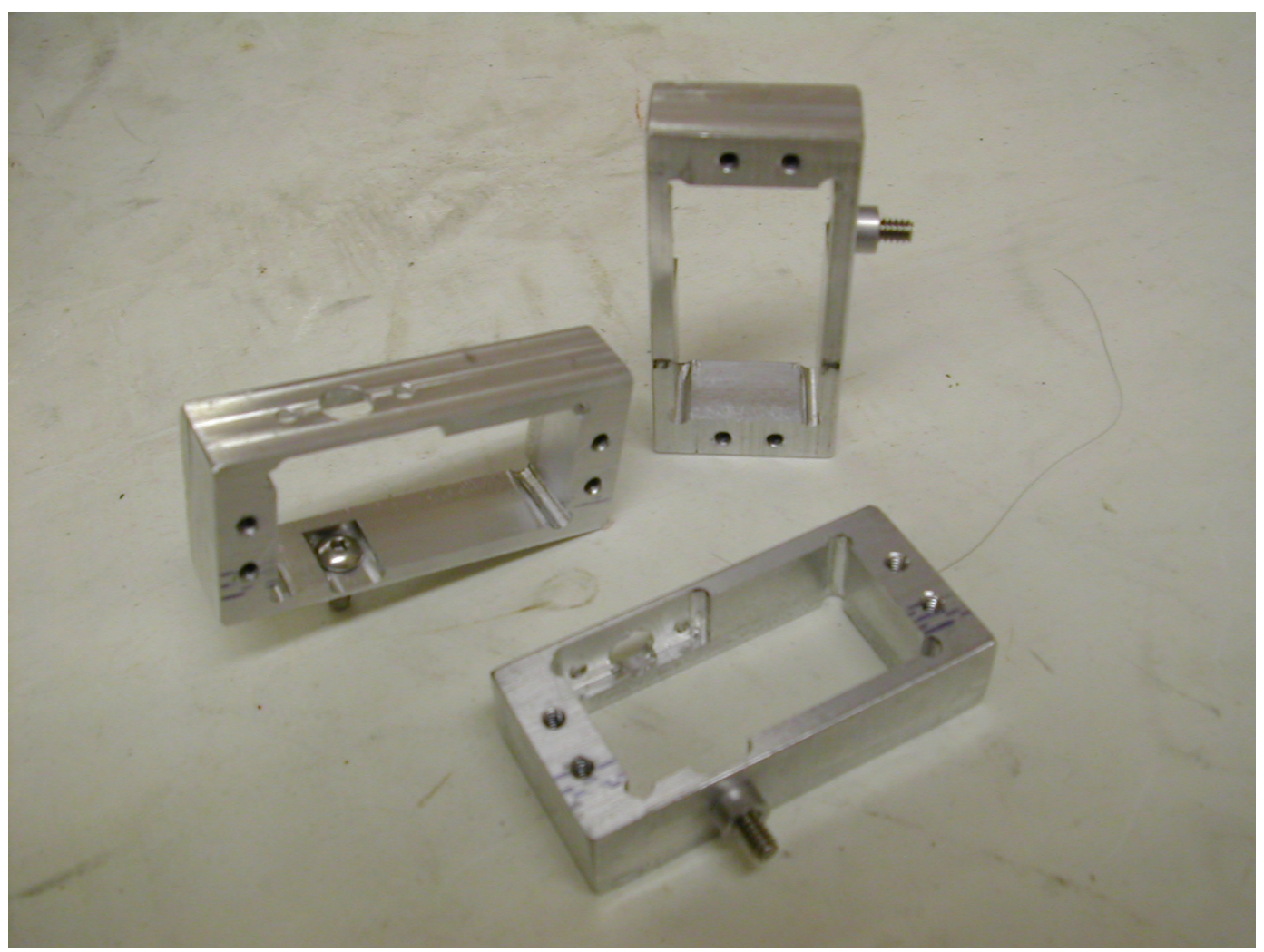

Figure 3.6. Machined coxae with thorax-coxa joint axles installed.

The femur is a double yoke design that connects the CTr joint and the FTi joint (Fig. 3.7). Two $0.0625 \mathrm{in}(1.59 \mathrm{~mm})$ thick carbon fiber plates connect the joint servo horns and joint axles, respectively, and are separated and supported by a 1.66in $(4.22 \mathrm{~cm})$ tall, $0.1875 \mathrm{in}(4.65 \mathrm{~mm})$ thick aluminum strut. Material was removed from the aluminum struts to reduce weight and allow free movement of the $\mathrm{CTr}$ and FTi joint servos. The femur segments are 3.00in $(7.62 \mathrm{~cm})$ axle-toaxle. 
The third leg segment is the tibia, which houses the FTi joint servo and connects directly to the foot. The tibia is constructed from a single piece of $0.125 \mathrm{in}$ $(3.18 \mathrm{~mm})$ thick 6061 aluminum. It is $4.25 \mathrm{in}(10.80 \mathrm{~cm})$ long from the FTi joint axis to the tip, not including the foot.

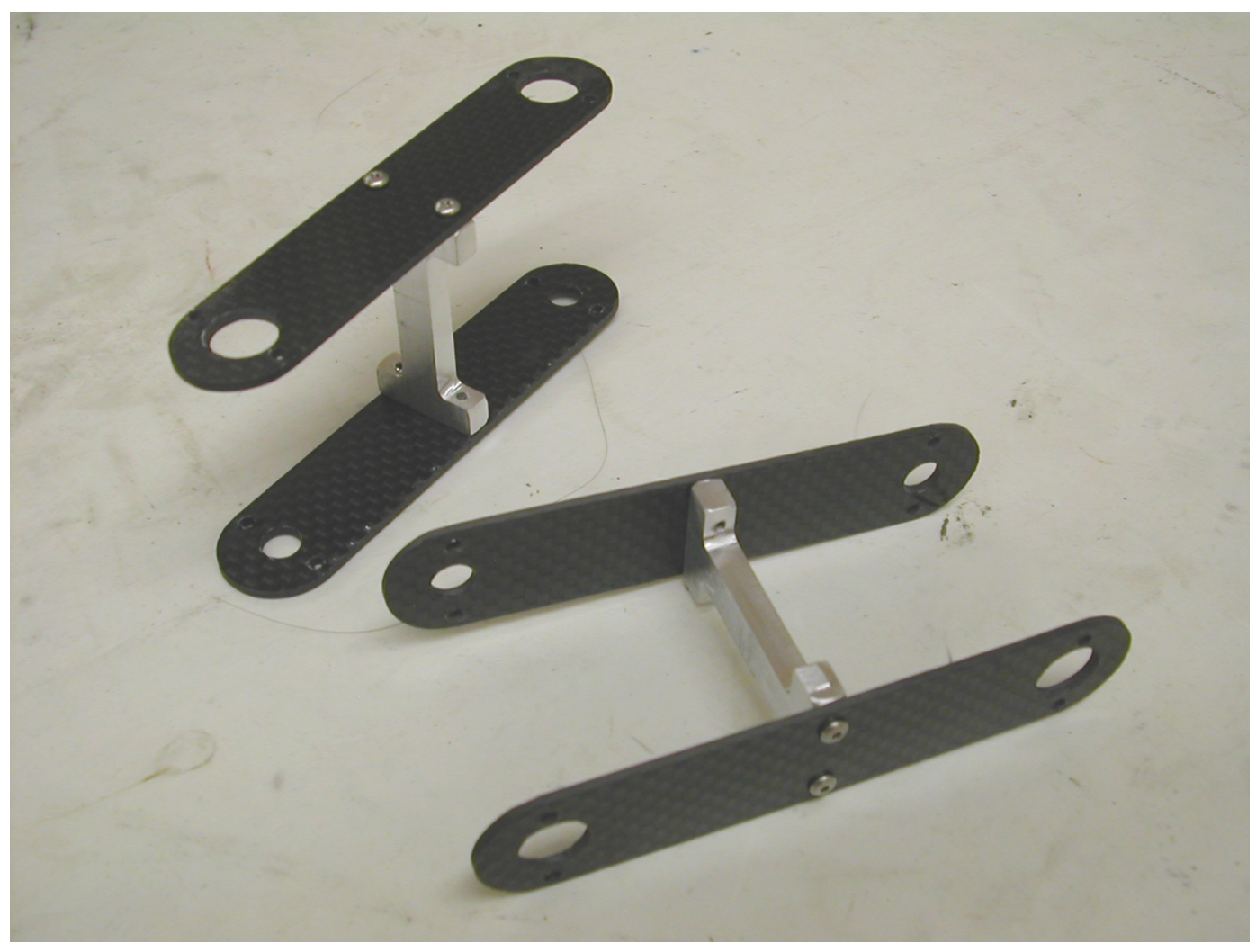

Figure 3.7. Double yoke femurs.

Attached directly to the ends of the tibiae are the feet. The feet provide traction and measure the load along each leg. Each foot is comprised of an Interlink Electronics, Inc. (Camarillo, CA, USA) FSR 402 force-resistive sensor sandwiched between two flat plates, which are $0.8125 \mathrm{in}(2.06 \mathrm{~cm})$ square (Fig. 3.8, left). The upper plate is attached to the end of the tibia, and the lower plate secures the force transducer and supports a 0.5 in $(12.7 \mathrm{~mm})$ long \#4-40 screw protruding from the bottom of the lower plate. An analog-to-digital (ADC) input 
on the associated leg's BrainStem microcontroller is used to measure force at the foot. Resistance-Force characteristics for the FSR 402 are shown in Fig. 3.8 (right). The BrainStem ADC port encodes the force as a 10-bit integer where high forces result in low signals, since the resistance (and the associated voltage drop across the FSR) diminishes as force is applied to the sensor.
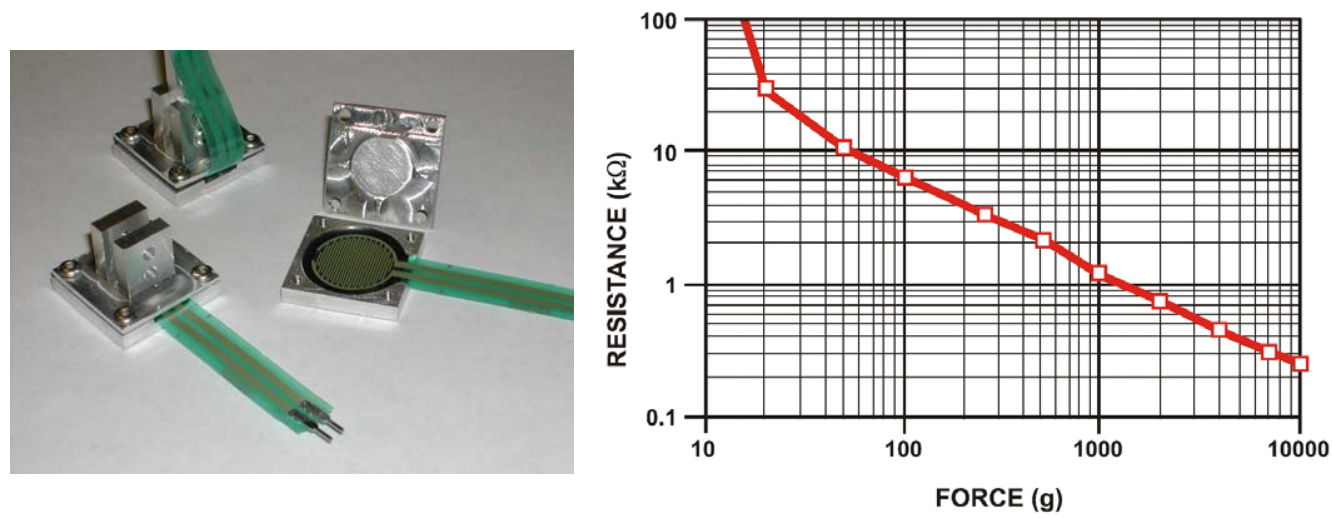

Figure 3.8. BILL-Ant-a foot construction with force-sensitive resistors (left) and the Resistance-Force graph for the FSR elements (right).

The foot-mounted force sensors are used to measure the load observed by each foot. These measurements are compared to two threshold values representing positive ground contact (low value reading), and a definite lack of contact with the substrate (high value reading) to form a hysteresis curve representing state changes in ground contact (Fig. 3.9).

A lever switch is mounted to the distal end of the tibia and the lever is bent to conform to the shape of the foot (Fig. 3.10). This switch is used to detect collisions with obstacles that cannot be cleared by the normal $0.5 \mathrm{in}(1.27 \mathrm{~cm})$ high path of the foot. The level of the switch is bent to fit around the front face of the foot, so it is only triggered when the foot cannot step over an obstacle. The 


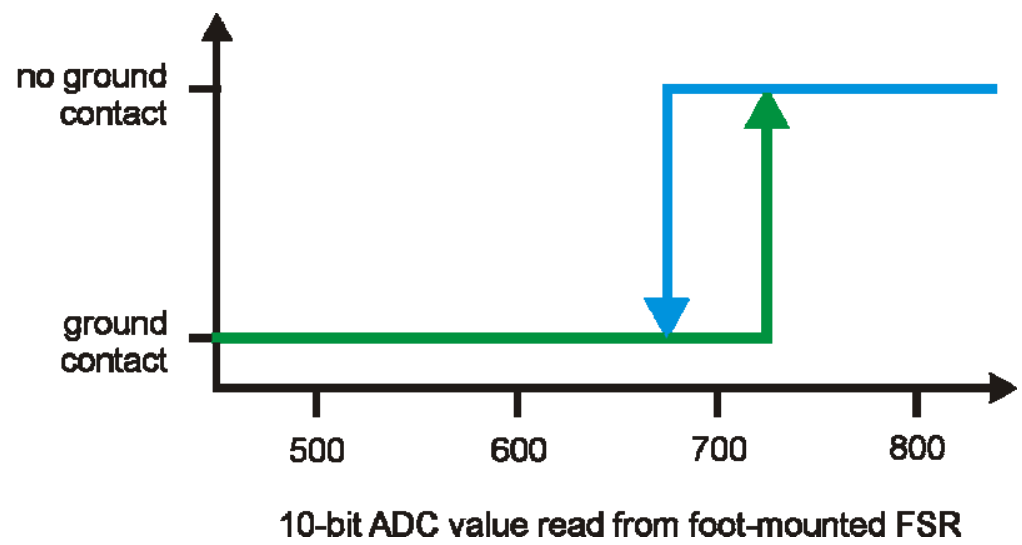

Figure 3.9. The state change diagram shows values from the ADC that allows the leg to determine the condition of contact with the substrate.

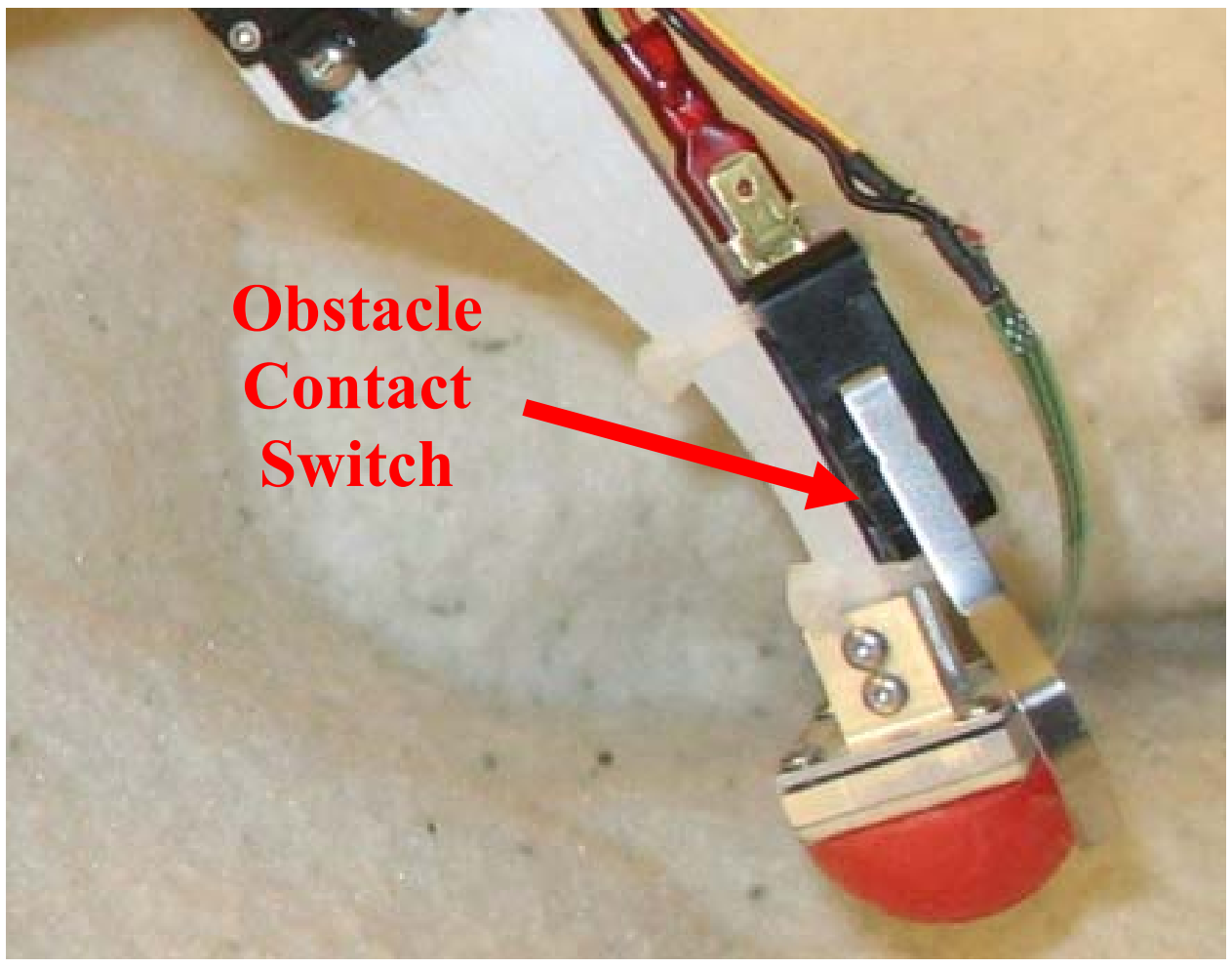

Figure 3.10. Foot-mounted obstacle detection switch

The switch is wired to a simple pull-up resistor network that is read by a digital input on the BrainStem 
switch is wired as part of simple pull-up resistor network, which is read by a digital input port on the BrainStem microcontroller. Contact status is polled by the sensor-reading program on the BrainStem.

\subsubsection{Neck and Light Sensors}
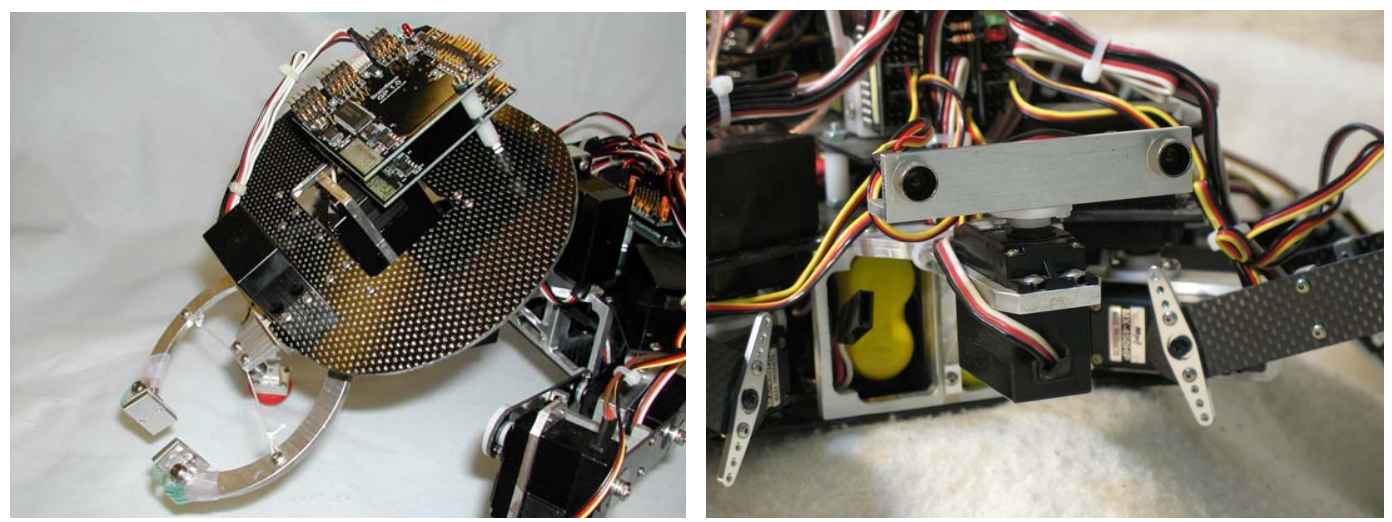

Figure 3.11. BILL-Ant-p head and BILL-Ant-a neck

\section{Original head and neck assembly for BILL-Ant-p with BrainStem microcontrollers and attached mandibles (left), and the new IR sensor module mounted to the neck motor for BILL-Ant-a (right).}

At the front of BILL-Ant-p is the head and neck assembly, which is used to position the attached mandibles that manipulate objects (Fig. 3.11, left). BILLAnt-a is not intended to be used for object manipulation at this time, and so the head and neck assembly have been replaced with an articulated, stereoscopic infrared (IR) sensor module (Fig. 3.11, right).

The sensor module is actuated by the yaw servo motor from the BILL-Ant-p neck assembly. Two IR photo transistors are mounted in an aluminum L-bracket, which is then attached to the servo horn of the neck motor. The two sensors are mounted parallel to one another and are separated by $3.0 \mathrm{in}(7.62 \mathrm{~cm})$. 
Inputs from the two IR sensors are used to sense light-based goals and the "head" BrainStem uses their values to determine the heading of any goals. The neck servo motor is used to actuate the two sensors to point at the goal and can rotate \pm 45 degrees from the central sagittal plane (135 degree total field of view).

\subsection{Electrical System}

The electrical system has two major sections: control and power. The control portion of the system consists of seven networked on-board BrainStem microcontrollers. Power is supplied by four on-board Li-ion batteries, and is provided via two power busses, one for motor power and one for logic power.

\subsubsection{Leg Control System}

The leg control system is comprised of a distributed network of microcontrollers (Fig. 3.12). This network uses individual microcontrollers to control each leg, and an $\mathrm{I}^{2} \mathrm{C}$ communications network for data sharing among the legs. One of the microcontrollers is also designated as a router and is connected to a host PC via an RS-232 serial connection. This allows the host PC to collect data from the leg controllers, or to send specific data or commands to individual legs, joints, or sensors. 

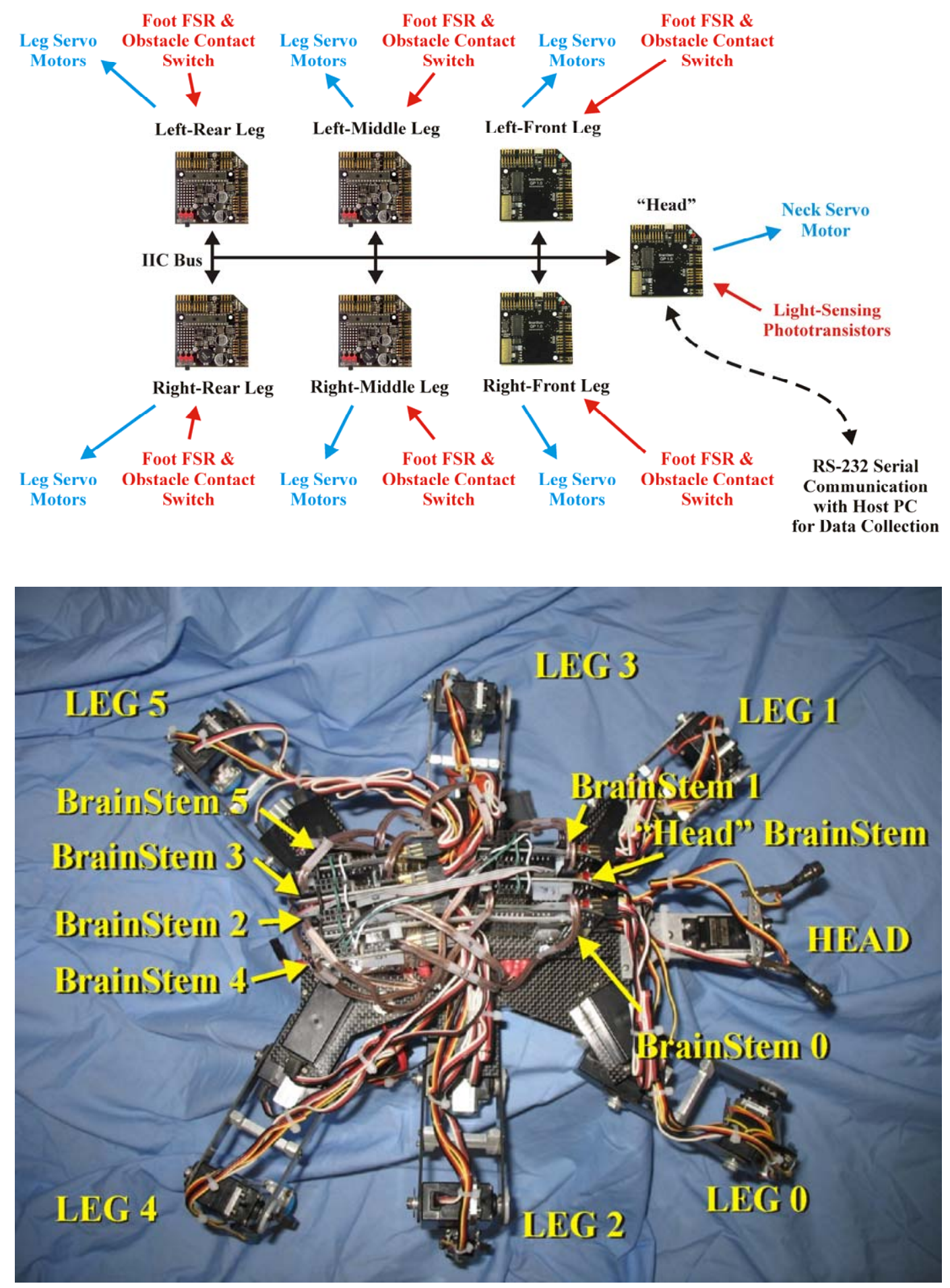

Figure 3.12. Microcontroller Network Schematic and Physical Layouts

(top) Schematic layout of the microcontroller network and $I^{2} \mathrm{C}$ communication bus. (bottom) Physical layout of the seven microcontrollers on the back of BILL-Ant-a as viewed from the top. 
The BILL-Ant-a leg controllers consist of three Acroname BrainStem GP 1.0 and four GP 2.0 microcontrollers (Acroname, Inc., Boulder, CO, USA) (Fig. 3.13). These PIC-based controllers have four R/C servo outputs, five 10-bit ADC inputs, five digital I/O ports, an RS-232 TTL-level serial interface, $\mathrm{I}^{2} \mathrm{C}$ interface bus, and either a digital IR range finder input (for GP 1.0 versions) or an infrared transmitter and receiver pair (for GP 2.0 versions).
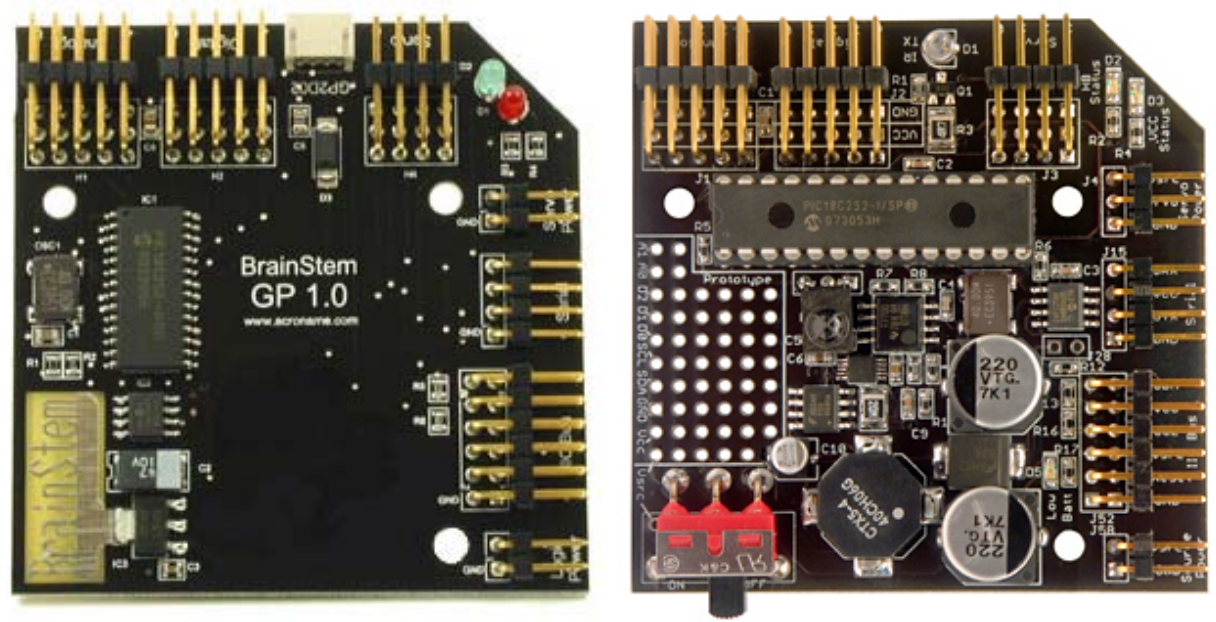

\section{Figure 3.13. Acroname BrainStem GP 1.0 (left) and GP 2.0 (right) microcontrollers (CAcroname, Inc.)}

The microcontrollers have a small power consumption of only 50ma each, which makes them convenient for battery-powered operation. These controllers were selected due to my familiarity with them, the number of $\mathrm{ADC}$ and $\mathrm{R} / \mathrm{C}$ servo channels, their small footprint, and low cost.

The GP 1.0 has a special interface port for a Sharp IR range finding sensor, while the GP 2.0 has an IR transmitter and receiver for communication with other IR devices (such as TV remote controls), instead. Both BrainStem versions have the same model of PIC microcontroller (PIC 18C252 series) and BILL-Ant-a uses a 
combination of the two versions due to a shift in availability from the manufacturer. The mixture of version 1.0 and 2.0 modules does not affect the role or performance of the units; all of the seven microcontrollers are interchangeable with either version.

The BrainStem units can be programmed using a subset of the ANSI-C programming language, a collection of provided functions (such as I/O routines and device interfaces for popular sensors and actuators), and a manufacturersupplied compiler. Each unit has four $1 \mathrm{~K}$ byte (actually 1019 bytes) program slots than can execute code "simultaneously" by sequentially sharing controller clock cycles. The GP 2.0 model has a larger $19 \mathrm{~K}$ size for the first program slot; however this is not exploited in order to maintain continuity among the controllers. Maximum execution speed for a single process is 9,000 instructions per second. For comparison, a typical modern Intel Core 2 Duo microprocessor operates at around 10,000 MIPS - 10 billion instructions per second - six orders of magnitude faster! The slow performance of the microcontroller is useful in demonstrating the computational simplicity of the walking control system (described in Chapter IV and Chapter V).

One of the BrainStem units is designated as the "head" (Fig. 3.11) and is responsible for controlling the 1-DoF neck and for sensing light-based goals (Fig. 3.10, right). A single $\mathrm{R} / \mathrm{C}$ servo output port actuates the neck servo motor, and two ADC input ports are used to read the input values from the pair of IR transistor sensors. 
Four programs are running on this unit. The first is the main program which is responsible for reading the IR transistor values and actuating the neck servo. A small routine is performed that oscillates the neck servo when the light levels are below a set threshold. This seeking behavior continues until at least one of the two light levels rises above the threshold, at which point the program actuates the neck servo in order to balance the two light values for both of the sensor inputs. The position of the neck servo that achieves balanced inputs represents a desired heading for the robot and is used by the remaining three programs. These programs use the heading information to modulate walking control parameters for the legs on the each of the six leg control BrainStem units (described in further detail in Section 4.4).

The remaining six BrainStem units each control one of the six legs (Fig. 3.14). Three of the four R/C servo ports are used to actuate the three leg joint hobby servo motors, a single ADC port reads the signal from the foot-mounted force sensitive resistor (FSR), and a digital input port is used to read the obstacle contact switch value.

While each leg has its own dedicated controller, which is sufficient for implementing the leg control method for a single leg, the six BrainStem units are coupled together via an $\mathrm{I}^{2} \mathrm{C}$ network. This network (Fig. 3.12) allows the leg controllers to share the limited amount of information necessary to orchestrate stable walking gaits, and to receive walking modulation information from the "head" BrainStem. 


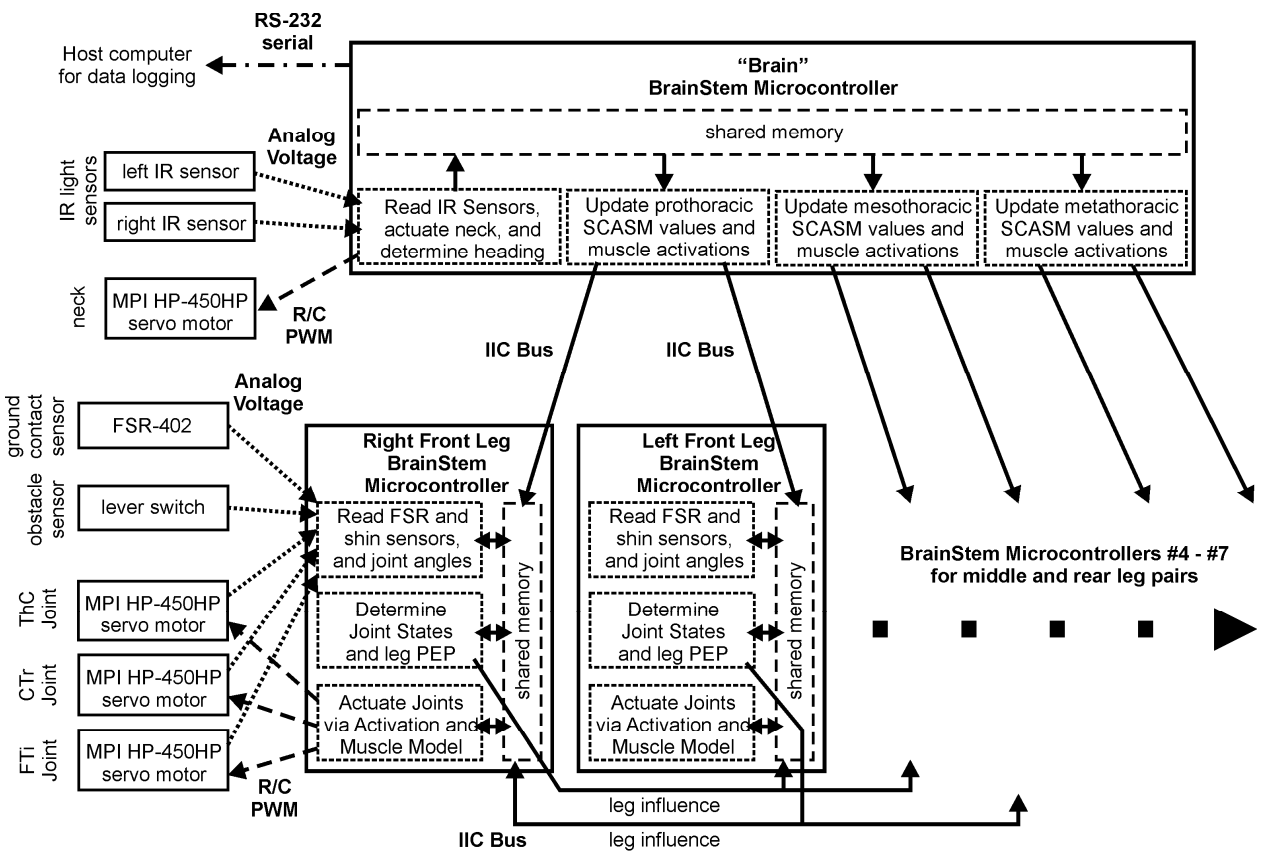

Figure 3.14. BILL-Ant-a electronic and control system connectivity diagram.

The "head" microcontroller also acts as a router for the network when communicating with a host computer during data logging operations. In this configuration, the computer and microcontroller network are connected via an RS-232 cable with an in-line level-shifter module that converts the PC-side serial signals into BrainStem-compatible TTL levels.

The BrainStem network is stacked into a column of three and a column of four microcontrollers, each board separated from the next by a $0.5 \mathrm{in}(12.7 \mathrm{~mm})$ nylon spacer and secured by \#4-40 stainless steel screws. Nylon angle brackets are installed between one pair of boards in each column, and are secured to a small rectangle of carbon fiber that allows the microcontrollers to sit vertically on the back of the robot. The carbon fiber plate is secured above the power distribution terminal block (described below in Section 3.2.2) and connections are routed to the microcontroller logic and servo power ports. 22-gage wire cables were 
created to couple each of the $\mathrm{I}^{2} \mathrm{C}$ network ports, and the joint servo motors and sensor cables are connected to their respective input and output ports on their respective BrainStems (Fig. 3.15).

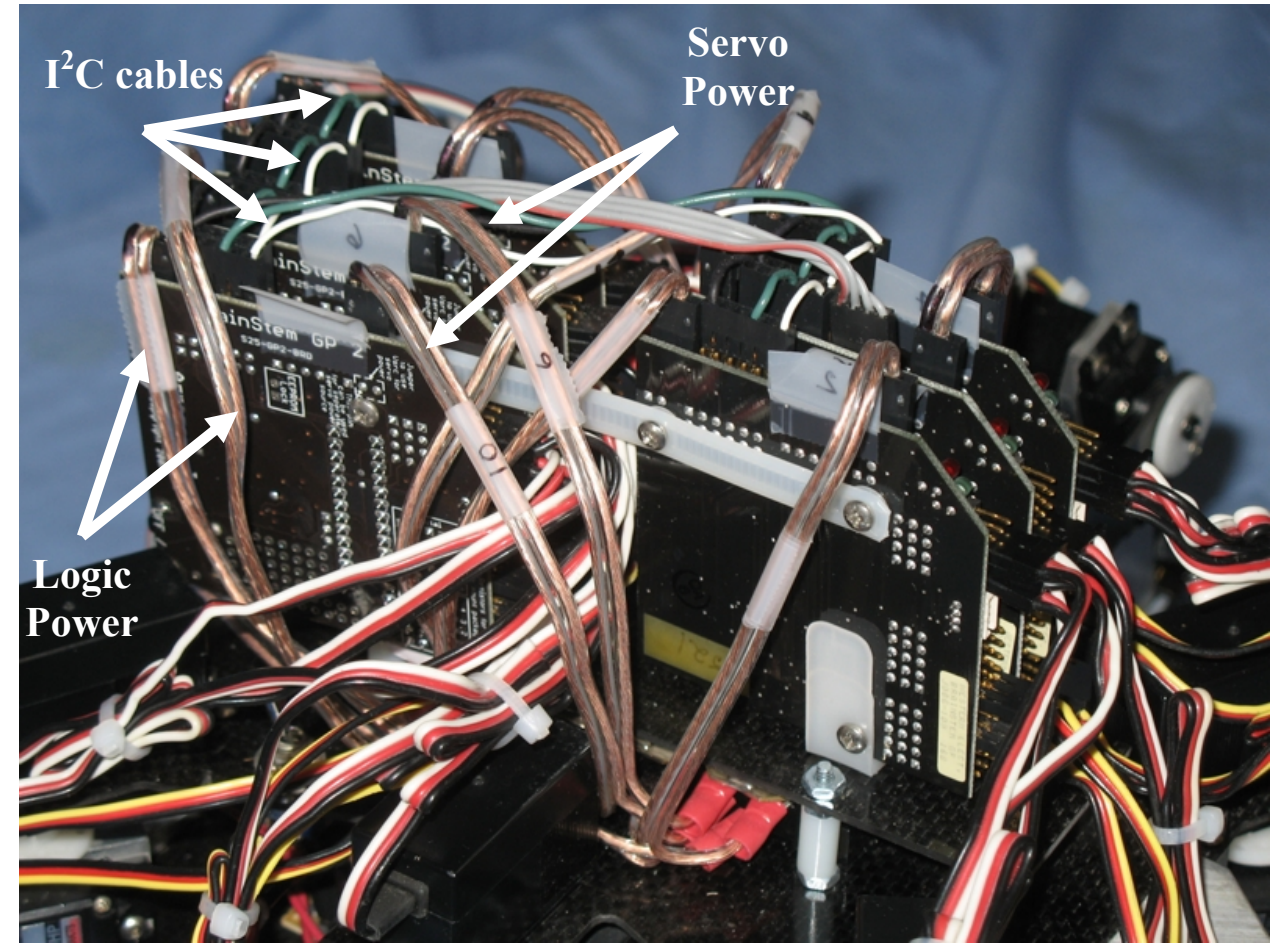

Figure 3.15. BrainStem microcontroller network for BILL-Ant-a.

Shown from the right-front leg perspective, the three microcontrollers on the right are for the front legs and the "head"; the four BrainStems on the left are for the remaining legs. Stranded wire pairs with clear insulation are from the power distribution busses for logic and motor power. Black-redwhite cables are from joint servo motors and black-red-yellow cables provide connections to the foot-mounted FSR and obstacle contact switch sensors.

\subsubsection{Power System}

Four two-cell 2400mAH 7.2vdc Li-ion batteries from Maxx Products, Inc. (MPI, Lake Zurich, IL, USA) are used to power the robot. The batteries are stored inside the body to provide power autonomy. 
One of the batteries powers the logic portion of BrainStem microcontrollers, since they require relatively little power. The other three batteries are connected in parallel to supply power for all of the servo motors via the motor power connection on the BrainStems. The Li-ion batteries have a greater instantaneous current delivering capacity and a better power density than NiMH batteries. As such, the Li-ion batteries are smaller than NiMH batteries with similar power ratings. The $2400 \mathrm{mAH}$ capacity allows for operation of up to $36 \mathrm{~min}$, depending on current demands of the motors.

To limit the delivered voltage to $6.0 \mathrm{vdc}$, each of the batteries is connected to an MPI ACC134 6-volt Regulator. These units step the 7.2vdc (and higher when freshly charged) voltages down to $6.0 \mathrm{vdc}$, which is the maximum safe voltage for use with standard hobby servos. Since the voltage regulators can provide $10 \mathrm{~A}$ continuously and $20 \mathrm{~A}$ peak, there is no current throttling of the Li-ion batteries when using these regulators. Although one regulator may be sufficient on level terrain where the operating current range is approximately $4.0 \mathrm{~A}-7.0 \mathrm{~A}$, irregular terrain navigation and climbing tasks can require up to $10 \mathrm{~A}$, or slightly more. So, for the assurance that the power regulator will not hinder the current-delivering capacity of the batteries, each battery has its own regulator.

For safety, the servo power bus has an in-line 15A fuse. Although there is noticeable heat generated in the servos cases and slight warmth in the Li-ion batteries, the $15 \mathrm{~A}$ fuse has neither burned out, nor allowed too much current to damage system parts. 
Two power switches are mounted on the top of the robot: one for servo motor power from the three-battery bus, and one for logic power (power for the microcontrollers). Servo power and logic power are routed from the switches to an 8-pair terminal block. Each mounting point of the terminal block contains spade connectors that route power and ground. Four terminal points are electrically coupled to form a servo motor power bus, and an additional four terminal points are coupled to form a logic power bus, and the remaining eight terminal points form a common ground bus for both servo motor and logic power. 16-gage wire and spade connectors are used to distribute and supply power from the terminal block to the BrainStem logic and servo power ports.

For testing purposes, either the on-board batteries or an off-board bench top power supply can be used. 


\section{CHAPTER IV}

\section{LEG CONTROL}

\section{SENSORY-Coupled ACTION Switching Modules (SCASM)}

\subsection{General}

In the design of competent, agile robots, engineers often look to biological systems as a source of inspiration. Various aspects of natural systems can be used for this inspiration, such as physical appearance, mechanical design, and control methods. To varying degrees, these aspects have been implemented in mobile robots.

One of the critical aspects of legged locomotion is the generation of joint motions that produce coordinated actions within each leg. It is this coordination that gives rise to fluid stepping motions. Previously, this has been accomplished by replicating the observed motions of insect legs, using methods such as inverse kinematics, central pattern generators, or artificial neural networks to generate the joint actions. Unfortunately, these methods can be too computationally expensive for most microcontrollers and do not represent the actual manner in which biological systems create these stepping motions.

Relatively recently, however, Ekeberg, Blümel, and Büschges (Ekeberg et al. 2004) have modeled an identified network in the thoracic ganglia that coordinates 
the joints of a leg in a stick insect, which introduces the possibility of computationally and conceptually simple intra-leg joint control.

\subsection{Biological Basis for Leg Control}

The generation of walking behaviors is demanding because it must be performed by a mechanically complex, multi-limbed and multi-segmented locomotor system navigating an unpredictable environment. In order for a legged system to successfully produce such behaviors it needs precision control over the movement of all its limbs. Animals create movements by activating muscles that have a series of non-linear dynamic properties that can make them difficult to control accurately. The required precision is only obtainable by having sensory feedback in concert with muscle control. The sensory input includes kinematic properties like joint positions and velocities in addition to signals reporting dynamic properties like force, strain, and contact with other objects. Sensory information is indispensable to generate functional locomotor behavior in such a system.

The body and various legs of a walking system must coordinate with one another to prevent the animal from stumbling or falling. Equally important is the coordinated movement of the individual leg segments to carry and propel the body during stance phase and return to a proper starting position in swing phase. Evidence in the identified leg muscle control system of the stick insect suggests that the neuronal control can be subdivided into several central pattern generators (CPGs), one for each joint. A CPG generates rhythmic motor output, even without any peripheral input. Each CPG can generate a basic alternating activity 
pattern in antagonistic muscles of a given leg joint, thus creating an oscillatory extension and flexion cycle. It is possible to activate these CPGs either pharmacologically or by tactile stimulation of the animal (Büschges et al. 1995; Büschges et al. 2004). Experiments with pharmacological activation of the leg control network led to the conclusion that each of the three primary leg joints (ThC, CTr, and FTi) can be associated with its own, individual CPG. This is based on the observation that the joint muscles show alternating activity (oscillations) under pharmacological activation but do not show the cycle-to-cycle coupling between the oscillations of muscles controlling different joints that is necessary to create stepping motions. Signals of sense organs can either change the probability of the CPG to stay in a given state (timing influences) or shape the amplitude of the motor output (magnitude influences) (Büschges 2005).

In order to be able to generate a stepping motion the activity of the three joint control CPGs must be coordinated. This coupling is achieved by means of sensory feedback (Atay et al. 2001; Atay et al. 2004; Bucher et al. 2003; Hess and Büschges 1999). Usually, leg proprioceptors can be attributed to a specific leg segment and therefore can be associated with the joint that moves this segment. If a sense organ influences the CPG of its associated segment, then it is called an intra-joint influence; if it affects the activity in other joints it is referred to an inter-joint influence. The chordotonal organ, for example, is a stretch receptor. It is able to transmit information about a joint's current geometry, like joint angle or angular change (Bässler 1993). Signals from the FTi chordotonal organ do not only influence the CPG of the FTi joint itself (Bässler and Büschges 1993), but 
also the motor-activation of the adjacent (CTr) joint (Bucher et al. 2003; Hess and Büschges 1999). These inter-joint influences are an important mechanism of segment coordination.

The role of individual leg sense organs in the control of motor output for stepping has been studied in great detail (Büschges 2005; Bässler and Büschges 1998). In order to cope with the complexity of this topic, many experiments have been performed using so called "reduced preparations". In these preparations one tries to approach operational isolation of the behavior of interest by inactivation or removal of dispensable biological functionality. In case of the stick insect a popular reduced experimental preparation is the single leg preparation, with only one leg remaining attached to the animal (Bässler et al. 1991; Fischer et al. 2001). Often the remaining leg is further limited in its degrees of freedom by preventing forward and backward movements (this is referred to as the restricted single leg preparation). This experimental setup creates a movement that is sometimes referred to as "sideways walking".

\subsection{Introduction to Sensory-Coupled Action Switching Modules (SCASM)}

The results of investigations into insect neurophysiology form the basis for developing a new model of leg movement control: the Sensory-Coupled Action Switching Modules (SCASM). In this model, control of the 3-DoF leg is split into three independent joint control systems. Each joint controller is similar to a state machine in that its simple task is to determine whether the joint should be in

either extension or flexion. Of course, joint control is not as simple as that, and 
requires the regulation of joint speed and force as well. For the moment, however, only the joint direction will be discussed. To make this decision the joint controller has access to specific sensor data, but it has explicitly no information about the state of other joint controllers (Ekeberg et al. 2004). This reflects the uncoupled nature of the biological archetype.

Since each joint can only be in either extension or flexion, it is possible to simplify the joint controller's task to a set of Boolean decision rules, for example:

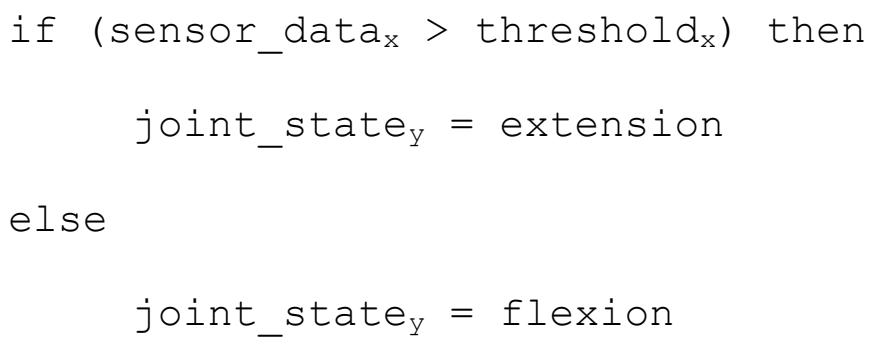

In case of the stick insect the appropriate rules for each joint can be derived almost completely from the literature; only the threshold values have to be fitted to the given leg geometry.

BILL-Ant-a and the test platforms described below use the same basic control architecture as in (Ekeberg et al. 2004) which is represented in Fig. 4.1. The leg segments, from the body outward, are the coxa, femur and tibia. We maintain this nomenclature for both the stick insect scale model and the general 3-DoF leg.

When considering joints located from the thorax to the foot, the first and simplest to control of the joints is the thoraco-coxal (ThC) joint. The ThC joint relies only on ground contact to transition between protraction (swing) and retraction 


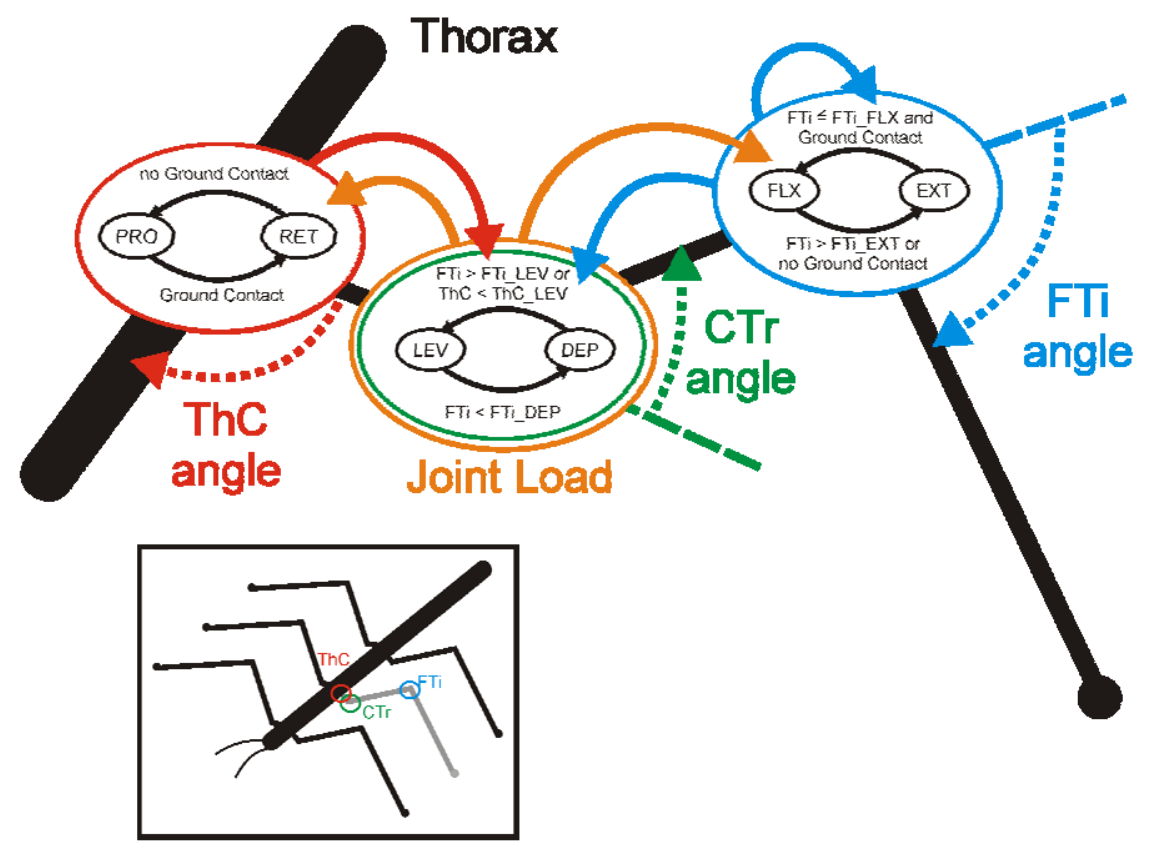

Figure 4.1. Original Sensory-Coupled Action Switching Modules (SCASM) system connectivity diagram. (Lewinger, Rutter, et al. 2006).

Diagram of geometry and control of the simulated stick insect middle leg, showing ThC (Thoraco-Coxal) protraction and retraction, CTr (Coxa-

Trochanter) levation and depression, and FTi (Femur-Tibia) flexion and extension. The conditions used by Ekeberg et al. 2004 for transitions between states are shown adjacent to each black state transition arrow.

(stance). Ground contact is detected in one of two ways. For the Single- Leg Test Platform, described below in Section 4.3.1, ground contact is sensed by reading the electric current (a proxy for joint load) signal from the $\mathrm{CTr}$ joint servo motor; the special motors used in that platform allow bi-directional communications for data and position commands. For the Two-Leg Test Platform and BILL-Ant-a, the foot-mounted force-sensitive resistor (FSR) is used to determine the load on the foot. When the sensed voltage value is below a threshold (increasing the force acting on the FSR reduces its voltage and produces a lower voltage output), ground contact is assumed. As the load reduces and the 
voltage rises above a second threshold, the leg is assumed to no longer be in contact with the ground (Fig. 3.9).

Relying solely on inter-joint angle information, the coxa-trochanter (CTr) joint (i.e. the second joint), receives sensory input from both the ThC and FTi joints to determine its state. This joint levates the leg in order to aid the transition from stance to swing phase. In the typical model, this levation state persists until the FTi joint extends sufficiently to initiate a change to the depression state. For BILL-Ant-a, an additional condition is presented such that either the FTi joint extending beyond a threshold or the ThC joint protracting forward of a setpoint can initiate depression. This alteration was made to provide more reliable stepping motions given the slow processing speed of the BrainStem microcontrollers. The transition from levation to depression occurs roughly in the middle of swing phase (Fig. 4.2, green line). The joint then changes from depression to levation when either the FTi joint flexes within a threshold or the ThC joint retracts past a set angle. This change of states occurs near the middle of stance phase in order to unload the foot in preparation for the next swing phase.

The third joint, the femur-tibia (FTi) joint uses both joint angle and ground contact information to determine its state. When the joint is in extension, a transition to flexion occurs when both ground contact is detected and the FTi joint angle has extended beyond a threshold. This occurs at the beginning of stance phase (Fig. 4.2, dashed red line). There is then a switch to extension that occurs near the middle of the stance phase when the joint flexes past another setpoint. 
The joint also remains in the extension state while ground contact is lost during swing phase. This allows the leg to reach toward new footholds for the next stance phase.

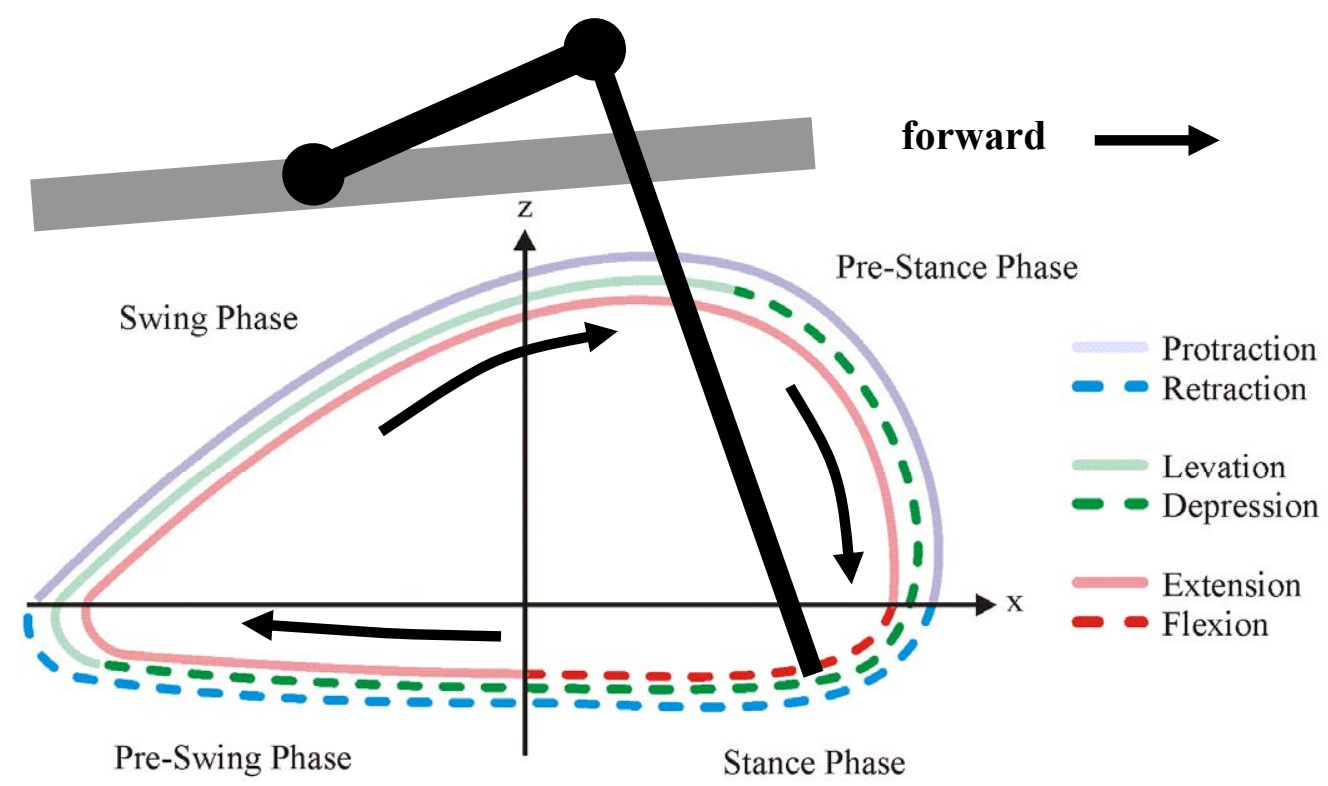

Figure 4.2. Foot path and joint states for a typical step cycle.

\section{The ground level line $(\rightarrow \mathbf{x})$ represents the ground contact position for an unloaded leg.}

While these state transitions hold for the front and middle legs, there is a slight modification seen in the rear legs. Due to the role of the rear legs, whose purpose is to propel either the insect or the robot, the extension and flexion actions are reversed, as noted in Ekeberg et al. So, during the beginning of stance phase, detection of ground contact and an FTi joint angle flexed beyond a threshold trigger a change to the extension state. Similarly, while in stance, extending past another setpoint causes the transition to flexion. The flexion state persists while the leg is in swing and no ground contact is sensed. This reversal of behaviors for the FTi joint is sufficient to alter the actions of the rear leg from a reaching 


\begin{tabular}{|c|c|c|c|c|}
\hline \multirow{2}{*}{ Joint } & \multirow{2}{*}{ State } & \multicolumn{2}{|c|}{ Joint Angles (degrees) } & \multirow{2}{*}{$\begin{array}{l}\text { Ground } \\
\text { Contact? }\end{array}$} \\
\hline & & ThC & FTi & \\
\hline \multicolumn{5}{|c|}{ Original Ekeberg et al. Values } \\
\hline \multirow[t]{2}{*}{ ThC } & $\mathrm{PRO} \rightarrow \mathrm{RET}$ & -- & -- & yes \\
\hline & $\mathrm{RET} \rightarrow \mathrm{PRO}$ & -- & -- & no \\
\hline \multirow[t]{2}{*}{$\mathrm{CTr}$} & $\mathrm{LEV} \rightarrow \mathrm{DEP}$ & -- & $<70$ & -- \\
\hline & $\mathrm{DEP} \rightarrow \mathrm{LEV}$ & $<-25$ & $>120$ & -- \\
\hline \multirow[t]{2}{*}{ FTi } & $\mathrm{EXT} \rightarrow \mathrm{FLX}$ & -- & $\leq 105$ & yes \\
\hline & $\mathrm{FLX} \rightarrow \mathrm{EXT}$ & -- & $>105$ & no \\
\hline \multicolumn{5}{|c|}{ Single-Leg Test Platform } \\
\hline \multirow[t]{2}{*}{ ThC } & $\mathrm{PRO} \rightarrow \mathrm{RET}$ & -- & -- & $>0.5^{*}$ \\
\hline & $\mathrm{RET} \rightarrow \mathrm{PRO}$ & -- & -- & $<-0.5^{*}$ \\
\hline \multirow[t]{2}{*}{$\mathrm{CTr}$} & $\mathrm{LEV} \rightarrow \mathrm{DEP}$ & & $<80$ & -- \\
\hline & $\mathrm{DEP} \rightarrow \mathrm{LEV}$ & $<-25$ & $>115$ & -- \\
\hline \multirow[t]{3}{*}{ FTi } & $\mathrm{EXT} \rightarrow \mathrm{FLX}$ & -- & $\leq 100$ & $>0.2 *$ \\
\hline & $\mathrm{FLX} \rightarrow \mathrm{EXT}$ & -- & $>120$ & $<-1^{*}$ \\
\hline & $\mathrm{EXT} \rightarrow \mathrm{EXT} 2 \dagger$ & -- & $<80$ & $<=0 *$ \\
\hline \multicolumn{5}{|c|}{ Two-Leg Test Platform and BILL-Ant-a } \\
\hline \multirow[t]{2}{*}{ ThC } & $\mathrm{PRO} \rightarrow \mathrm{RET}$ & -- & -- & yes \\
\hline & $\mathrm{RET} \rightarrow \mathrm{PRO}$ & -- & -- & no \\
\hline \multirow[t]{3}{*}{$\mathrm{CTr}$} & $\mathrm{LEV} \rightarrow \mathrm{DEP}$ & $>35$ & $<48$ & -- \\
\hline & $\mathrm{DEP} \rightarrow \mathrm{LEV}$ & $<-35$ & $>107$ & -- \\
\hline & $\mathrm{DEP} \rightarrow \mathrm{LEV} \S$ & $<-90$ & $>180$ & -- \\
\hline \multirow[t]{3}{*}{ FTi } & $\mathrm{EXT} \rightarrow \mathrm{FLX}$ & -- & $<46$ & yes \\
\hline & $\mathrm{EXT} \rightarrow \mathrm{FLX} \S$ & -- & $<0$ & yes \\
\hline & $\mathrm{FLX} \rightarrow \mathrm{EXT}$ & -- & $>90$ & no \\
\hline
\end{tabular}

Table 4.1. SCASM state transition threshold values.

* Numerical values from the analog-to-digital converter that represent the sensed load in the CTr joint, used to detect ground contact in the Single-Leg Test Platform

$\dagger$ Additional state added to improve performance before a "muscle model" was used

§ Special, unreachable values when the other leg is in swing; prevents more than one leg being in swing at a time 
("pulling") role, as seen in the front and middle legs, to a propelling ("pushing") role, typical of insect rear legs. Table 4.1 shows the state transition threshold values and ground contact conditions required to change between states for each of the three leg joints.

Values are listed for the original Ekeberg et al. system, the Single-Leg Test Platform, the Two-Leg Test Platform, and BILL-Ant-a. The values indicated in the table represent the threshold labels (e.g., FTi_FLX, ThC_LEV, etc.) indicated in the SCASM diagram (Fig. 4.1).

4.3 Implementation of SCASM for Intra-Leg Joint Control

Two initial test platforms were constructed to implement the SCASM method for intra-leg joint control. The first was a Single-Leg Test Platform, developed by Brandon Rutter and Brian Taylor, to test the control of a single, 3-DoF leg. The second was a Two-Leg Test Platform, developed by William Lewinger, to test both intra-leg control of two 3-DoF legs and coordination of a two-legged system. Both test platforms were precursors to the six-legged BILL-Ant-a, which uses an implementation nearly identical to that of the Two-Leg Test Platform.

\subsubsection{Single-Leg Test Platform}

The Single-Leg Test Platform (Fig. 4.3) is a 14.3:1 scale 3-DoF model of the right-middle leg of the stick insect Carausius morosus (Rutter et al. 2007). The segment lengths are: $\operatorname{coxa}=0.89$ in $(2.26 \mathrm{~cm})$, femur $=7.59$ in $(19.28 \mathrm{~cm})$, and tibia $=7.44$ in $(18.90 \mathrm{~cm})$, giving the coxa-relative lengths of $1.00,8.58$, and 8.41 , and a 
total leg length of 15.92in $(40.44 \mathrm{~cm})$. Though the animal has more than one DoF at the thoraco-coxal $(\mathrm{ThC})$ joint, only the primary protraction/retraction DoF was used, as in the model of Ekeberg et al. A smooth, low-friction foot, constructed

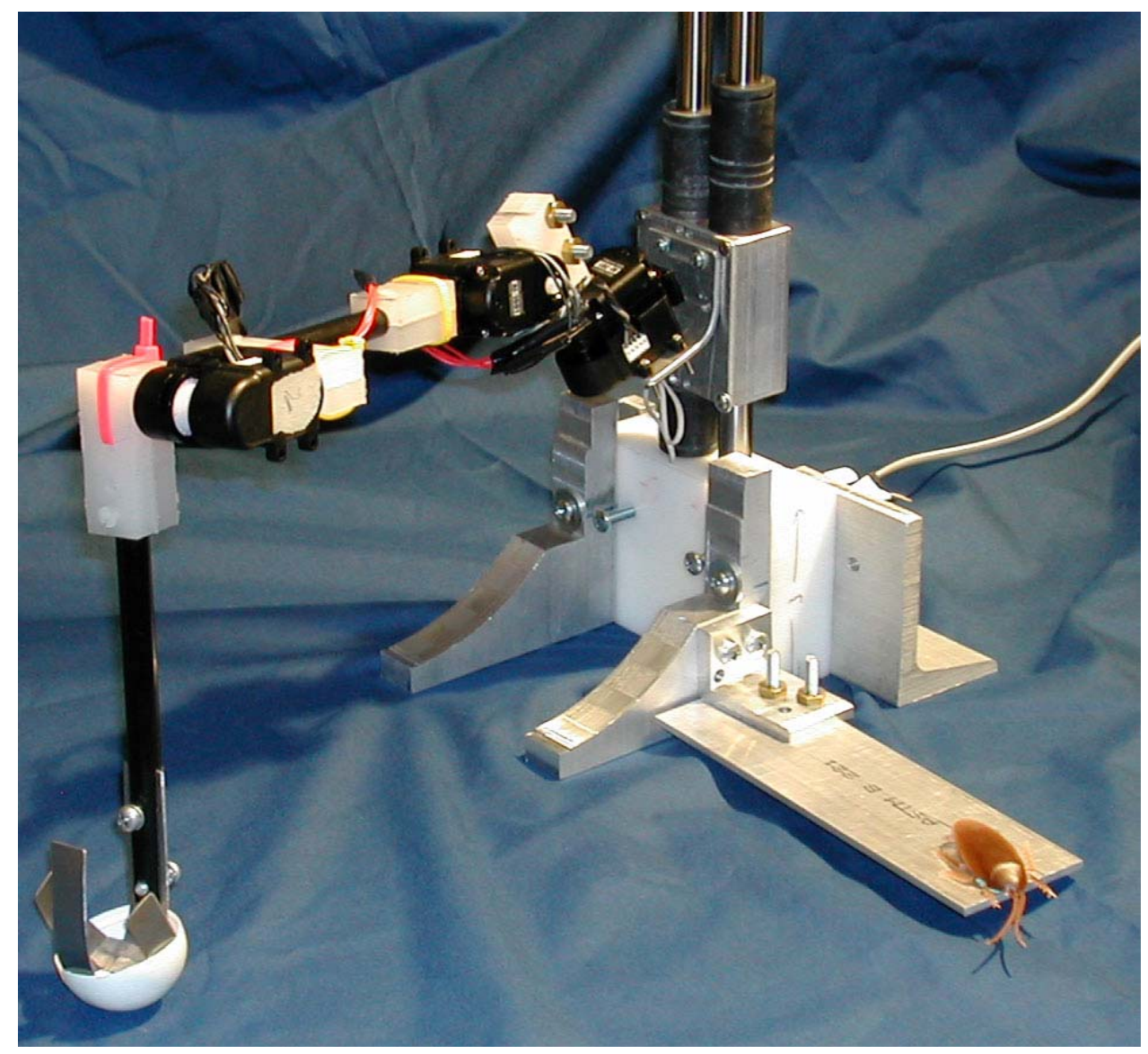

Figure 4.3. Single-Leg Test Platform designed by Brandon Rutter and Brian Taylor.

This is the view from the front. The bar extending to the bottom right indicates the orientation of the thorax in the horizontal plane (Lewinger, Rutter, et al. 2006).

using a half of a table-tennis ball, simulates the frictionless surface used by Ekeberg et al. in their modeling when used on a hard, smooth surface. The joints are actuated using AI-series servo motors from Mega Robotics (Megarobotics Co., Ltd. Seoul, Korea). The ThC and FTi joints use the model AI-701 servo 
while the CTr joint uses the higher-torque model AI-1001. The femur and tibia segments of this prototype were constructed from solid plastic rods (coat-hanger shafts), and these are connected to the motors using adapters made from Delrin ${ }^{\circledR}$ plastic which interface with the standard slide-in connectors of the AI-series servo motors. The motor driving the ThC joint is connected to a body link, which is attached to the base on two vertical sliding rails in order to adjust the modeled body height.

These servos are controlled via an RS-232 serial data line, and provide 8-bit angle and load feedback values to the host controller over the same line. Motor current feedback is available at a resolution of $\sim 18.4 \mathrm{~mA}$, and position command and angle feedback are both at a resolution of 0.654 degrees. Angle data is used directly, but current data is filtered using the following Infinite Impulse Response (IIR) filter (after initialization).

$$
\text { filt_current }=0.05 * \text { current }+0.95 * \text { prev_filt_current }
$$

The system is fast enough (197 Hz motor control loop) that angle feedback is used in conjunction with the servo motors' proportional control to implement a crude, but stable, feed-forward torque control. For a given desired torque output, the servo is commanded to go to some delta of position from the current position; the proportional control used by the servo then generates a torque proportional to this delta. This system generates compliant motion from an electrical motor system with relatively little computational overhead. Control is implemented on a 400 $\mathrm{MHz}$ Pentium-III computer (approximately 400 MIPS) running RT-Linux 
(Yodaiken and Barabanov 1997), which also records all state and sensory data for analysis and allows on-line modification of state transition conditions.

In this model, unlike in the Two-Leg Test Platform and BILL-Ant-a, the current load on the CTr motor is used as a measure of leg load, instead of directly measuring ground contact by a foot-mounted sensor. This is located similarly to some of the load sensing organs in the animal which serve the same purpose (Ekeberg et al. 2004; Hoffmann and Bässler 1982; Akay et al. 2001).

The RT-Linux control system is designed to allow modular addition of "muscle models", dynamic "muscle activation" levels, and the continuous modification of activations and state transition conditions based on higher-level control programs. The "muscle model" allows the joint motor to behave similarly to actual muscle by regulating its torque and speed based on its angular position. This is much like the length-tension properties of biological muscles. Control takes place primarily in two threads. The first of these handles the feed-forward force control and runs as quickly as it can, given computation and serial communication overhead. The second runs at a lower priority and handles the state transition and activation calculations at $100 \mathrm{~Hz}$. Two helper threads convert sensory data into useable form and calculate motor commands based on "muscle activations". These helper threads run whenever they are activated by the two main threads. All control and sensory data are stored in shared memory accessible both from real-time and user space, allowing online modification of the control, and data is logged via FIFO to 
a high-priority user space program which writes all data, including sensor readings, states and "muscle activations", to files.

The state transition thread sets "muscle activations" based on the current state for each muscle. These activations are constant for muscles at the ThC and FTi joints. At the CTr joint the activations are dynamically set based on known activation reflexes, as was done in Ekeberg et al. No explicit "muscle model" was used in these experiments; activations were multiplied by a scaling factor intended to represent a maximum servo delta. The servo delta for a particular state would then be

$$
\text { (agonist - antagonist) * multiplier }
$$

in the direction of the agonist.

\subsubsection{Two-Leg Test Platform}

A second test platform was created using different hobby servo motors for joint actuation (Fig. 4.4). This platform has two 3-DoF legs with an MPI MX-450HP R/C servo motor (Maxx Products, Inc., Lake Zurich, IL, USA) forming each of the three joints. Leg segments lengths are: coxa $=1.35$ in $(3.44 \mathrm{~cm})$, femur $=$ 3.00in $(7.62 \mathrm{~cm})$, and tibia + foot $=4.37 \mathrm{in}(11.11 \mathrm{~cm})$, for coxa-relative segment lengths of $1.00,2.22$, and 3.23. These values are not to scale for the stick insect leg, or any other particular animal. The actuation axes of the thoraco-coxal (ThC) joints are located 4.13 in $(10.48 \mathrm{~cm})$ apart in the frontal plane. This pair of legs was also used for preliminary testing of the BILL-Ant-p hexapod robot (the 
precursor to BILL-Ant-a), but with a different controller (Lewinger 2005; Lewinger, Branicky, and Quinn 2005), as it represents the same leg configuration and thorax mounting geometry as the front legs of the hexapod (and subsequently, BILL-Ant-a as well).

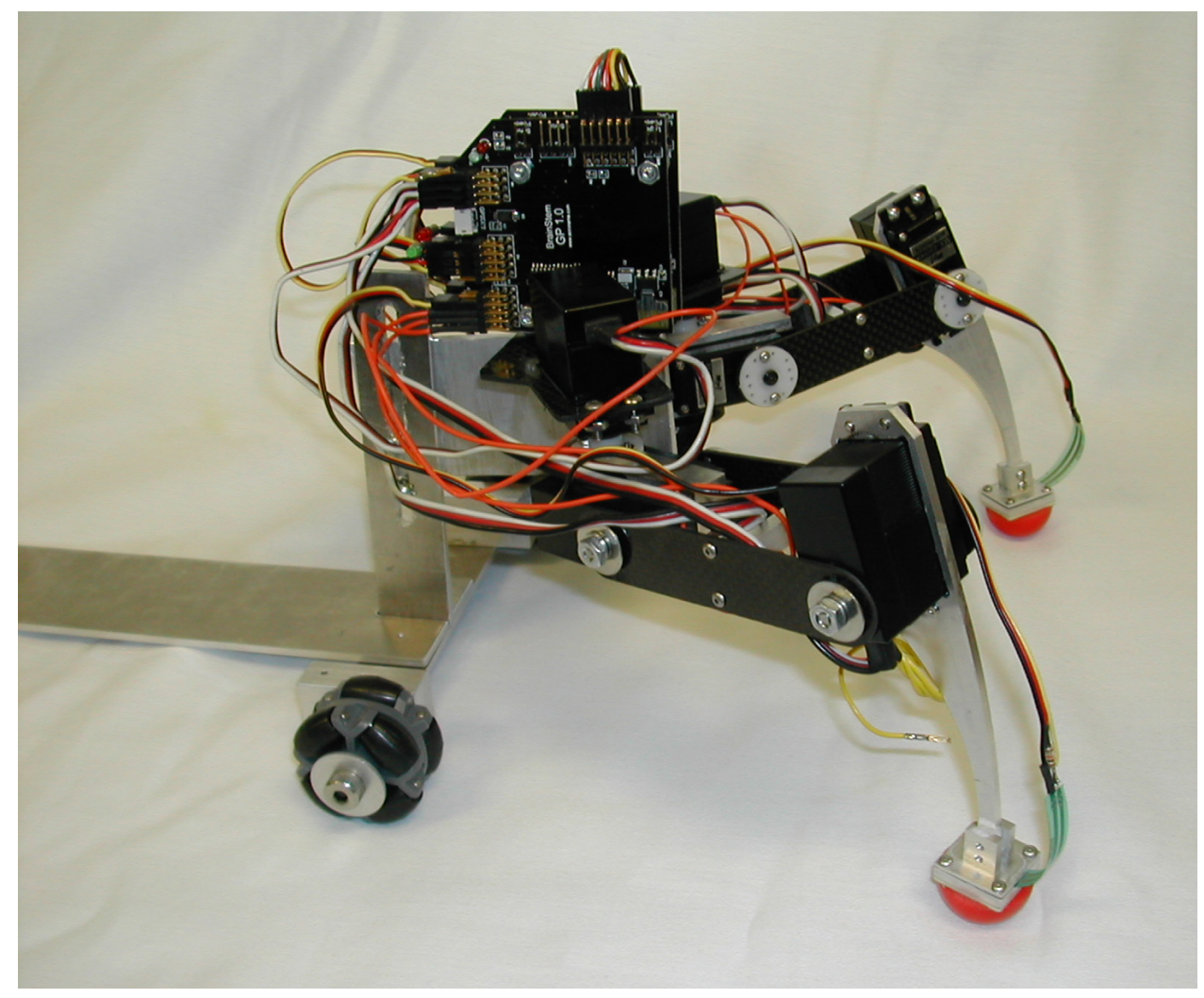

Figure 4.4. Two-Leg Test Platform.

The Two-Leg Test Platform represents the front two legs of the BILL-Ant-a robot, each with its own BrainStem microcontroller.

Hobby servo motors have an internal controller and position feedback loop that move the output shaft to a commanded position. For most R/C servos, that position information is only sensed inside the servo and is not available to the host controller. To provide additional information to leg controllers, signal wires were attached to the position potentiometer located within each servo case and 
connected to the controller via analog-to-digital converter (ADC) ports. This modification allows the leg controller to know both the commanded (internal to the microcontroller) and actual (sensed from the ADC signal) angular position of each joint.

Each leg is controlled by a single PIC-based BrainStem microcontroller (described in Section 3.2.1). The BrainStem is a low computation-capable microcontroller that can only manipulate integer values (no floating point numbers), so more complex methods of intra-leg control, such as inverse kinematics, are impossible. The two controllers used for the test platform are networked to one another via the $\mathrm{I}^{2} \mathrm{C}$ interface bus to create a data connection to a host computer for program initiation and data logging.

Each of the BrainStems is an independent leg controller and commands the three joints of its associated leg. It receives analog inputs from its three $\mathrm{R} / \mathrm{C}$ servo motor joint angles and an additional analog signal from a force sensitive resistor (FSR) located in the base of the foot via ADC ports. For rudimentary inter-leg coordination, a digital signal representing swing and stance is output to the other controller and its corresponding signal is received in a digital input port. While the two microcontrollers are also networked via the $\mathrm{I}^{2} \mathrm{C}$ bus, the digital stance/swing state signals are the only data communicated between the units.

The BrainStem is capable of running up to four small ( $1 \mathrm{~K}$ byte) programs simultaneously by time-slicing of the processor. For the two-leg platform, three programs are running continuously for each leg. One program reads joint angle 
positions and sensed foot load, a second determines the state for each of the three joints based on the SCASM joint coordination rules, and the third actuates the joint servos based on the current state, position, and a simple "muscle model" (Fig. 4.5). The sensor input program, state generating program, and joint actuation programs run at approximately $10 \mathrm{~Hz}, 16 \mathrm{~Hz}$, and $6 \mathrm{~Hz}$, respectively.
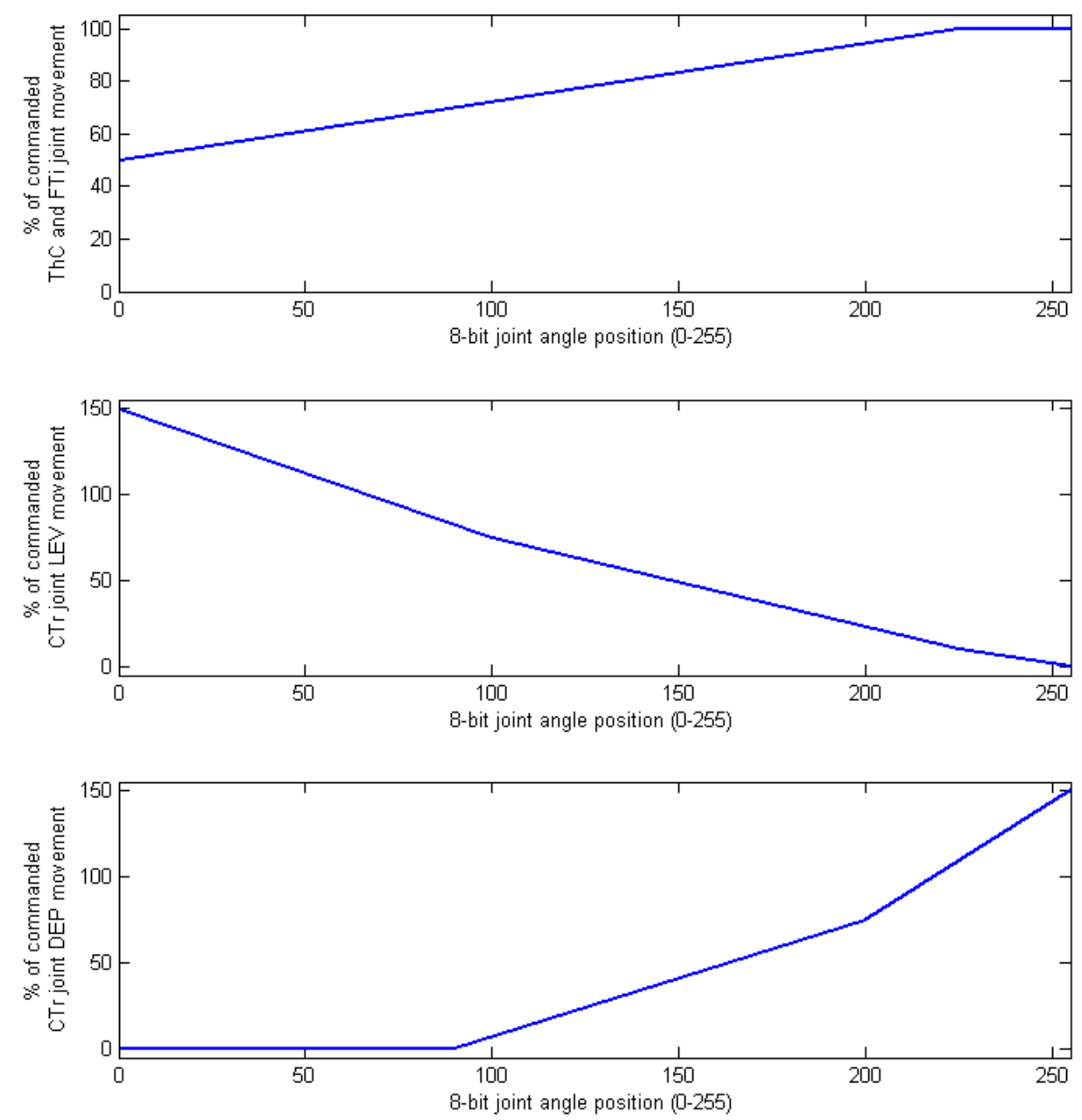

Figure 4.5. Simple "muscle models" used for the Two-Leg Test Platform.

A single "muscle model" is shared for the ThC joint during protraction and retraction and the FTi joint during extension and flexion (top). Separate models are used for the CTr joint during levation (middle) and depression (bottom). 
Each joint is controlled by the basic SCASM control scheme with modified state transition joint angles and "muscle activation" values due to differences in leg geometry and motor properties from the single-leg platform (Table 4.1).

"Muscle activation" levels (agonist and antagonist, each up to 100\%) (Table 4.2) are scaled by a static length-tension "muscle model" and then multiplied by a maximum allowable angular movement to produce the joint actuation commands. This resulting value is then added to the existing joint position to create a new commanded position. The following formula is used for this process:

new_position $=$ old position + MAX_MOVE *

((muscle_model_function(agonist_activation) -

muscle_model_function(antagonist_activation))

\begin{tabular}{|c|c|c|c|}
\hline Joint & State & $\begin{array}{c}\text { Agonist } \\
\text { Activation } \%\end{array}$ & $\begin{array}{c}\text { Antagonist } \\
\text { Activation } \%\end{array}$ \\
\hline ThC & PRO & 70 & 0 \\
\hline & RET & 30 & 10 \\
\hline CTr & LEV & 100 & 0 \\
\hline & DEP & 30 & 10 \\
\hline & DEP* & 5 & 0 \\
\hline FTi & EXT & 10 & 2 \\
\hline & FLX & 05 & 2 \\
\hline
\end{tabular}

Table 4.2. "Muscle activation" values for the Two-Leg Test Platform.

* Special, low-activation case for when the leg is in stance that is used to maintain, rather than increase the body height. 


\subsubsection{BILL-Ant-a}

The third platform to employ the SCASM method of intra-leg joint control, and the focus of this work, is the Biologically-Inspired Legged Locomotion - Ant autonomous (BILL-Ant-a) hexapod robot (Fig. 1.1). An extension of the TwoLeg Test Platform (which is a replication of the front two legs of the hexapod), BILL-Ant-a has six, 3-DoF legs with an MPI MX-450HP hobby servo motor at each of the three leg joints. The leg segment lengths are: coxa $=1.35 \mathrm{in}(3.44 \mathrm{~cm})$, femur $=3.00 \mathrm{in}(7.62 \mathrm{~cm})$, and tibia + foot $=4.37 \mathrm{in}(11.11 \mathrm{~cm})$, for coxa-relative segment lengths of $1.00,2.22$, and 3.23. Additional design specifications on the BILL-Ant-a platform can be found in CHAPTER III.

Each of the six legs in BILL-Ant-a is associated with a single BrainStem microcontroller (Fig. 3.11). For the BILL-Ant-a robot, each BrainStem is responsible for reading analog sensor values from the foot-mounted forcesensitive resistor (FSR), reading the digital status of the lever switch attached to the end of the tibia that is used to sense obstacle collisions, determining joint movement directions based on the SCASM method, and actuating the three servo motors. Three embedded programs, which are similar to those found on the TwoLeg Test Platform, but without the joint angle sensing ability, run on each BrainStem pseudo-simultaneously by sharing clock cycles of the microcontroller (Fig. 4.6).

Figure 4.6. (following page) Flow diagrams of the three programs running on each BrainStem leg controller. 
sensex.tea

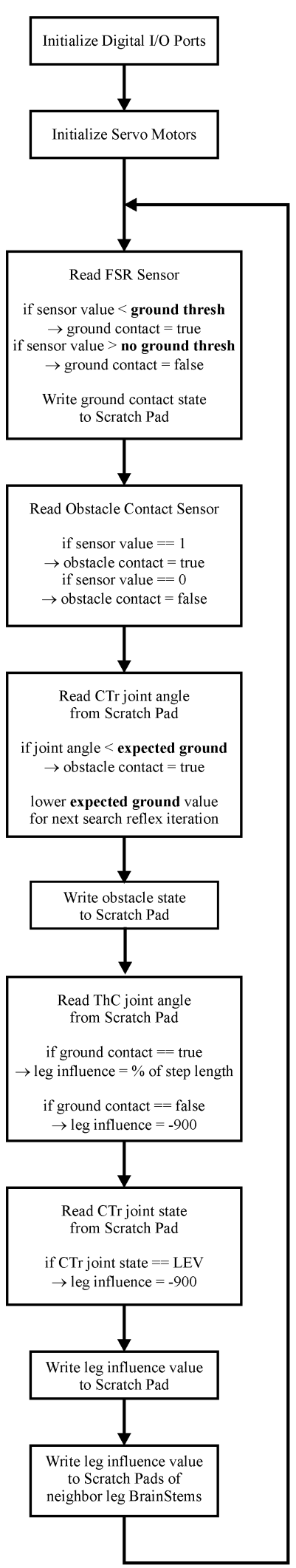

statex.tea

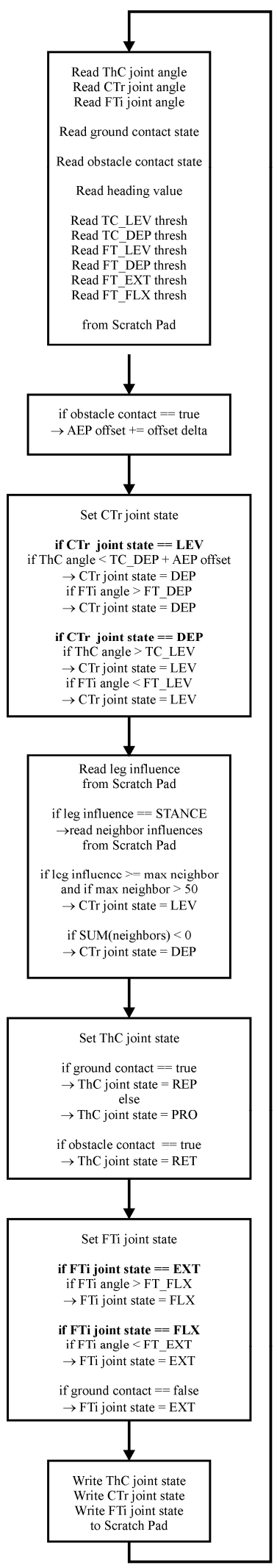

movex.tea

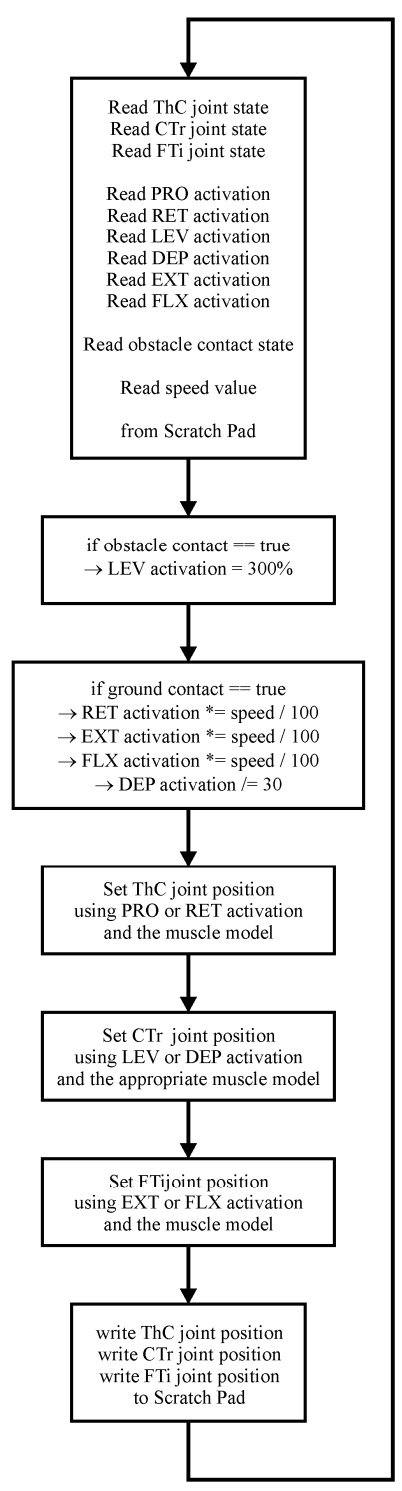


The first of the three programs (sensex. tea; where $x=\{0,1, \ldots, 5\}$ ) (Fig. 4.6) is used to read input values from the foot-mounted FSR and obstacle detection switch, and also to generate a leg coordination influence value to share with orthogonally-neighboring legs. The ground contact status is determined by the value read from the FSR as indicated in Fig. 3.9. Originally, it also read joint angle values from each of the three hobby servo motors; however, once the full gait coordination method was implemented (instead of the basic digital signal used in the Two-Leg Test Platform), the BrainStem was found to be incapable of processing that much information, given its limited processing capabilities. Consequently, joint angles are currently assumed to be at commanded positions, which is a reasonable assumption given the high-gain controller located within each of the hobby servos. The information acquired by the first program is shared with the other two running programs by storing values in the shared memory space of the BrainStem (called the Scratch Pad). This program also initializes the servo motor parameters to establish the operating space as \pm 45 degrees, and configures the digital $\mathrm{I} / \mathrm{O}$ ports.

The second program is statex. tea (where $x=\{0,1, \ldots, 5\})$ (Fig. 4.6). This program obtains information from the Scratch Pad to determine the state for each of the joints: protraction or retraction for the $\mathrm{ThC}$ joint; levation or depression for the CTr joint; and extension or flexion for the FTi joint. Joint states are determined by a combination of factors: joint angle values, ground contact status, obstacle contact status, and the position of the ThC joint and stance/swing phase of the neighboring legs. 


\section{ThC Joint}

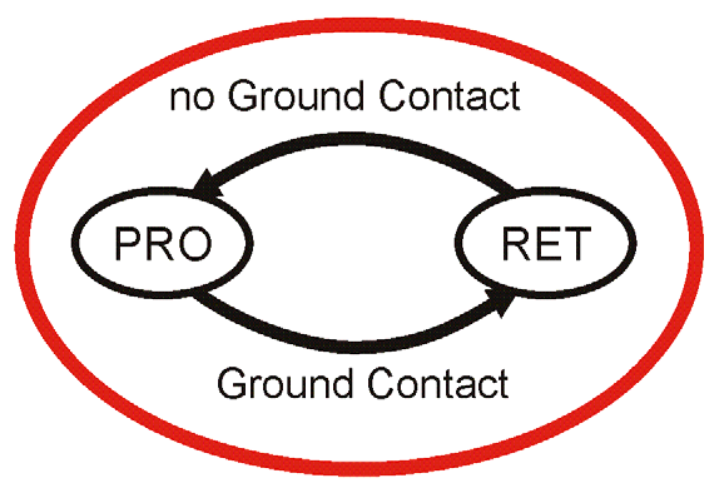

Figure 4.7. ThC joint state diagram.

As its basic level, the thoraco-coxal (ThC) joint state is merely a function of ground contact status (Fig. 4.7), where contact with the ground causes retraction (stance phase) and a lack of ground contact causes protraction (swing phase).

The coxa-trochanter $(\mathrm{CTr})$ joint behavior is more complex and relies on angle information from both of its neighboring joints to determine its state (Fig. 4.8). When in the levation state, a transition to depression occurs if either the ThC joint protracts beyond the TC_DEP threshold or the FTi joint extends beyond the FT_DEP threshold. Either of these conditions will occur during the transition from the swing phase to the pre-stance phase (the second half of the traditionallynamed swing phase).

The addition of the ThC $>$ ThC_DEP condition to induce a transition from levation to depression was added for BILL-Ant-a (and the Two-Leg Test Platform) in order to produce more repeatable stepping motion patterns. Without 


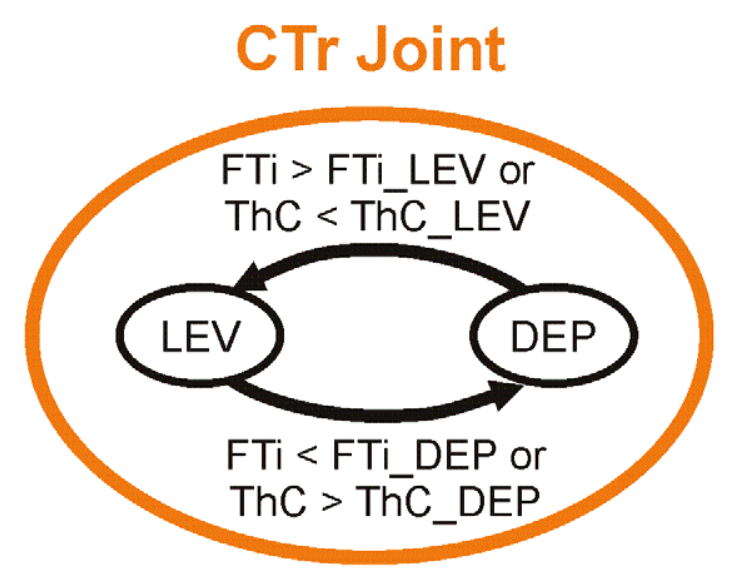

Figure 4.8. CTr joint state diagram.

\section{Note that this joint controller differs from the original SCASM implementation as indicated in Ekeberg et al. (shown in Fig. 4.1) due to the optional condition that $\mathrm{ThC}>\mathrm{ThC} \mathrm{DEP}$ will also cause a transition from LEV to DEP.}

this condition, the FTi joint would over-extend during swing and then flex too aggressively during stance in order to return the FTi joint to a useful angle prior to the onset of swing. The result of this behavior led to the foot path being much skewed from the sagittal plane and caused additional loading on contralateral pairs of FTi joints, which reduced the life of the servo motors. By adding this optional state transition condition (which is now the primary cause for the transition during normal stepping motions), the leg steps in a more regular and repeatable stepping motion and the foot path is parallel to the sagittal plane.

When in the depression state, the joint changes to levation when either the ThC joint retracts past the TC_LEV setpoint or the FTi joint flexes within the FT_LEV angle, which causes the leg to migrate from the stance phase to the pre-swing phase (the second half of the traditionally-named stance phase). 


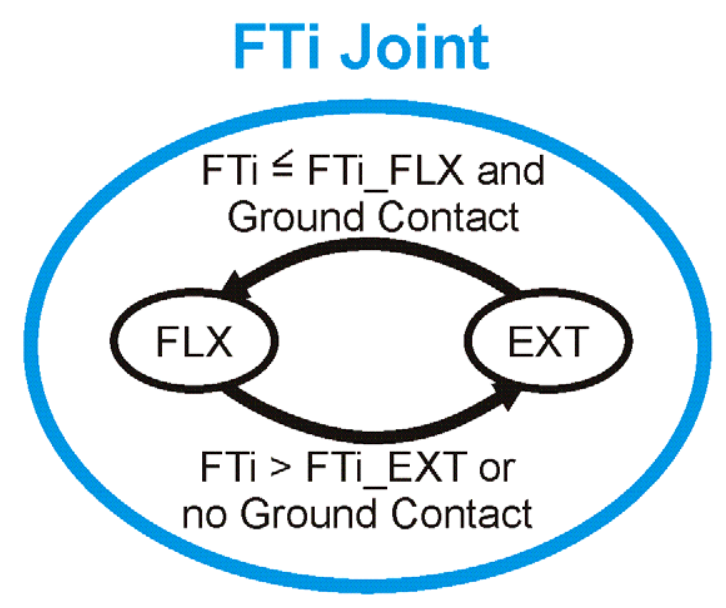

Figure 4.9. ThC joint state diagram.

Finally, the femur-tibia (FTi) joint has its state determined by mostly intra-joint influences (Fig. 4.9). The joint direction changes from flexion to extension when the FTi joint angle moves beyond the FT_EXT threshold. The joint then transitions from extension to flexion when the FTi joint extends past the FT_FLX setpoint. As an over-arching condition similar to the ThC joint, the FTi joint is in extension when ground contact is not detected, independent of the FTi joint angle. This setting of the state is reversed for the rear legs to cause flexion when ground contact is lacking due to the propelling ("pushing") role rear legs perform.

The last program, movex. tea (where $x=\{0,1, \ldots, 5\}$ ) (Fig. 4.6), actuates the joint servo motors based on the joint states, "muscle activation" values, and simple "muscle models". Joint states determined by the statex. tea program are read from the Scratch Pad and determine which direction each of the hobby servos is moved. 
To create smoother joint movements and also to prevent the joints from reaching positions from which they are unable to continue generating stepping motions (as was seen with an early implementation of the Single-Leg Test Platform), simple "muscle models" are used. There is a shared "muscle model" for protraction, retraction, levation, extension, and flexion (Fig. $4.10 \mathrm{a}, \mathrm{b}$, and d); and a separate model for depression (Fig. $4.10 \mathrm{c}$ ). These models differ slightly from those used with the Two-Leg Test Platform, shown in Fig. 4.5. Only two unique models are used here due to the additional amount of code required for BILL-Ant-a over the Two-Leg Test Platform and the BrainStem program size limitation, which eliminated the unique CTr joint levation "muscle model". However, these two models were experimentally shown to be sufficient. While four plots are shown in Fig. 4.10, only two functions are used for the two unique "muscle models". The radically different CTr joint depression "muscle model" is used to promote a simple form of posture control (described further in Section 6.3.5).

Each joint movement command starts with a new base value of 50 (MAX_MOVE in Eq. 4.3). This value is lower than that used for the Two-Leg Test Platform, such that more resolution in "muscle activation" values is available. Also, given the larger program file sizes necessary for BILL-Ant-a, the control loops operate more slowly (approximately $5 \mathrm{~Hz}$ for each program) and so the larger MAX_MOVE value allowed the joints to move well beyond state transition threshold values before they were noticed by the next computation cycle. 
a.

Thoraco-Coxal (ThC) Joint Muscle Model

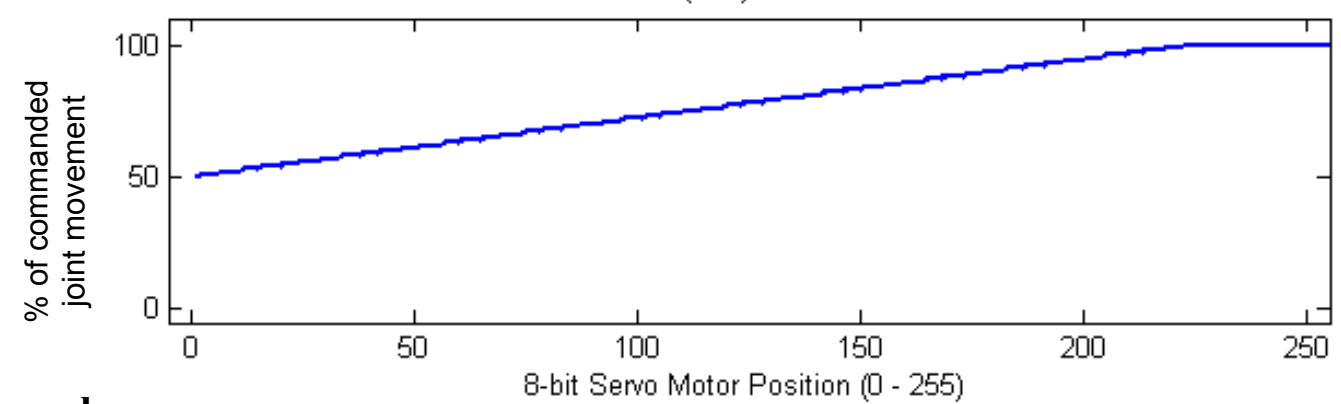

b.

Coxa-Trochanter (CTr) Levation Joint Muscle Model

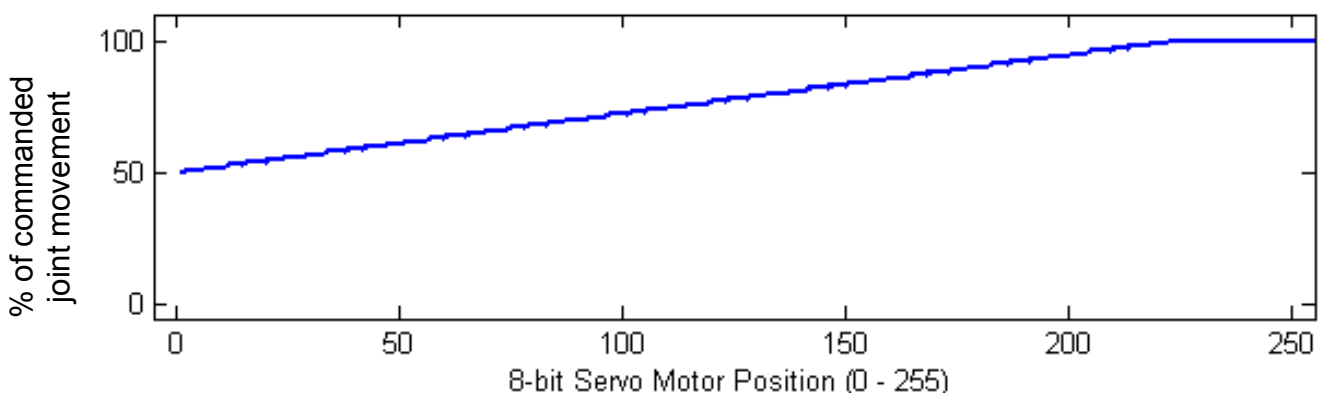

c.

Coxa-Trochanter (CTr) Depression Joint Muscle Model

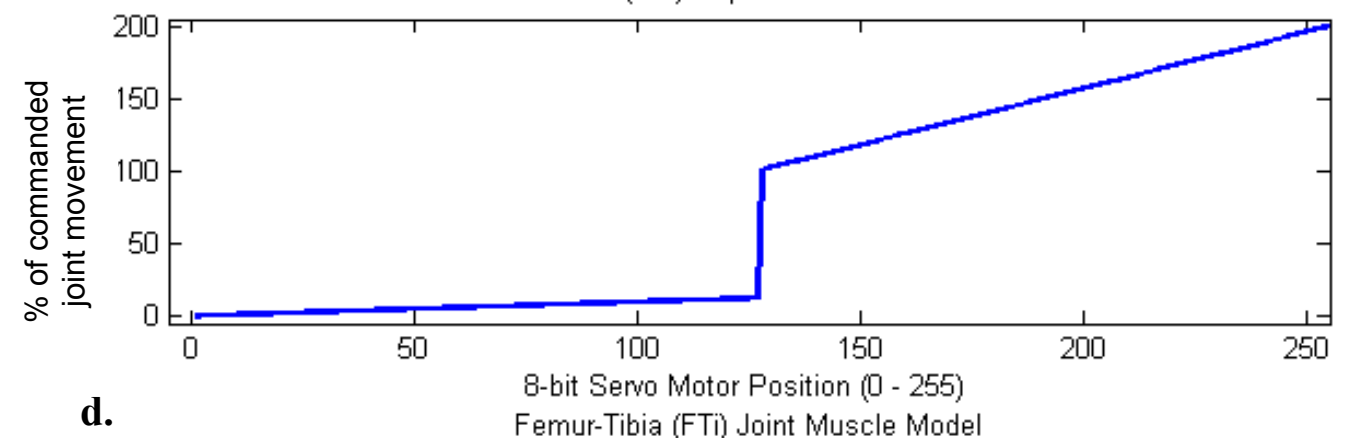

Femur-Tibia (FTi) Joint Muscle Model

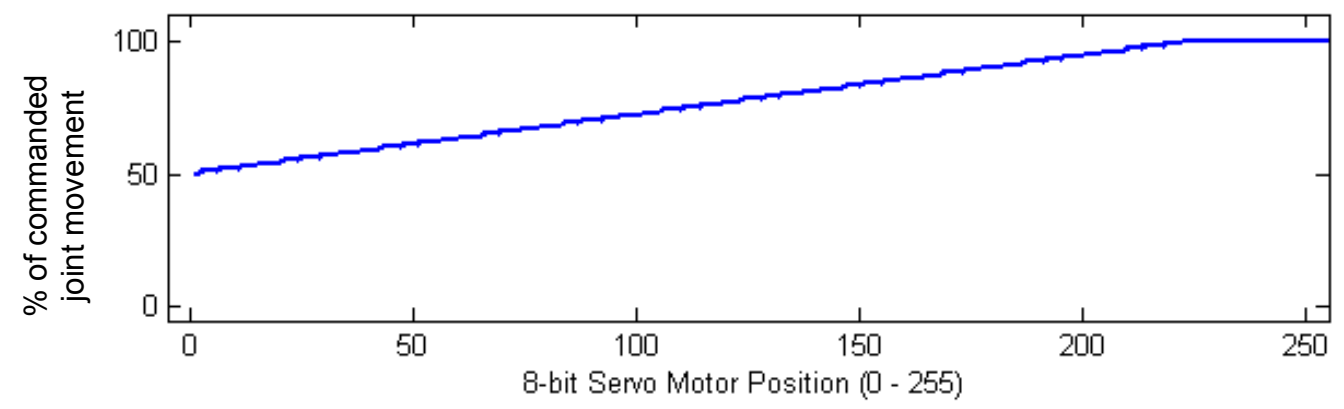

Figure 4.10. Simple "muscle models" used for BILL-Ant-a.

A single "muscle model" is shared for the $\mathrm{ThC}$ joint during protraction and retraction (a), the CTr joint for levation (b), and the FTi joint during extension and flexion (d). A separate model is used for the CTr joint during depression (c). 
Another change from the Two-Leg Test Platform is that the antagonist activation values did not prove to be needed. This is illustrated in Hooper et al. 2009, which showed that the small-mass legs on insects require co-contraction of antagonist muscle pairs, while larger animals, aided by gravitational forces on their largemass limbs, do not require both muscle pairs to be active. Given the scale of BILL-Ant-a, such co-contraction was not needed and the corresponding control software was removed.

\begin{tabular}{|c|c|c|c|c|}
\hline Joint & State & $\begin{array}{c}\text { Front Leg } \\
\text { Activation }\end{array}$ & $\begin{array}{c}\text { Middle Leg } \\
\text { Activation }\end{array}$ & $\begin{array}{c}\text { Rear Leg } \\
\text { Activation }\end{array}$ \\
\hline ThC & PRO & 100 & 100 & 100 \\
\hline & RET & 30 & 30 & 30 \\
\hline CTr & LEV & 50 & 50 & 70 \\
\hline & DEP & 80 & 90 & 100 \\
\hline FTi & EXT & 8 & 8 & 6 \\
\hline & FLX & 18 & 18 & 22 \\
\hline
\end{tabular}

Table 4.3. "Muscle activation" values for BILL-Ant-a.

Similar to the Two-Leg Test Platform calculations, but without the antagonist muscle influence, new joint positions are determined by starting with the MAX_MOVE base value of 50. This is then modified by the "muscle activation" value (Table 4.3) and the appropriate "muscle model" (Fig. 4.10). As an example, the signal sent to the FTi joint during extension when the joint is currently at -90 degrees $\left(127_{2}\right)$ is:

$$
\begin{gathered}
\text { old_pos }+ \text { MAX_MOVE } \cdot((\text { old_pos } \bullet 50 / 225+50) \cdot 6) / 100 / 100=129 \\
127+50 \quad \cdot((\quad 127 \quad \cdot 50 / 225+50) \cdot 6) / 100 / 100=129
\end{gathered}
$$


The resulting commanded position of 129 differs slightly than the calculated value of 129.35 due to the math functions being purely integer operations on the BrainStem. This can also cause slight discrepancies with floating-point math calculations as the results of integer operations are always truncated, and not rounded.

As the behavior of the ThC and FTi joints is rather simple: protract and retract, and extend and flex, respectively, it has been experimentally shown to be sufficient to use simple, identical "muscle models". The CTr joint, however, has a more complex behavior in its roles to both support the body weight and maintain body height, and therefore needed a more complex "muscle model".

During initial testing, all joints and both motor directions shared the same "muscle model" as is currently used for most of the muscles (Fig. $4.10 \mathrm{a}$, b, and d). It was seen, however, that this basic model caused the CTr joint motors to allow the body to sag and ground clearance was quickly reduced to zero. This was due to a lack of posture control. Simple posture control was then implemented in the form of a unique "muscle model" for the CTr joint that provided a form of virtual attractor for when the joint is in depression (DEP_GOAL in Eq. 4.5). The value of DEP_GOAL is unique for each leg due to slight mechanical differences resulting in the same commanded position producing slightly different joint angles. 


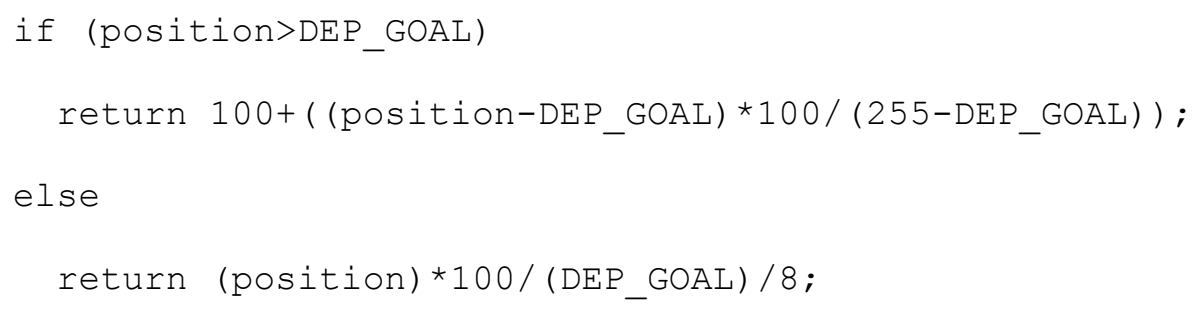

As shown in the CTr "muscle model" in Fig. 4.11c, the joint exerts very little force $(0-12.5 \%)$ when below the depression goal angle. However, when above the desired angle, base-level "muscle activation" is actually multiplied (100 $200 \%$ ). These differences cause the joint to press down vigorously when the body sagged (CTr joint angle $>$ DEP_GOAL) and very lightly when the body is too high $(\mathrm{CTr}$ joint angle $<\mathrm{DEP}$ _GOAL). While not necessarily an ideal form of posture control, this simple fix is both sufficient to minimize body sag and is able to be implemented in the limited program space available.

The combination of the three leg control programs was experimentally shown to generate stepping motions for BILL-Ant-a that resulted in straight-line walking and navigation of slightly irregular terrain heights. That is, terrain heights that did not vary in elevation by more than $+0.5 \mathrm{in} /-1.0 \mathrm{in}(+1.27 \mathrm{~cm} /-2.54 \mathrm{~cm})$.

To facilitate the hand tuning of SCASM parameters, a graphical user interface (GUI) was created that was able to download values into the leg control network (Fig. 4.11). Using the GUI, each of the SCASM state transition thresholds for each of the legs could be modified, even while the robot was walking. Additionally, the "muscle activation" values for each of the three leg pairs (front, middle, and rear) could also be adjusted. On startup, the interface would use default values for the parameters and download them into the BrainStem network. 


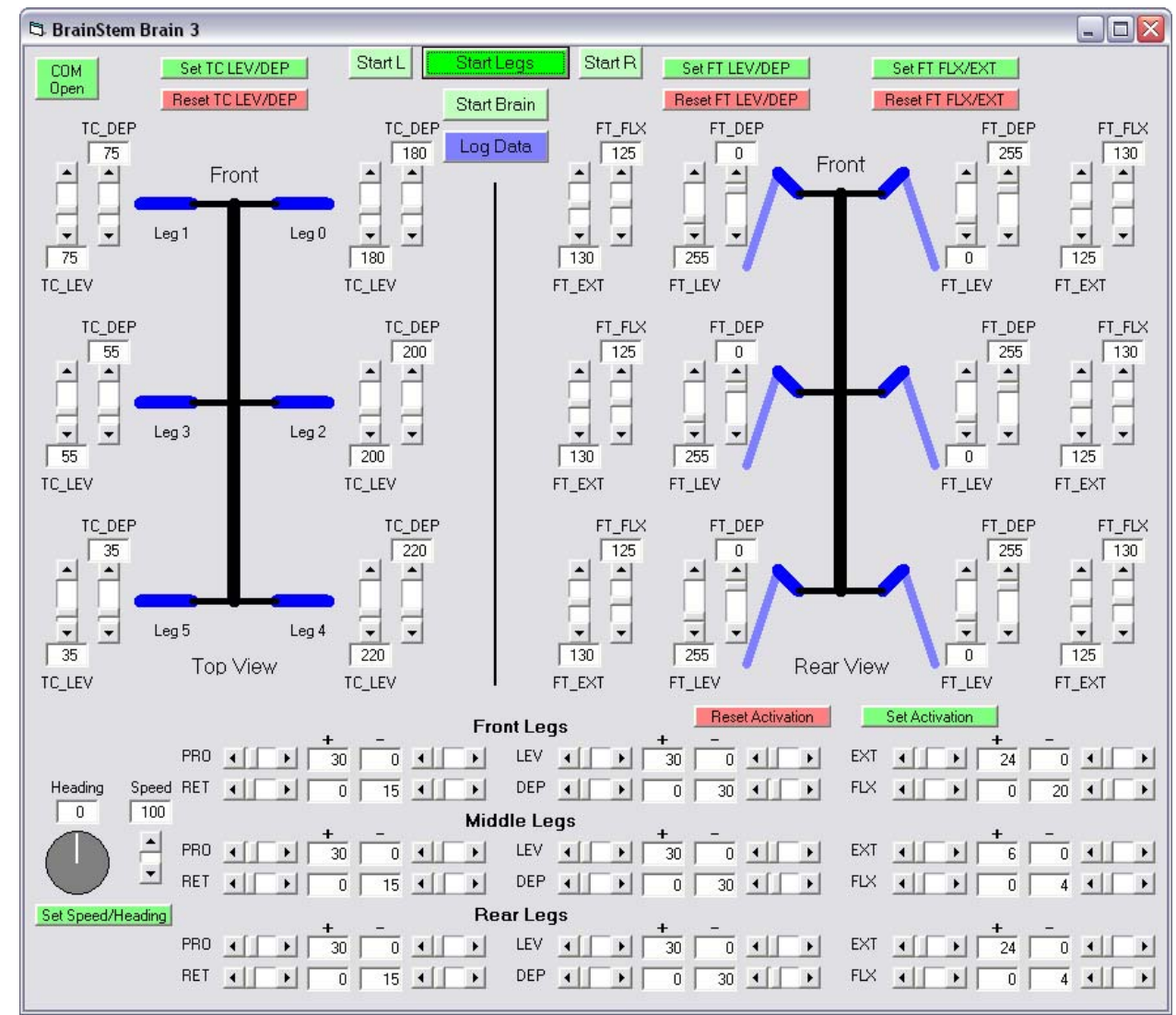

Figure 4.11. BILL-Ant-a graphical user interface (GUI).

This interface was used during the process of hand tuning SCASM parameters. Values could be adjusted and then downloaded to the BrainStem leg controllers.

The Start Legs button was then used to initiate the walking process. At any time, it was possible to update any parameter and download the new value to the appropriate leg(s). The Start Brain button was used to initiate the programs on the "head" BrainStem such that light-based goal seeking would commence.

\section{$4.4 \quad$ Reflexes}

While the implementation of the SCASM method on the BILL-Ant-a hexapod showed robust stepping and straight-line walking on slightly irregular terrain, this 
relatively simple behavior does not make for a very interesting robot. It certainly doesn't justify the use of legs instead of wheels. To remedy this, the ability to overcome more drastic elevation changes and navigate both sparse and highly irregular terrain was added in the form of two reflexes.

These reflex behaviors were provided by the addition of three biologicallyplausible neural pathways in the SCASM control method (Fig. 4.12) (Lewinger and Quinn 2009). These new pathways relay the same type of information, joint angle and joint load, as the existing coupled sensory signals. As with the sensory coupled signals used to generate stepping motions, the new signals act as triggers to modify joint movement directions, thereby changing the stepping motion (Fig. 4.13). When integrated with adjustments to other SCASM parameters, such as the levation "muscle activation", and the TC_DEP state transition threshold setpoint, new obstacle navigating behaviors can be observed.

\subsubsection{Searching Reflex}

The searching reflex, as shown in Fig. 4.13 middle, allows the leg to cross gaps in the terrain. This is useful for navigating sparse environments. The reflex is triggered by the $\mathrm{CTr}$ joint angle depressing lower than expected when seeking ground contact. The Single-Leg Test Platform, Two-Leg Test Platform, and BILL-Ant-a all have the ability to use this reflex behavior. 


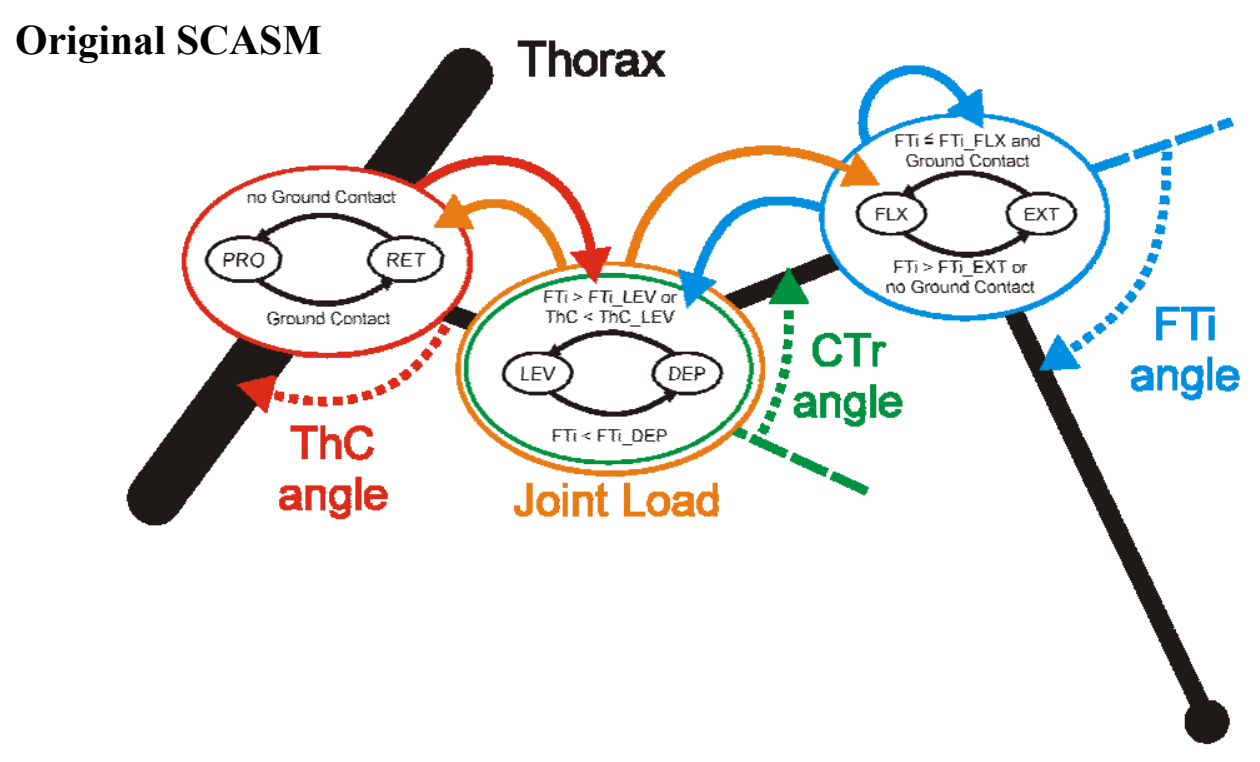

Updated SCASM

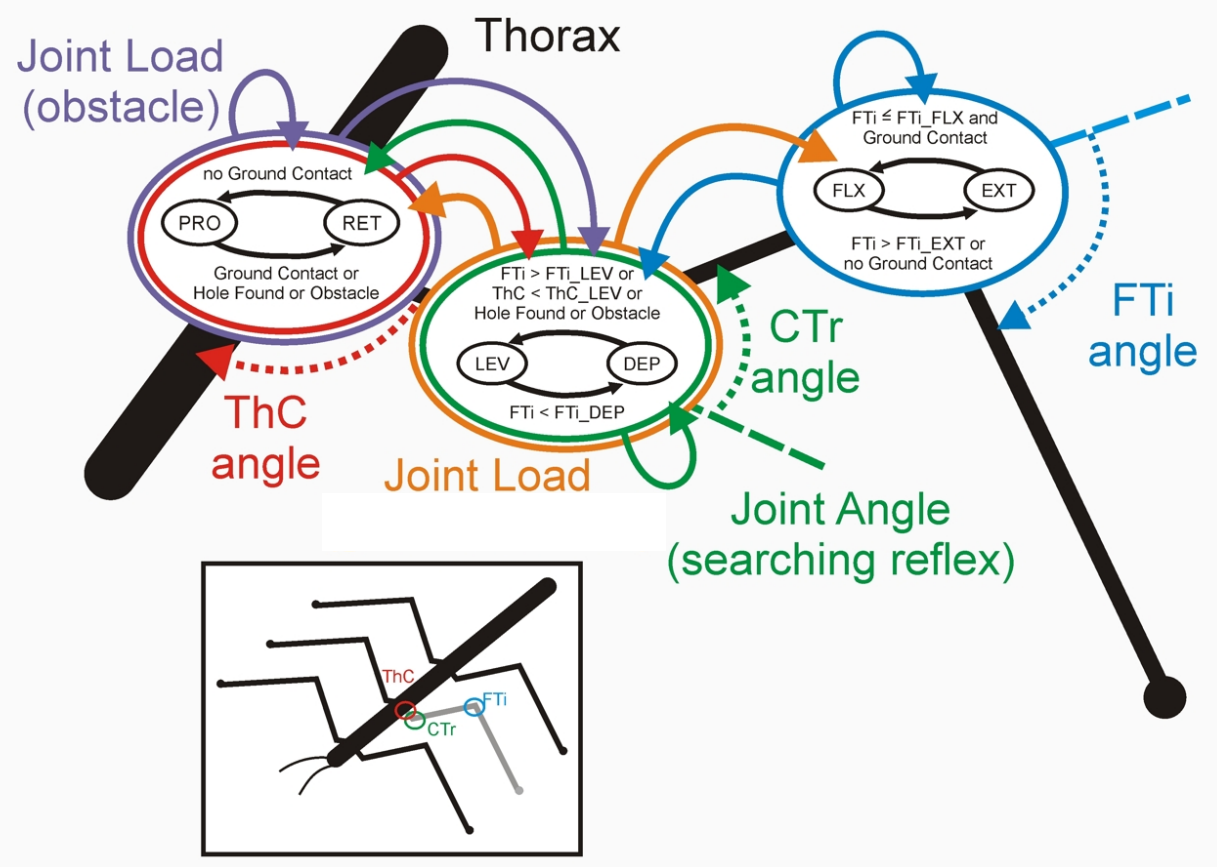

Figure 4.12. Original SCASM diagram (top) and the updated SCASM diagram with the addition of two new biologically-plausible neural pathways (bottom).

The two new pathways examine CTr joint angle to initiate a searching reflex that allows the crossing of sparse terrain, and $\mathrm{ThC}$ joint load to initiate an elevator reflex for navigating past high obstacles. 


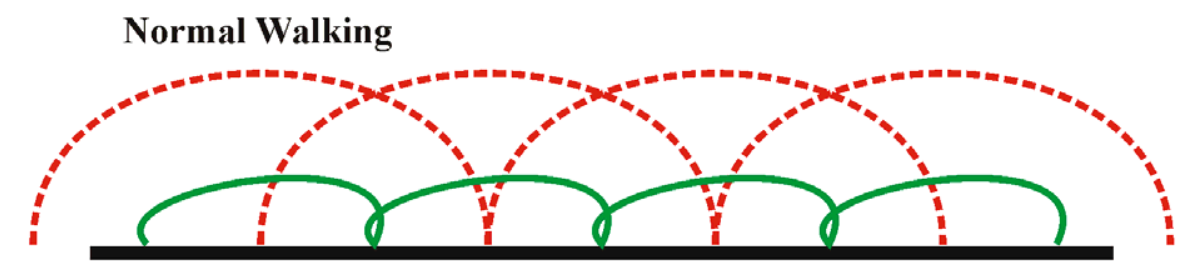

Foot Path

$=-$ Foot Range of Motion
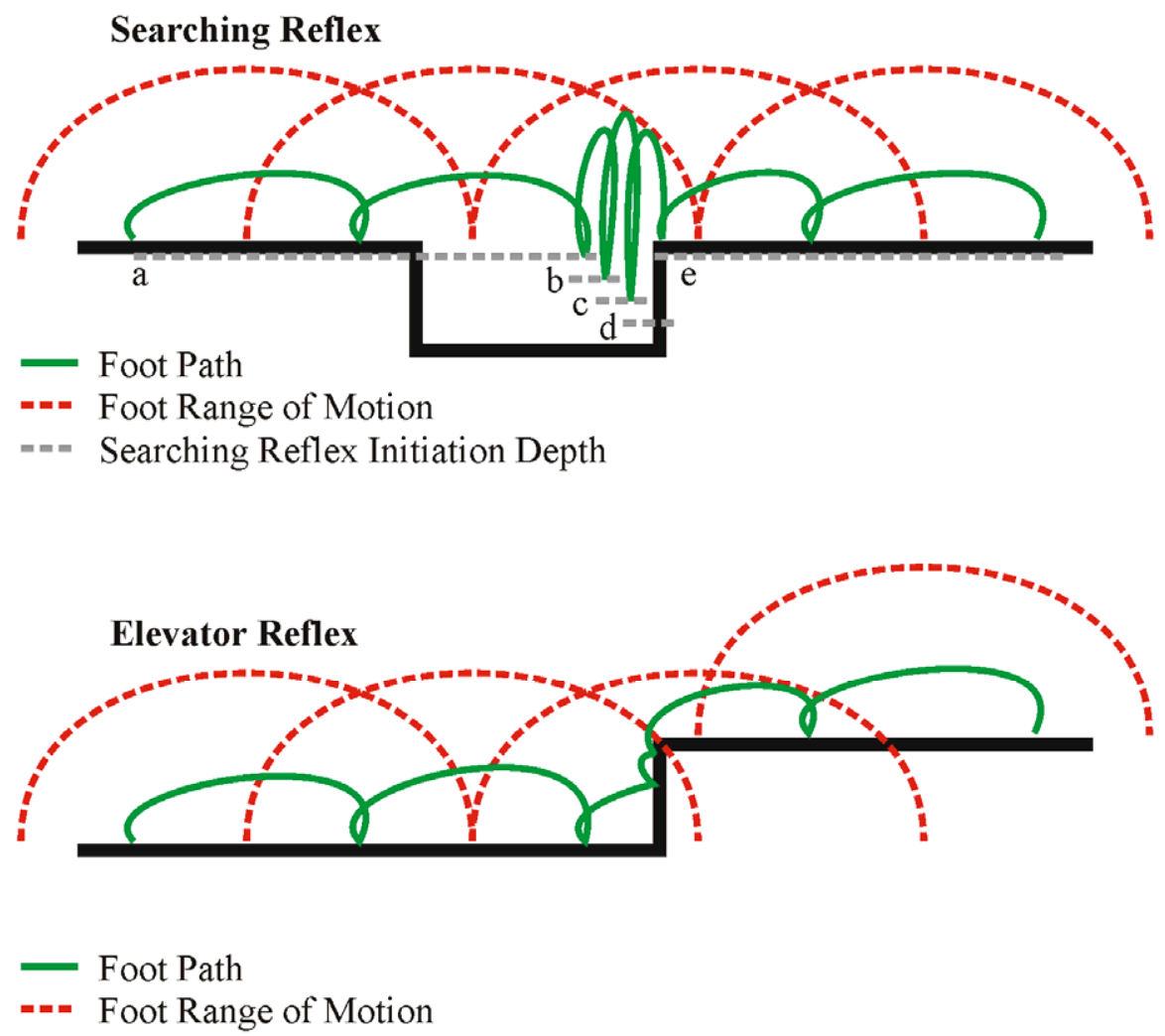

Figure 4.13. Stepping motions for normal walking and the searching and elevator reflex behaviors.

For the Two-Leg Test Platform and BILL-Ant-a, a reflex initiating threshold is defined as being a CTr joint angle slightly below the expected ground contact level. The threshold is part of the sensex. tea program and is implemented as being the $\mathrm{CTr}$ joint angle during normal ground contact less a small offset of $20_{8 \text {-bit }}$ (corresponding to 7.0 degrees of angular rotation, and approximately $0.35 \mathrm{in}$ 
$(0.89 \mathrm{~cm})$ of vertical drop) (represented by the dashed gray line labeled ' $a$ ' in Fig. 4.13, middle). When the CTr joint angle drops below this threshold (Fig. 4.14, ' 3 '), the generic obstacle flag is set and the CTr reflex trigger angle is then lowered by another 7.0deg (0.35in) (represented by the dashed gray line labeled ' $b$ ' in Fig. 4.13, middle). The obstacle flag indicates that either the searching or elevator reflex has been triggered and that a reflex response is required. Its value is stored in the Scratch Pad by the sensex. tea program (Fig. 4.6).

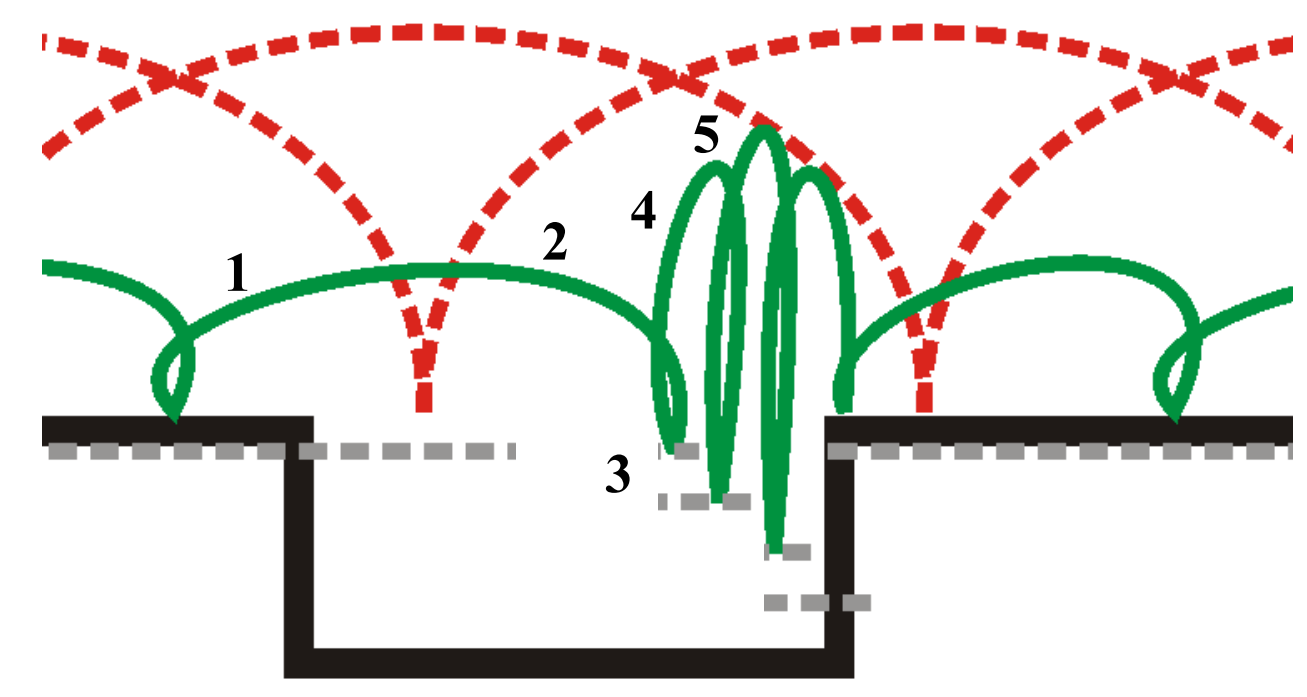

$\begin{array}{lcccc}\text { 1. PRO } & \text { 2. PRO, } & \text { 3. Reflex } & \text { 4. Reflex } & \text { 5. Normal } \\ \text { LEV } & \text { DEP,EXT } & \text { Triggered } & \text { action }- & \text { action }- \\ \text { EXT } & & \text { RET } & \text { PRO } \\ & & & \text { LEV } & \text { DEP } \\ & & & \text { EXT } & \text { EXT }\end{array}$

Figure 4.14. Close-up view of the searching reflex joint state conditions.

The statex. tea program handles the reflex by altering the behavior of the leg joints. When the obstacle flag is detected, the program sets the ThC joint and $\mathrm{CTr}$ joint directions to retraction and levation, respectively (Fig. 4.14, '4'). This will 
cause a change in the current joint states as they should be protracting and depressing as part of the pre-stance action. The FTi joint, in its current state of extension for front and middle legs, and flexion for the rear leg pair, remains unchanged.

Due to the altered joint states, the foot will move backward slightly and upward, as seen in Fig. 4.13, middle. The backward, retraction, action moves the foot away from any potential edge or overhang, in the event that the substrate is resumed near where the foot is currently located. And, to raise the foot in preparation for resumed contact with the ground, the levation action is performed.

In order to overcome the extended depth to which the foot has dropped from its expected ground contact height, the levation action is heightened in the movex. tea program. This program temporarily changes the levation "muscle activation" value to become $300 \%$, regardless of the original value, for a single computation cycle. This exaggerated levation value is sufficient to raise the $\mathrm{CTr}$ joint near the upper limit of its range-of-motion in a single computation cycle.

These alterations to the stepping motion occur for one calculation cycle only. After which time, normal joint state behaviors resume (Fig. 4.14, '5'). In the event that the next pre-stance cycle does not result in contact with the ground by the time the CTr joint drops to the new reflex trigger threshold, the process will repeat with the CTr reflex trigger threshold angle being lowered during each iteration (represented by dashed gray lines labeled ' $c$ ' and ' $d$ ' in Fig. 4.13, middle). 
Once ground contact is re-discovered, normal stepping resumes. The CTr joint angle reflex trigger threshold is reset to become 7.0deg lower than the current angle when the ground is detected. In the event that the substrate is not found by the time the CTr joint has reached its lower range-of-motion, the process will repeat indefinitely, with the robot stuck at the edge of the cliff. There is currently no behavior in BILL-Ant-a that can identify this condition and elect to reverse its course in search of a new path. This limitation is due to a lack of available program space in the BrainStem modules to accommodate such a behavior.

\subsubsection{Elevator Reflex}

The elevator reflex is employed to navigate over tall obstacles in the robot's path (Fig. 4.14 bottom). This is useful for climbing stairs or simply stepping over minor obstacles in the way.

BILL-Ant-a has a nominal step height of about $0.5 \mathrm{in}(1.27 \mathrm{~cm})$. This height emerges from the set of SCASM parameters that orchestrate the stepping motion. When the foot collides with an obstacle having a height taller than the nominal step height, the elevator reflex will be initiated. As described in the updated SCASM diagram in Fig. 4.13, this could be detected by sensing the increased load in the ThC joint during its protraction effort. However, since BILL-Ant-a does not have load sensing in its joints, an obstacle contact switch was mounted on the tibia and its lever is formed to lie in front of the foot (Fig. 3.10). The switch lever is positioned such that its tip is near the foot/substrate level when the leg is 
stepping. This allows obstacles with heights of just over $0.5 \mathrm{in}(1.27 \mathrm{~cm})$ to activate the switch.

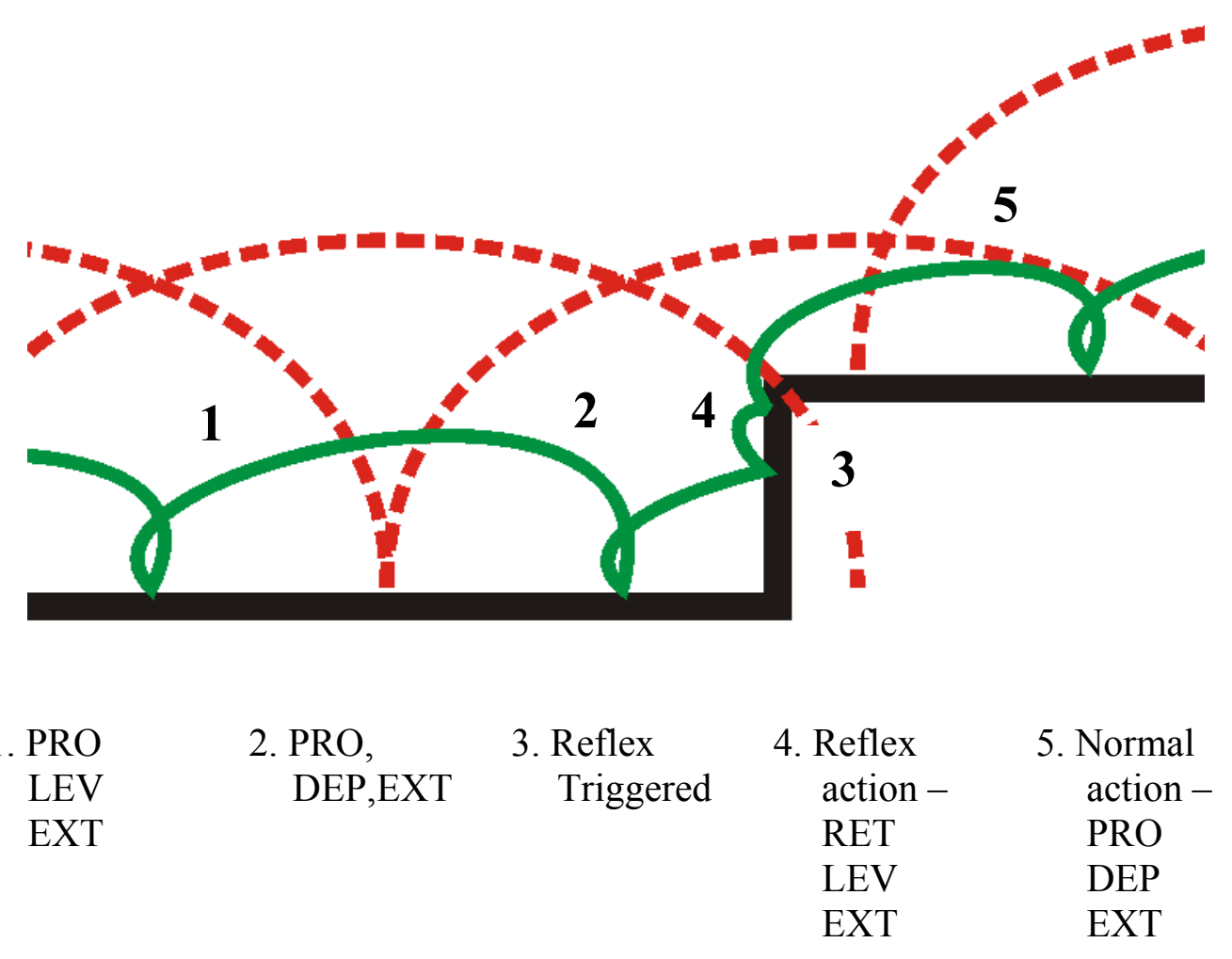

Figure 4.15 Close-up view of the elevator reflex joint state conditions.

When the obstacle contact switch is depressed by a collision with an obstruction, the elevator reflex is triggered (Fig. 4.15, ' 3 '). Similar to the searching reflex, the status of the obstacle contact switch is monitored by the sensex. tea program, which sets the obstacle flag when the switch is actuated.

When the statex.tea program reads the obstacle flag, two sets of actions occur: the $\mathrm{ThC}$ joint and $\mathrm{CTr}$ joint movement directions are set to retraction and levation (the same as for the searching reflex) (Fig. 4.15, '4'), and the TC_DEP 
state transition threshold value is advanced by up to $30_{2}$ (equal to 10.5 degrees of ThC joint motion and approximately $0.98 \mathrm{in}(2.49 \mathrm{~cm})$ of forward foot motion).

The changes in joint state produce the same effects as that of the searching reflex, causing the leg to retract and levate. In this case, the retraction action is required to disengage the foot and obstacle contact switch from the face of the impinging obstruction. The levation action is used to raise the foot height in order to step over the obstacle. As before, the movex. tea program amplifies the levation action by temporarily raising the "muscle activation" level to $300 \%$.

Once the obstacle contact switch is no longer depressed, the stepping behavior continues with the leg protracting in its swing phase and the TC_DEP state transition threshold value advanced slightly forward (Fig. 4.15, '5'). This causes the leg to take a longer step in the forward direction, in an attempt to step beyond the obstacle. If the obstacle is again encountered, and the obstacle contact switch is depressed, the reflex process repeats.

When ground contact is re-established, the TC_DEP state transition threshold value is reset to its nominal ThC joint angle position, and normal stepping motions resume.

As with the searching reflex, in the event that the obstacle is not cleared by the time the $\mathrm{C} \operatorname{Tr}$ joint reaches its upper range-of-motion, providing the foot with a clearance height of approximately $2.25 \mathrm{in}(5.72 \mathrm{~cm})$, the leg will continue to repeatedly impact the obstruction with a cycle of retraction and protraction of the 
ThC joint. Due to a lack of available BrainStem program space, no behavior was provided that would allow BILL-Ant-a to retreat and choose an alternate path.

\subsection{Modulation of SCASM}

The addition of the two reflexes improves the navigation ability of BILL-Ant-a, and justifies the implementation of legs instead of wheels. However, the need to turn is also a requirement of a practical mobile vehicle. And, the ability to perform some form of autonomous behavior, such as goal seeking, would greatly enhance the usefulness of the robot.

The seventh microcontroller, designated as the "head", interfaces with two infrared (IR) phototransistors that form a stereoscopic light sensing unit, which is used to find light-based goals. A single hobby servo motor acting as a neck is used to pan the sensor pair left and right by \pm 45 degrees (Fig. 3.10, right). The "head" reads the IR sensor values via two analog-to-digital (ADC) ports. Left and right sensor values are compared to one another and the neck servo motor is actuated to equalize the two values. The heading value created by the position of the neck motor when the two light levels are equal is then used to modulate the SCASM state transition threshold values and the "muscle activation" levels via descending commands as from higher centers in insects (Wessnitzer and Webb 2006). This modulation causes BILL-Ant-a to change its stepping motion patterns, which results in the robot changing its course and walk toward a light source. 
As is seen in cockroaches ( $\mathrm{Mu}$ and Ritzmann 2005), each of the six feet adopts a different foot path in order to create turning movements of the body (Fig. 4.16). During free walking and turning (Fig. 4.16, left), the foot paths deviated from the sagittal plane by up to 90 degrees. For tethered walking and turning (Fig. 4.16, right), however, foot path deviations from the sagittal plane seem to be limited to approximately 45 degrees. Although the foot path headings for free walking and turning are outside of the reachable space for BILL-Ant-a, the behavior exhibited by the freely moving animal also represents the insect performing load bearing actions. As BILL-Ant-a is also required to bear its own weight, the free-moving foot path headings were used as a basis for the robot. However, given the limited leg ranges-of-motion for the robot, the foot path headings shown in Fig. 4.15, left were divided by two. This yielded foot path headings approximately between $10.1 \mathrm{deg}-45 \mathrm{deg}$.

Testing of the SCASM modulation method was conducted by creating a basic kinematic simulation with MATLAB. The simulator was designed to replicate a Two-Leg Test Platform leg, using the segment lengths, ranges of joint motion, and fixed body height. The SCASM control method as implemented on the TwoLeg Test Platform (including the "muscle models") was used as a basis for the simulation; however, since this was not a full dynamical model, some minor adjustments in "muscle activations" were needed. The simulation was used to determine if: a) variations in joint state change setpoints and "muscle activation" 

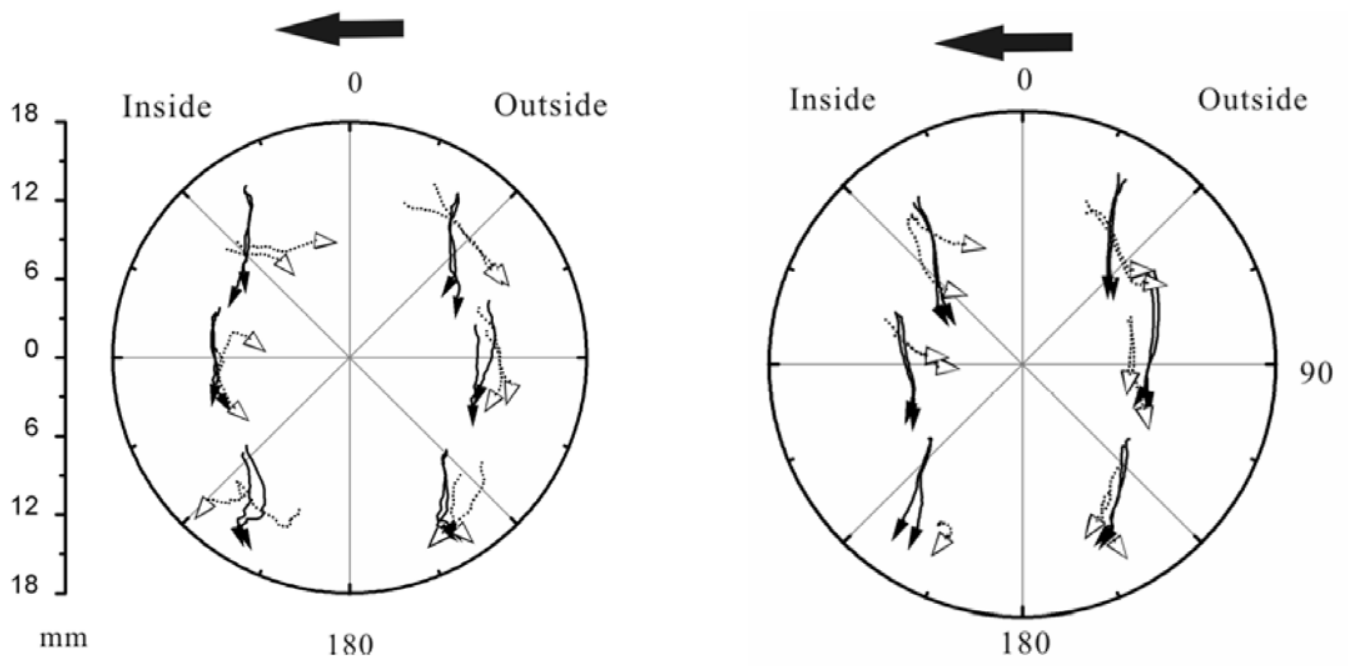

Figure 4.16. Cockroach foot paths during turning.

(left) Cockroach foot paths during straight-line walking (solid line) and left turning (dashed line) while free-walking. (right) Cockroach foot paths during straight-line walking (solid line) and left turning (dashed line) during tethered walking (Mu and Ritzmann 2005).

levels could cause foot path deviations of up \pm 45 degrees from the sagittal plane; and b) the setpoint variations could be expressed in simple first-order, linear equations. The desire for simple equations was necessary for implementation using the BrainStem microcontroller with its limited mathematical capabilities.

Initially, the simulator was configured to create a straight-line walking stepping motion similar to that seen in the Two-Leg Test Platform. This confirmed that the necessary changes in the simulated "muscle activations" produced basic stepping motions akin to the robot (Fig. 4.17). To be considered "acceptable", the foot positions were limited to $\pm 1 \mathrm{~cm}$ from the desired foot path heading (roughly the width of the foot itself). 

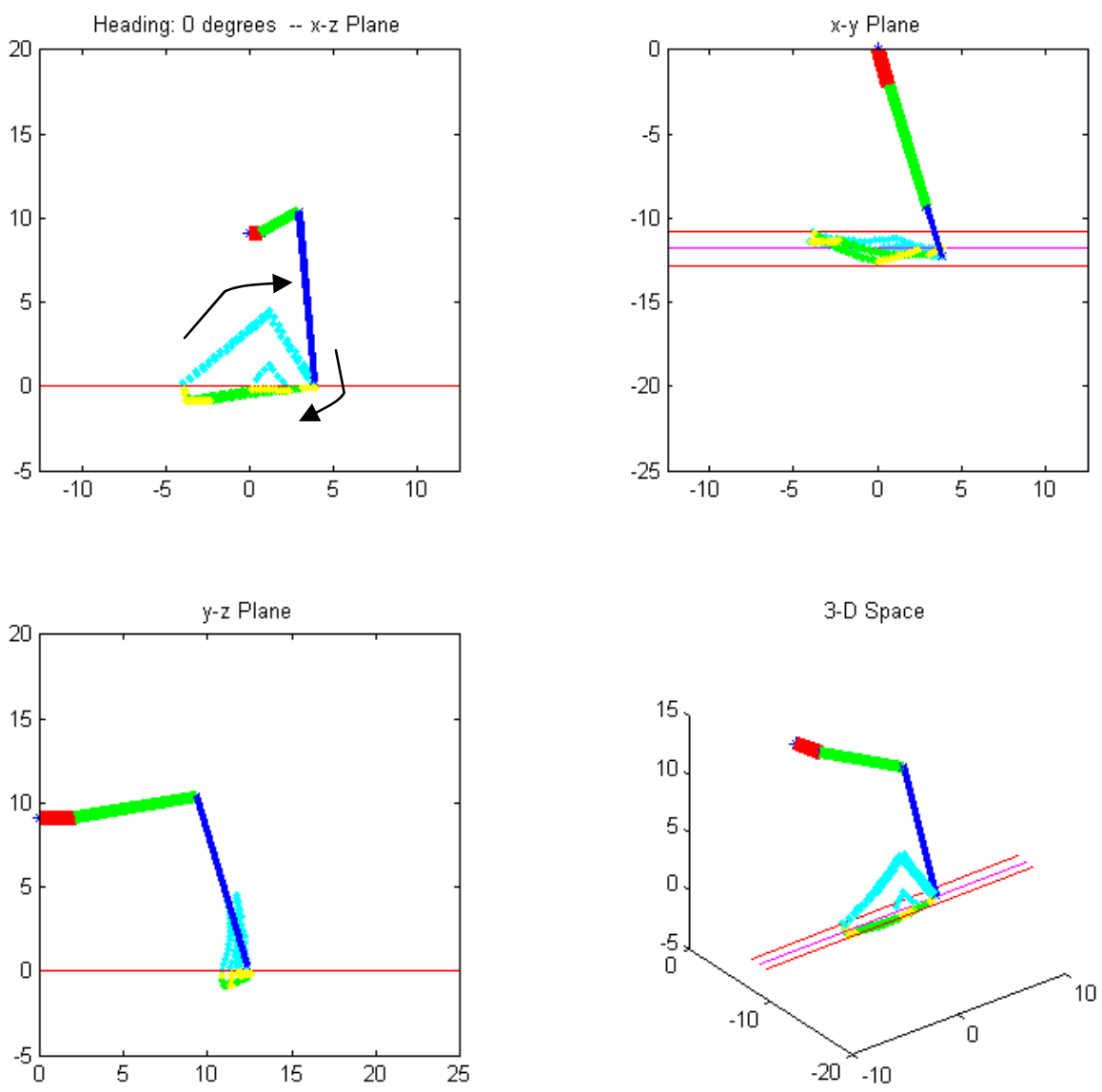

Figure 4.17. Simulated right leg foot path along the sagittal plane.

Four views of the same foot path for a middle-right leg during two step cycles along the sagittal plane. Cyan markers indicate when the foot is in swing phase; yellow markers indicate that the FTi joint is in extension; green markers represent the FTi joint in flexion.

Next, joint state transition thresholds and "muscle activation" levels were manually adjusted in order to achieve a foot path that was -45 degrees from the sagittal plane while maintaining the same step length and step height that was seen in the straight-walking simulation. This proved to be a daunting task, given that variations on up to twelve parameters were being performed. Instead, an iterative, brute-force, process was programmed into the simulator. A more 
sophisticated method, such as a genetic algorithm, was not used since the search space was somewhat limited in size,. This process varied each of the twelve parameters, in turn, and evaluated the resulting stepping motion for the criteria of step length, step height, and deviation from the desired foot path heading (-45deg for this test). Eventually, sets of viable parameters were found that fit the criteria and produced an acceptable stepping motion (Fig. 4.18).

Following this success, testing was conducted to find parameters that would lead to a step path that was +45 degrees to the sagittal plane. This was conducted in a similar manner as the $-45 \mathrm{deg}$ test with an automated search of the parameter space, and the results were compared to the same criteria of step length, step height, and deviation from the desired $+45 \mathrm{deg}$ heading. The simulated stepping motions for this heading are shown in Fig. 4.19.

The resulting parameter sets for +45 deg walking were found to be different than those of the $-45 \mathrm{deg}$ walking test: the FTi extension "muscle activation" values were lower and flexion activation values were higher than those of their $-45 \mathrm{deg}$ counterparts; and the FT_FLX and FT_EXT state transition threshold values were both higher, with FT_FLX being nearly double the angle selected for the $-45 \mathrm{deg}$ trial. 

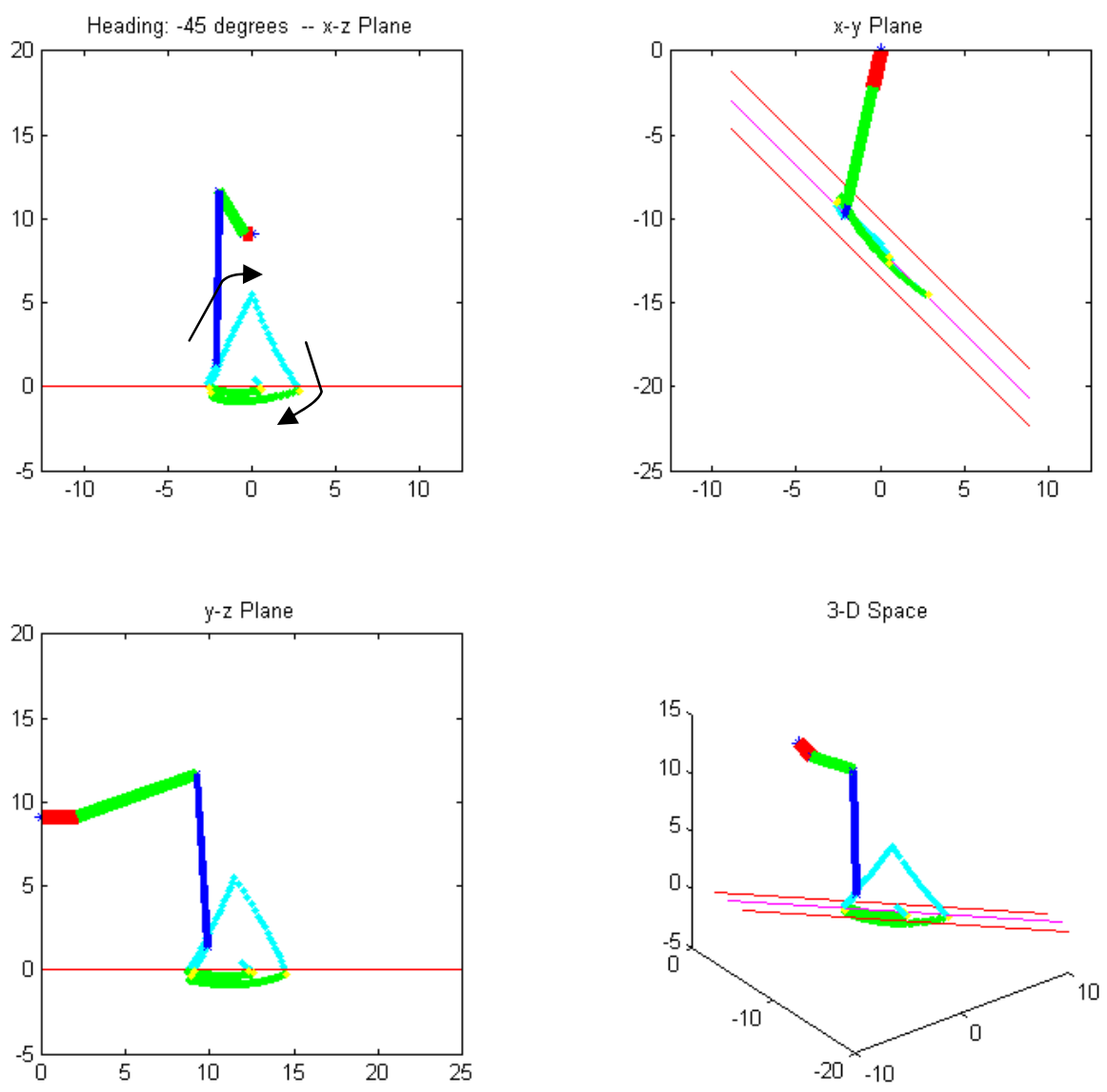

Figure 4.18. Simulated right leg foot path at $\mathbf{- 4 5 d e g}$ to the sagittal plane.

Four views of the same foot path for a middle-right leg during one step cycle at an angle of $\mathbf{- 4 5 d e g}$ with the sagittal plane. Cyan markers indicate when the leg is in swing phase; green markers indicate that the FTi joint in flexion for most of the stance phase; yellow markers represent that the FTi joint is briefly in extension.

These differences were expected due to the asymmetry of the leg's reachable space about the sagittal plane and the alteration in roles of the leg during inside and outside turning. For the simulated right-side leg, the $-45 \mathrm{deg}$ test represents inside turning, where the leg has a "pulling" role. During the $+45 \mathrm{deg}$ test, the leg is an outside leg and has a "pushing" role. These changes in leg function explain the differences in the resulting parameter sets. 

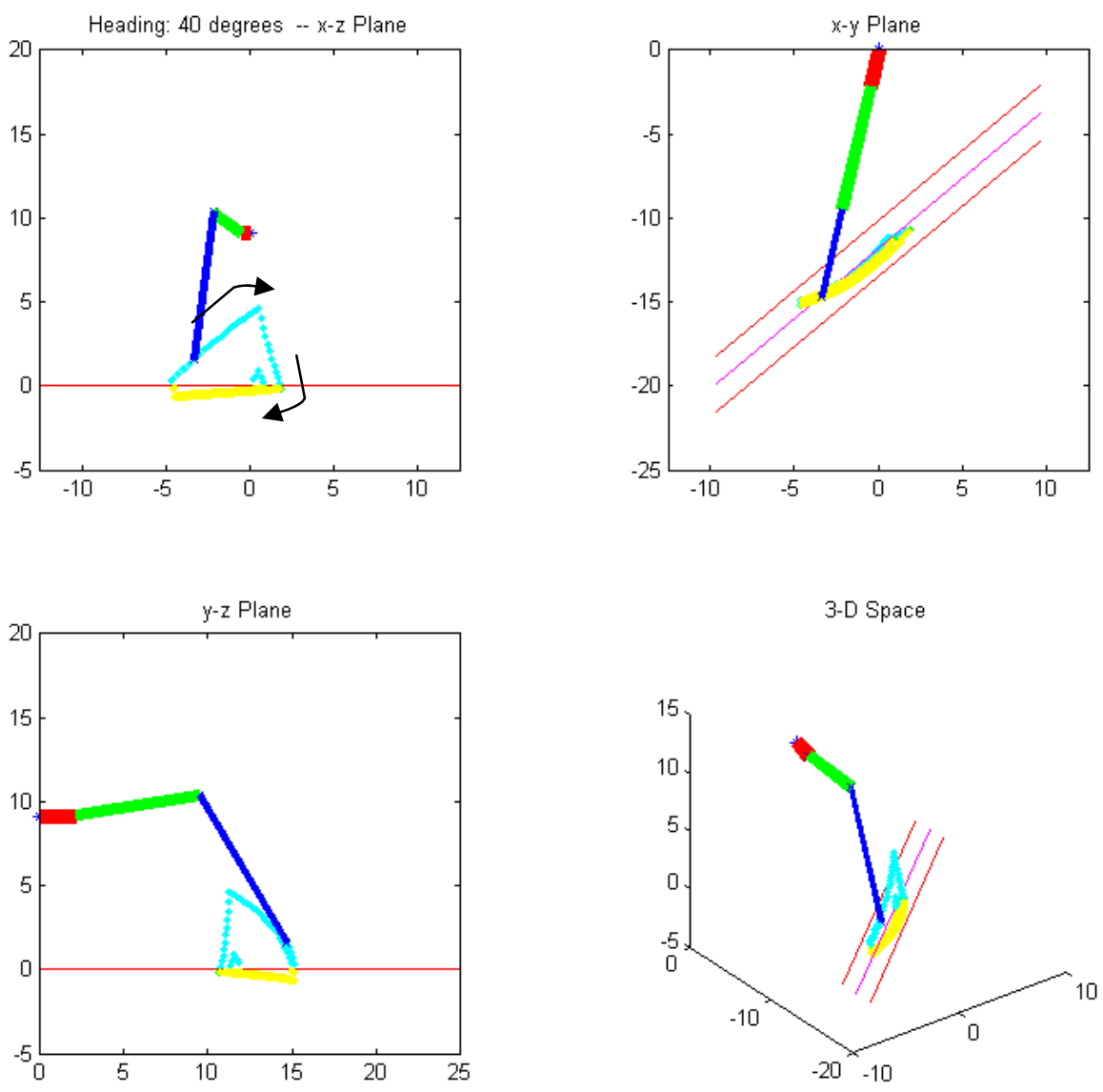

Figure 4.19. Simulated right leg foot path at $+45 \mathrm{deg}$ to the sagittal plane.

Four views of the same foot path for a middle-right leg during one step cycle at an angle of $+45 \mathrm{deg}$ with the sagittal plane. Cyan markers indicate when

the leg is in swing phase; yellow markers indicate that the FTi joint in extension for the entire stance phase. No green markers are seen as the FTi joint did not enter flexion.

Once these two trials showed that the simulated leg could reach foot path headings of $+/-45$ degrees, the next step was to determine if varying the parameters between straight-line walking levels and the $+/-45 \mathrm{deg}$ extremes could be done with a set of linear equations. This was performed to discover if a set of basic equations could achieve foot path headings for the angles between 0deg and $+45 \mathrm{deg}$, and $-45 \mathrm{deg}$ to $0 \mathrm{deg}$. 
For these tests, the simulator was configured to sequentially vary the foot path heading from $0 \mathrm{deg}$ to $+45 \mathrm{deg}$ in 5 degree increments. The same criteria of step length, step height, and deviation from the heading were tested as with the fixed heading angle trials. For each iteration, the SCASM parameters were adjusted linearly from straight-walking levels to the newly discovered values that produced +45deg stepping. The stepping motions were found to exhibit less-straight foot paths (more of a zig-zag pattern due to oscillations between FTi joint extension and flexion), but the paths were still within the $\pm 1 \mathrm{~cm}$ deviation for each of the intermediate headings. Similar iterations were performed for the 0deg to $-45 \mathrm{deg}$ range and showed similar results of less-straight, but still acceptable foot paths.

Several differences in the leg behavior between positive and negative headings were observed during the full set of trials. The first was that the behavior of the FTi joint reversed between the two extremes. Foot paths in the sagittal plane use a combination of FTi flexion (green markers) and extension (yellow markers) while the ThC joint is in retraction (Fig. 4.17). However, this behavior is altered when foot paths are outside of the sagittal plane, such that flexion (green markers) is used nearly exclusively during $\mathrm{ThC}$ retraction when the foot path angle is negative (Fig. 4.18), while extension (yellow markers) is used exclusively when the foot path angle is positive (Fig. 4.19). This altered behavior in FTi direction is reversed for legs on the left side of the robot: FTi extension occurs during ThC retraction for foot path deviation angles $>0 \mathrm{deg}$ and FTi flexion occurs for negative foot path angles. 


\begin{tabular}{|c|c|c|}
\hline \multicolumn{3}{|c|}{ Simulated foot path headings of $\leq 0 \mathrm{deg}$ with the sagittal plane } \\
\hline Altered Value & Joint State & Equation \\
\hline ThC protractor & ThC PRO & $($ base activation $)+\frac{35 \cdot \text { foot path heading }}{45 \mathrm{deg}}$ \\
\hline ThC retractor & ThC RET & $($ base activation $)+\frac{15 \cdot \text { foot path heading }}{45 \mathrm{deg}}$ \\
\hline CTr levator & CTr LEV & base activation (no change) \\
\hline CTr depressor & CTr DEP & base activation (no change) \\
\hline FTi extensor & FTi EXT & (base activation) $-\frac{24 \cdot \text { foot path heading }}{45 \mathrm{deg}}$ \\
\hline FTi flexor & FTi FLX & (base activation) $-\frac{24 \cdot \text { foot path heading }}{45 \mathrm{deg}}$ \\
\hline ThC_LEV & $\mathrm{CTr} \mathrm{DEP} \rightarrow \mathrm{LEV}$ & $($ base setpoint $)+\frac{5 \cdot \text { foot path heading }}{45 \mathrm{deg}}$ \\
\hline ThC_DEP & $\mathrm{CTr} \mathrm{LEV} \rightarrow \mathrm{DEP}$ & (base setpoint) $-\frac{15 \cdot \text { foot path heading }}{45 \mathrm{deg}}$ \\
\hline FTi_LEV & $\mathrm{CTr} \mathrm{DEP} \rightarrow \mathrm{LEV}$ & base threshold setpoint (no change) \\
\hline FTi_DEP & $\mathrm{CTr} \mathrm{LEV} \rightarrow \mathrm{DEP}$ & base threshold setpoint (no change) \\
\hline FTi_EXT & FTi FLX $\rightarrow$ EXT & (base setpoint $)+\frac{35 \cdot \text { foot path heading }}{45 \mathrm{deg}}$ \\
\hline FTi_FLX & $\mathrm{FTi} \mathrm{EXT} \rightarrow \mathrm{FLX}$ & base threshold setpoint (no change) \\
\hline
\end{tabular}

Table 4.4. Simulator SCASM value equations for foot path headings of $\leq 0 \mathrm{deg}$ (stepping to the right).

\section{Variations in SCASM state change threshold values and "muscle activation" values required to alter the foot path to headings $\leq 0 \mathrm{deg}$ with the sagittal plane.}

During the simulations, the baseline joint state transition values and "muscle activations" were changed linearly with the desired deviation angle from the sagittal plane. These changes, shown in Table 4.4 and Table 4.5 , showed that deviation angles of $\pm 45 \mathrm{deg}$ were possible with two simple sets equations for each of the altered values: one set for foot path headings $\leq 0 \mathrm{deg}$ from the sagittal plane (Table 4.4), and another set for headings $>0$ deg (Table 4.5). 


\begin{tabular}{|c|c|c|}
\hline \multicolumn{2}{|c|}{ Simulated foot path headings of $>$ odeg with the sagittal plane } \\
\hline Altered Value & Joint State & Equation \\
\hline ThC protractor & ThC PRO & (base activation) $-\frac{35 \cdot \text { foot path heading }}{45 \text { deg }}$ \\
\hline ThC retractor & ThC RET & (base activation) $-\frac{15 \cdot \text { foot path heading }}{45 \text { deg }}$ \\
\hline CTr levator & CTr LEV & base activation (no change) \\
\hline CTr depressor & CTr DEP & base activation (no change) \\
\hline FTi extensor & FTi EXT & (base activation) $+\frac{4 \cdot \text { foot path heading }}{45 \text { deg }}$ \\
\hline FTi flexor & FTi FLX & (base activation) $+\frac{44 \cdot \text { foot path heading }}{45 \text { deg }}$ \\
\hline \hline ThC_LEV & CTr DEP $\rightarrow$ LEV & (base setpoint) $-\frac{6 \cdot \text { foot path heading }}{45 \text { deg }}$ \\
\hline ThC_DEP & CTr LEV $\rightarrow$ DEP & (base setpoint) $+\frac{9 \cdot \text { foot path heading }}{45 \text { deg }}$ \\
\hline FTi_LEV & CTr DEP $\rightarrow$ LEV & base threshold setpoint (no change) \\
\hline FTi_DEP & CTr LEV $\rightarrow$ DEP & base threshold setpoint (no change) \\
\hline FTi_EXT & FTi FLX $\rightarrow$ EXT & base threshold setpoint (no change) \\
\hline FTi_FLX & FTi EXT $\rightarrow$ FLX & base threshold setpoint (no change) \\
\hline
\end{tabular}

Table 4.5. Simulator SCASM value equations for foot path headings of $>0 d e g$ (stepping to the left).

Variations in SCASM state change threshold values and "muscle activation" values required to alter the foot path to headings $>0 \mathrm{deg}$ with the sagittal plane. Note that the equation changes are not pure sign reversals from those for headings of $\leq \mathrm{deg}$; this is due to range-of-motion limitations for the leg joints.

It was also found that not all of the setpoints needed to change in order to achieve the desired foot path. The simulations showed that not all setpoint and "muscle activation" values were required to change in order to adopt a new foot path, which is depicted in Table 4.4 and Table 4.5. 
When these heading-altering methods were implemented on the Two-Leg Test Platform, minor modifications were needed (as was expected, given that the simulator was not a fully dynamical environment with an accurate model of the physical system). While the general trends observed in the simulations were maintained, such as the limited subset of parameters that actually required changing, and the relative magnitudes of change (for some parameters), the implemented numerical values were adjusted to achieve good results for the physical platform.

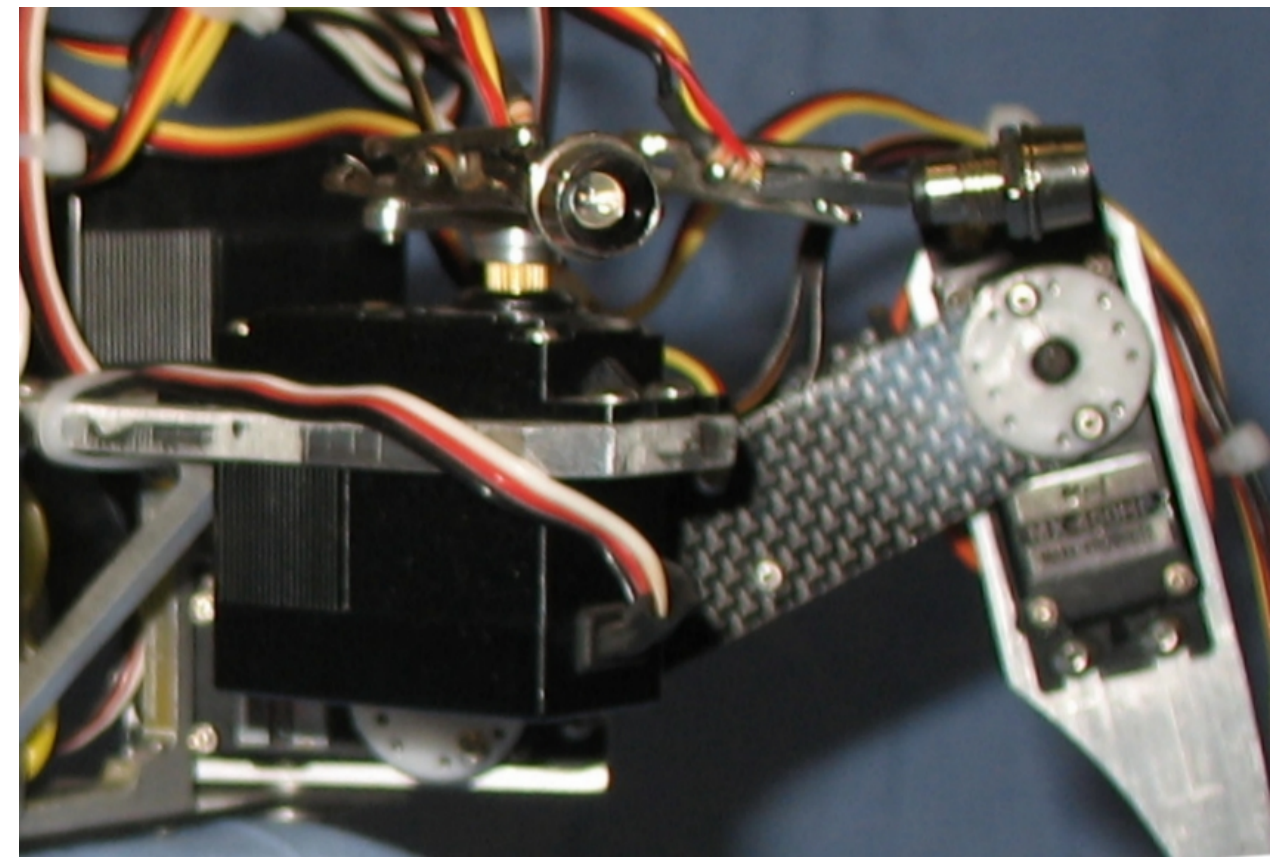

Figure 4.20. Early neck and stereo phototransistor assembly.

A pair of phototransistors, secured via alligator clips and wired to the "neck" BrainStem microcontroller via a resistor network, mounted to the neck servo. The servo motor is capable of panning motions, left and right.

Physical experiments were conducted with a variant of the Two-Leg Test Platform that added a third BrainStem microcontroller to act as the "head", along 
with the neck and stereo phototransistor assembly from BILL-Ant-a (Fig. 4.20). The new BrainStem performed the phototropic seeking behavior by actuating the neck to balance the sensed light levels and then modulated the SCASM parameters for the two legs in order to walk toward the light source. These trials showed that modulation of the SCASM parameters with simple, linear equations was sufficient to affect the heading of the robot such that it could walk toward external, sensed goals.

When the SCASM modulation method was implemented on BILL-Ant-a, further hand tuning of the modulation equation sets was required, due to differences in the allowable leg ranges-of-motion between the hexapod and the Two-Leg Test Platform. Another adjustment made during the BILL-Ant-a implementation was varying the amount by which the leg values were modulated. For the Two-Leg Test Platform, there was a one-to-one mapping from the neck angle to the foot path modification, such that when the neck found a light-based goal at $-45 \mathrm{deg}$, both legs had their foot paths modulated to achieve -45 deg headings. For BILLAnt-a, each leg has a unique heading assigned, based on the experimental results of Mu and Ritzmann for free walking cockroach turning (Fig. 4.21).

As is seen in Fig. 4.21, each leg during the turning action moves in a different heading. The front inside leg of the insect moves at roughly +90deg, while the front outside leg moves at about one third of that $(+30 \mathrm{deg})$. As these foot path headings were impossible with BILL-Ant-a, relative headings were used based on the maximum possible $+/-45 \mathrm{deg}$ values. So, the front inside leg of the robot 
adopts a heading of $+45 \mathrm{deg}$, while the front outside leg is assigned a heading of $+15 \mathrm{deg}$. The remaining legs of the robot have headings that have been similarly scaled by half of that seen in the insect.

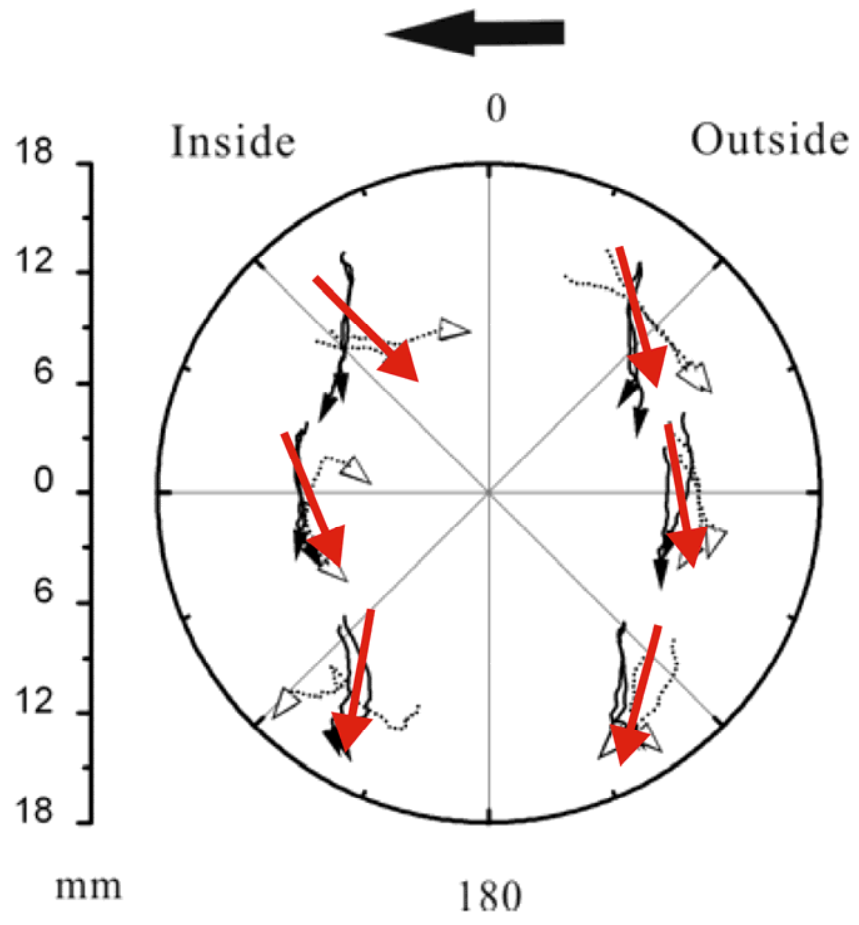

Figure 4.21. Cockroach foot paths during free turning.

Cockroach foot paths during straight-line walking(solid line) and left turning (dashed line) during free-walking (Mu and Ritzmann 2005). Red arrows indicate foot path directions implemented for BILL-Ant-a in response to a $+45 d$ eg neck heading.

The resulting adjustments to the SCASM parameter modulation equations as implemented for BILL-Ant-a are shown in Table 4.6 and Table 4.7. 


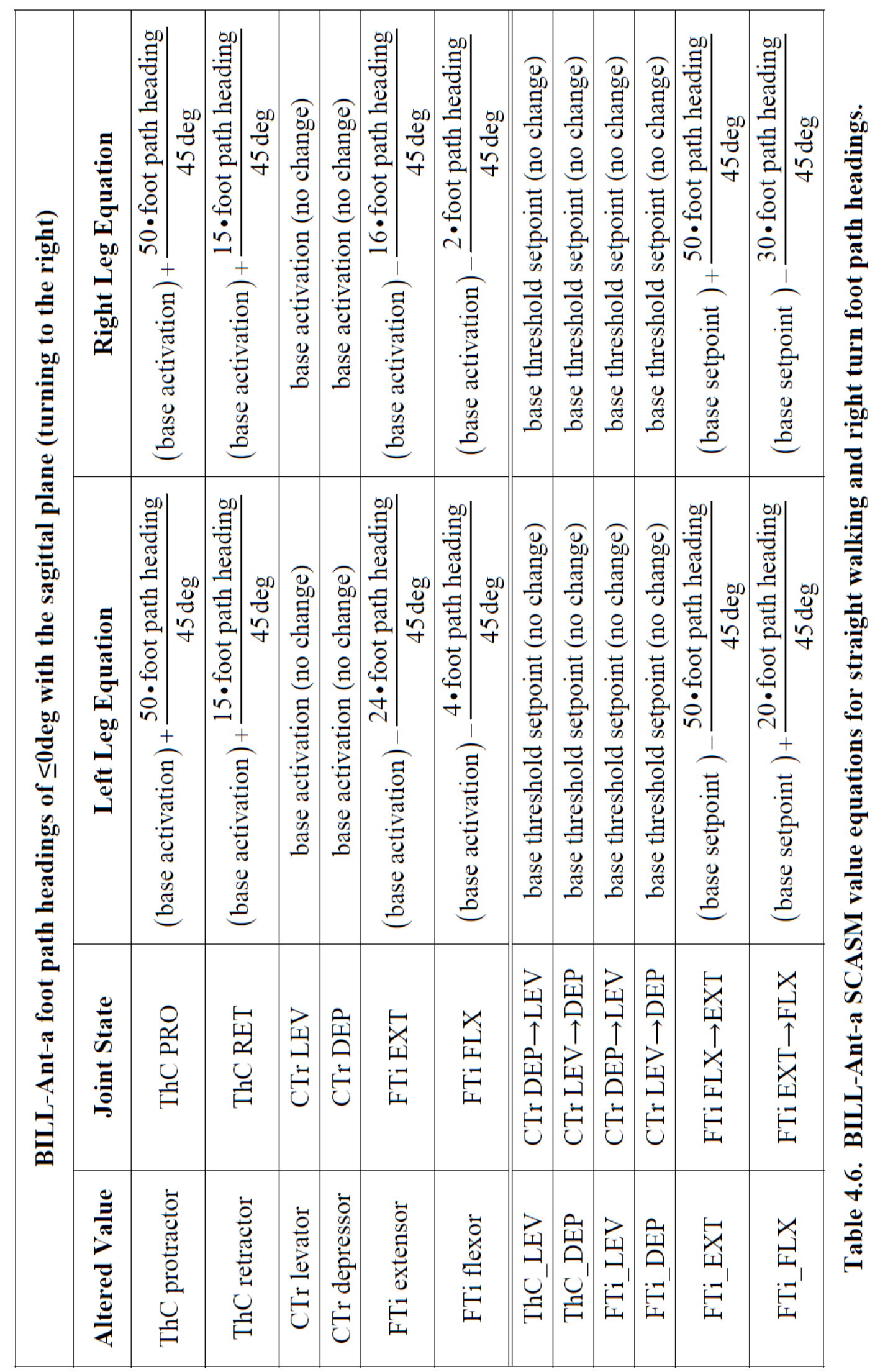




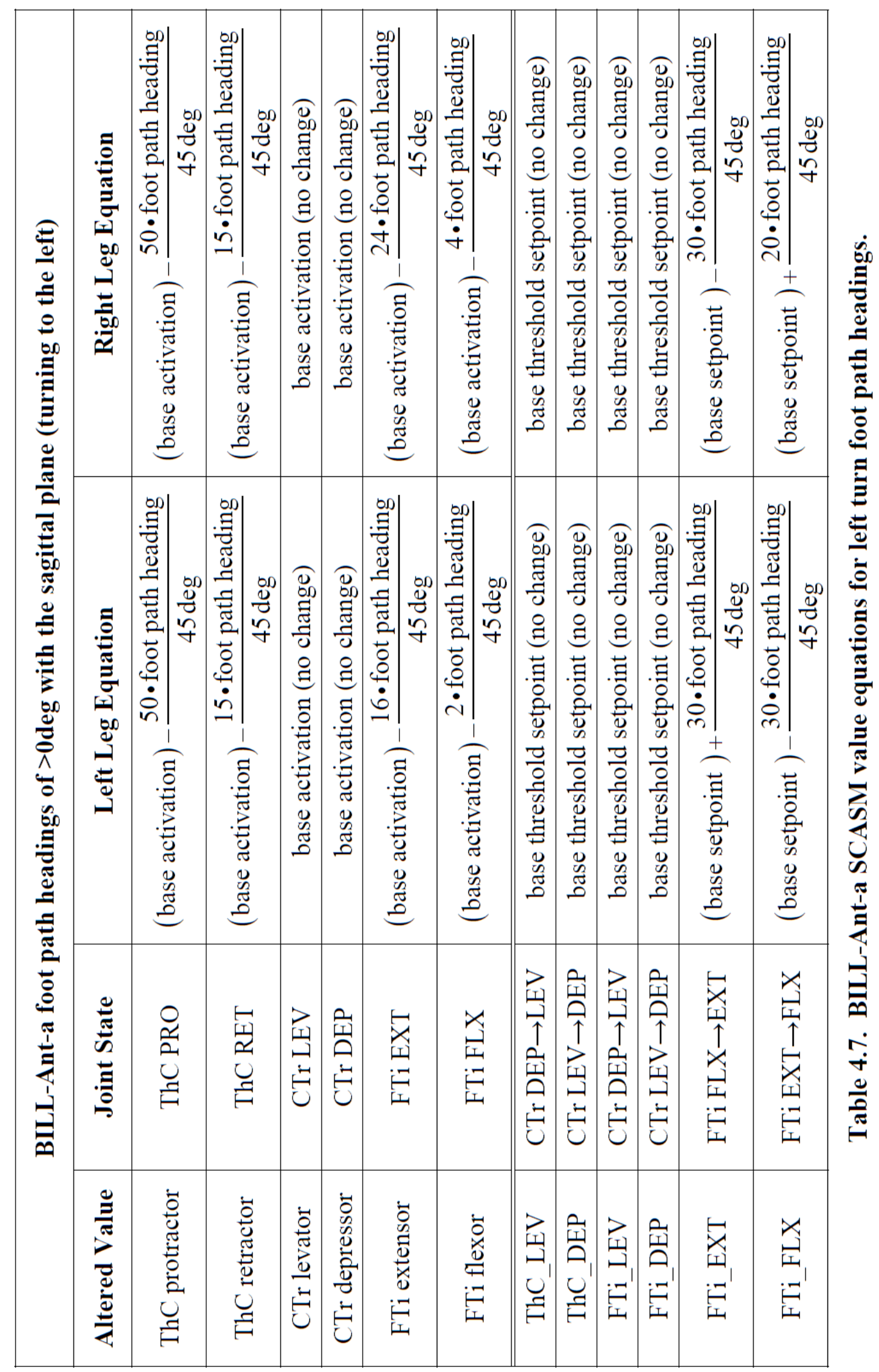


As a notable difference from the equations used with the simulator, there is no change in the TC_LEV and TC_DEP values for BILL-Ant-a. This is due to BILL-Ant-a using the same state transition value for each of those triggers, which corresponds to the apex of the swing phase (middle of the step length). This common value, both very far from the AEP and PEP extremes of the step path, is used because of the slow program speed of the BrainStem microcontrollers, relative to the joint motor speeds. When the original state transition thresholds from the simulator were used, the ThC joint of the robot would progress well beyond the indicated joint angles before the program was aware. In response to this behavior, the state transition threshold angles were placed farther from the step length extremes, and ended up becoming the same point at the center of the step.

When the neck and light-based goal seeking assembly was mounted on BILLAnt-a, some problems were observed. When the neck was actuated to track a goal, the phototransistors frequently moved within their mounting clips, thereby changing the orientations of the sensors and altering the perceived heading toward the goal. To rectify this, a new sensor assembly was fabricated (Fig. 4.22). This new assembly holds the two phototransistors in fixed positions relative to one another by mounting the sensors in a section of aluminum angle bracket. The bracket separates the two sensors by a distance of $3.0 \mathrm{in}(7.62 \mathrm{~cm})$.

As a side-effect of this new sensor assembly, the field-of-view was reduced from approximately $120 \mathrm{deg}$ ( $\pm 60 \mathrm{deg}$ from the sagittal plane) to only $45 \mathrm{deg}$. This led to 
BILL-Ant-a often not observing light-based goals, or losing sight of those goals due to the robot's movements while walking. To recover the lost field of view, a scanning behavior was implemented.

The scanning behavior, programmed into the "head" microcontroller, actuates the neck over its 90deg range-of-motion whenever a light source is not located. This scanning behavior, which is seen in insects (Bell 1990), increases the field of view to 135 degrees ( $\pm 67.5 \mathrm{deg}$ with the sagittal plane) and allows BILL-Ant-a to more repeatedly find and follow light-based goals.

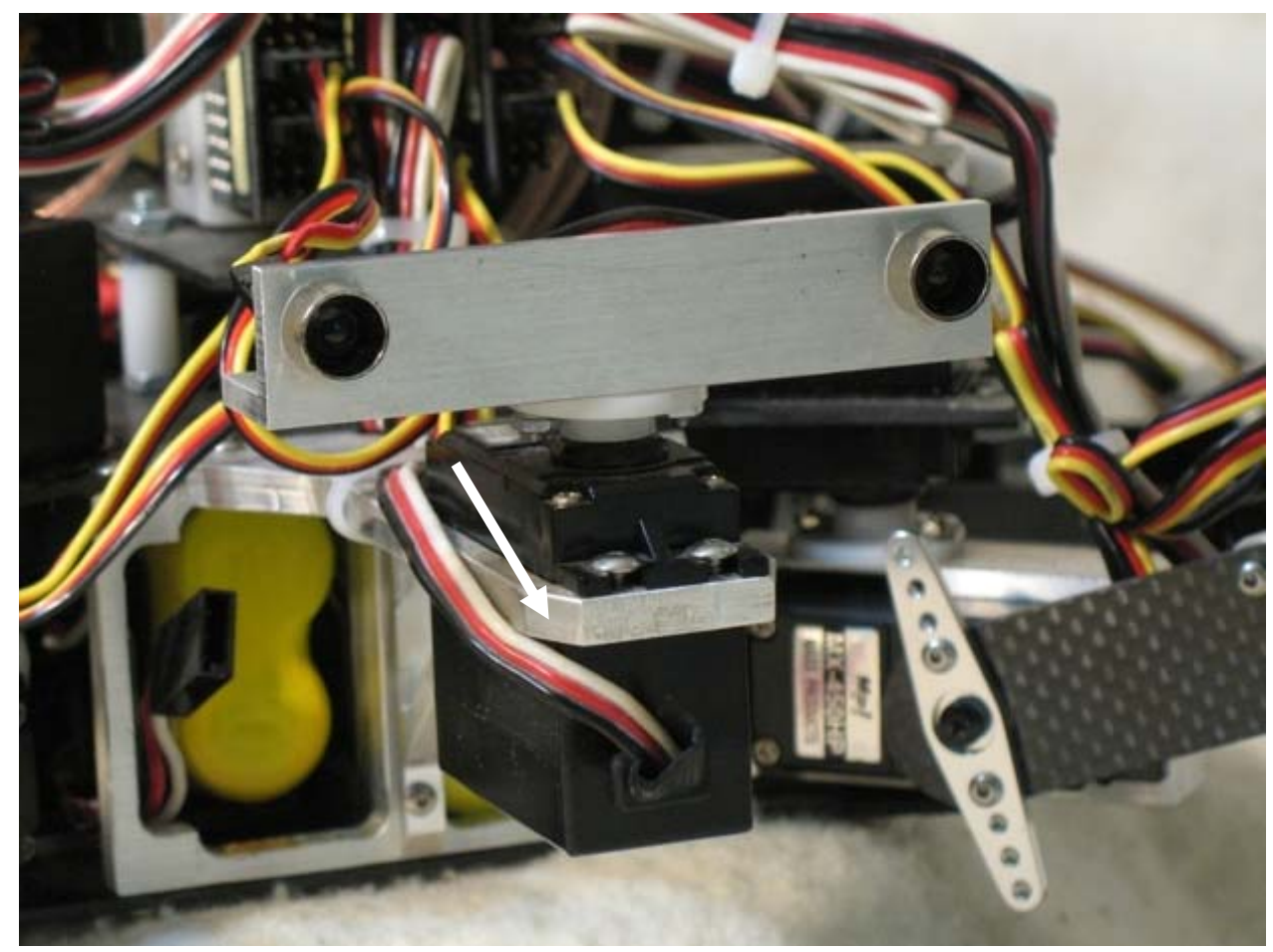

Figure 4.22. Updated phototransistor mounting bracket.

The neck servo motor has a scanning behavior that oscillates the neck over its $\mathbf{4 5 d e g}$ range-of-motion when a light-based goal is not observed. 


\title{
CHAPTER V
}

\author{
LEG COORDINATION
}

BIOLOGICALLY-INSPIRED LEGGED LOCOMOTION - LOW COMPUTATION POWER EMERGENT GAIT SYSTEM (BILL-LEGS)

\subsection{General}

For two decades, Holk Cruse's leg coordination method has been used as a control basis for generating gaits in legged robots. His method, based on observations of stick insect walking behaviors, has been successfully implemented in a number of robots such as Robot I (Espenschied et al. 1993) and Robot II (Espenschied et al. 1996), the TUM Walking Machine (Pfeiffer et al. 1994; Weidermann et al. 1994), Tarry II (Frik et al. 1999; Cruse et al. 2003), and BILL-Ant-p (Lewinger 2005; Lewinger, Branicky, and Quinn 2005). However, some engineers have had difficulties implementing the controller when trying to select robust mechanism influence weights that are immune to variations in starting pose and leg speed (Ferrell 1993). Additionally, the coordination method can be overwhelming or impossible for the low computation capable microcontrollers preferred for small, untethered mobile robots, such as the BrainStem microcontroller used with BILL-Ant-a.

The Biologically-Inspired Legged Locomotion-Low computation Emergent Gait System (BILL-LEGS) was developed as a solution to some of these issues. This method borrows heavily from Cruse's original design with some modifications 
that allow it to be implemented on small, autonomous legged robots using simple, low-end microcontrollers.

\subsection{Biological Inspiration}

Insects coordinate the motions of their legs into patterns within a continuum of metachronal gaits, as described by Wilson (Wilson 1966), to generate walking movements that propel them toward their goals. Decades of behavioral studies by Cruse and others into stick insect inter-leg coordination led to a set of mechanisms and a network commonly called "Cruse's method" that synthesize this continuum of insect gaits (Fig. 5.1) (Cruse 1990; Cruse et al. 2003; Dean 1991a). Currently, the underlying neurobiological pathways in insects that are responsible for gait generation remain undiscovered.

With Cruse's method, legs generate mechanism values that are weighted and applied as influences on neighboring legs. The legs receiving the combined influences of their neighbors can then have stance/swing and swing/stance transition points altered (adjusting the step length and foot placement) to form a coordinated series of gaits that vary with body speed.

The Cruse method for leg coordination has numerous mechanisms, each with a weighted value of influence on neighboring legs. Robot I (Espenschied et al. 1993), Robot II (Espenschied et al. 1996), and BILL-Ant-p (Lewinger 2005; Lewinger, Branicky, and Quinn 2005) each used a subset of these: Mechanisms 


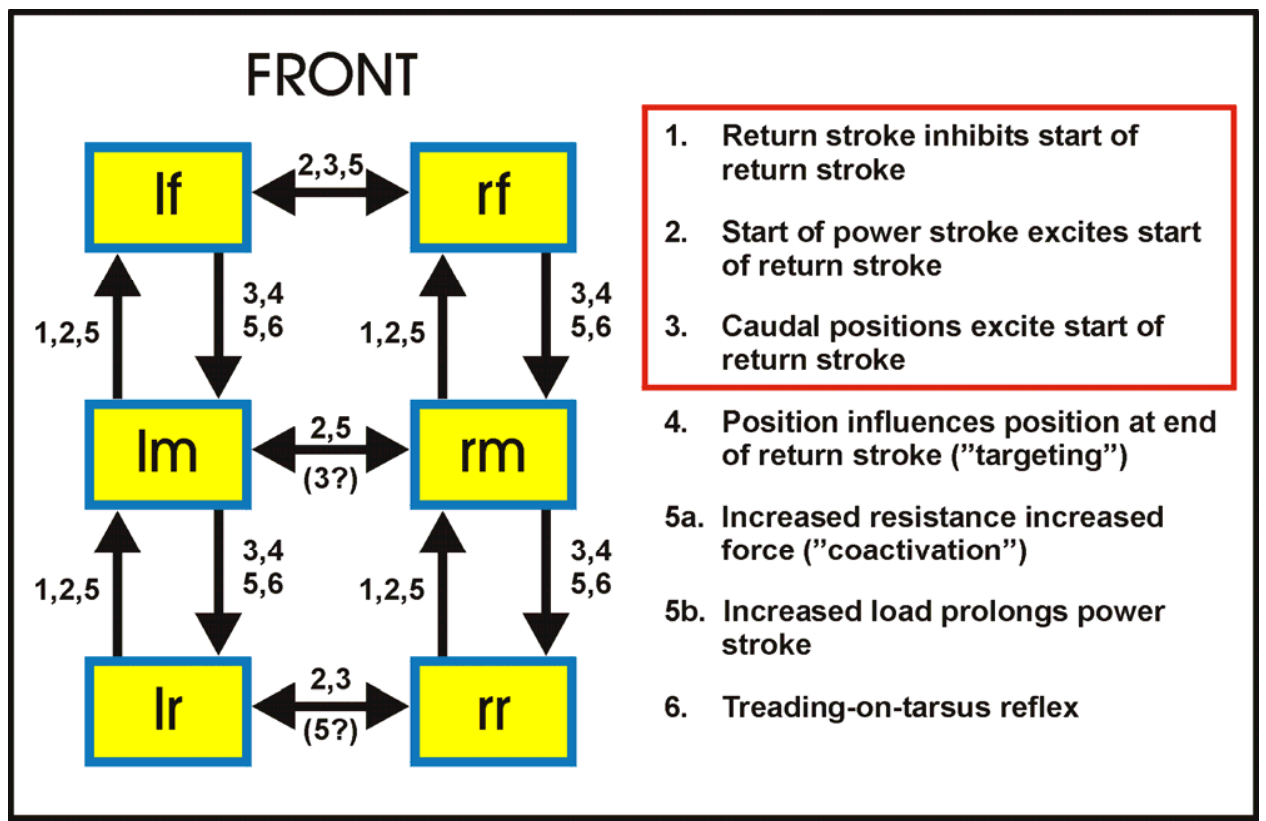

Figure 5.1. Mechanism connectivity network for Cruse's method of inter-leg coordination (Schmitz 1998).

The red box indicates a reduced set of essential mechanisms required for basic walking and were used in robots such as Robot I, Robot II, and BILL-

Ant-p.

1, 2, and 3. However, even with this subset, 8-12 unique mechanism weights needed to be calculated to allow a smooth continuum of statically stable gaits to form. For Robot I and Robot II, mechanism weights were manually chosen. For BILL-Ant-p, a genetic algorithm was used to find values.

One of the difficulties in selecting mechanism weights is that the chosen values affect which gaits emerge, if at all, and how stable the gaits are (Espenschied et al. 1993). While some of the mechanisms (i.e. Mechanism 2) are less influential than others in creating a smooth continuum of insect gaits that vary with body speed, others (i.e. Mechanism 3) are more crucial. There are also situations where 
certain values cause statically unstable postures that may not appear immediately, but emerge after many stepping cycles.

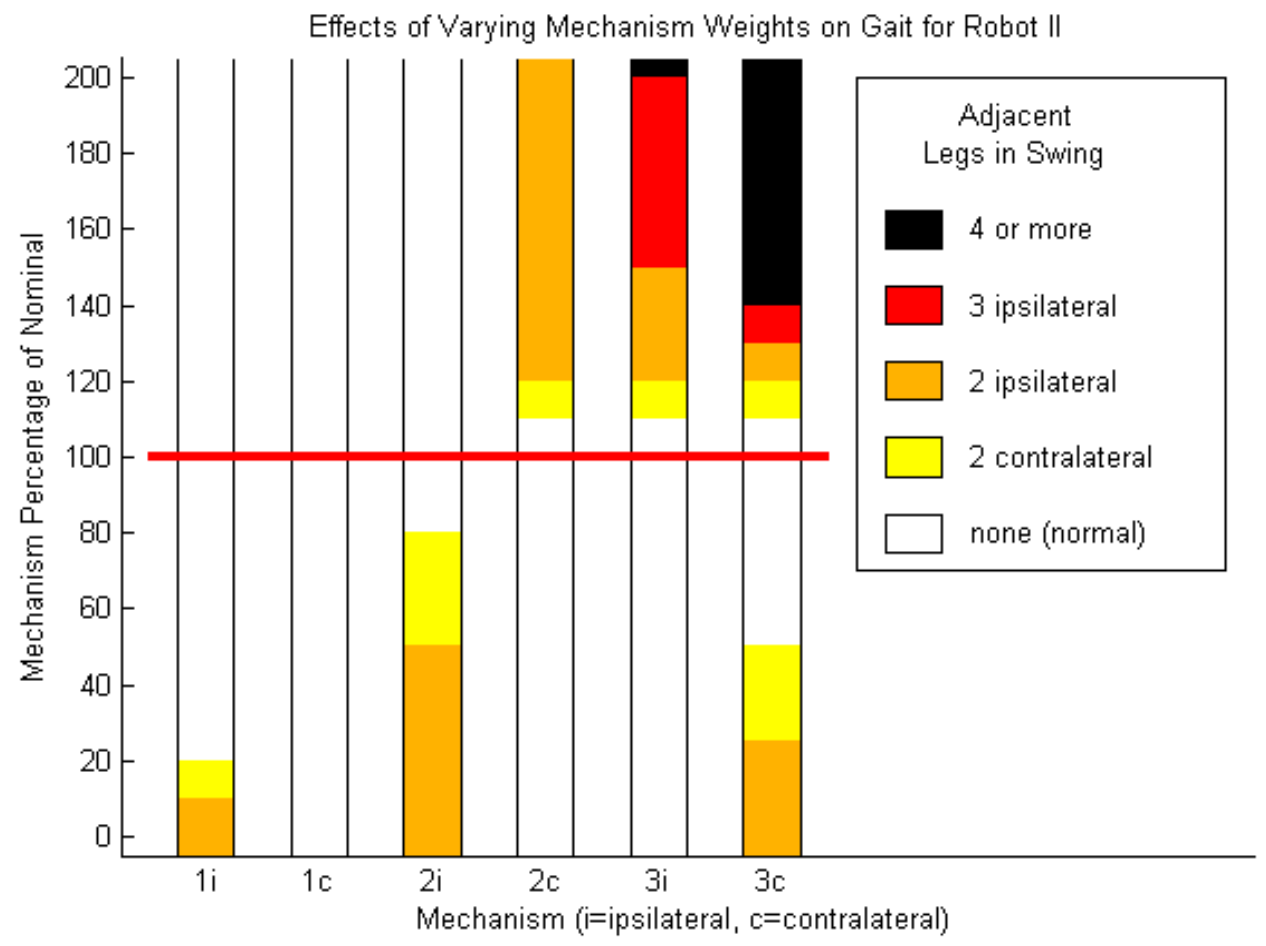

Figure 5.2. Immunity of leg coordination mechanism weights to variation in the generation of stable gaits for the Cruse method in Robot II.

The white bands indicate mechanism weight values that generate stable gaits.

The horizontal line at $\mathbf{1 0 0 \%}$ represents the nominal mechanism weight values.

Espenschied et al. 1996 presented data that showed the robustness of Cruse's method when selecting mechanism weights (Fig. 5.2). It was shown that Mechanism 2 was not very important to the formation of stable gaits, while Mechanism 1 was somewhat important, and Mechanism 3 was very important and the least robust to alteration. 
The controller for BILL-Ant-p also used Mechanisms 1, 2, and 3, but showed less robustness for these mechanisms to alteration (Fig. 5.3). It is believed that the axially-symmetric starting pose of the legs with respect to the sagittal plane may have generated fewer sets of mechanism weight candidates.

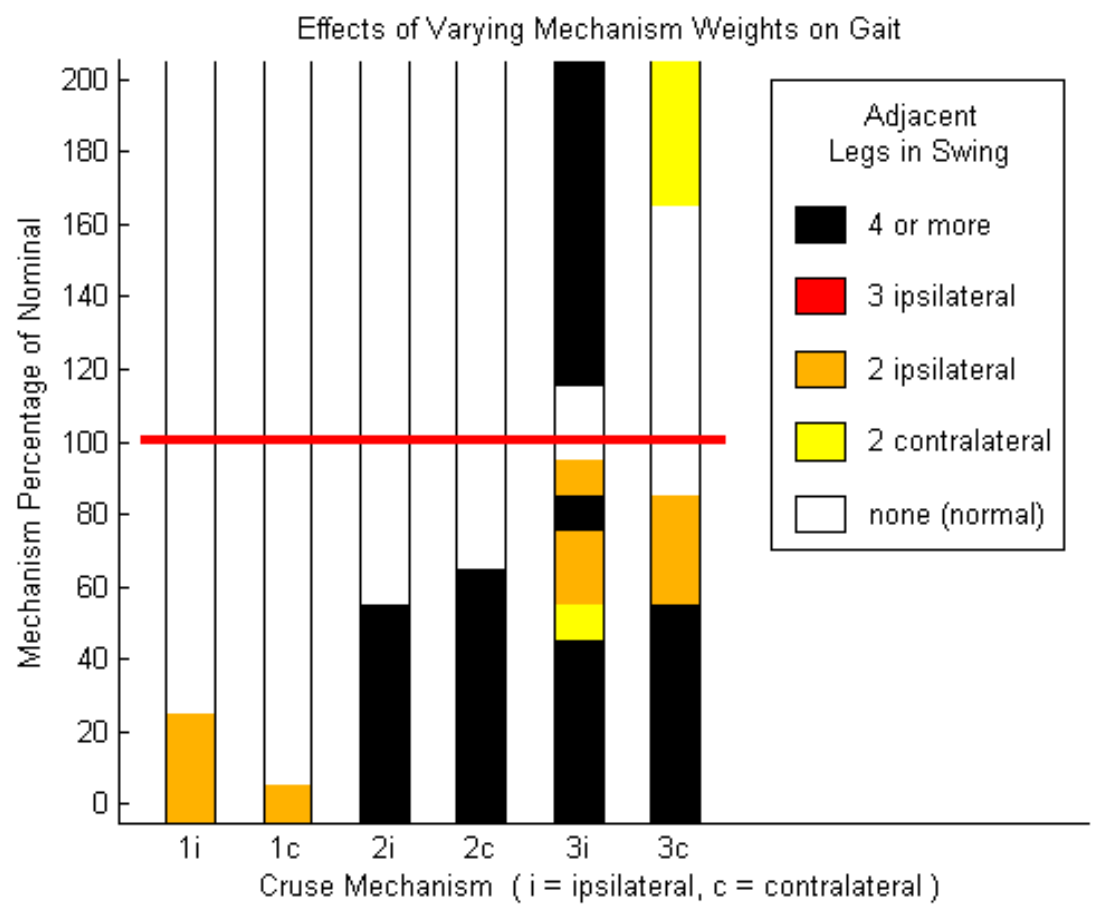

Figure 5.3. Immunity of leg coordination mechanism weights to variation in the generation of stable gaits for the Cruse method in BILL-Ant-p.

Note the smaller allowed variation of weights (smaller white spaces surrounding the horizontal line at $\mathbf{1 0 0 \%}$ ) to those used for Robot II (shown above in Fig. 5.2).

Further tests performed with BILL-Ant-p showed relatively small changes in starting pose and leg speed caused undesirable stepping patterns (Fig. 5.4). While the situation of two contralateral legs simultaneously in swing is seen in insects (e.g. in climbing (Watson and Ritzmann 1998)), this situation was undesirable for 

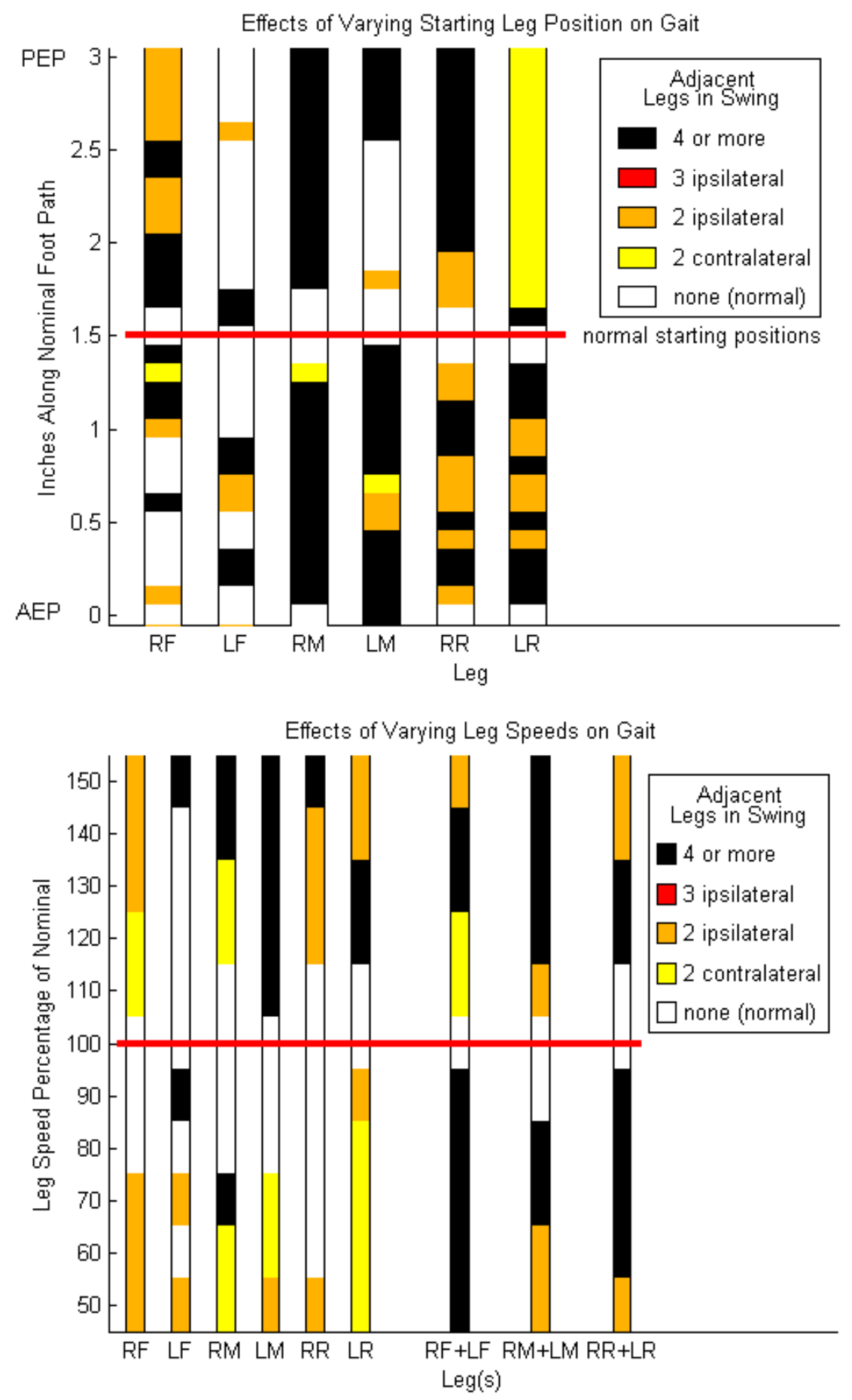

Figure 5.4. The effects of varying individual starting leg positions (top) and leg movement speeds of individual legs and leg pairs (bottom) on stable gait generation for the Cruse method in BILL-Ant-p.

The small white bands about the horizontal line at $100 \%$ indicate the lack of robustness to changes. Larger white bands would indicate more robustness. 
BILL-Ant-p. Since the center of mass in BILL-Ant-p is slightly forward of the middle Thoraco-Coxal (ThC) joints, the robot would fall forward if both front legs were in swing. Consequently, mechanism weights were chosen to not allow this situation for all gait patterns and body speeds.

\subsection{BILL-LEGS Implementation}

The BILL-LEGS method is very similar to Cruse's method for leg coordination in that it's a modular, distributed system in which neighboring legs influence one another by weighted mechanisms to form a speed-dependant continuum of gaits. A key difference is that BILL-LEGS uses only two mechanisms and a total of two system-wide mechanism setpoint values to coordinate inter-leg movements (Fig.

5.5). All legs use the same two setpoints, one for each mechanism; unique ipsilateral and contralateral values are not needed. Another major difference with the BILL-LEGS method is that the legs influence themselves in addition to their neighbors.

It is important to mention that BILL-LEGS does not perform all of the functions of Cruse's method. The omission of mechanisms limits its use for behaviors such as "follow-the-leader" where caudal legs step on positions previously occupied by rostral legs. These mechanisms were removed since they are more suited to extremely sparse environments, rather than the irregular terrains and somewhat sparse environments for which BILL-Ant-a was developed. 


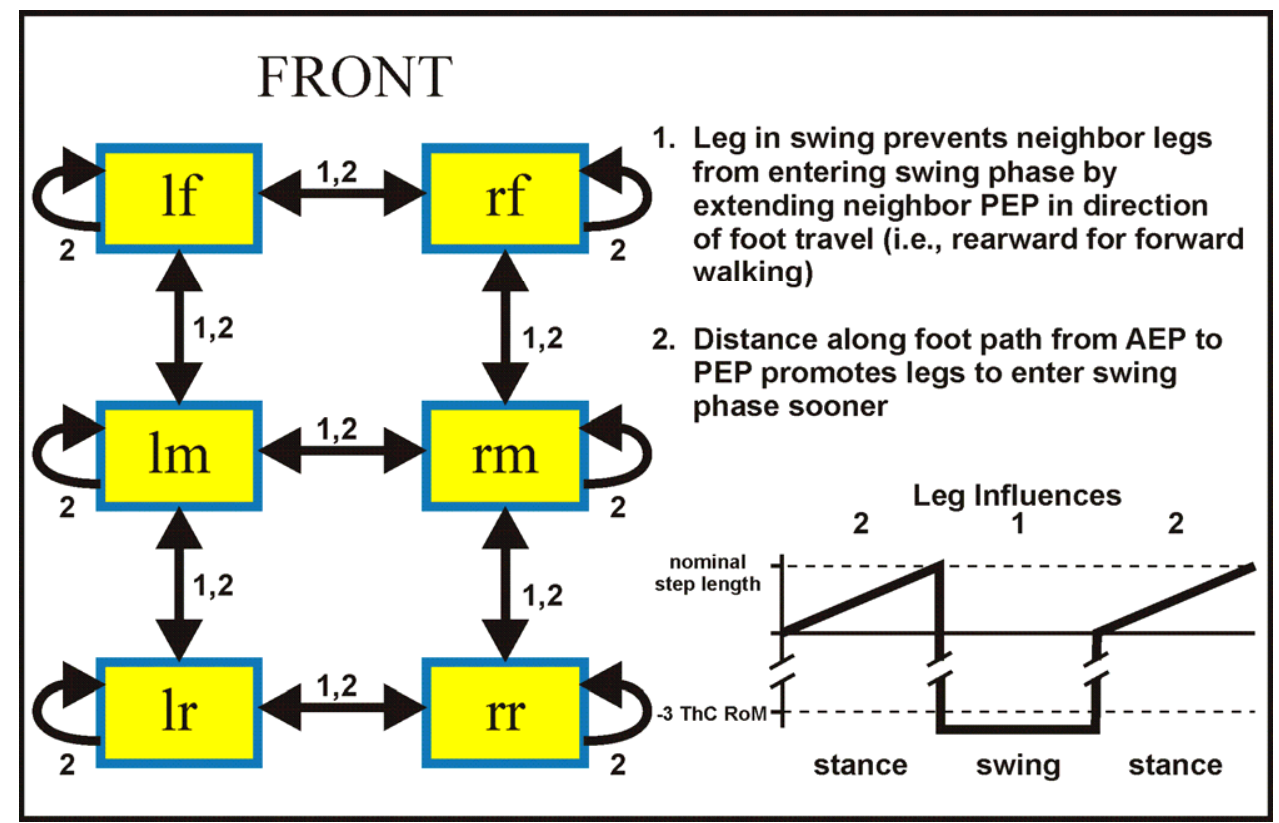

Figure 5.5. BILL-LEGS mechanisms and connectivity diagram.

The two mechanisms are similar to Cruse's mechanism 1 and mechanism 3 and influence each orthogonal neighbor bi-directionally. A key difference in BILL-LEGS is that the legs influence themselves as well as neighbors.

\subsubsection{Mechanisms and Leg Influences}

The first of two mechanisms is a fixed-level signal generated when a leg is in swing. The second is a ramp signal formed during stance that represents the percentage of the distance traveled from the intrinsic anterior extreme position (iAEP) (swing/stance transition point) to the intrinsic posterior extreme position (iPEP) (stance/swing transition point).

The iPEP position for BILL-Ant-a is partly determined by the TC_LEV state transition threshold setpoint, and the iAEP position is partly determined by the TC_DEP state transition threshold setpoint. These setpoints do not mark the exact iPEP and iAEP positions, however, since the iPEP and iAEP are defined as 
the points where the stance/swing phase and swing/stance phase transitions occur, respectively. These transitions are marked by the loss of (for the iPEP) or reacquisition (for the iAEP) of ground contact, so other factors such as step length and "muscle activation" values dictate the actual iPEP and iAEP points.

While in stance phase, each leg generates a positive signal between 0 and $100 \%$ as it moves from the iAEP to the iPEP (Mechanism 2). Positions beyond the iPEP generate signals larger than $100 \%$. When a leg is in swing, a large, fixed, negative value is used instead - -900 (Mechanism 1) (Eq. 5.1). This value is chosen to be more than three times larger than any possible Mechanism 2 value that a leg in stance could generate, larger than the entire range-of-motion in fact, regardless of its iAEP and iPEP positions. Once all the mechanism influences are generated, they are shared with orthogonally neighboring legs (Fig. 5.5).

$$
\begin{aligned}
& \text { stance influence }=(\text { foot position }-i A E P) / \text { foot path length } \bullet 100 \\
& \text { swing influence }=-900
\end{aligned}
$$

Typically, a step length is about half of the range-of-motion. Multiplying this by two to cover the entire RoM, and then by three to account for all neighboring legs results in $600(100 \%$ step length $* 2$ for RoM $* 3$ for neighboring legs $=600)$. It is possible, though highly unlikely, for a leg and two neighbors to have just arrived at the end of their respective RoM positions and having the nominal step length of RoM / 2. With the one neighbor leg in swing phase, this would generate a total leg influence value of $0\left(200+\sum(200+200-600)=0\right)$. To alleviate this possibility, the swing phase influence is set $50 \%$ greater to -900 . 
Net influence values are calculated for each leg in stance during each control cycle (Eq. 5.2). The influence calculation begins with the positive influence of the current leg on itself (a difference from Cruse's method), and then adds the influences of orthogonal neighbor legs.

$$
\text { net influence }=\text { leg self influence }+\sum \text { neighbor influences }
$$

By using a swing phase influence weight of -900 , the net influence is negative when at least one neighboring leg is in swing. When the net influence is negative the PEP for the current leg is set beyond the extreme RoM position in the direction of leg travel (i.e., beyond the most rearward position physically reachable when the robot is walking forward), which keeps the leg in stance longer. This is the "step late" condition (Fig. 5.6). Since the leg cannot reach this position, it never meets the criteria to enter swing. If a neighboring leg in swing takes too long to begin its stance phase, the current leg will drag at its physical limit, although this rarely happens since the swing speed is at least twice the stance speed and the nominal step length is about half of the RoM.

The "step early" condition is calculated by Eq. 5.3. The neighbor leg that is farthest along its foot path during stance is found by using the max function, since leg influences generate increasing ramp signals while in stance. If a neighbor is at least half way through its stance phase and the current leg is farther along its own foot path, then the current leg will have its PEP set to the current position of the foot, which then causes the leg to step early, by beginning its swing phase immediately. This "step early" behavior reduces or eliminates the amount of time 


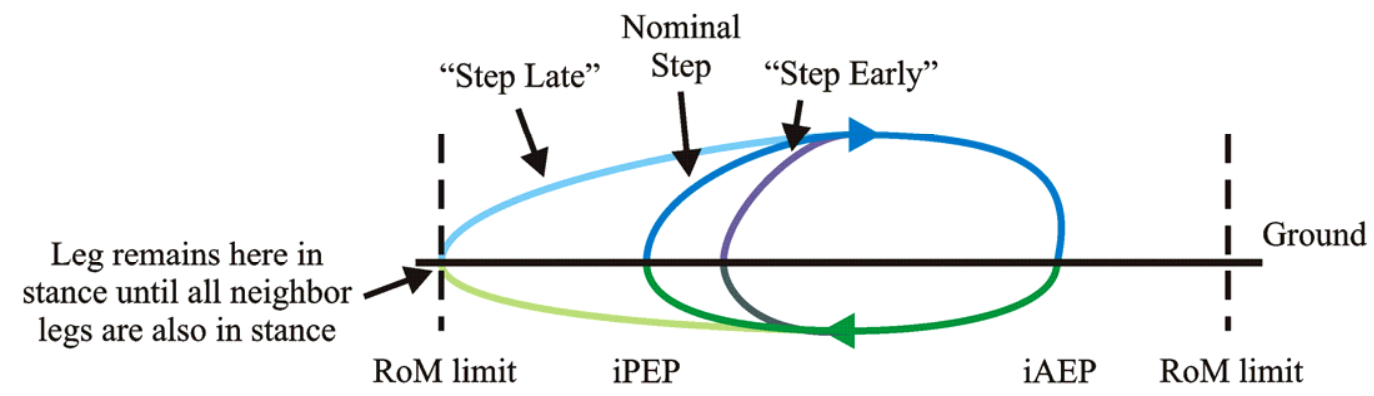

Figure 5.6. Nominal, "Step Late", and "Step Early" foot path diagrams.

Normally, the foot path follows the Nominal Step line. When at least one neighbor leg is in swing phase as the current leg passes its iPEP, the stance phase is extended up to the leg RoM. The swing phase will then begin once all neighbor legs are in stance phase. If a neighbor leg is at least half way through its nominal step and the current leg leads that neighbor, then the current leg will step early in order to reduce (or eliminate) the amount of time that a neighbor remains in stance phase beyond its iPEP.

that a neighbor leg will need to remain in stance phase beyond its iPEP due to Mechanism 1. Since the swing phase speed of the foot is at least twice that of the foot in stance phase, the foot that steps early will be able to complete its swing phase before the neighbor leg reaches its iPEP (given that the nearest neighbor leg has just reached the halfway point in its foot path). When the neighbor is farther along its own foot path, then it will need to wait in stance beyond its iPEP while the current leg completes its swing.

When neither of the conditions in Eq. 5.2 nor Eq. 5.3 is met, then the current leg will enter its swing phase at the normal point, its iPEP.

$$
\begin{aligned}
& \text { IF } \max (\text { neighbor influences }) \geq 50 \% \text { step path length } \\
& \text { AND } \\
& \text { IF leg self influence }>\max (\text { neighbor influences }) \\
& \quad \text { THEN } \\
& \text { set PEP to current leg position }
\end{aligned}
$$




\subsubsection{Simulation Setup}

A simple simulation environment was created using MATLAB R2006b (The MathWorks, Inc., Natick, MA, USA) that modeled a 2-DoF/leg hexapod. Legs were capable of protracting and retracting with a Thoraco-Coxal (ThC) joint, and had a second DoF that allowed them to be in either swing or stance. $9 \mathrm{~cm}-$ long legs (representing the coxa, trochanter, and femur) were mounted perpendicularly to a central, $20 \mathrm{~cm}$-long thorax and had ranges-of-motion of \pm 45 degrees. The dimensions used were measured from the physical BILL-Ant-a robot while in a stance phase posture. The tibia and foot segment length was not used as that segment is typically oriented vertically and adds no length to the leg when viewed from above, hence the seemingly short $9 \mathrm{~cm}-$ long leg length. The $20 \mathrm{~cm}-$ long thorax size was measured from the BILL-Ant-a body section. Swing speeds were set to $333 \mathrm{deg} / \mathrm{sec}$, which is the maximum speed of the servo motors used in the BILL-Ant-a ThC joints.

The simulator used all integer values (similar to the low computation capable BrainStem microcontroller) and the 'percentage along the foot path' measurement (BILL-LEGS Mechanism 2) was based on the ThC joint angle with a nominal stance length of 50 degrees ( \pm 25 degrees perpendicular to the thorax).

Because the simulator was developed as a single piece of software, each leg's calculations were performed sequentially. This differs from the actual robot which has distributed controllers that perform the calculations asynchronously in parallel. As the performance characteristics of the six BrainStem microcontrollers 
are similar, this difference in implementation was not seen as being wholly unrepresentative of real-world scenarios.

The number of calculation cycles (duration of the simulation), starting and ending stance speeds (as a percentage of swing speed - used to dictate the simulated robot walking speed), and over how many calculation cycles a ramp-up or rampdown of stance speed was to occur (if a speed change was specified for a given test) could be set at run time. The outputs of the simulator were several plots and two animations.

This environment also allowed independent setting of the iAEP and iPEP values, starting ThC angles (corresponding to starting leg positions and therefore a starting pose for the robot), and joint actuator speeds for each leg. By entering different iAEP and iPEP values, the step lengths could be changed to create turning movements using a form of skid steering, where legs on the inside of the turn moved slower and over shorter step lengths. While this is not how insects perform turning actions (Dürr and Ebeling 2005), it was all that could be accomplished with 2-DoF legs. It was demonstrated, however, that while the simulated foot trajectories differed from actual insect foot paths during turning (the simulator restricted foot path movements to arcs that are parallel to the sagittal plane, while insects alter their foot path heading angle when turning), the stance duration was longer for outside legs than inside legs, similar to what Dürr and Ebeling 2005 showed. 


\subsubsection{Simulation Results}

Several experiments were performed to test various aspects of the system. Important key points during the experiments were to thoroughly test if neighboring legs could simultaneously be in swing (testing static stability) and how quickly insect gaits formed with a variety of starting poses, joint actuator speeds, and mechanism setpoints (testing the formation of a continuum of speeddependent gaits).

To test if neighboring legs could be in swing at the same time, the simulator was programmed to repeatedly run through trials of 5,000 calculation cycles (corresponding to approximately 28 steps for each leg). Stance speeds were ramped up from 0 to $50 \%$ of swing speed, which is constant, within the first 2,000 calculation cycles and held for the remaining cycles. Random ThC starting angles (within the entire $\pm 45 \mathrm{deg}$ range-of-motion) and random joint actuator speeds were also used during the trials. 10,000 trials were performed. At no time in any of the trials were neighboring legs simultaneously in their swing phase, showing that the controller produced statically stable gaits.

The next major aspect of testing was to see how gaits were generated as stance speeds were increased. For these trials, stance speed started at 0 and was increased to $50 \%$ of swing speed over the first 2,000 of the 5,000 calculation cycles during straight-line walking. The maximum stance speed of 50\% swing speed was chosen as the point where the legs should be in an alternating tripod 
gait, according to work done by Kindermann and others (Kindermann et al. 1998).

The first series of gait tests were performed with identical joint actuator speeds for each leg. Three sets of starting ThC angles were used. The first set intentionally promoted a wave gait, the second began with all legs perpendicular to the thorax, and the third used randomly selected angles.

As seen in Fig. 5.7, the first test shows a continuum of gaits from wave to tetrapod to tripod. The second test, where the starting angles were perpendicular to the thorax, had all legs traveling rearward from the same points in their respective step lengths and at the same rates. Since the leg influences and swing/stance phase were computed for each leg beginning with the left-rear (due to the way that the simulator was programmed to perform calculations sequentially beginning with the left-rear leg), a tripod was formed with the leftrear, right-middle, and left-front legs while the other legs had their PEPs extended and remained in stance. As soon as the first tripod of legs completed their swing phases, the remaining legs entered swing. Because of the starting positions of the legs, the wave and tetrapod gaits were not seen for this test. 


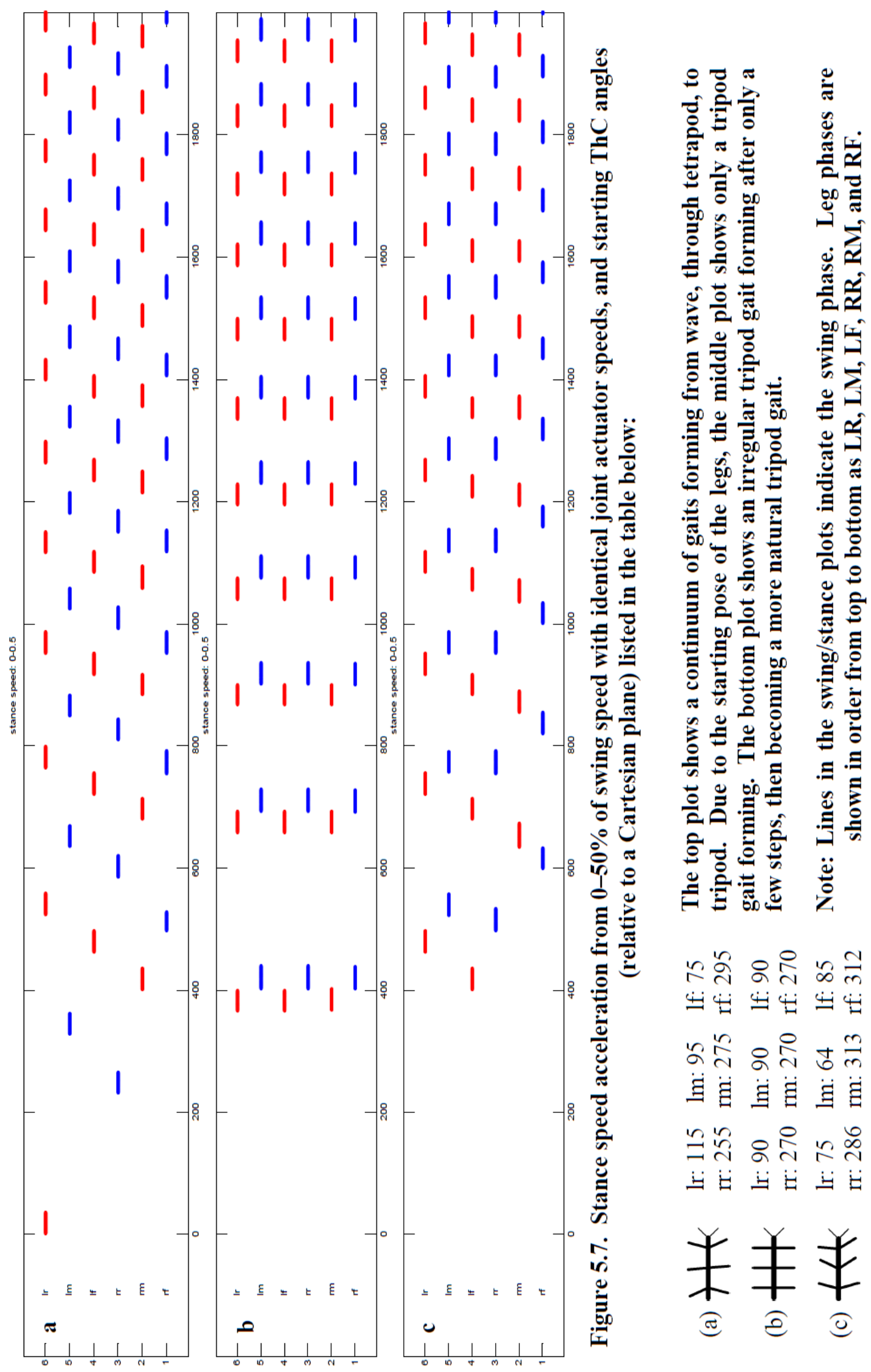


The bottom plot of Fig. 5.7 shows a similar test with random ThC starting angles. Note how the seemingly disjointed steps in the beginning become an orderly tripod gait as stance speed increases. A total of 10,000 trials were performed using random ThC starting angles; all trials produced statically stable results.

The next series of tests on gait formation used the same starting ThC joint angles as the first test of the previous gait formation trials (angles that promoted an initial wave gait), but allowed the joint actuator speed (for both stance and swing) to vary from $\pm 5 \%$ to $\pm 25 \%$ of nominal. Joint actuator speeds were randomized at the beginning of each of 10,000 trials per speed variance and maintained for the duration of each test. All trials used straight-line walking with a ramp up of baseline stance speed (stance speed before randomization) from 0 to $50 \%$ of swing speed. Result samples are shown in Fig. 5.8.

A continuum of gaits was seen and the standard alternating tripod gait was eventually formed in each trial with up to $\pm 15 \%$ variation in actuator speed. The final gaits for the $\pm 20 \%$ and $\pm 25 \%$ trials resembled alternating tripods, but were not as well-formed as in previous tests.

For the trial shown in Fig 5.8b, one tripod was manually chosen to have identical joint actuator speeds, while the other tripod had two of the legs set to a fast speed and one of the legs set to an intentionally conflicting slow speed. This was done to see how extreme disparities in joint actuator speeds would affect the formation of gait patterns while also maintaining static stability. 


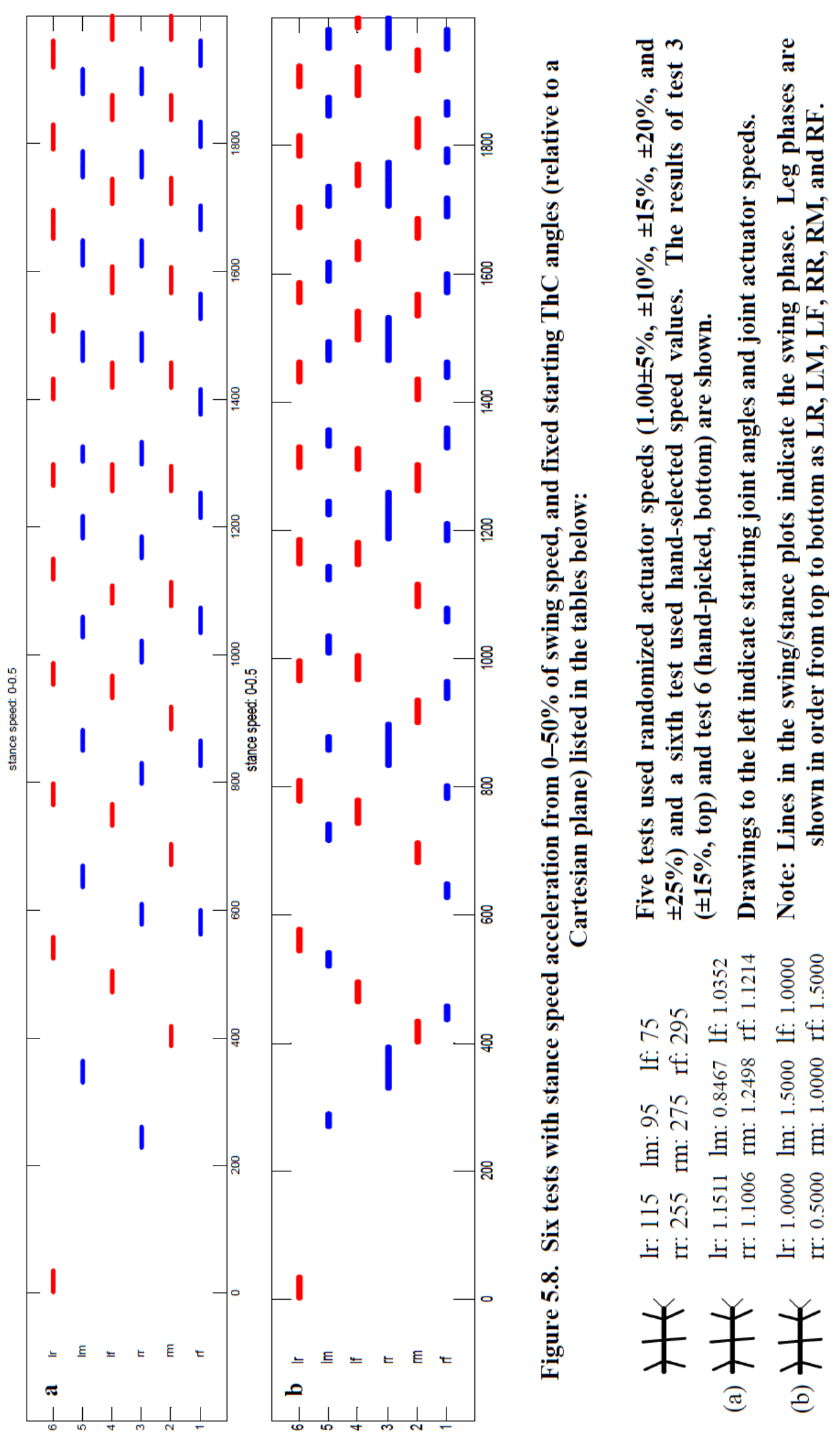


The slow right-rear joint actuator in Fig. $5.8 \mathrm{~b}$ can be identified by the long stance and swing phases. The slow speed led to missing every other swing phase cycle with the other legs in its tripod, the left-middle and right-front legs. The other tripod (left-rear, right-middle, and left-front legs), with similarly-matched actuator speeds, maintained their synchronized stance and swing phases despite the lesscoordinated actions of the other legs.

Additional tests were performed with the stance speeds increasing in stages: 0 $10 \%, 10-20 \%, 20-30 \%, 30-40 \%$, and $40-50 \%$ of the swing speed, with each stage lasting 10,000 cycles $(8,000$ cycles to accelerate and 2,000 cycles spent maintaining the maximum speed for the stage). These slow acceleration tests were conducted to ensure that the gaits had more than sufficient time to stabilize before increasing the speed. These tests showed similar results to previous tests, indicating that the continuum of gaits produced during the earlier trials were indeed stable, and not transitory.

It should be noted that not all gaits were observed with variations on starting poses and leg movement speeds as the baseline stance speed increased from $0-$ $50 \%$ of swing speed. For ideal conditions, the wave, tetrapod, and tripod gaits were seen. In different scenarios, however, only a subset of gaits emerged, such as tetrapod and tripod, or only tripod. These results are not surprising in light of similar findings by Cruse et al. 2003:

Actually very different step patterns can be observed e.g. after a brief disturbance of the movement of a single leg or when animals start walking from different leg configurations (Graham 1972; 
Dean and Wendler 1984). Insect gaits may therefore better be described by the term "free gaits" (McGhee and Iswandhi 1979). The usually observed tripod or tetrapod patterns represent limit cycle solutions that are only apparent in undisturbed situations (Cymbalyuk et al. 1998).

It is important to clarify that while the observed gait progressions varied with starting and operating conditions, which determined how many of the gait series were observed, there were no statically unstable gaits generated.
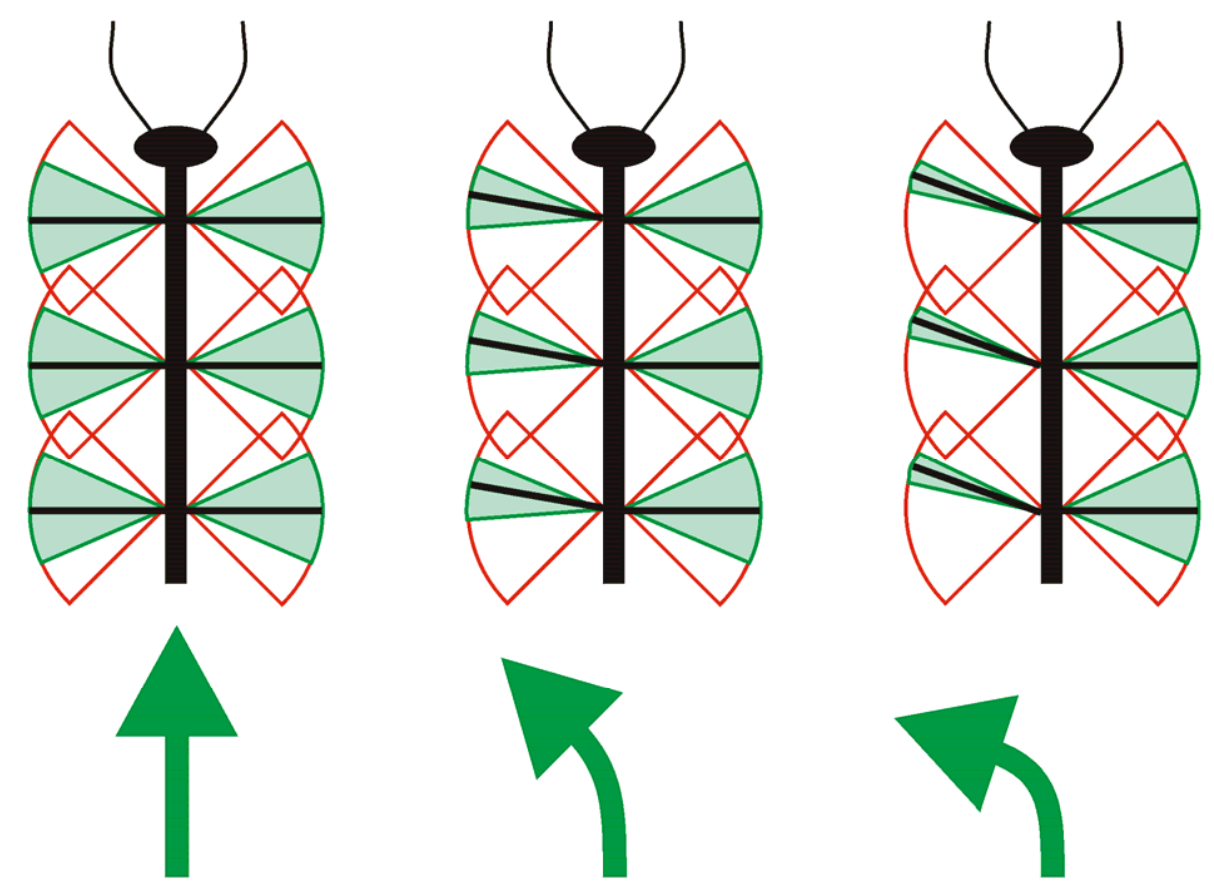

Figure 5.9. Variations in iPEP positions induce turning.

By moving the IPEP positions closer to the iAEP positions the step length is reduced. Performing this adjustment for all legs on one side of the robot, while proportionately reducing the leg speed, induces a "skid steering" type of turning.

After performing the gait formation experiments a series of turning trials was performed. These trials tested gait stability while adjusting iPEP values of the left 
legs to be closer to the iAEP values, thus shortening the foot path (Fig. 5.9). Since leg speed is a function of stance length in the simulation, legs with short foot paths (inside legs) move more slowly than those with longer foot paths (outside legs).

Three tests were performed with stance speeds fixed at 50\% of swing speed (no ramp-up period) and no randomized speed adjustments (Fig. 5.10). Starting ThC angles were set perpendicular to the thorax for each leg during all of these tests.
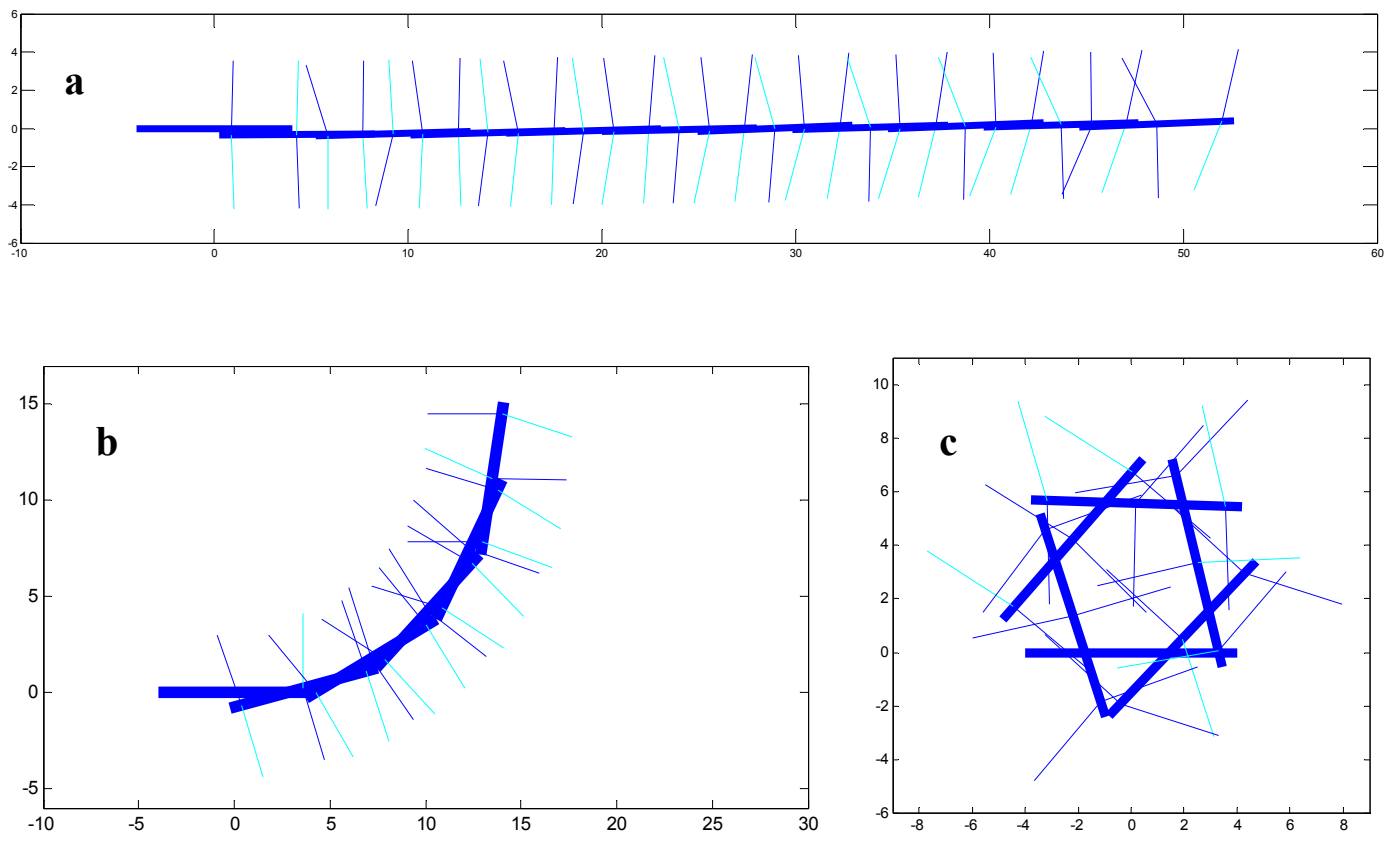

Figure 5.10. Composite images of the body and legs with fixed 50 degree foot paths for right legs, and decreasing foot path lengths for left legs.

(a) left: 50 degrees right: 50 degrees

(b) left: 30 degrees right: 50 degrees

(c) left: 5 degrees right: 50 degrees 
The straight-line walking test (Fig. 5.10a) used 50 degree step lengths for all legs (i.e. the step length was based on the $\mathrm{ThC}$ joint moving through a 50deg arc). The left arc test (Fig. 5.10b) maintained the step length for the right-side legs, but shortened the left-side legs to have step lengths of only 30 degrees. With this disparity in step lengths, a 90 degree turn was formed that had a turning radius of about 14.0in $(35.6 \mathrm{~cm})$. Reducing the left-side step lengths to 5 degrees created a tight left turn (Fig. 5.10c) with a radius of approximately $3.5 \mathrm{in}(9.0 \mathrm{~cm})$.

Fig. 5.11 shows a series of swing/stance plots for each of the three turning trials of Fig. 5.10. The left-side legs have progressively smaller swing phases for each of the trials due to the shortening of the foot paths for each successive trial. At the same time, longer stance phases are seen for each successive trial since the left-side legs also have proportionately slower joint speeds while in stance phase.

One final series of tests was performed to further determine the robustness of the BILL-LEGS method to changes in mechanism setpoint values, starting pose, and joint actuator speed. As shown in Fig. 5.12, BILL-LEGS is extremely robust to variations on system parameters, starting leg positions, and joint actuator speeds. While the types of gaits that were generated varied as the setpoints changed (i.e. not all of the wave, tetrapod, and tripod gaits were seen for each set of setpoints; at times only subsets of the gait patterns were observed), all of the gaits that were formed were statically stable.

It was observed, however, that the Mechanism 1 setpoint needed to be $\leq 0$ to be effective (Fig. 5.12, top). When the setpoint was $>0$, the legs moved to the iPEPs 
and remained there without initiating a swing phase. This is as expected, since the Mechanism 1 setpoint being $\leq 0$ corresponds to at least one neighboring leg being in its swing phase, so the net influence becomes negative (Eq. 5.2). Since the Mechanism 1 influence of -900 is very large, minor adjustments to the setpoint being $>0$ are tolerated, which is seen in small amount of white above the red line in the left-hand column of Fig. 5.12, top.

\subsubsection{BILL-Ant-a Implementation}

For the implementation of the BILL-LEGS method on BILL-Ant-a, no changes were made to the function calculations (Eq. 5.1, Eq. 5.2, and Eq. 5.3).

The calculation of leg influence values (Eq. 5.1) is performed in the sensex.tea programs. The results are placed into the Scratch Pad shared memory for use with the statex. tea programs.

The "step late" and "step early" calculations of Eq. 5.2 and Eq. 5.3, respectively, are conducted in the statex. tea programs and directly alter the direction of the $\mathrm{CTr}$ joint. For example, if the joint is in levation due to the $\mathrm{ThC}$ joint retracting beyond the TC_LEV position, but a neighbor leg is currently in its swing phase, the result of Eq. 5.2 will alter the $\mathrm{CTr}$ joint state to become depression, thereby prolonging the stance phase. Similarly, if the conditions specified in Eq. 5.3 are met, the CTr joint state, which would normally be in depression during stance phase, is adjusted to the levation direction, so that the 


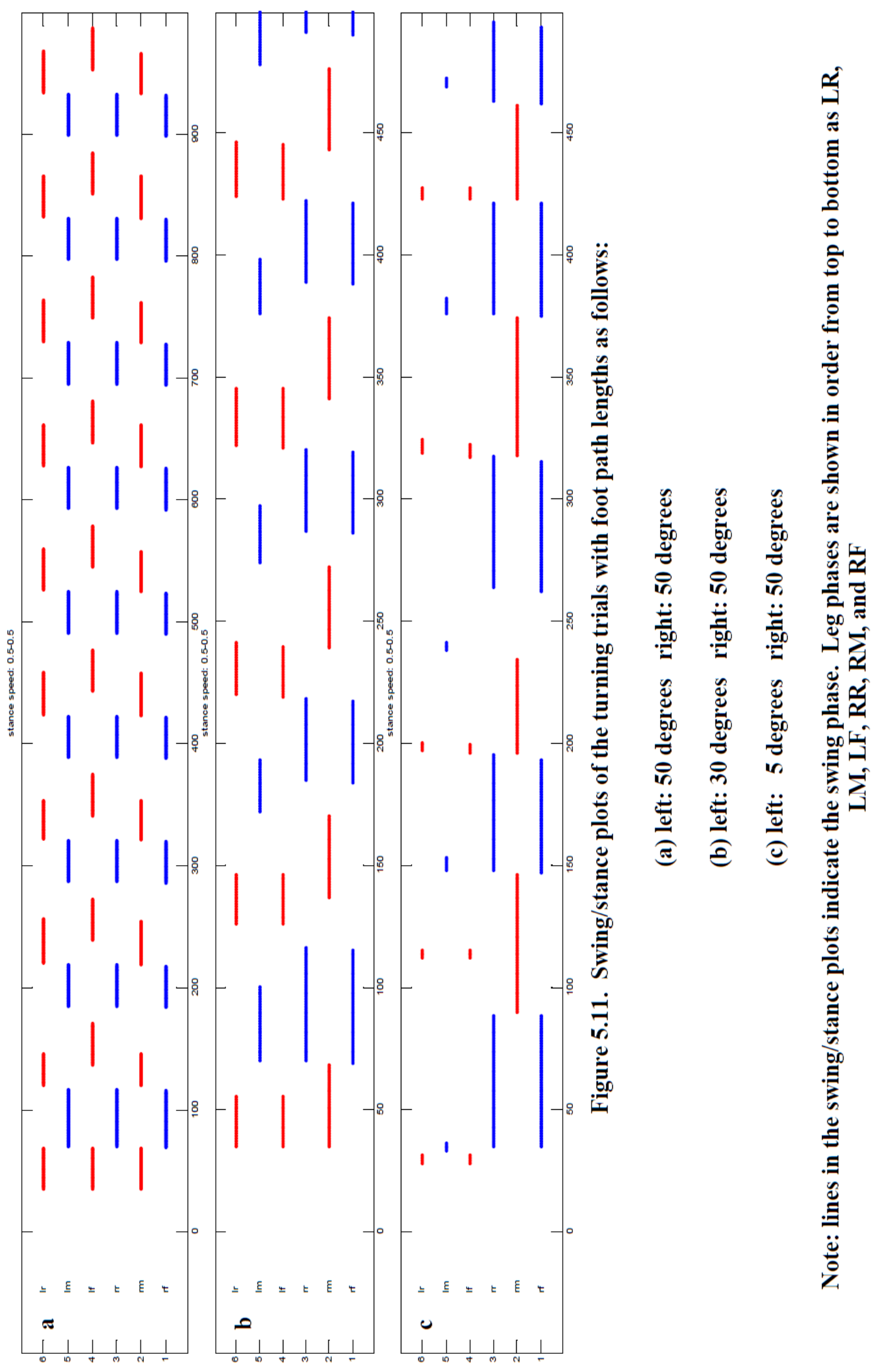


$\mathbf{a}$

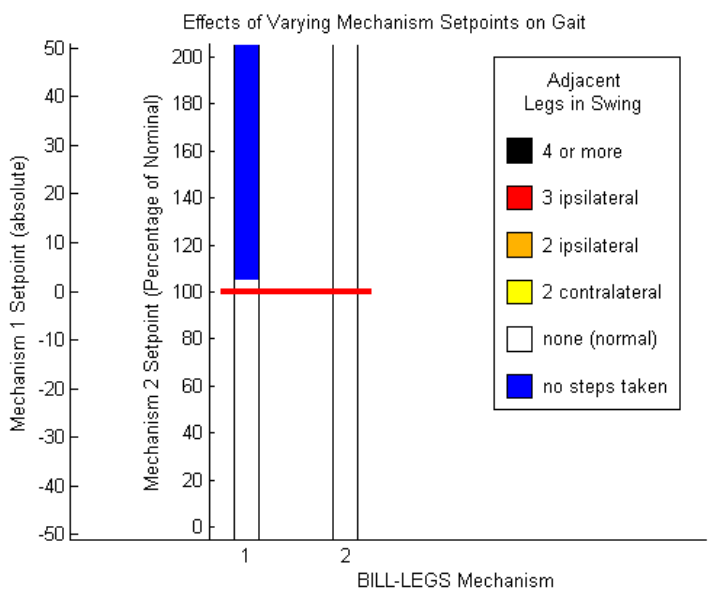

b

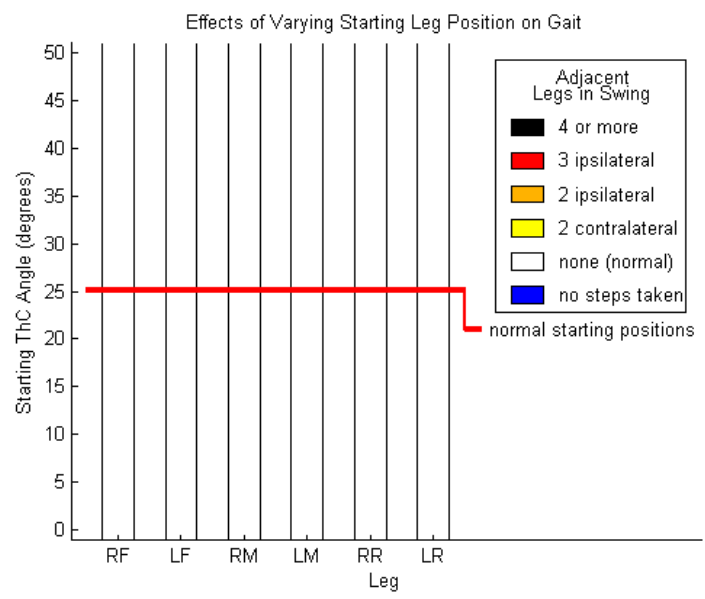

c

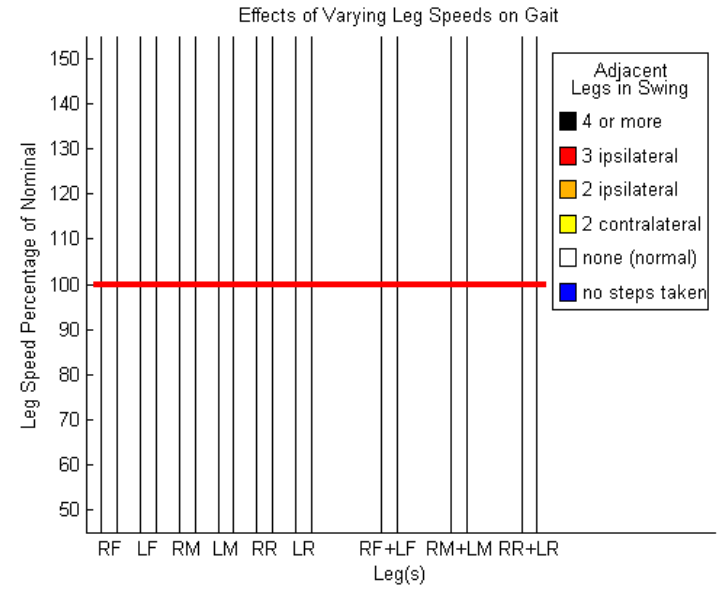

Figure 5.12. Robustness of BILL-LEGS to variations in a) mechanism setpoints, b) starting leg positions, and c) leg movement speeds.

Varying any parameter results in statically stable gaits, with the exception of the mechanism 1 threshold, which is limited to values $\leq 0$. Threshold values $>0$ prevent leg transitions from stance to swing phase, and the legs remain standing at the PEP locations. 
swing phase for that leg begins sooner. When neither of the conditions of Eq. 5.2 or Eq. 5.3 is met, the normal SCASM-defined stepping behavior is performed according to nominal state transition threshold values. 


\section{CHAPTER VI}

Project DeVElopment AND LeSSONS LEARNED

\subsection{General}

Many incremental steps were taken during the development of the BILL-Ant-a project. Setting intermediate goals within the framework of the entire project allowed progress to be better evaluated and managed. This became more important when component integration was being performed and when new methods and practices were being implemented and tested.

\subsection{Two-Leg Test Platform: BILL-Ant-p2}

The initial Two-Leg Test Platform (Fig. 6.1) is a mock-up of the two front legs from BILL-Ant-p and was used during the development of that hexapod as well. During development of the new hexapod, the Two-Leg Test Platform was dubbed BILL-Ant-p2 ('p2' both for 'prototype \#2' and 'two-legged prototype'). To start, only one of the legs from the platform was used, but the other leg was added within the week.

This step was critical to the evaluation of the SCASM method of intra-leg joint control. By initially limiting the number of legs, the work could focus on generating effective stepping movements, and allowed other concerns of a full hexapod (such as posture control) to be left for later. 


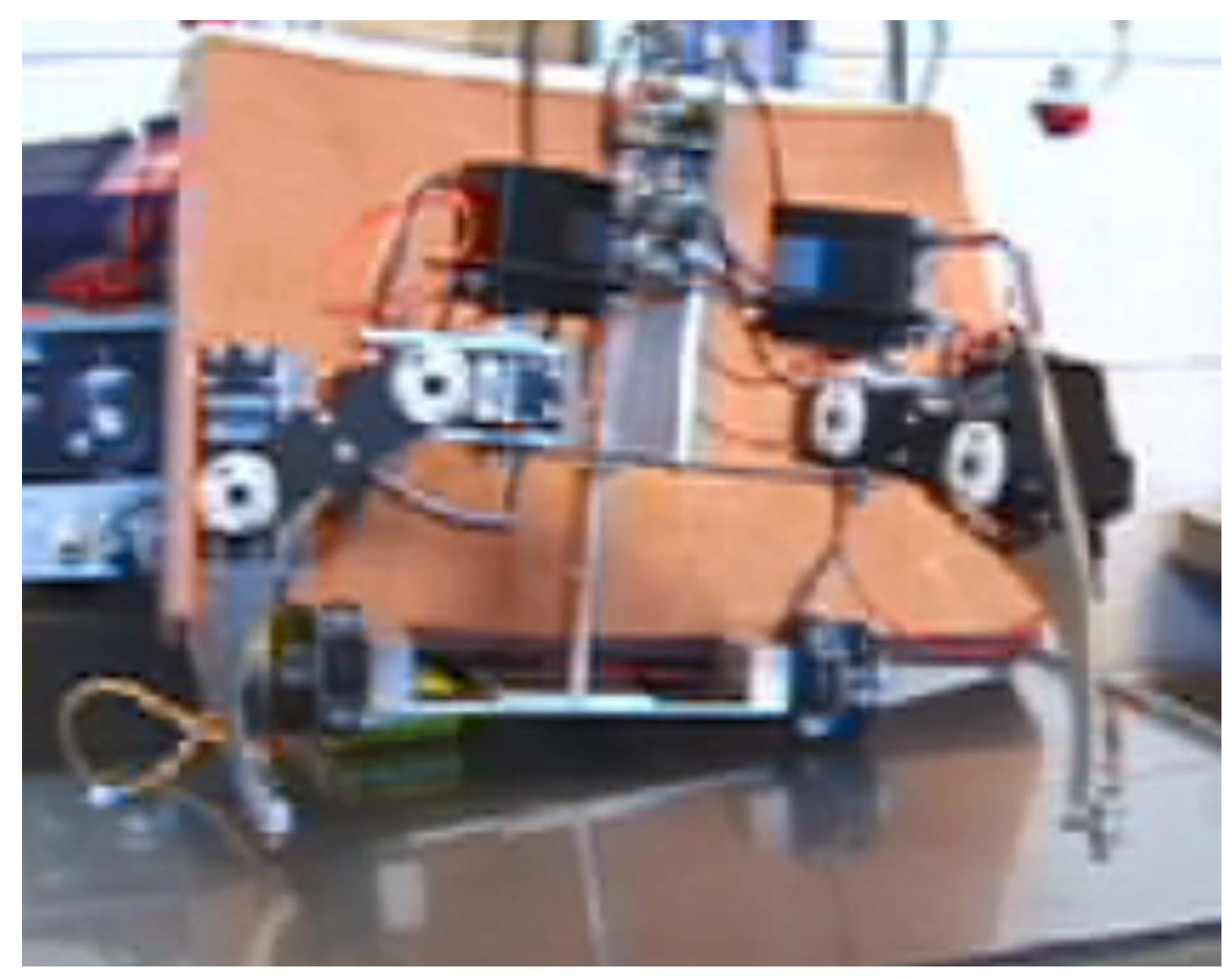

Figure 6.1. Early version of the Two-Leg Test Platform.

\section{This version of the Two-Leg Test Platform used momentary push-button switches to detect ground contact.}

Work with BILL-Ant-p2 began with the original form of SCASM (without the reflex pathways), in order to start more simply. Two BrainStem microcontrollers, one for each leg, were used at this stage. The hobby servo motors within the leg were modified to allow the microcontroller to read the position information from the servo's internal potentiometer.

While minimally effective, the behavior of hobby servo motors assures that the motor will be positioned at the commanded location, so this feedback loop was removed when implemented on the hexapod. Given a faster controller and more 
accurate position information, smooth stepping with active compliance could be performed.

Sensing of the motor current loads was not easily possible with these motors due to extremely noisy sensory output signals. As a result, the foot-mounted FSRs and obstacle contact switches were used to sense interactions with the environment. The ability to accurately read the current signals would allow the two 'external' sensors to be removed. This would eliminate unnecessary wiring and improve the appearance of the leg.

\subsubsection{Simulation of SCASM and BILL-LEGS}

When using simple hardware and simple software, testing with a physical model yields identifiable results with real-world physics. However, there are often times when a simulation is helpful, or even necessary. A simple simulation is useful for testing proof-of-concept theories, while more complex simulations with good dynamics engines can allow visualization of what would happen with the physical hardware.

During this research, simple simulations of SCASM and BILL-LEGS were created in MATLAB. Both sets of simulations were conducted to test proof-ofconcept theories quickly, easily, and safety without risk to the hardware. While neither simulation had a physics-based environment, the resulting data was sufficient to prove the theories that modulation of SCASM parameters can promote turning and that the BILL-LEGS gait generation method could produce 
stable, speed-dependent gaits. This information was sufficient to proceed with implementation on the real hardware, which was successful when testing the basic intra-leg and inter-leg control methods.

\subsubsection{BILL-Ant-p2 with SCASM, BILL-LEGS, Reflexes, and Goal Seeking}

In order to show that all of the major control systems could work together in a small scale environment, BILL-Ant-p2 was upgraded (Fig. 4.4) to include all of the functionality expected of BILL-Ant-a. This included: the modified version of SCASM with the additional sensory pathways that allowed reflex behaviors (Fig. 4.12); the BILL-LEGS gait generation method (although only two legs were being tested); and the addition of the "head" controller, neck motor, and stereoscopic photo sensors, which allowed goal seeking and turning behaviors. The incorporation and integration of all of these systems provided the ability to test how the systems performed when acting simultaneously.

As BILL-Ant-p2 is a stand-mounted pair of legs, posture control was still not considered at this stage. In keeping with the design philosophy of testing individual pieces and then incrementally adding functionality, this phase of the development was focused on the combination of leg control and modulation methods.

During this phase, BILL-Ant-p2 was separately tested on irregular terrains, to show efficacy of the reflex behaviors, and then with a light-based goal, to test the 
phototaxis and turning behaviors. Fig. 6.2 shows a series of steps taken while running one of the goal seeking experiments.

Once goal seeking tests yielded good results, the full set of behaviors was then tested. At this time, the platform was exercised by leading it through a lightly cluttered environment (testing reflexes) using a flashlight as the light-based goal (testing goal seeking and turning).
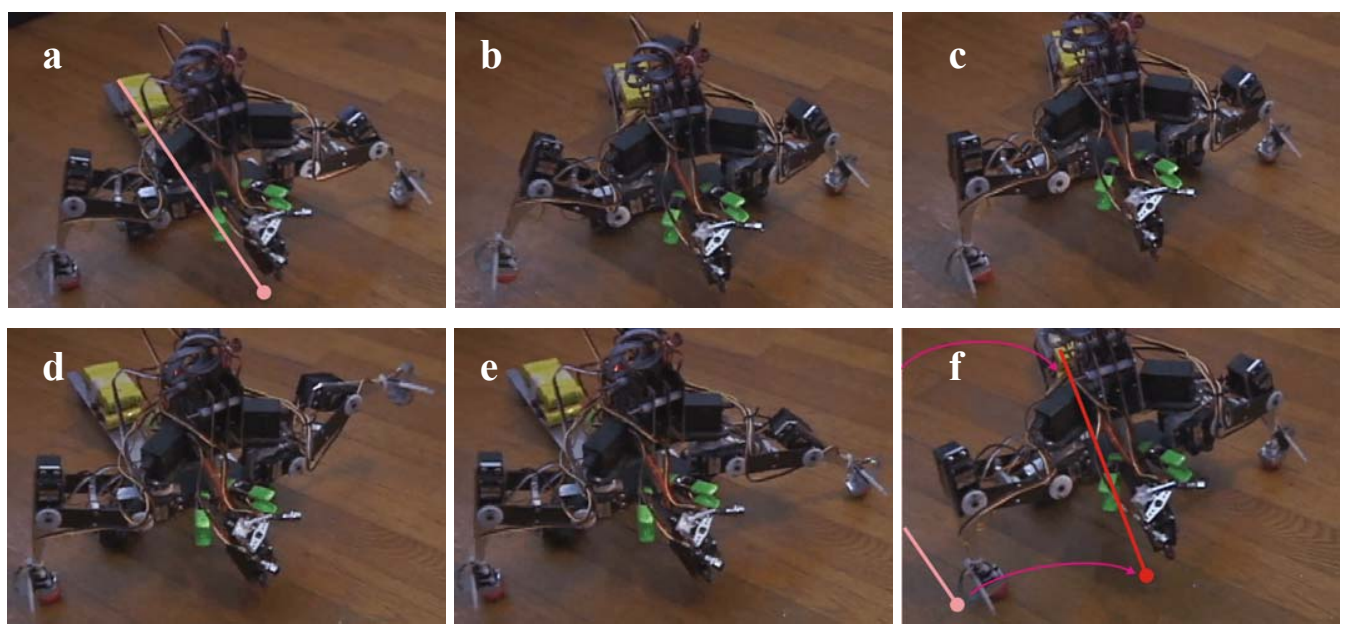

Figure 6.2. Series of images showing the fourth version of the Two-Leg Test Platform following a light source located toward its left.

The series of images shows the neck turning toward the left as the test platform locates a light-based goal (a). The third BrainStem microcontroller modulates the SCASM values on the two leg controllers to cause each of the legs to change their nominal sagittal stepping motions into stepping to the left

(b-f). In the last image, the test platform has displaced itself noticeably toward the goal (f). Note: due to the omni-directional wheels mounted on the base of the platform, the Two-Leg Test Platform executed a lateral displacement, rather than a turn. This is only due to the type of wheels used and the platform having only two legs. Using standard wheels would have resulted in a turning motion. 


\subsection{Six-Legged Hexapod: BILL-Ant-a}

Following the integration of SCASM with reflex behaviors, modulation of SCASM to induce turning, and the incorporation of the BILL-LEGS gait coordination method on the Two-Leg Test Platform, BILL-Ant-p2, work then began on the overhaul and upgrade of the original hexapod (BILL-Ant-p) in order to create BILL-Ant-a. The full network of seven BrainStem microcontrollers replaced the previous servo control board, and obstacle contact switches were added to the legs.

\subsubsection{Leg Range-of-Motion Limits}

Initially, the same software and parameters used with BILL-Ant-p2 was used for the legs of BILL-Ant-a, with the exception of the rear leg pair whose behavior is different than that of the other legs (Section 4.2). It was noted, however, that the close proximity of the ThC joints inhibited the desired movement of the legs. This was not an issue with the Two-Leg Test Platform as there were no ipsilateral neighboring legs. To remedy this, range-of-motion limitations were imposed in software in order to avoid collisions between same-side legs.

By adding these RoM limits, adjustments needed to be made to the SCASM parameters, notably the "muscle activations" that controlled joint speeds. The retraction speeds of the ThC joints were reduced to match the shorter step lengths caused by the RoM limits. 


\subsubsection{Replacement of the Right-Rear FSR Sensor}

From years of use beginning with BILL-Ant-p, the output tab of the right-rear FSR sensor was bent too sharply over the edge of the foot plate and the embedded traces became separated. As no replacement sensor was on hand, the FSR was replaced by a momentary push-button switch (Fig. 6.3).

This switch was mounted to the top plate of the foot and a small hole was made in the same plate for the switch plunger to extend through. The bottom plate of the foot assembly was loosely connected to the top plate by its four screws such that ground contact would cause the bottom plate to depress the switch.

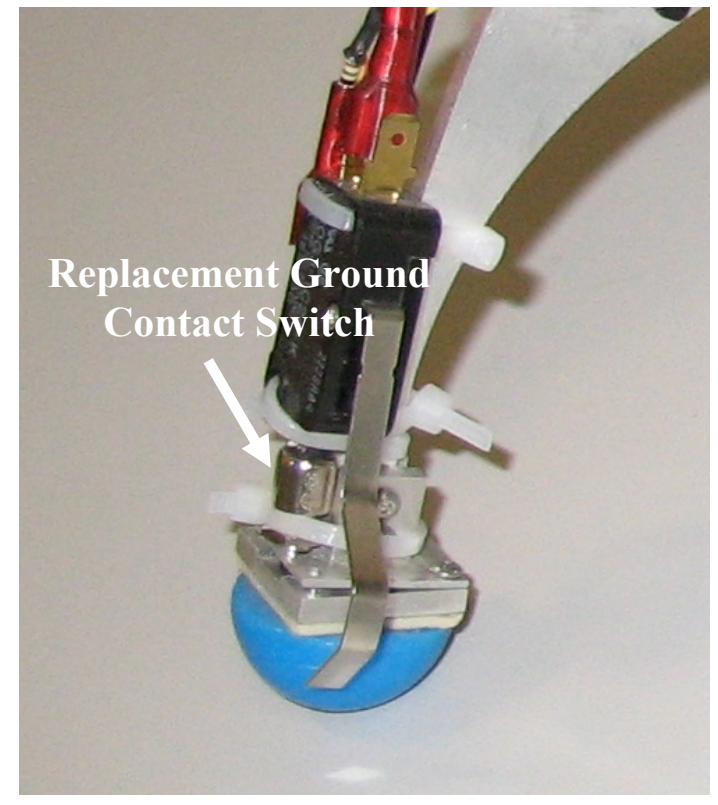

Figure 6.3. Right-rear foot with replacement ground contact switch.

A hole in the upper foot plate allowed the lower foot plate to depress the switch plunger when ground contact is made. 
In the future, consideration must be made for strain relief of sensor tabs, sensor cables, and motor cables, especially on robots like this one with so many moving parts. This will prolong the lives of those elements.

\subsubsection{Alteration of Middle Leg Gait Controllers Creates CPG-Like Behavior}

While it functioned adequately for some time, eventually the new right-rear leg ground contact switch became highly unreliable, detecting ground contact only about two-thirds of the time. This was due mostly to the make-shift replacement of the FSR sensor. Ideally, had one been available, a new FSR should have been installed.

Since the right-rear leg did not reliably detect ground contact, it would not enter its stance phase and commence with retraction. Then, since the leg believed that it was still in its swing phase, the right-middle and left-rear legs would also not step because of the BILL-LEGS Mechanism 1 constraint, which prevents a leg from entering its swing phase so long as at least one neighboring leg was already in swing. The remaining three legs still stepped normally, since their neighbors were reporting their presence in stance phases properly; however, with three legs perpetually on the ground, the robot was unable to make forward progress due to

the friction of the legs in stance. Borrowing again from insect neurobiology, the middle legs were reprogrammed to have a CPG-like gait behavior.

In insects, the oscillatory behavior of joints and legs is affected by sensory inputs. When there is a lack of such inputs, the tonic behavior of the legs is exhibited. 
While not reactive or adaptive, the "open-loop" behavior is sufficient to maintain locomotion. This behavior was dominant for the middle leg pair, however, when sensory signals were occasionally received from the right-rear leg, indicating its swing/stance phase, neighboring legs would adapt their stepping actions accordingly.

\subsubsection{Update of CTr Joint "Muscle Model” to Promote Posture Control}

The Two-Leg Test Platform has a vertical support to maintain its thorax height. BILL-Ant-a relies solely on its legs for this support. As such, a form of posture control was required to maintain a desired ground clearance height.

To implement a basic form of posture control, the CTr joint "muscle model" was modified slightly. The new model had a very aggressive "muscle activation" for when the CTr joint was above a preset standing angle (the initial angle of the $\mathrm{CTr}$ joint when the robot was standing statically at startup). This model allowed muscle modulation to range from $100-200 \%$ of its nominal activation while the joint angle was greater than its typical standing angle (Fig. 4.10, c).

When the CTr joint angle was below the standing angle, the "muscle model" reduced the activation to be between $0-12.5 \%$ of its value. These alterations to the "muscle model" caused the joint to depress heavily when the leg was too high, and very minimally when too low. The result was that BILL-Ant-a had a form of virtual attractor for each of the legs that caused them to maintain the body at a desired distance from the ground. 


\subsubsection{Goal Searching Behavior for When a Goal Is Not In View}

The original phototransistor sensor assembly relied on alligator clip to hold the sensors in place. This provided a wide field-of-view (approximately 120deg), however the weak gripping forces of the clips were often overcome by the forces acting on the sensors by the sensor cables. The solution to this problem was a new sensor mounting bracket (Fig. 4.22).

The new sensor bracket held the phototransistors firmly in place; however, the sensors no longer had a splayed orientation. This reduced the field-of-view to only $45 \mathrm{deg}$. While the goal seeking behavior that actuated the neck to track lightbased goals still operated as before, the greatly reduced field-of-view meant that goals were not seen unless very close to the centerline of the robot. A solution, as is observed in biological systems (Bell 1990), was to implement a goal searching, or "scanning", behavior.

The searching behavior was programmed into the main program residing on the "head" microcontroller and was based on a light sensing threshold. When the sensed light levels were above the threshold, this indicted that the phototransistors had located a goal, and the normal goal seeking behavior proceeded. When the light levels were below this threshold, a searching behavior was performed.

The searching behavior began with reducing the leg speeds to zero, such that the robot stopped making forward progress. Next, the neck servo motor was oscillated through it range-of-motion indefinitely until the light threshold level 
was reached. By oscillating the neck over its $\pm 45 \mathrm{deg}$ RoM, the effective field of view was increased from $45 \mathrm{deg}$ to $135 \mathrm{deg}$, thereby increasing the chances of locating a goal. Once the threshold was exceeded, full speed was restored and BILL-Ant-a progressed toward the goal.

\subsubsection{Update to BILL-Ant-a Goal-Seeking-Based Speed Control}

As an addition to the goal searching behavior, light level-based speed control was introduced. When the light-based goal was not observed, the robot's speed was set to zero. Once the goal was seen, full speed resumed. However, when the sensed light levels exceeded an upper threshold, indicating that the robot had neared the goal, the speed was tapered off as the light levels increased, reducing to zero when the goal was reached.

These last two modifications provided a good deal of robustness and adaptability to the goal seeking behavior, and allowed for easier one-man video recording of the robot in motion, since the robot would not walk when the goal could not be observed. 


\section{CHAPTER VII}

\section{SYSTEM PERFORMANCE}

\subsection{General}

This chapter quantifies the robot component weight characteristics, speed and turning performance, ability to navigate slightly- and highly-irregular terrain, and performance of goal seeking behaviors.

7.1 BILL-Ant-a System, Subsystem, and Component Masses

The robot mass is $5.79 \mathrm{lbs}(2.63 \mathrm{~kg})$. System, subsystem, and component masses were weighed on a scale with $1.0 \mathrm{~g}$ resolution. Ounce values were calculated from the gram masses. Both are listed below in Table 7.1.

\begin{tabular}{|c|ccc|}
\hline Component or Subsystem & \multicolumn{3}{|c|}{$\begin{array}{c}\text { Mass } \\
\text { (oz./g) }\end{array}$} \\
\hline BILL-Ant-a Total Mass & \multicolumn{3}{|c|}{} \\
\hline Body Section & 100.32 & $/$ & 2850 \\
\hline Top Plate & 5.81 & $/$ & 165 \\
\hline Bottom Plate & 1.58 & $/$ & 45 \\
\hline Spine & 1.58 & $/$ & 45 \\
\hline Front Plate & 1.62 & $/$ & 46 \\
\hline Rear Plate & 0.46 & $/$ & 13 \\
\hline Fasteners and misc. & 0.46 & $/$ & 13 \\
\hline Leg (x6) & 0.11 & $/$ & 3 \\
\hline Coxa & 9.64 & $/$ & 274 \\
\hline Femur & 0.77 & $/$ & 22 \\
\hline Front Plate & 0.63 & $/$ & 18 \\
\hline & 0.28 & $/$ & 8 \\
\hline
\end{tabular}




\begin{tabular}{|c|ccc|}
\hline Rear Plate & 0.18 & $/$ & 5 \\
\hline Yoke Strut & 0.18 & $/$ & 5 \\
\hline Tibia & 0.39 & $/$ & 11 \\
\hline Foot & 0.49 & $/$ & 14 \\
\hline Obstacle Contact Switch & 0.32 & $/$ & 9 \\
\hline R/C Servo (x3) & 2.25 & $/$ & 64 \\
\hline Fasteners and misc. & 0.28 & $/$ & 8 \\
\hline BrainStem (x6) & 0.74 & $/$ & 21 \\
\hline Power System & 22.74 & $/$ & 646 \\
\hline Battery (x4) & 3.31 & $/$ & 94 \\
\hline Power Converter (x4) & 0.63 & $/$ & 18 \\
\hline Terminal Block and Power Cables & 4.65 & $/$ & 132 \\
\hline $\begin{array}{l}\text { Switches, Holders, Fuse, Fasteners } \\
\text { and misc. }\end{array}$ & 2.32 & $/$ & 66 \\
\hline "Head" and Neck Components & 5.49 & $/$ & 156 \\
\hline BrainStem (x1) & 0.74 & $/$ & 21 \\
\hline Body-neck Mount & 0.95 & $/$ & 27 \\
\hline R/C Servo (x1) & 2.25 & $/$ & 64 \\
\hline Stereo Phototransistor Assembly & 1.13 & $/$ & 32 \\
\hline Fasteners and misc. & 0.42 & $/$ \\
\hline Robot-wide Fasteners and misc. & 3.98 & 12 \\
\hline
\end{tabular}

Table 7.1. BILL-Ant-a component and subsystem masses.

\subsection{BILL-Ant-a Motor Current Consumption}

BILL-Ant-a was powered by $6.0 \mathrm{vdc}$ supplies (either on-board batteries or an offboard bench top supply). Battery power lasted at most $36 \mathrm{~min}$ when walking on level surfaces. Activities such as flat surface walking with surmountable obstacles reduced battery life to about 30min, while navigating the irregular terrain field reduced battery life to only $10-12 \mathrm{~min}$. 
For all activities, the current for the BrainStem logic power remained relatively constant at 0.40A. Motor current for several tasks are listed below in Table 7.2.

\begin{tabular}{|l|c|}
\hline \multicolumn{1}{|c|}{ Activity } & \multicolumn{1}{|c|}{$\begin{array}{c}\text { Motor Current } \\
\text { (typical Amperes) }\end{array}$} \\
\hline $\begin{array}{l}\text { Resting on support stand } \\
\text { (legs not in contact with ground) }\end{array}$ & $\begin{array}{c}0.40 \\
\text { (range: } 0.35-0.75)\end{array}$ \\
\hline $\begin{array}{l}\text { Standing } \\
\text { (after being placed on flat ground) }\end{array}$ & 1.20 \\
\hline $\begin{array}{l}\text { Standing } \\
\text { (performing a standing action) }\end{array}$ & 2.70 \\
\hline $\begin{array}{l}\text { Walking } \\
\text { (on flat ground) }\end{array}$ & 5.50 \\
\hline $\begin{array}{l}\text { Walking } \\
\text { (on slightly irregular terrain) }\end{array}$ & $\begin{array}{c}7.80 \\
\text { (range: } 6.50-9.00)\end{array}$ \\
\hline Climbing Irregular Terrain & 9.20 \\
\hline (range: $7.30-13.60)$ \\
\hline
\end{tabular}

Table 7.2. BILL-Ant-a motor current consumption for various tasks.

\subsection{BILL-Ant-p2 on Flat Terrain}

The following sections describe the performance of the Two-Leg Test Platform (BILL-Ant-p2) while operating on flat ground with few or no obstacles.

\subsubsection{BILL-Ant-p2 Basic Walking}

When walking on flat ground with no obstacles, the Two-Leg Test Platform was able to achieve a maximum walking speed of $2.4 \mathrm{~cm} / \mathrm{s}$. While the servo motors are capable of faster performance, the program demands on the BrainStem microcontrollers, which reduced the computation cycle speeds to only $6-10 \mathrm{~Hz}$, 
prevented this. When attempting to operate at faster motor speeds, the leg joints would greatly overshoot their respective SCASM state transition threshold setpoints, because of the slow operating speed of the microcontrollers, and the stepping motion would deteriorate to uselessness.

In order to quantify the stepping motions, data logging of the joint angles and joint direction states was recorded. Due to the high overhead associated with how RS-232 communications is implemented with the BrainStems, fetching data from the microcontroller network would alter the walking behavior (joints would slightly overshoot their respective state transition threshold setpoints due to slower processing cycle speeds created by the communication demands). To minimize this, "muscle activation" values were all reduced proportionately when data logging occurred. Fig. 7.1 shows a foot path in the sagittal plane and joint state data during a stepping motion. This information was recorded from the right leg during a longer-than-normal step length of about 6.7 in $(17.0 \mathrm{~cm})$. Typical step lengths are approximately $3.5 \mathrm{in}(8.9 \mathrm{~cm})$.

Data recorded from a sequence of three steps shows the joint angle positions and ground contact status for both left and right legs (Fig. 7.2). From these graphs, the following can be noted. It can be seen that the swing phase is very short when compared to the stance phase (data sections where the ground contact graphs are 0 ), ranging from $1 / 6$ to $1 / 2$ of the duration of the stance phase, yet remaining relatively constant in duration between successive steps. The change in slope seen in the ThC joint angle graphs shows the difference between the higher 

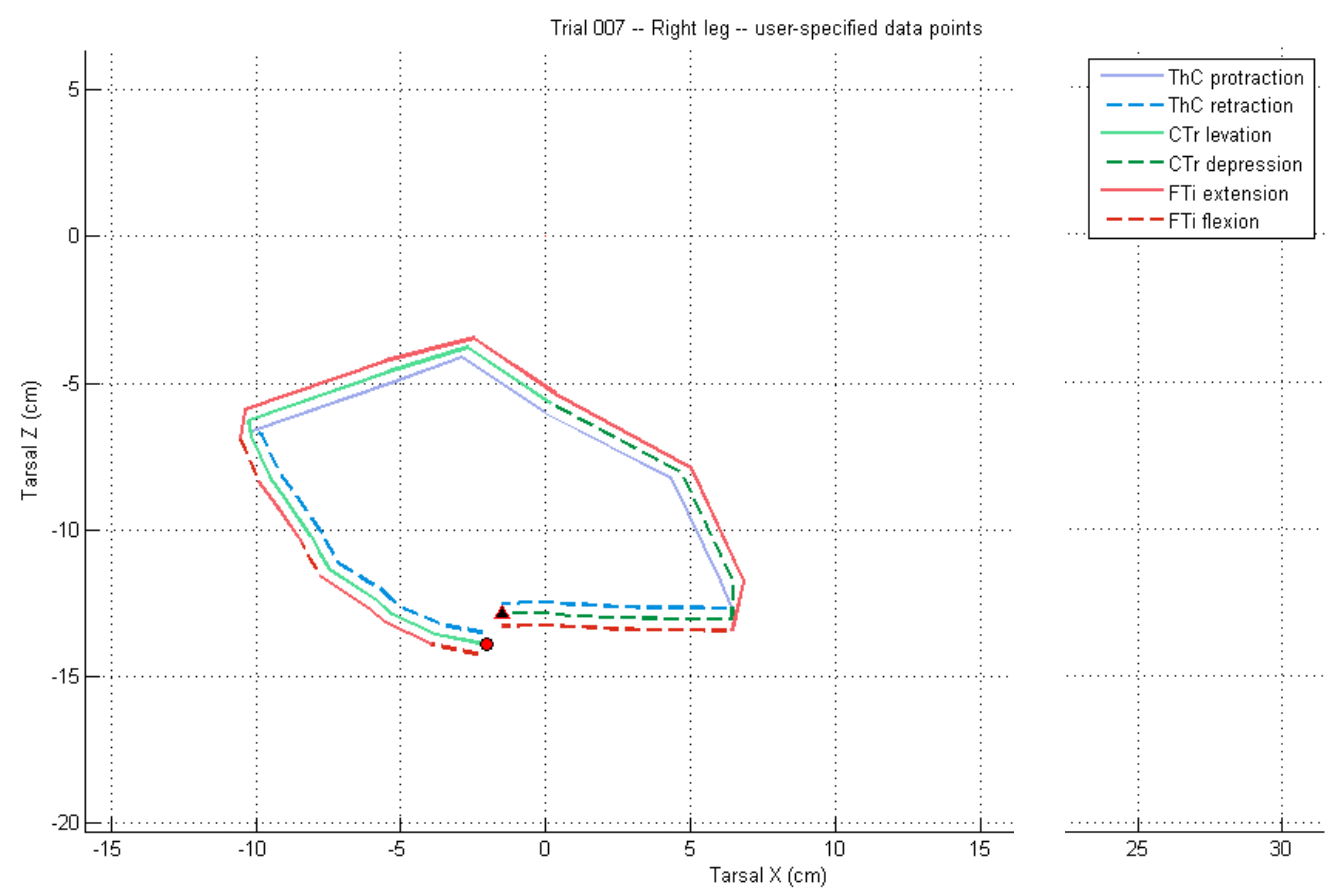

Figure 7.1. BILL-Ant-p2 right leg typical stepping motion foot path.

\section{The $\mathrm{x}$-axis is shown relative to the coxa location; the $\mathrm{z}$-axis is shown relative to the coxa mounting point on the thorax.}

protraction and lower retraction "muscle activation" values, and the tapering of the slopes near the swing/stance and stance/swing phase transition boundaries is due to the "muscle model" reducing the joint torque as the joint nears its rangesof-motion. The CTr joint angle graphs lead the ground contact graphs. This is due to the leg needing to levate for a period of time in order to unload the leg before the foot is actually off the ground. The condition where the FTi joint is in extension during the swing phase and then enters flexion once ground contact is restored can also be observed. Another key point to note is that the steps are each unique. This is due to the emergent formation of the stepping motion, rather than having stepping created by a fixed pattern generator, or even a regular-shaped pattern cycle that may get modified by interactions with the environment. 

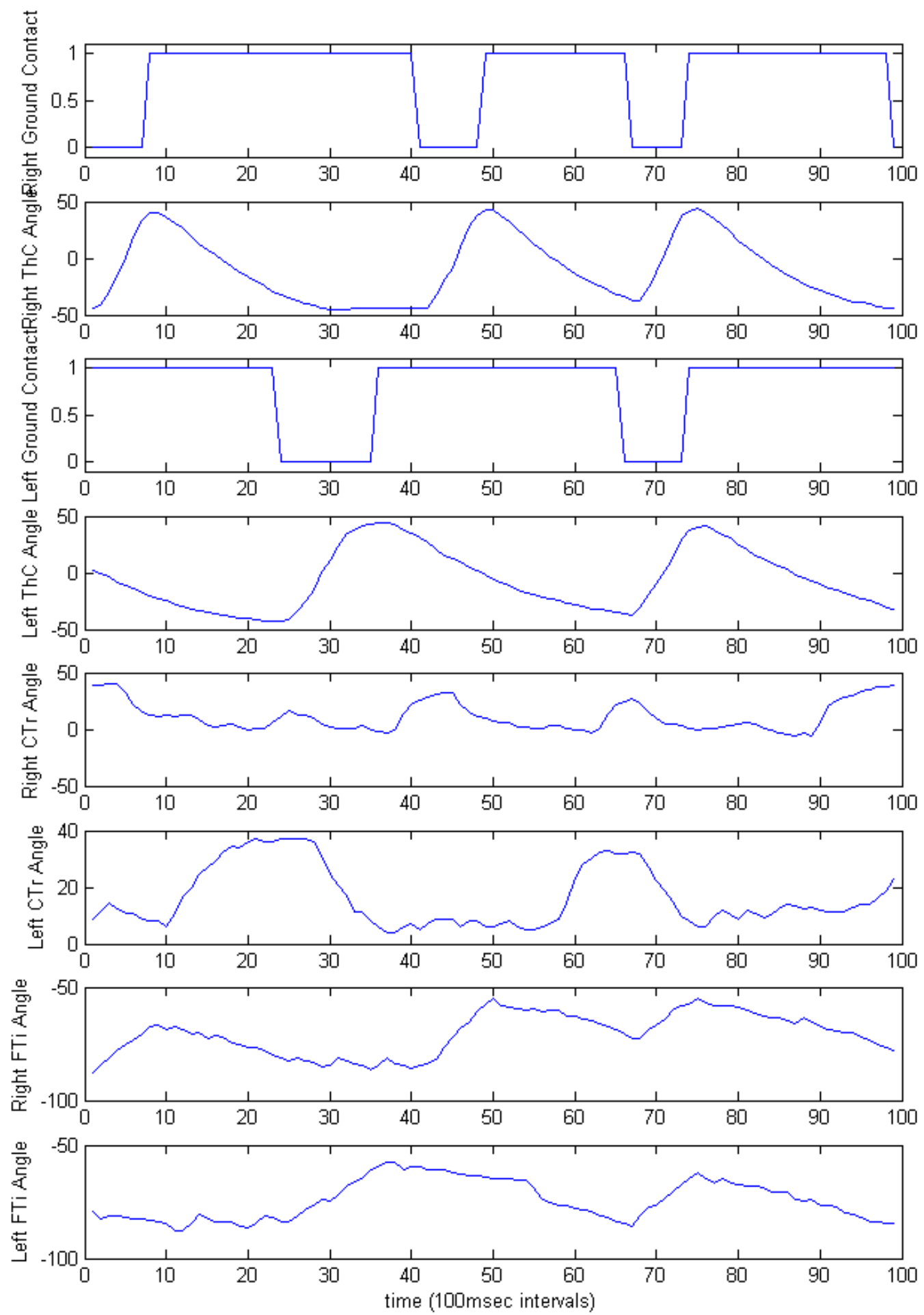

Figure 7.2. BILL-Ant-p2 leg joint angles and ground contact states.

Swing phases for each leg occurred when the respective ground contact graph values are 0. 


\subsubsection{BILL-Ant-p2 Reflex Performance on Cluttered Terrain}

While still on flat ground, obstacles were placed in the path of BILL-Ant-p2. The searching reflex was tested by having the Two-Leg Test Platform walk on a raised surface with a variable width gap in front of the right foot. Elevator reflex trials were conducted by placing objects of various heights in the path of the right foot.

\subsubsection{Searching Reflex}

The nominal step length for BILL-Ant-p2 is approximately $3.5 \mathrm{in}(8.89 \mathrm{~cm})$, suggesting that a $3.5 \mathrm{in}(8.89 \mathrm{~cm})$ wide gap would be possible to cross without notice, provided that the foot landed just at the edge prior to commencing its swing phase. In practice, however, this is almost never the case, and so the searching reflex would be triggered to cross the gap when ground contact was not achieved when expected.

Since the searching reflex extends the step forward incrementally, through iterations of levation/retraction and depression/protraction while advancing the TC_DEP state transition threshold setpoint (see Section 4.4.1 for additional details), the overall step length can be slightly longer than the nominal. Fig. 7.3 shows a sequence of images while the right leg of BILL-Ant-p2 was performing the searching reflex. It can be seen that with each searching reflex iteration, the step extends slightly forward (due to the TC_DEP setpoint moving) and slightly downward (due to the CTr joint angle searching reflex trigger threshold being lowered with reflex action before ground contact is re-established). 

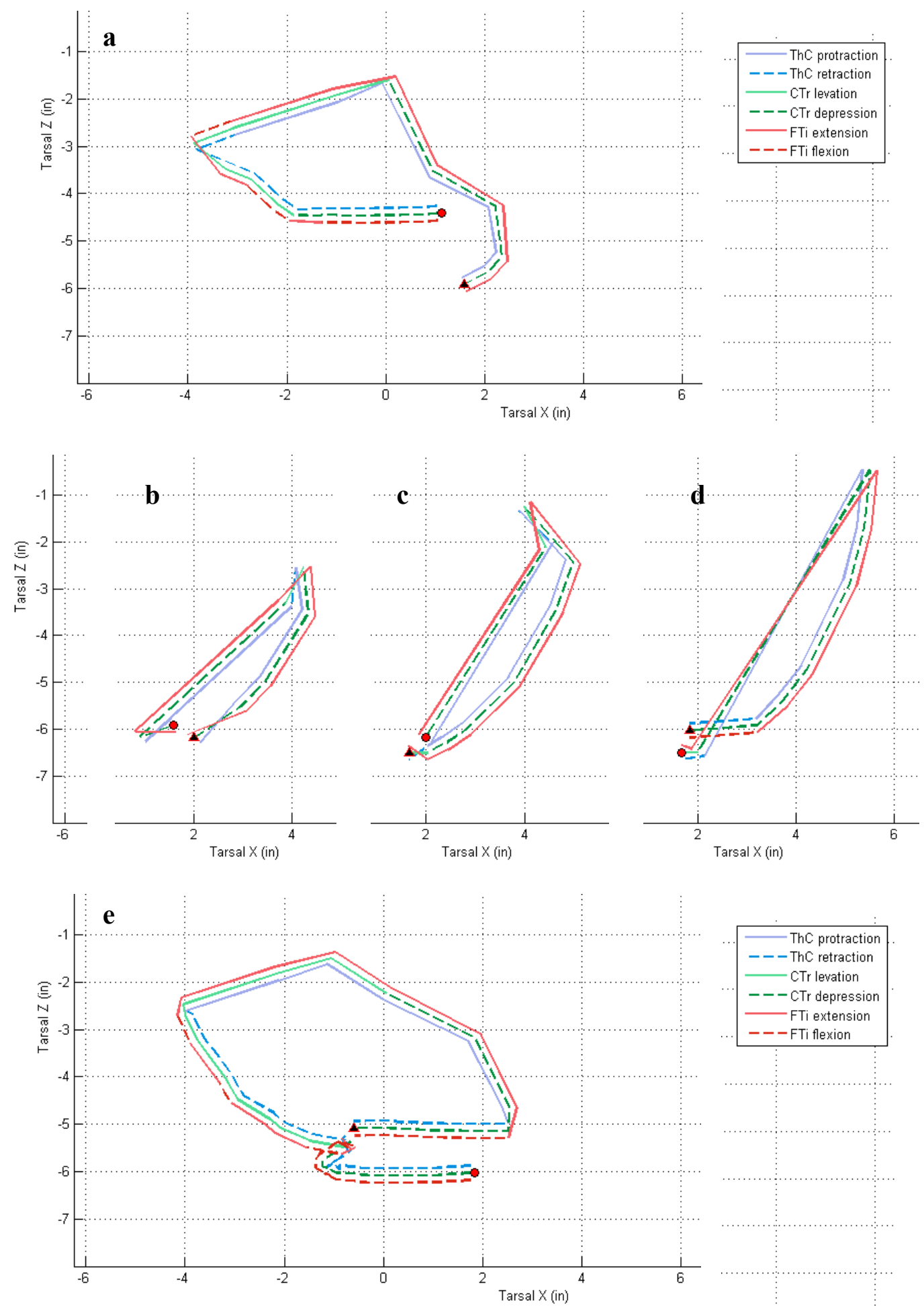

Figure 7.3. BILL-Ant-p2 right-leg foot path during a searching reflex.

Three iterations of searching reflex $(b, c, d)$ were performed before ground contact was re-established. The new ground level was discovered $0.6 \mathrm{in}$ $(1.5 \mathrm{~cm})$ below the previous level (e). 
Experimentally, it was observed that the Two-Leg Test Platform was reliably able to cross gaps of up to $2.5 \mathrm{in}(6.35 \mathrm{~cm})$. Larger gaps of up to 3.0in $(7.62 \mathrm{~cm})$ were passable only during some of the trials, depending on the location of the foot relative to the gap edge prior to initiating the searching reflex, and also due to the ground height of the far side of the gap (with higher ground heights leading to more success).

\subsubsection{Elevator Reflex}

BILL-Ant-p2 has a nominal step height of about $1.25 \mathrm{in}(3.18 \mathrm{~cm})$. Obstacle heights above this level trigger the elevator reflex. Through a series of trials, it was seen that the Two-Leg Test Platform was reliably able to surmount obstacles up to $2.5 \mathrm{in}(6.35 \mathrm{~cm})$ tall. This limit was brought about by the physical range-ofmotion of the CTr joint to levate the leg, and the amount of extension the FTi joint experienced during swing. While the $\mathrm{CTr}$ joint $\mathrm{RoM}$ is fixed, greater extension of the FTi allowed taller obstacles to be surmounted, however, the longer moment arm of the leg in that pose presented a much greater work load to the $\mathrm{CTr}$ joint during depression, when the mass of the test platform needed to be lifted.

Additionally, the higher extension of the FTi only slightly improved on the obstacle height that could be surmounted (clearing heights of up to approximately $2.75 \mathrm{in}(6.99 \mathrm{~cm}))$, while at the same time sacrificing the shape of the foot path in stance. This was due to an excessive flexion action being required to return the leg to an appropriate pose prior to entering swing. The result of this high flexion 
action caused the foot to make a sharp turn while on the ground (the foot path became an obtuse angle with a well-defined cusp, instead of a nearly-straight line). This both upset the smoothness of the foot path and caused increased loading for the FTi joint in the contralateral leg. Due to these negative aspects, the FTi extension activation was kept at a reasonable level.

\subsubsection{BILL-Ant-p2 Modulated Walking with Goal Seeking}

Ten experiments were conducted (five of those being video recoded) with the Two-Leg Test Platform involving locating and moving toward a light source. With the original stereo light sensing assembly, where the phototransistors were held at a splayed angle of approximately 45deg, BILL-Ant-p2 was able to detect light-based goals over a range of $\pm 60 \mathrm{deg}$ from the sagittal plane.

Due to the rear of the test platform being equipped with omni-directional wheels, however, lateral displacement maneuvers, rather than turning maneuvers, were performed. As a result, BILL-Ant-p2 had some trouble reaching light targets that were nearby and required more drastic heading changes. More distant targets, however, were approached with oblique headings that did result in reaching the goal.

7.4 BILL-Ant-a on Flat and Limited Height Variation Terrain

With its stepping motions limited due to the physical mounting locations of neighboring legs, BILL-Ant-a has nominal step length of $2.0 \mathrm{in}-3.0 \mathrm{in}(5.08 \mathrm{~cm}-$ 
$7.62 \mathrm{~cm}$ ), with the middle legs having a shorter step length, and a nominal step height of $0.5 \mathrm{in}(1.27 \mathrm{~cm})$.

\subsubsection{BILL-Ant-a Basic Walking}

BILL-Ant-a has a maximum walking speed on a flat surface of $3.0 \mathrm{~cm} / \mathrm{s}$, which is slightly faster than the Two-Leg Test Platform only due to minor changes in the various stepping parameters, such as the shorter step lengths and different “muscle activation" values.

It should be noted that even with the low computation capable BrainStem microcontrollers, both the Two-Leg Test Platform and the BILL-Ant-a hexapod have faster walking speeds that the previous BILL-Ant-p hexapod, whose maximum speed was a mere $0.4 \mathrm{~cm} / \mathrm{s}$. This is due mostly to having local, onboard controllers, instead of the off-board computer and the serial cable connection used with BILL-Ant-p. The computational simplicity of the new leg control method, coupled with the elimination of the communications bottleneck created by the serial cable aided in the improved performance of the newer robots. With more capable microcontrollers, even faster walking would be possible since joint speeds could be greatly increased without fear of overshooting state transition threshold setpoints due to slow computation times.

While more difficult to gather data than with the Two-Leg Test Platform because of the higher microcontroller processing demands, it was possible during early 
walking trials to record foot path data from the front two legs of BILL-Ant-a (Fig.

7.4).
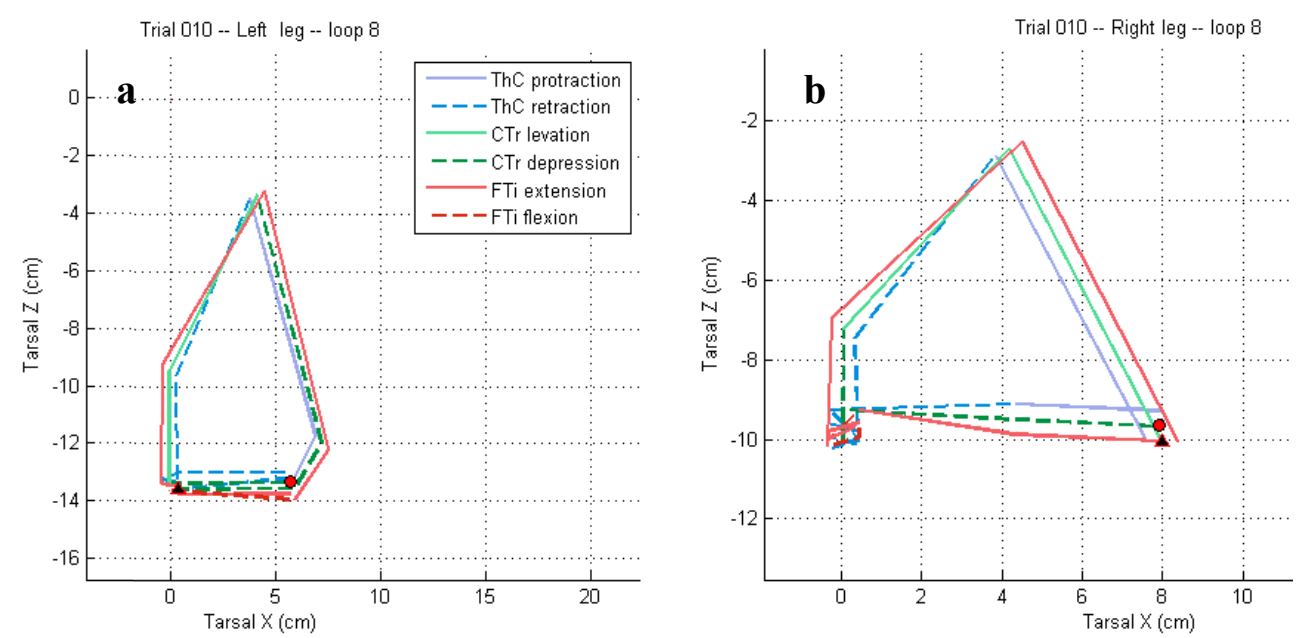

Figure 7.4. BILL-Ant-a left-front and right-front foot paths while walking on a flat surface.

Single stepping motions for the left-front (a) and right-front (b) legs of BILLAnt-a during sequential right-left steps.

Note: the step shapes appear different only due to a difference in the $x$-axis scales. Both steps lengths are approximately 3.15 in $(8.0 \mathrm{~cm})$ long.

7.4.2 BILL-Ant-a Reflex Performance on Flat Terrain with Obstacles

Two mostly-flat test environments were created to test the searching and elevator reflexes.

\subsubsection{Searching Reflex}

The searching reflex was tested by creating a gap across the robot's path that was approximately 2.36in $(6.0 \mathrm{~cm})$ deep and 2.17in $(5.5 \mathrm{~cm})$ across (Fig. 7.5). This 
obstacle presented some difficulty for BILL-Ant-a as the robot's normal step length is about 3.0in $(7.62 \mathrm{~cm})$. The width of the gap was not crossed in all test runs, however, due to the robot being unable reach the far side, based on where the last step ended on the near side. This gap crossing difficulty was only seen with the middle legs. Because of the limited available range-of-motion due to interference with the front and rear legs, the middle legs do not have the ability to advance the TC_DEP threshold forward when initiating the searching reflex. As a result, the step length of the middle legs is not extended. The success in navigating past a gap is then dependent on the placement of the foot prior to commencing the reflex, and how the other legs may be propelling the body during that time.

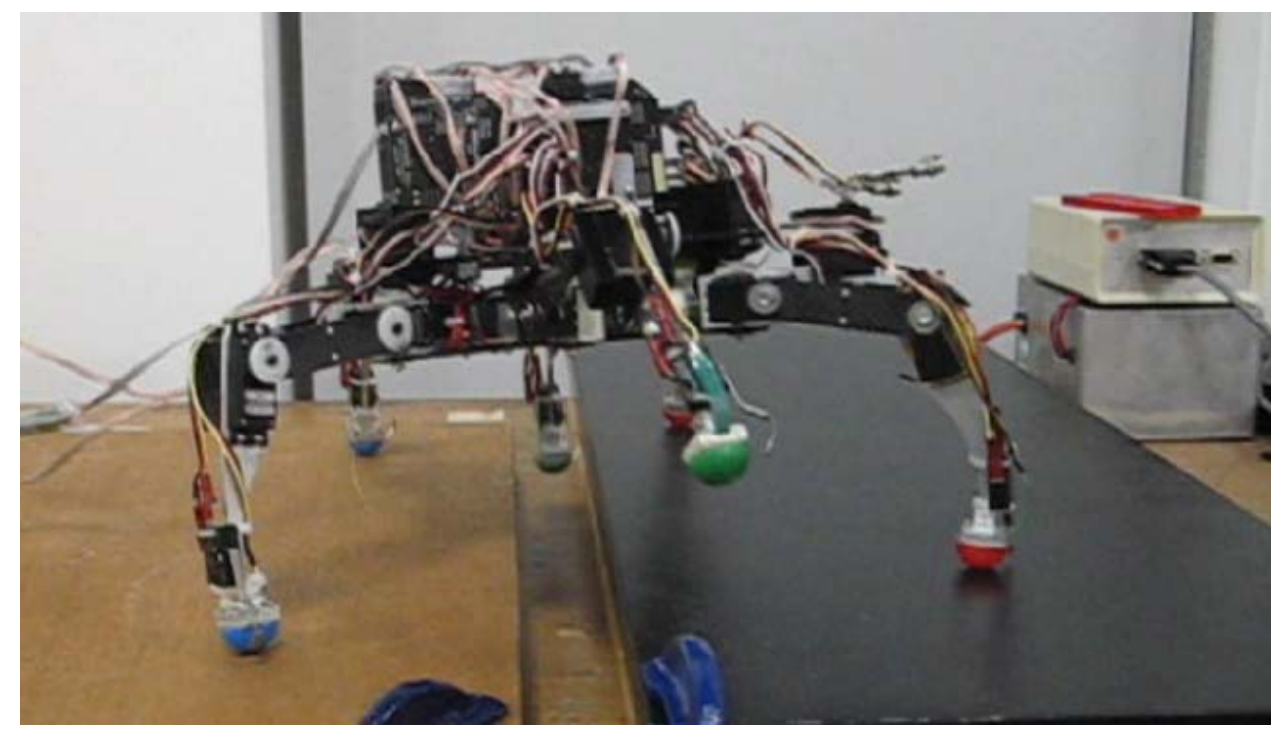

Figure 7.5. BILL-Ant-a gap crossing scenario to test the searching reflex.

Note how the far middle leg is just sensing the gap, initiating the searching reflex, while the near middle leg is concluding the searching reflex with a high, far-forward step toward solid ground beyond the gap. 
When the gap was narrowed to $1.75 \mathrm{in}(4.5 \mathrm{~cm})$, the robot frequently stepped over the obstacle without initiating the reflex. Twice during testing, one of the feet got caught on the edge of the gap and caused the robot to tip forward onto its neck. Despite the few issues concerning the middle legs, during the majority of the trials where the gap width was set to $2.0 \mathrm{in}(5.08 \mathrm{~cm})$, the robot repeatedly demonstrated that the implementation of the reflex was successful.

\subsubsection{Elevator Reflex}

For the elevator reflex, BILL-Ant-a was presented with two obstacles for different tests. The first was a wood beam with a height of approximately 2.17 in $(5.5 \mathrm{~cm})$ and the second obstacle was approximately 0.79 in $(2.0 \mathrm{~cm})$ tall (Fig. 7.6). Given the robot's maximum stepping height of about $2.25 \mathrm{in}(5.72 \mathrm{~cm})$, the robot was barely able to clear the taller obstacle. In a few trials, BILL-Ant-a detected ground contact while the foot was scraping the top of the obstacle. During these trials, the obstacle was stepped onto as a foothold, and then stepped off with the next step. For the cases where the obstacle top was not touched, the beam was cleared and stepped over during a single stepping cycle.

The shorter obstacle, though much lower than the first, was still tall enough to trigger the elevator reflex (given that the robot's normal step height is only $0.5 \mathrm{in}$ $(1.27 \mathrm{~cm}))$ and was easily navigated. Unlike the taller obstacle, the shorter one was stepped over during all of the trials, and the robot did not use the beam as a foothold. 


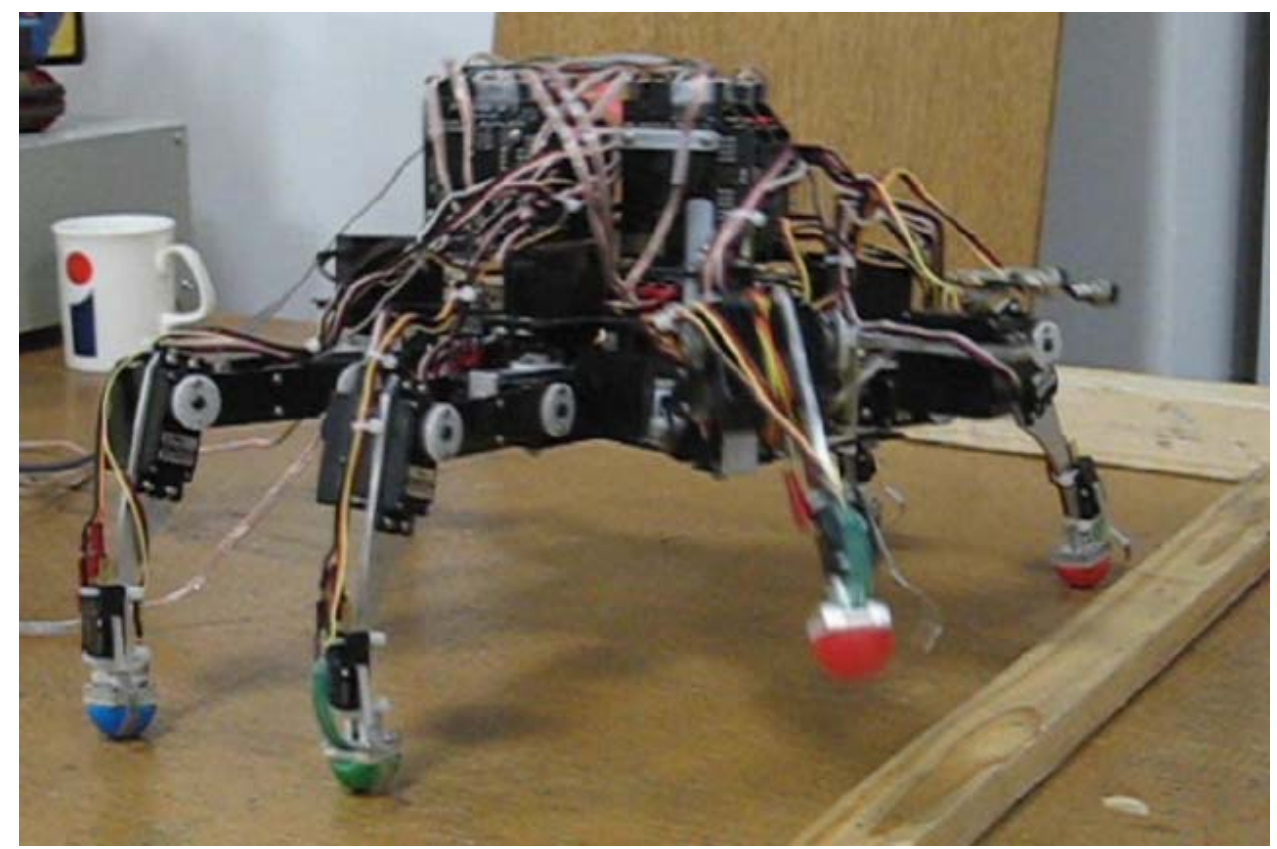

Figure 7.6. BILL-Ant-a performing the elevator reflex.

The image shows the right-front leg in the middle of the reflex behavior. Having just executed the retraction and high levation actions, the leg is preparing to resume the normal stepping behavior with protraction and depression actions.

Below, Fig. 7.7 shows the foot path drawings for the left-front leg during an elevator reflex. Images (a) and (d) show normal stepping motion patterns for the left-front leg of BILL-Ant-a. In (b), the foot collides with an obstruction where the obstacle contact switch is depressed. The elevator reflex, shown in (c) is then triggered where the extreme levation action can be seen. Also shown in (c) is the increase in the step length. This is due, in part, to the advancement of the TC_DEP threshold value, but also because of the high foot height resulting from the large levation action. Because the foot is so high, it takes longer for the depression action to bring the foot back in contact with the ground. During this 
time that the foot is in the air, protraction is persisting, which aids in creating the longer step.

$\mathbf{a}$
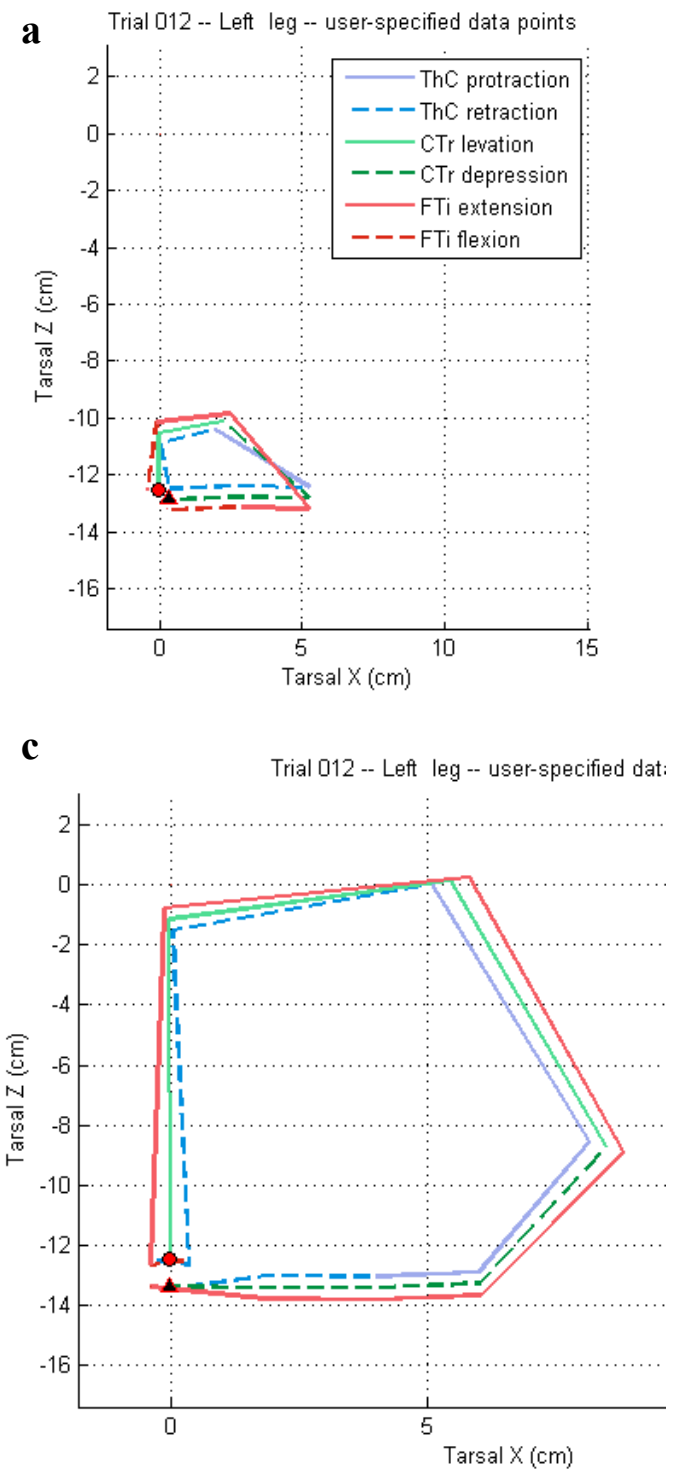

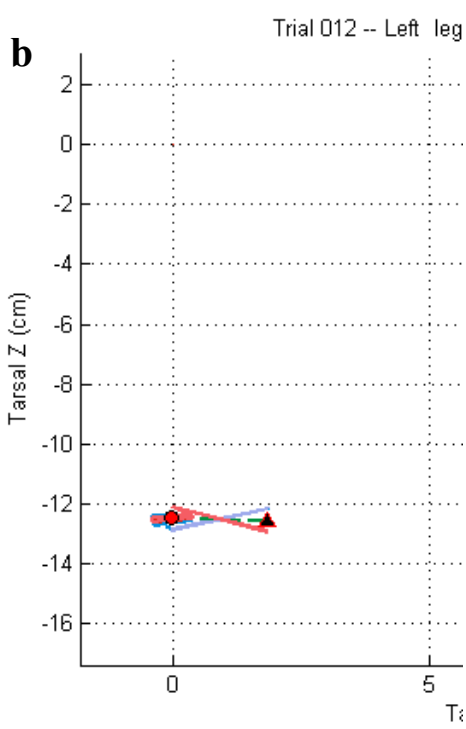

d

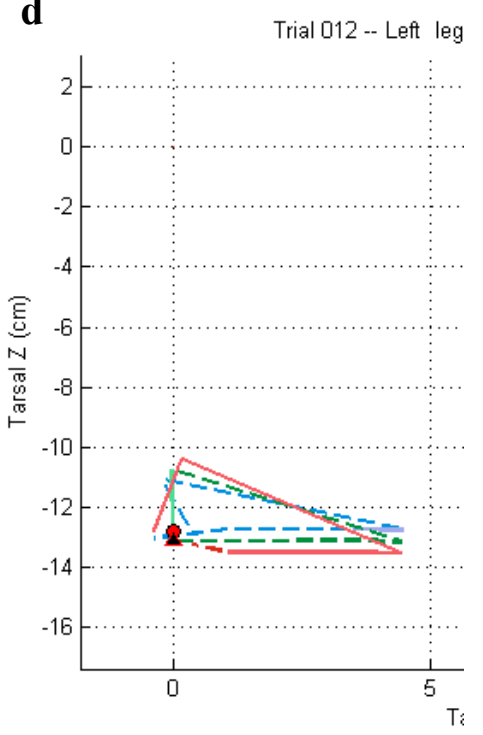

Figure 7.7. BILL-Ant-a right-front foot paths during an elevator reflex.

Image (a) shows a normal, albeit short $(2.0 \mathrm{in}, 5.0 \mathrm{~cm})$ stepping motion. In (b), an obstacle is encountered that stops the forward progress of the foot. (c) shows the elevator reflex behavior with its exaggerated levation action and more-forward-than-normal stepping motion. Finally, in (d) normal stepping motions resume and a small step similar to that in (a) is seen. 


\subsubsection{BILL-Ant-a Modulated Walking and Goal Seeking}

Through several initial trials, BILL-Ant-a was successfully able to locate lightbased goals that were positioned within $\pm 22.5 \mathrm{deg}$ of the sagittal plane. This is a much smaller field of view from that of the Two-Leg Test Platform, since BILLAnt-a has the updated phototransistor bracket that positions the two sensors parallel to one another, instead of the splayed sensor arrangement of the earlier design. With the addition of the goal searching behavior that oscillated the neck left and right by $\pm 45 \mathrm{deg}$ (Fig. 7.8), the field of view was expanded to $135 \mathrm{deg}$.
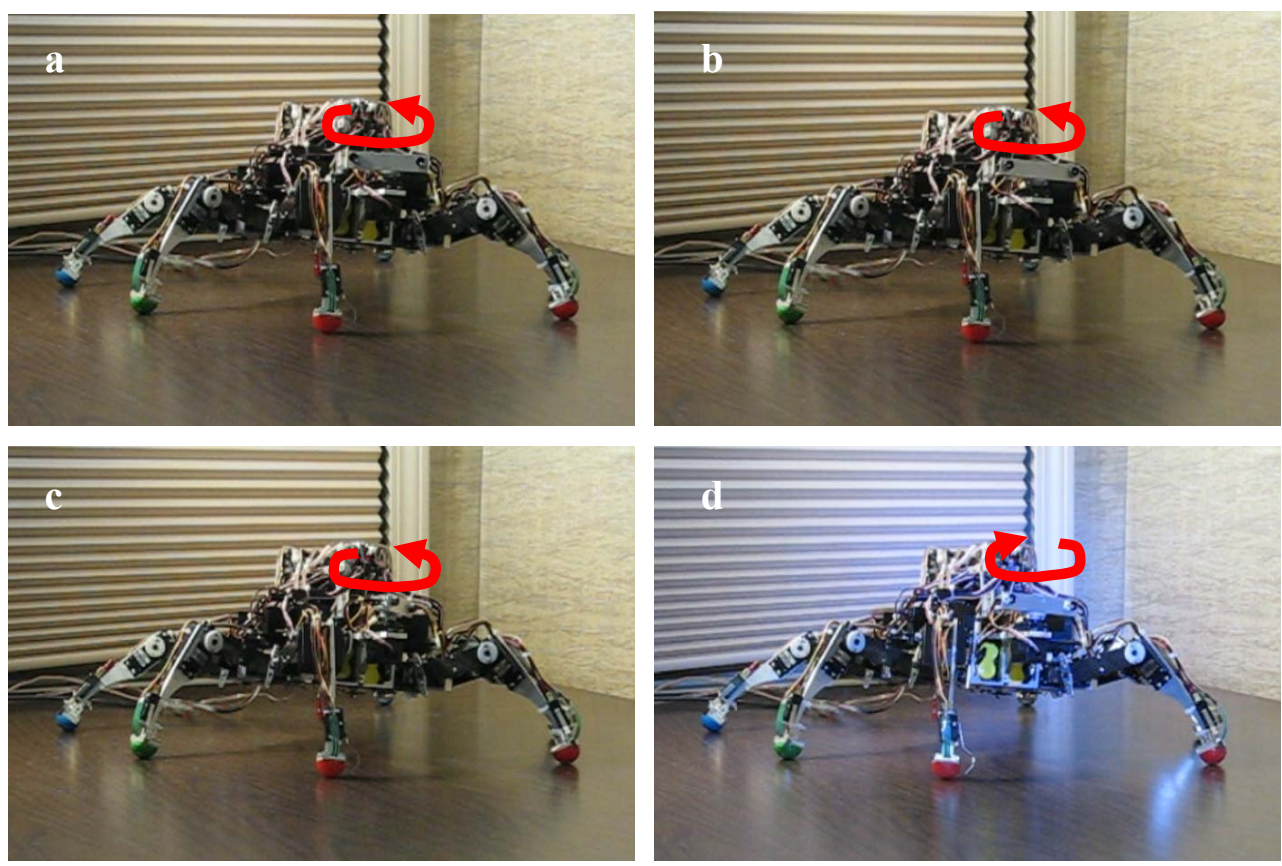

Figure 7.8. BILL-Ant-a performing goal searching behavior.

When the light-based goal is not in view, the neck repeatedly oscillates left and right over a 90deg arc (a, b, c) until the goal is spotted (d). Once the goal is seen, the neck actuates to track the light such that the two phototransistors sense similar light levels. 
While performing the goal-seeking behavior, BILL-Ant-a was able to turn toward the goal with a minimum radius of approximately $22 \mathrm{in}(55.9 \mathrm{~cm})$. Turning was accomplished by each leg having its SCASM setpoints and values modulated by the "head" microcontroller, according to the heading of the neck when the goal was located. Each leg had its values modulated differently, as described in Section 4.5 , in order to produce the turning behavior.

\subsection{BILL-Ant-a in the Random Elevation Field Test Arena}

In order to test the performance of BILL-Ant-a while navigating irregular terrain, a random elevation field test arena was constructed. The arena consisted of two abutting $12.0 \mathrm{in}$ by $24.0 \mathrm{in}$ sections $(30.5 \mathrm{~cm}$ by $61.0 \mathrm{~cm})$ for a total arena size of 24.0 in by 24.0 in $(61.0 \mathrm{~cm}$ by $61.0 \mathrm{~cm})$.

Filling the arena surface was a random assortment of 3.0in by 3.0in foam blocks $(7.62 \mathrm{~cm}$ by $7.62 \mathrm{~cm})$ of varying heights: $0.25 \mathrm{in}(0.64 \mathrm{~cm}), 1.0 \mathrm{in}(2.54 \mathrm{~cm})$, and 2.0in $(5.08 \mathrm{~cm})$. The height of each grid location in the arena was chosen by a software program that was written to randomly select the block height, with the restriction that the total number of each available block could not be exceeded (16 low block, 40 medium blocks, and 40 tall blocks). For the tests conducted in the following sections, the arena grid elevations shown in Fig. 7.9 were used.

Based on the graphical output of the software program, blocks were placed in the arena. Grid values of 0,1 , and 2 corresponded to low, medium, and tall block heights. 
Initially, blocks were placed in the arena and held by friction with neighboring blocks. However, due to issues with the robot dislodging blocks while walking, the foam was eventually glued to the arena floor for better stability.

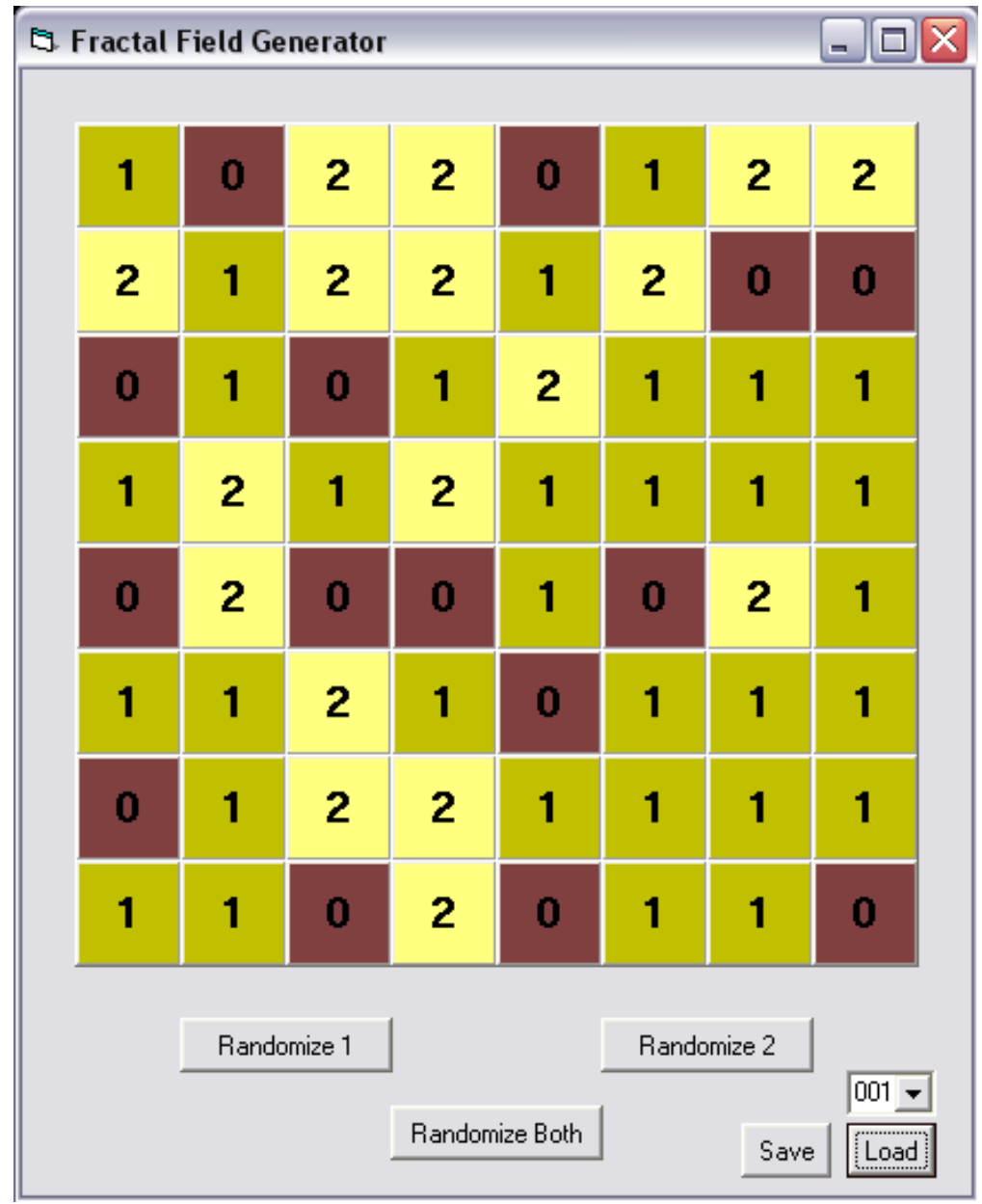

Figure 7.9. Random elevation field test arena layout.

Block labels of 0,1 , and 2 correspond to block heights of 0.25 in $(0.64 \mathrm{~cm})$, $1.0 \mathrm{in}(2.54 \mathrm{~cm})$, and $2.0 \mathrm{in}(5.08 \mathrm{~cm})$, respectively. This arena layout was used for BILL-Ant-a testing described in Section 7.5.1 and Section 7.5.2.

\subsubsection{Irregular Terrain Navigation (No Goal Seeking)}

Testing was conducted with the robot tethered to a bench top power supply with a 10A limit for motor power, and another power supply with a $1 \mathrm{~A}$ limit for logic 
power. These power supplies were used since battery life was very limited and the charging time for all four batteries was approximately $8 \mathrm{hrs}$ (two batteries at a time, $4 \mathrm{hr}$ charge time).

Generally, BILL-Ant-a was able to navigate the random elevation test arena shown in Fig. 7.10. However, the performance was less than desirable.

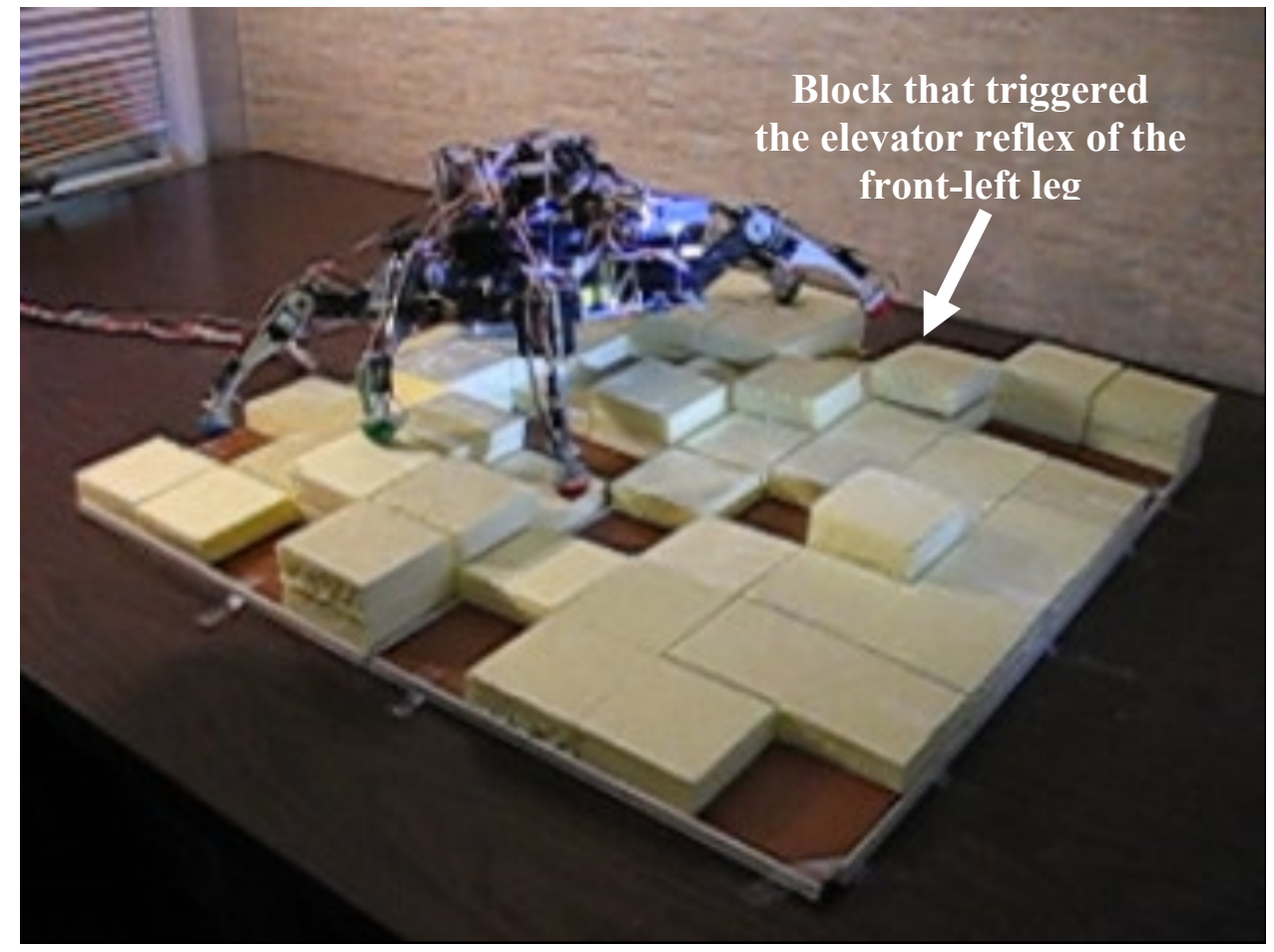

Figure 7.10. BILL-Ant-a in the random elevation field test arena.

The arena is configured with various block heights as determined by the custom software that chooses random elevation values for the arena grid. The arena layout shown here uses the block heights that are illustrated in Fig. 7.9. Currently, BILL-Ant-a is performing an elevator reflex with the left-front leg after it came in contact with a 2.0 in $(5.08 \mathrm{~cm})$ tall block.

Because of the motor power supply current limit, BILL-Ant-a suffered frequent over-current situations and powered down (at times during climbing, BILL-Ant-a demands up to about $14 \mathrm{~A}$ of current). Also, the limited current supply reduced 
the amount of torque that the $\mathrm{CTr}$ joint motor could exert, thereby reducing the climbing capabilities of the robot. A higher capacity power supply, or more available batteries, would have improved the results.

Still, with a few human interventions to reposition the robot when it became stuck due to a lack of available torque, BILL-Ant-a was able to navigate the arena during three of the five trials that were performed. The failed trials were each due to over-current situations shutting down the robot.

In part, the few successes were due to the blocks not being firmly secured to the arena substrate. As such, BILL-Ant-a kicked blocks out of the way as often as it climbed over them.

\subsubsection{Irregular Terrain Navigation with Goal Seeking}

As with the 'no goal seeking' scenarios, BILL-Ant-a had difficulty navigating the random elevation field arena due to motor current demand issues. The detection of the light-based goals, and directed turning efforts toward those goals, however, did perform without flaw.

Eight trials were conducted during this experiment, and the foam blocks were secured to the arena substrate with glue, in order to prevent the block kicking incidents seen in the previous trials. Since the blocks were no longer able to move out of the way, they presented greater resistance to the leg joint motors. This, in turn, caused more over-current situations, which led to the robot powering down more often. For the eight trials, only during two was BILL-Ant-a 
able to reach the other side of the arena, and those trials each required human intervention at least twice.

\subsubsection{BILL-Ant-a Performance Evaluation}

It was clear from the experiments performed with BILL-Ant-a that the reflex and goal seeking behaviors performed admirably. However, the limited amount of torque available to the $\mathrm{C} \operatorname{Tr}$ joint motors hindered the performance in the random elevation field arena.

It should be noted however, that all but one of the CTr joint motors on BILL-Anta are original to its predecessor hexapod and are six years old. Additionally, the joint motors performed all of the maximum load capacity experiments as part of the testing of BILL-Ant-p and now likely have reduced maximum torque capabilities.

The availability of a higher current power source would have resulted in better success in navigating the arena to a small extent, but realistically, more powerful CTr joint motors would be required to improve the performance of BILL-Ant-a. 


\title{
CHAPTER VIII
}

\author{
FUTURE WORK
}

\subsection{General}

While this is the end of the BILL-Ant series of projects, there are several updates and improvements that would enhance the performance of the hexapod.

\subsection{Faster, More Capable Microcontrollers}

The BrainStem microcontrollers were intentionally chosen for their low computation capabilities in order to demonstrate the computational simplicity of the SCASM method of intra-leg joint control to generate stepping motions, and the BILL-LEGS method of inter-leg gait coordination. However, these microcontrollers were barely sufficient for the task and only produced the most basic of walking, reacting, and goal-seeking behaviors.

A network of faster, more capable microcontrollers would have faster control cycles that would add a much-improved level of smoothness to the walking motions by being able to implement higher-resolution joint movements, more responsive joint state transitions, and active compliance. The improved effects of a faster processor can be seen in the implementation of the Single-Leg Test Platform, whose control cycle is nearly $200 \mathrm{~Hz}$; well above the $5 \mathrm{~Hz}$ speed of BILL-Ant-a. The Single-Leg Test Platform exhibits very smooth stepping, has 
active compliance, and produces more repeatable stepping motion patterns than BILL-Ant-a.

\subsection{Upgraded, More Powerful CTr Joint Motors}

As was seen with the limited successes of BILL-Ant-a to navigate the random elevation test arena, new $\mathrm{C} \operatorname{Tr}$ joint motors are required. The existing servo motors should be up to the task, however, years of use and the rigorous testing of the earlier hexapod, BILL-Ant-p, have left their torque capacities reduced. Rather than replace the servos with similar motors, it would be a great improvement to upgrade the joint actuators to newer, high-torque digital servos.

Currently available digital servo motors, such as the Hitec HSR-5990TG have physical characteristics similar to standard hobby servos (outer dimensions, mounting tab locations, etc.). Differences however, can be found inside the motor housing.

Digital driver circuits have higher motor $\mathrm{H}$-bridge control frequencies $(50 \mathrm{~Hz}$ as compared to some analog servos with only $10 \mathrm{~Hz}$ speeds) that apply motor actuation voltages at a higher rate. This higher rate equates to more available motor torque (although at a cost of a higher operating current), which improves the motive capabilities of the servo.

The Hitec HSR-5990TG has a stall torque of $333.29 \mathrm{oz}-\mathrm{in}(2.35 \mathrm{Nm})$ at $6.0 \mathrm{vdc}$, which is much higher than the MPI MX-450HP whose maximum torque is only $116.0 \mathrm{oz}$-in $(0.82 \mathrm{Nm})$ at the same voltage. Additionally, the Hitec servo can be 
operated at up to $7.4 \mathrm{vdc}$, which would allow the motors to be directly powered by Li-ion and Li-poly batteries without the need for voltage regulators. At this higher voltage, the HSR-5990TG servo can produce up to $416.61 \mathrm{oz}-\mathrm{in}(2.94 \mathrm{Nm})$ of torque (Tower Hobbies 2007).

\subsection{Newer Style Robotic Servo Motors with Bi-Directional Data Flow}

Another option to the Hitec HSR-5990TG servo motor upgrade, mentioned in the previous section, would be the use of the newer style robotic servo motors that allow bi-directional data flow. Information such as commanded motor position, PID control settings, motor speed, and maximum allowable torque (up to the physical maximum for the motor) can be sent to the motor from the host controller. Data such as actual motor position, current (motor load), and temperature can be retrieved from the motor and used for closed-loop control methods.

Motors such as the Dynamixel series from Robotis (Robotis, Seoul, Korea), the Kondo KRS series of servos that are part of the KHR humanoid robot kits (Kondo Kagaku Co., Ltd., Tokyo, Japan), and the Hitec servo motors used with the Robonova humanoid kit are all examples of motors with bi-directional communication capabilities. While these motors require different control methods, such as an RS-485 communication protocol instead of a pulse width command, the update in control hardware is worth the available data feedback from the motors themselves. 
Each of this type of servo motor is physically different from one another and also differs from standard hobby servos, and so would require an extensive redesign of the BILL-Ant-a hexapod. An alternative would be the OpenServo project control board (OpenServo 2008). This item is a servo controller that replaces the small board located inside the servo motor enclosure. In doing so, the pulse width position control is replaced by an $\mathrm{I}^{2} \mathrm{C}$-style two-wire communication method that allows a host system to send position commands, set PID control parameters, define motor speed, etc., while also being able to retrieve data such as current motor position, current (load) information, and motor speed. This is similar to the capabilities offered by the robotic servos mentioned above, however, a benefit to this option is that standard hobby servos can be retrofitted with this control board such that no physical redesign of the hexapod is required.

\subsection{New, Biologically-based Physical Design}

The previous sections in this chapter present options that would improve BILLAnt-a in its current physical state. However, another limitation found during the experiments with the robot was its physical design, in regard to the placement and geometry of the legs. The closely-positioned ThC joints limited the available protraction/retraction ranges-of-motion for the legs, and the joint motors with their \pm 45 deg movement arcs is greatly reduced from that of actual insects, which have joint movement capabilities of up to 150deg (Cruse 1976).

An updated design with to-scale thorax and leg segment lengths, ThC joint mounting positions and angles relative to the thorax, and joint ranges-of-motion at 
or near $180 \mathrm{deg}$ would be a vast improvement over the current design. Such design changes provide the legs with more movement freedom and fewer control restrictions as a result.

8.5 Further Research into the Addition of More Sensory Systems and Descending Modulation

Since the infrastructure has been created by this work such that high-level systems can modulate the low-level leg controllers, it is now possible to add more sensory systems and a more complex "brain". These additions would allow the robot to possess much richer and more complex behaviors than have been implemented so far. These more complex behaviors would add greater functionality to the robot, enabling it to perform a larger variety of tasks and navigate a more diverse environment, complete with different obstacles and a variety of goals. 


\section{LIST OF REFERENCES}

(LISTED ALPHABETICALLY BY FIRST AUTHOR, THEN IN REVERSE CHRONOLOGICAL ORDER)

A. Abbott. (2007) "Working Out the Bugs," in Nature, Vol. 445, pp. 250-253, January $18,2007$.

E. Ackerman. (2010) "Updated A-Pod Is Faster, More Nimble," World Wide Web, http://www.botjunkie.com/2010/04/21/updated-a-pod-is-faster-more-nimble/, April 21, 2010.

T. Akay, U. Bässler, P. Gerharz, and A. Büschges. (2001) “The Role of Sensory Signals from the Insect Coxa-Trochanteral Joint in Controlling Motor Activity of the Femur-Tibia Joint," in Journal of Neurophysiology, vol. 85, pp. 594-604, 2001.

T. Akay, S. Haehn, J. Schmitz, and A. Büschges. (2004) "Signals from Load Sensors Underlie Interjoint Coordination During Stepping Movements of the Stick Insect Leg” in Journal of Neurophysiology, vol. 92, pp. 42-51, 2004.

T. J. Allen, R. D. Quinn, R. J. Bachmann, and R. E. Ritzmann. (2003) "Abstracted Biological Principles Applied with Reduced Actuation Improve Mobility of Legged Vehicles," in Proceedings of the IEEE/RSJ International Conference on Intelligent Robots and Systems (IROS'03): Las Vegas (NV), USA, October 28-30, 2003.

C. Angle. (1991) Design of an Artificial Creature, S. M. Thesis, Department of Electrical Engineering and Computer Science, MIT, Cambridge, MA, USA, June 1991.

S. A. Bailey, J. G. Cham, M. R. Cutcosky, and R. J. Full. (2000) "Comparing the Locomotion Dynamics of the Cockroach and a Shape Deposition Manufactured Biomimetic Hexapod," 2000.

D. P. Barnes. (1998) "Hexapodal Robot Locomotion over Uneven Terrain," in Proc. IEEE Conf. on Control Applications. Trieste, Italy, pp. 441-445, September 1998.

U. Bässler and A. Büschges. (1998) "Pattern Generation for Stick Insect Walking Movements - Multisensory Control of a Locomotor Program," in Brain Research Reviews, vol. 27, pp. 65-88, 1998.

U. Bässler. (1993) “The Femur-Tibia Control System of Stick Insects - A Model System for the Study of the Neural Basis of Joint Control," in Brain Research Reviews, vol. 18, pp. 207-226, 1993. 
U. Bässler, J. Rohrbacher, G. Karg, and G. Breutel. (1991) "Interruption of Searching Movements of Partially Restrained Front Legs of Stick Insects, A Model Situation for the Start of Stance Phase?" in Biological Cybernetics, vol. 65, pp. 507-514, 1991.

R. D. Beer, R. D. Quinn, H. J. Chiel, and R. E. Ritzmann. (1997) "Biologically Inspired Approached to Robotics: What Can We Learn from Insects?" in Communications of the ACM, vol. 40, no. 3, pp. 30-38, March 1997.

W. Bell. (1990) "Searching Behavior Patterns in Insects," in Annu. Rev. Entomol. 1990. $35: 447-67$.

K. Berns. (2005) "The Walking Machine Catalog: Walking Machine Catalog," World Wide Web, http://www.walking-machines.org/, 2005.

M. J. Boggess, R. T. Schroer, R. D. Quinn, and R. E. Ritzmann. (2004) "Mechanized Cockroach Footpaths Enable Cockroach-like Mobility," in Proc. IEEE Conf. on Robotics and Automation (ICRA'04), vol. 3, pp. 2871-2876, New Orleans, LA, USA, April 26-May 1, 2004.

R. A. Brooks. (1989) "A Robot That Walks; Emergent Behaviors from a Carefully Evolved Network" Technical Report, MIT AI Lab Memo 1091, Cambridge, MA, USA February 1989.

D. Bucher, T. Akay, R. A. DiCaprio, and A. Büschges. (2003) "Interjoint Coordination in the Stick Insect Leg-Control System: The Role of Positional Signaling," in Journal of Neurophysiology, vol. 89, pp. 1245-1255, 2003.

Buggin' with Ruud, hosted by Ruud Kleinpaste, Episode 9, "Bug Super Heroes," Discovery Channel, Animal Planet, 2006.

A. Büschges. (2005) "Sensory Control and Organization of Neural Networks Mediating Coordination of Multisegmental Organs for Locomotion," in Journal of Neurophysiology, vol. 93, pp. 1127-1135, 2005.

A. Büschges, B. C. Ludwar, D. Bucher, J. Schmidt, and R. A. DiCaprio. (2004) "Synaptic Drive Contributing to Rhythmic Activation of Motoneurons in the Deafferented Stick Insect Walking Systems," in European Journal of Neuroscience, vol. 19, pp. 1856-1862, 2004.

A. Büschges, J. Schmitz, and U Bässler. (1995) "Rhythmic Patterns in the Thoracic Nerve Cord of the Stick Insect Induced by Pilocarpine," in Journal of Experimental Biology, vol. 198, pp. 453-456, 1995.

A. Buschmann. (2000) "Home of Tarry I \& II: Frequently Asked Questions about Tarry," World Wide Web, http://www.tarry.de, March 2000. (2000b) 
A. Buschmann. (2000) "Home of Tarry I \& II: Design of the Walking Machine Tarry II," World Wide Web, http://www.tarry.de, March 2000. (2000a)

"Creature Features," in the Case Engineering Magazine, Case Western Reserve University, Vol. 4, Issue 1, pp. 16-19, Fall 2005.

H. Cruse, V. Dürr, and J. Schmitz. (2007) "Insect Walking is Based on a Decentralized Architecture Revealing a Simple and Robust Controller," in Philosophical Transactions of the Royal Society A, vol. 365, pp. 221-250, 2007

H. Cruse, V. Dürr, J. Schmitz, and A. Schneider. (2003) "Control of Hexapod Walking in Biological Systems," in Proceedings of the $2^{\text {nd }}$ International Symposium on Adaptive Motion of Animals and Machines (AMAM'03), Kyoto, Japan, March 48, 2003.

H. Cruse, U. Müller-Wilm, J. Dean. (1993) "Artificial Neural Nets for Controlling a 6legged Walking System," in Proc. of the Second International Conference on Simulation of Adaptive Behavior: From Animals to Animats 2, MIT Press, Cambridge, MA, pp. 52-60, 1993.

H. Cruse. (1990) "What Mechanisms Coordinate Leg Movement in Walking Arthropods?" Trends in Neurosciences, vol. 13, pp. 15-21, 1990.

H. Cruse and U. Müller. (1986) "Two Coupling Mechanisms which Determine the Coordination of Ipsilateral Legs in the Walking Crayfish," J. exp. Biol., vol. 121, pp. 349-369, 1986.

H. Cruse. (1985) "Coactivating Influences between Neighbouring Legs in Walking Insects," J. exp. Biol., vol. 114, pp. 513-519, 1985.

H. Cruse and J. Storrer. (1977) "Open Loop Analysis of a Feedback Mechanism Controlling the Leg Position in the Stick Insect Carausius morosus: Comparison between Experiment and Simulation," Biol. Cybern., vol. 25, pp. 143 - 153, 1977.

H. Cruse. (1976) "On the Function of the Legs in the Free Walking Stick Insect Carausius morosus," in J. Comp. Physiol., vol. 112, pp. 235-262, 1976.

G. S. Cymbalyuk, R. M. Borisyuk, U. Müller-Wilm, and H. Cruse. (1998) "Oscillatory Networks Controlling Six-Legged Locomotion," in Neural Networks, 11, 14491460, 1998.

J. Dean. (1991) "Effect of Load on Leg Movement and Step Coordination of the Stick Insect Carausius Morosus," in J. exp. Biol., vol. 159, pp. 449-471, 1991. (1991c) 
J. Dean. (1991) "A Model of Leg Coordination in the Stick Insect, Carausius morosus; II. Description of the kinematic model and simulation of normal step patterns," in Biological Cybernetics, vol. 64, pp. 403-411, 1991. (1991b)

J. Dean. (1991) “A Model of Leg Coordination in the Stick Insect, Carausius morosus; I. Geometrical consideration of contralateral and ipsilateral coordination mechanisms between two adjacent legs," in Biological Cybernetics, vol. 64, pp. 393-402, 1991. (1991a)

J. Dean and G. Wendler. (1984) "Stick Insect Locomotion on a Wheel: Patterns of Stopping and Starting," in J. exp. Biol., 110, 203-216, 1984.

M. Denton. (2008) "Micromagic Systems," World Wide Web, http://www.micromagicsystems.com/, 9 June 2008.

P. A. Dunker, W. A. Lewinger, A. J. Hunt, and R. D. Quinn. (2009) “An Insect-Inspired Robot for Lunar In-Situ Resource Utilization," in Proceedings of the IEEE/RSJ International Conference on Intelligent Robots and Systems (IROS'09): St. Louis (MO), USA, October 11-15, 2009.

P. A. Dunker, W. A. Lewinger, R. D. Quinn, J. J. Caruso, and M. J. Krasowski. (2008) "Biologically Inspired Robot for Lunar Soil Excavation," Adaptive Motion of Animals and Machines, Cleveland (OH), USA, June 1-6, 2008. (2008b)

P. A. Dunker, W. A. Lewinger, R. D. Quinn, J. J. Caruso, M. J. Krasowski. (2008) "Biologically Inspired Robot for Lunar Soil Excavation," Research ShowCASE, Case Western Reserve University, Cleveland (OH), USA, April 16-17, 2008. (2008a)

P. A. Dunker, W. A. Lewinger, R. D. Quinn, J. J. Caruso, and M. J. Krasowski. (2007) "Biologically Inspired Robot for Lunar Soil Excavation," Research ShowCASE, Case Western Reserve University, Cleveland (OH), USA, April 11-12, 2007. (Awarded First Place)

V. Dürr and W. Ebeling. (2005) "The Behavioural Transition from Straight to Curve Walking: Kinetics of Leg Movement Parameters and the Initiation of Turning," in The Journal of Experimental Biology, vol. 208, pp. 2237-2252, 2005.

Ö. Ekeberg, M. Blümel, and A Büschges. (2004) "Dynamic Simulation of Insect Walking," in Arthropod Structure \& Development, vol. 33, pp. 287-300, 2004.

K. S. Espenschied, R. D. Quinn, H. J. Chiel, and R. D. Beer. (1996) "Biologically Based Distributed Control and Local Reflexes Improve Rough Terrain Locomotion in a Hexapod Robot," in Robotics and Autonomous Systems, vol. 18, pp. 59-64, 1996. 
K. S. Espenschied, R. D. Quinn, H. J. Chiel, and R. D. Beer. (1994) "BiologicallyInspired Hexapod Robot Control," in Proc. $5^{\text {th }}$ International Symposium on Robotics and Manufacturing: Research, Education and Applications (ISRAM '94), vol. 5, pp. 89-94, Maui, Hawaii, August 14-18, 1994.

K. S. Espenschied and R. D. Quinn. (1994) "Biologically-Inspired Hexapod Robot Design and Simulation," in Proc. AIAA Conference on Intelligent Robots in Field, Factory, Service and Space, Houston, Texas, March 20-24, 1994.

K. S. Espenschied, R. D. Quinn, H. J. Chiel, and R. D. Beer. (1993) "Leg Coordination Mechanisms in Stick Insect Applied to Hexapod Robot Locomotion," in Adaptive Behavior, vol. 1, no. 4, pp. 455-468, 1993.

C. Ferrell. (1993) "Robust Agent Control of an Autonomous Robot with Many Sensors and Actuators," Technical Report, MIT Artificial Intelligence Lab, TR-1443, Cambridge, MA, USA, 1993.

H. Fischer, J. Schmidt, and A. Büschges. (2001) "Pattern Generation for Walking and Searching Movements of a Stick Insect Leg I. Coordination of Motor Activity," in Journal of Neurophysiology, vol. 85, pp. 341-353, 2001.

M. Frik, M. Guddat, M. Karatas, and C.D. Losch. (1999) "A Novel Approach to Autonomous Control of Walking Machines," in Proceedings of the 2nd International Conference on Climbing and Walking Robots (CLAWAR'99), pp 333-342, Portsmouth, UK, Sept. 13-15, 1999.

B. Gaßmann, K.-U. Scholl, and K. Berns. (2001) "Behavior Control of LAURON III for Walking in Unstructured Terrain," in Proc. Intl. Conference on Climbing and Walking Robots (CLAWAR'01), pp. 651-658, Karlsruhe, Germany, September 2001.

Graduate Student News, "Case Bio-Robots Keep Moving Forward," in the Electrical Engineering and Computer Science Department Digest, Case Western Reserve University, p. 8, Spring 2006.

D. Graham. (1972) "A Behavioural Analysis of the Temporal Organisation of Walking Movements in the $1^{\text {st }}$ Instar and Adult Stick Insect," in J. comp. Physiol., 18, 23$52,1972$.

C. M. Harley, W. A. Lewinger, R. E. Ritzmann, R. D. Quinn. (2005) "Characterization of Obstacle Avoidance Behaviors in the Cockroach Blaberus discoidalis and Implementation in a Semi-Autonomous Robot," Program No. 176.10. Washington, DC: Society for Neuroscience, 2005.

C. M. Harley, W. A. Lewinger, J. Talley. (2004) "Examining Cockroach Climbing and Tunneling Behaviors in Response to Visual and Tactile Cues and Mimicking the 
Tactile Behavior using a Biologically-Inspired Mobile Robot with Mechanical Antennae", Neuromechanics Fellowship Project for NSF IGERT Grant, Summer 2004.

D. Hess and A. Büschges. (1999) "Role of Proprioceptive Signals from an Insect FemurTibia Joint in Patterning Motoneuronal Activity of an Adjacent Leg," in Journal of Neurophysiology, vol. 81, pp. 1856-1865, 1999.

D. Hess and A. Büschges. (1997) "Sensorimotor Pathways Involved in Interjoint Reflex Action of an Insect Leg," in Journal of Neurobiology, vol. 33, issue 7, pp. 891913, 1997.

T. Hoffmann and U. Bässler. (1982) "Anatomy and Physiology of Trochanteral Campaniform Sensilla in the Stick Insect, Culiculina impigra," in Physiological Entomology, vol. 7, pp. 413-426, 1982.

B. Hölldobler and E. Wilson. (1990) The Ants, The Belknap Press of Harvard University Press, Cambridge, Massachusetts, 1990.

S. L. Hooper, C. Guschlbauer, M. Blümel, P. Rosenbaum, M. Gruhn, T. Akay, A. Büschges. (2009) "Neural Control of Unloaded Leg Posture and of Leg Swing in Stick Insect, Cockroach, and Mouse Differs from That in Larger Animals," in $J$. Neurosci., vol. 29, pp. 4109-4119, 2009.

ICRA 2005 Award Winners: ICRA Best Video Proceedings - "Insect-like Antennal Sensing for Climbing and Tunneling Behavior in a Biologically-inspired Mobile Robot," William A. Lewinger, Cynthia M. Harley, Roy E. Ritzmann, Michael S. Branicky, and Roger D. Quinn (Case Western Reserve University, USA), IEEE Robotics and Automation, Society News, Vol. 12, No. 2, p. 8, September 2005.

John Lydon's Megabug, hosted by John Lydon, Ep. 7, "Cockroaches," Discovery Channel, 2004.

Kickin' It with Kenny, hosted by Kenny Crumpton, FOX8 News, Cleveland OH, April 12, 2007.

Kickin' It with Kenny, hosted by Kenny Crumpton, FOX8 News, Cleveland OH, April 6, 2006.

T. Kindermann, H. Cruse, and J. Dean. (1998) "A Biologically Motivated Controller for a Six-Legged Walking System," in Proc. of the 24th Annual Conference of the IEEE Industrial Electronics Society (IECON 98), vol. 4, pp. 2168-2173, Aachen, Germany, Sept. 1998.

LERN, "Research Bugs,” in Robot Magazine, Issue 8, p. 20, Fall 2007. 
W. A. Lewinger. (2011) "Insect-Inspired, Actively Compliant Robotic Hexapod," Ph.D. Dissertation, Department of Electrical Engineering and Computer Science, Systems and Control Engineering, Case Western Reserve University, Cleveland, OH, USA. May 2011. (this document)

W. A. Lewinger and R. D. Quinn. (2011) "Neurobiologically-Based Control System for An Adaptively Walking Hexapod," in Industrial Robot, IR 38-3, 2011. (awaiting printing) (invited publication)

W. A. Lewinger and R. D. Quinn. (2010) "A Hexapod Walks Over Irregular Terrain Using a Controller Adapted from an Insect's Nervous System," in Proceedings of the IEEE/RSJ International Conference on Intelligent Robots and Systems (IROS'10): Taipei, Taiwan, October 18-22, 2010. (2010b)

W. A. Lewinger and R. D. Quinn. (2010) "Neurobiologically-Based Control System for An Adaptively Walking Hexapod," in Proceedings of the International Conference on Climbing and Walking Robots (CLAWAR'10), Nagoya, Japan, Aug. 31- Sept. 03, 2010. (2010a) (Won "Innovation Award 2010 - For Practical Innovation in the Field of Robotics")

W. A. Lewinger and R. D. Quinn. (2009) "A Small, Autonomous, Agile Robot with an On-board, Neurobiologically-based Control System," in Video Proceedings of the IEEE/RSJ International Conference on Intelligent Robots and Systems (IROS'09): St. Louis (MO), USA, October 11-15, 2009.

W. A. Lewinger, C. M. Harley, M. S. Watson, M. S. Branicky, R. E. Ritzmann, and R. D. Quinn. (2009) "Animal-Inspired Sensing for Autonomously Climbing or Avoiding Obstacles," in Applied Bionics and Biomechanics, Vol. 6, Issue 1, pp. 3-21, 2009.

W. A. Lewinger, B. L. Rutter, R. D. Quinn. (2008) "Irregular Terrain Navigation and Leg Coordination Improve Walking Behavior for Small Legged Robots." Program No. 198.8. Washington, DC: Society for Neuroscience, 2008.

W. A. Lewinger, P. A. Dunker, and R. D. Quinn. (2008) "Proof of Concept for a Small Lunar Excavation Robot," Abstract for Planetary and Terrestrial Mining Sciences Symposium (PTMSS'08), Montréal (QC), Canada, June 8-11, 2008.

W. A. Lewinger and R. D. Quinn. (2008) "BILL-LEGS: Low computation Emergent Gait System for Small Mobile Robots," in the Proceedings of the IEEE International Conference on Robotics and Automation (ICRA'08): pp. 251-256, Pasadena (CA), USA, May 11-23, 2008.

W. A. Lewinger, P. A. Dunker, and R. D. Quinn. (2007) "Lunar Excavation Robots: Size Matters," Abstract for Planetary and Terrestrial Mining Sciences Symposium (PTMSS'07), Sudbury (ON), Canada, June 10-13, 2007. 
W. A. Lewinger (2006) "Rapid and Robust Identification of Common Road Markings and Traffic Signals in Urban Environments to Generate Control Signals for Autonomous Vehicles", Case Western Reserve University, EECS 490, Digital Image Processing, Final Project, December 2006. (2006b)

W. A. Lewinger (2006) "Using Color Separation to Quickly and Robustly Identify Traffic Markings in Urban Environments", Case Western Reserve University, EECS 490, Digital Image Processing, Mid-Term Project, November 2006. (2006a)

W. A. Lewinger, M. S. Watson, and R. D. Quinn. (2006) "Obstacle Avoidance Behavior for a Biologically-Inspired Mobile Robot Using Binaural Ultrasonic Sensors," in Video Proceedings of the IEEE/RSJ International Conference on Intelligent Robots and Systems (IROS'06), Beijing, China, October 9-13, 2006. (2006b)

W. A. Lewinger, M. S. Watson, and R. D. Quinn. (2006) "Obstacle Avoidance Behavior for a Biologically-Inspired Mobile Robot Using Binaural Ultrasonic Sensors," in Proceedings of the IEEE/RSJ International Conference on Intelligent Robots and Systems (IROS'06): pp. 5769-5774, Beijing, China, October 9-13, 2006. (2006a)

W. A. Lewinger, B. L. Rutter, M. Blümel, A. Büschges, and R. D. Quinn. (2006) "Sensory Coupled Action Switching Modules generate robust, adaptive stepping in legged robots," in Proceedings of the International Conference on Climbing and Walking Robots (CLAWAR'06), Brussels, Belgium, Sept. 12-14, 2006.

W. A. Lewinger, C. M. Harley, M. S. Watson, R. E. Ritzmann, M. S. Branicky, R. D. Quinn. (2006) "Borrowing from Nature: Biologically-Inspired Robotics," Research ShowCASE, Case Western Reserve University, Cleveland (OH), USA, April 5-6, 2006.

W. A. Lewinger, M. S. Branicky, and R. D. Quinn. (2005) "Insect-Inspired, Actively Compliant Robotic Hexapod," in Proceedings of the International Conference on Climbing and Walking Robots (CLAWAR'05): pp. 65-72, London, U.K., Sept. 13-15, 2005.

W. A. Lewinger. (2005) "Insect-Inspired, Actively Compliant Robotic Hexapod," M.S. Thesis, Department of Electrical Engineering and Computer Science, Case Western Reserve University, Cleveland, OH, USA. May 2005.

W. A. Lewinger, C. M. Harley, R. E. Ritzmann, M. S. Branicky, and R. D. Quinn. (2005) "Insect-like Antennal Sensing for Climbing and Tunneling Behavior in a Biologically-inspired Mobile Robot," in Proceedings of the IEEE International Conference on Robotics and Automation (ICRA'05): pp. 4187-4192, Barcelona, Spain, April 18-22, 2005. (2005a) 
W. A. Lewinger, C. M. Harley, R. E. Ritzmann, M. S. Branicky, and R. D. Quinn. (2005) "Insect-like Antennal Sensing for Climbing and Tunneling Behavior in a Biologically-inspired Mobile Robot," in Proceedings of the IEEE International Conference on Robotics and Automation (ICRA'05): pp. 4187-4192, Barcelona, Spain, April 18-22, 2005. (2005b) (Won Best Video Award)

"Like an Animal," in the IEEE Electronic Systems and Software Magazine, Vol. 3, Issue 6, pp. 10-14, December/January 2005/2006.

Lynxmotion BH3. (2008) "BH3," World Wide Web, http://www.lynxmotion.com/Category.aspx?CategoryID=33, 2008.

Lynxmotion Phoenix. (2008) "Phoenix," World Wide Web, http://www.lynxmotion.com/Category.aspx?CategoryID=117, 2008.

Lynxmotion Servo Erector Set. (2008) "Servo Erector Set," World Wide Web, http://www.lynxmotion.com/Category.aspx?CategoryID=73, 2008.

P. Manoonpong, F. Pasemann, and F. Wörgötter. (2008) "Sensor-Driven Neural Control for Omnidirectional Locomotion and Versatile Reactive Behaviors of Walking Machines," in Robotics and Autonomous Systems, Volume 56, Issue 3 (March 2008), Pages 265-288, 2008.

Material World, hosted by Quentin Cooper, BBC Radio 4, April 16, 2009.

R. B. McGhee and G. I. Iswandhi. (1979) "Adaptive Locomotion of a Multilegged Robot over Rough Terrain," in IEEE Transactions on Systems, Man, and Cybernetics (SMC-9), (4), 176-182, 1979.

New Micros, Inc. (2003) IsoPod ${ }^{\mathrm{TM}}$ Users Manual: Virtually Parallel Machine Architecture, Dallas, TX, USA, 2003.

J. M. Morrey, B. Lambrecht, A. D. Horchler, R. E. Ritzmann, and R. D. Quinn. (2003) "Highly Mobile and Robust Small Quadruped Robots," in Proceedings of the IEEE/RSJ International Conference on Intelligent Robots and Systems (IROS'03): Las Vegas (NV), USA, October 28-30, 2003.

L. Mu and R.E. Ritzmann. (2005) "Kinematics and Motor Activity During Tethered Walking and Turning in the Cockroach, Blaberus discoidalis," in J. Comp. Physiol A., 191: 1037-1054, 2005.

Nature Tech - How Engineers are Inspired by Nature's Top Design, "The Magic of Motion", Written/Produced by Steve Nicholls and Alfred Vendl, ORF Universum, Vienna, Austria, 2006. 
OpenServo. (2008) “OpenServo High Quality Open Source Digital Servo,” World Wide Web, http://www.openservo.com/, 2008.

F. Pfeiffer, H. J. Weidemann, and J. Eltze. (1994) "The TUM Walking Machine. - In: Intelligent Automation and Soft Computing," in Trends in Research, Development and Applications, TSI Press, vol. 2, pp. 167-174, 1994.

N. Porcino. (1990) "Hexapod Gait Control by a Neural Network," in Proceedings of the International Joint Conference on Neural Networks, San Diego (CA), USA, vol. 1, pp. 189-194, 1990.

R. D. Quinn, R. E. Ritzmann, A. D. Horchler, W. A. Lewinger, R. J. Bachmann, A. S. Boxerbaum. (2007) "Improving Robot Locomotion and Autonomy through Inspiration From Animal Neuromechanical Designs," Research ShowCASE, Case Western Reserve University, Cleveland (OH), USA, April 11-12, 2007.

R. D. Quinn, R. E. Ritzmann, A. D. Horchler, W. A. Lewinger, R. J. Bachmann, A. S. Boxerbaum. (2006) "Improving Robot Locomotion and Autonomy through Inspiration From Animal Neuromechanical Designs," Research ShowCASE, Case Western Reserve University, Cleveland (OH), USA, April 5-6, 2006.

M. Raibert, K. Blankespoor, G. Nelson, and R. Playter. (2008) "Big Dog, The RoughTerrain Quadruped Robot," Prodeedings of the 17 $17^{\text {th }}$ World Congress The International Federation of Automatic Control, Seoul Korea, July 6-11, 2008.

E. A. Rietman and R. W. Hillis. (2006) "Neural Computation with Rings of Quasiperiodic Oscillators," Physical Sciences, Inc., Report PSI SR-1278, arXiv.org (27 November 2006).

B. L. Rutter, W. A. Lewinger, M. Blümel, A. Büschges, and R. D. Quinn. (2007) “Simple Muscle Models Regularize Motion in a Robotic Leg with Neurally-Based Step Generation," in Proceedings of the IEEE Intl. Conference on Intelligent Robots and Systems (ICRA'07), Roma, Italy, April 10-14, 2007.

B. L. Rutter, W. A. Lewinger, B. K. Taylor, M. Wilson, M. Blümel, Ö. Ekeberg, A. Büschges, R. E. Ritzmann, R. D. Quinn. (2008) "Neurally-Based Robot Control for Neuromechanical Modeling of Insect Stepping," Adaptive Motion of Animals and Machines, Cleveland (OH), USA, June 1-6, 2008. (2008b)

B. L. Rutter, W. A. Lewinger, B. K. Taylor, M. Wilson, M. Blümel, Ö. Ekeberg, A. Büschges, R. E. Ritzmann, R. D. Quinn. (2008) "Neurally-Based Robot Control for Neuromechanical Modeling of Insect Stepping," Research ShowCASE, Case Western Reserve University, Cleveland (OH), USA, April 16-17, 2008. (2008a) (Awarded Honorable Mention) 
B. L. Rutter, W. A. Lewinger, B. K. Taylor, M. Wilson, M. Blümel, Ö. Ekeberg, A. Büschges, R. E. Ritzmann, R. D. Quinn. (2007) "Neurally-Based Robot Control for Neuromechanical Modeling of Insect Stepping," Research ShowCASE, Case Western Reserve University, Cleveland (OH), USA, April 11-12, 2007. (2007b) (Awarded Honorable Mention)

B. L. Rutter, W. A. Lewinger, B. K. Taylor, M. Wilson, M. Blümel, Ö. Ekeberg, A. Büschges, R. E. Ritzmann, R. D. Quinn. (2007) Neurally-Based Robot Control for Neuromechanical Modeling of Insect Stepping. Program No. 449.13. Washington, DC: Society for Neuroscience, 2007. (2007a)

U. Saranli, M. Buehler and D. Koditschek. (2001) "RHex a Simple and Highly Mobile Hexapod Robot." Int. J. Robotics Research, 20(7): 616-631, 2001.

J. Schmitz, J. Dean, T. Kindermann, M. Schumm, and H. Cruse. (2001) “A Biologically Inspired Controller for Hexapod Walking: Simple Solutions by Exploiting Physical Properties," in Biol. Bull., 200:195-200, April 2001.

J. Schmitz. (1998) "Biological Cybernetics / Theoretical Biology: Leg Coordination," World Wide Web, http://www.unibielefeld.de/biologie/Kybernetik/research/walk.html, December 7, 1998.

Six-Legged Soldiers: Using Insects as Weapons of War, Jeffrey A. Lockwood, Oxford University Press, pp. xvi and 293, 2009.

Tower Hobbies. (2007) "Hitec HSR-5990TG Digital HMI Ultra Premium Robot Servo," World Wide Web, http://www3.towerhobbies.com/cgibin/wti0001p?\&I=LXRRC5\&P= 7, 2007.

J. T. Watson and R. E. Ritzmann. (1998) "Leg Kinematics and Muscle Activity during Treadmill Running in the Cockroach, Blaberus discoidalis: I. Slow Running," in J. Comp. Physiol., Vol. 182: 11-22, 1998.

T. E. Wei, R. D. Quinn, and R. E. Ritzmann, "A CLAWAR That Benefits from Abstracted Cockroach Locomotion Principles" in Proceedings of the International Conference on Climbing and Walking Robots (CLAWAR'04), Madrid, Spain, Sept. 22-24, 2004.

H.-J. Weidermann, F. Pfeiffer, J. Eltze. (1994) "The Six-Legged TUM Walking Robot," in Proceedings of IEEE/RSJ/GI International Conference of Intelligent Robots and Systems (IROS'94), vol. 2, pp. 1026-1033, Munich Germany, Sept. 12-16, 1994.

J. Wessnitzer and B. Webb. (2006) "Multimodal Sensory Integration in Insects Towards Insect Brain Control Architectures," in Bioinspiration and Biomimetics, Vol. 1, pp. 62-75, 2006. 
D. M. Wilson. (1966) "Insect Walking," in Annual Review of Entomology, vol. 11, pp. 103-122, 1966.

Xan. (2009) "Lynxmotion Project Page - BlackWido," Jeroen Janssen (a.k.a. Xan), World Wide Web, http://www.lynxmotion.com/images/html/proj102.htm, 2009.

H. Yahya. (2000) The Miracle in the Ant, Ta-Ha Publishers, Inc., London, United Kingdom, 2000.

V. Yodaiken and M. Barabanov. (1997) A Real-Time Linux, Linux Applications Development and Deployment Conference (USELINUX), 1997.

Zenta. (2009) “A-pod: A Robot Ant's Tale « Trossen Robotics Blog,” Kåre Halvorsen (a.k.a. Zenta), World Wide Web, http://blog.trossenrobotics.com/2009/03/17/apod-a-robot-ants-tale/, March 17, 2009. (2009b)

Zenta. (2009) "[Project] A-pod, An Ant Inspired Hexapod - Trossen Robotics Community," Kåre Halvorsen (a.k.a. Zenta), World Wide Web, http://forums.trossenrobotics.com/showthread.php?t=2762, January 6, 2009. (2009a) 